Michael Ganske

\title{
Intertemporale Aspekte von Staatsverschuldung und Außenhandel
}


Michael Ganske

\section{Intertemporale Aspekte von Staatsverschuldung und Außenhandel}

Staatsverschuldung und Außenhandel wirken über verschiedene Kanäle auf die Entwicklung von Volkswirtschaften. Um die Wirkungsmechanismen zu eruieren, werden mikroökonomisch fundierte dynamische makroökonomische Modelle diskutiert.Zur Anwendung kommen Modelle überlappender Generationen. In ihnen besitzt zwar die modellierte Ökonomie einen unendlichen Zeithorizont, nicht aber die sie bevölkernden Akteure. Untersucht werden sowohl Allokationswirkungen als auch Zins- und Wohlfahrtseffekte. Es zeigt sich, daß die Aufnahme von Außenhandel und die Finanzierungsentscheidung des Staates entscheidend auf die Entwicklung der modellierten Ökonomien wirkt. Bei Freihandel besitzt der staatliche Verschuldungsprozeß, zusätzlich $\mathrm{zu}$ den intergenerationalen Umverteilungswirkungen, internationale Übertragungseffekte.

Michael Ganske studierte Ökonomie an der Universität Augsburg. 
Intertemporale Aspekte von Staatsverschuldung und Außenhandel 


\title{
Hohenheimer Volkswirtschaftliche Schriften
}

\author{
Herausgegeben von \\ Prof. Dr. Rolf Caesar, Prof. Dr. Harald Hagemann, \\ Prof. Dr. Klaus Herdzina, Prof. Dr. Renate Ohr, Prof. Dr. Walter Piesch, \\ Prof. Dr. Ingo Schmidt, Prof. Dr. Peter Spahn, \\ Prof. Dr. Gerhard Wagenhals, \\ Prof. Dr. Helmut Walter
}

\section{Band 33}

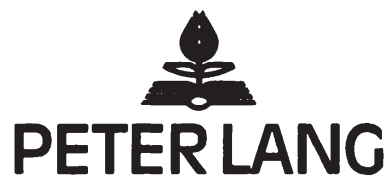

Frankfurt am Main - Berlin - Bern · New York · Paris · Wien 


\section{Michael Ganske}

\section{Intertemporale Aspekte von Staatsverschuldung und Außenhandel}

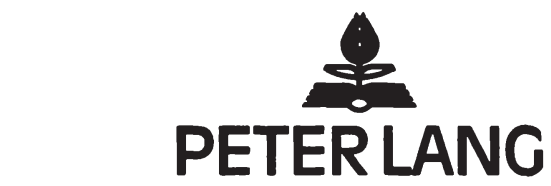

Frankfurt am Main · Berlin · Bern · New York · Parls · Wien 
Die Deutsche Bibliothek - CIP-Einheitsaufnahme

Ganske, Michael:

Intertemporale Aspekte von Staatsverschuldung und Außenhandel / Michael Ganske. - Frankfurt am Main ; Berlin ; Bern ; New York ; Paris ; Wien : Lang, 1999

(Hohenheimer Volkswirtschaftliche Schriften ; Bd. 33)

Zugl.: Hohenheim, Univ., Diss., 1998

ISBN 3-631-34627-1

Open Access: The online version of this publication is published on www.peterlang.com and www.econstor.eu under the international Creative Commons License CC-BY 4.0. Learn more on how you can use and share this work: http://creativecommons. org/licenses/by/4.0.

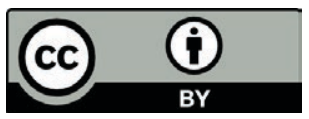

This book is available Open Access thanks to the kind support of ZBW - Leibniz-Informationszentrum Wirtschaft.

Gedruckt auf alterungsbeständigem, säurefreiem Papier.

\author{
D 100 \\ ISSN 0721-3085 \\ ISBN 3-631-34627-1 \\ ISBN 978-3-631-75472-6 (eBook) \\ (C) Peter Lang GmbH \\ Europäischer Verlag der Wissenschaften \\ Frankfurt am Main 1999 \\ Alle Rechte vorbehalten.
}

Das Werk einschließlich aller seiner Teile ist urheberrechtlich geschützt. Jede Verwertung außerhalb der engen Grenzen des

Urheberrechtsgesetzes ist ohne Zustimmung des Verlages

unzulässig und strafbar. Das gilt insbesondere für

Vervielfältigungen, Übersetzungen, Mikroverfilmungen und die

Einspeicherung und Verarbeitung in elektronischen Systemen.

Printed in Germany 123467 


\section{Vorwort}

Die Finanzierungsentscheidung des Staates und der Außenhandel wirken über verschiedene Kanäle auf die Entwicklung von Volkswirtschaften. Um eine Eruierung der Wirkungsmechanismen zu erreichen, ist eine explizite Herleitung der durch Politikentscheidungen induzierten wirtschaftlichen Dynamik notwendig. Hierfür werden mikroökonomisch fundierte dynamische makroökonomische Modelle diskutiert, anhand derer intertemporale Aspekte von Staatsverschuldung und Außenhandel aufgezeigt werden. Zur Anwendung kommen Modelle überlappender Generationen, da sie meiner Ansicht nach den geeignetsten Analyserahmen für den Untersuchungsgegenstand darstellen.

Mein Interesse an der dynamischen Wirtschaftstheorie wurde bereits während meines Studiums an der Universität Augsburg, durch den Besuch der Veranstaltungen von Herrn Professor Dr. Ulrich K. Schittko, geweckt. Die mit ihm geführten Diskussionen haben entscheidende Anregungen für diese Arbeit geliefert, wofür ich mich bedanken möchte. Gleichzeitig danke ich meinem Betreuer Herrn Professor Dr. Harald Hagemann, sowie Herrn Professor Dr. Peter Spahn für die Erstellung des Zweitgutachtens.

Hohenheim, im November 1998

Michael Ganske 
Michael Ganske - 978-3-631-75472-6

Downloaded from PubFactory at 01/11/2019 04:35:58AM

via free access 


\section{Inhaltsverzeichnis}

Darstellungsverzeichnis

1 Einfuihrung 1

$1.1 \quad$ Modelltheoretische Einordnung 3

1.2 Zum inhaltlichen Aufbau 8

2 Zwei-Länder-OLG-Modelle 11

2.1 Monetäre Zwei-Länder-OLG-Modelle 12

$\begin{array}{lll}2.1 .1 & \text { Außenhandel } & 16\end{array}$

2.1.1.1 Nicht stationäre Außenhandelsgleichgewichte 18

2.1.1.2 Die langfristigen Außenhandelsgleichgewichte 21

2.1.2 Wechselkursindeterminiertheit 22

2.2 Zwei-Länder-OLG-Modelle mit Kapitalakkumulation 24

2.2.1 Außenhandel 27

2.2.1.1 Das langfristige Außenhandelsgleichgewicht 30

2.2.1.2 Nicht stationäre Außenhandelsgleichgewichte $\quad 37$

$\begin{array}{ll}\text { 2.2.2 Fiskalpolitische Aspekte } & 39\end{array}$

2.2.2.1 Die Auswirkungen von Staatsverschuldung 39

2.2.2.1 Der Einfluß des öffentlichen Budgets auf die Auslandsposition $\quad 48$

2.2.2.3 Kapitalertragsteuern und das internationale Steuersystem 51

2.3 Zwei-Länder-OLG-Modelle mit fixem Produktionsfaktor $\quad 57$

3 Ein Modell einer OLG-Ökonomie mit Produktion 63

$\begin{array}{lll}3.1 & \text { Die Gleichgewichtsdynamik } & 65\end{array}$

3.1.1 Existenz eines langfristigen Gleichgewichts 72

$\begin{array}{ll}\text { 3.1.2 Optimalität des langfristigen Gleichgewichts } & 73\end{array}$

3.1.3 Stabilität des langfristigen Gleichgewichts 76

$\begin{array}{lll}3.2 & \text { Indeterminiertheit } & 77\end{array}$

$\begin{array}{lll}\text { 3.2.1 Die Zinsdynamik } & 82\end{array}$ 
4 Die Auswirkungen von Staatsverschuldung

4.1 Die langfristigen Auswirkungen der Staatsverschuldung 89

4.2 Die Auswirkungen eines staatlichen Budgetdefizits 94

4.2.1 Die Entwicklung der Zinssätze 96

$\begin{array}{ll}\text { 4.2.2 Die Auswirkung auf die Wohlfahrt } & 102\end{array}$

$5 \quad$ Ein Zwei-Länder-OLG-Modell der Weltwirtschaft 105

$\begin{array}{lll}5.1 & \text { Die integrierte Weltwirtschaft } & 107\end{array}$

5.2 Die langfristigen Auswirkungen von Außenhandel 114

$\begin{array}{lll}\text { 5.2.1 Die langfristige Handelsstruktur } & 116\end{array}$

5.2.1.1 Entstehung von Innengeld 117

5.2.1.2 Die Handelsbilanz 119

5.2.2 Die langfristigen nationalen Konsumpläne 121

5.2.3 Die langfristige Auswirkung auf die Wohlfahrt 123

5.3 Die Auswirkungen einer Öffnung zum Weltmarkt 127

$\begin{array}{ll}\text { 5.3.1 Die Entwicklung der Zinssätze } & 129\end{array}$

5.3.1.1 Die Entwicklung der Zinssätze aus inländischer Sicht 129

5.3.1.2 Die Entwicklung der Zinssätze aus ausländischer Sicht 131

$\begin{array}{ll}\text { 5.3.2 Die Auswirkung auf die Wohlfahrt } & 133\end{array}$

6 Staatsverschuldung im Zwei-Länder-Modell 137

6.1 Die langfristigen Auswirkungen von

Staatsverschuldung $\quad 139$

6.1.1 Der Zinssatz 142

6.1.2 Die nationalen Konsumpläne 145

$\begin{array}{ll}\text { 6.1.3 Die Handelsstruktur } & 148\end{array}$

$\begin{array}{ll}\text { 6.1.4 Die Wohlfahrt } & 149\end{array}$

6.2 Die Auswirkungen eines staatlichen Budgetdefizits 153

6.2.1 Die Entwicklung der Zinssätze 154

6.2.2 Die Auswirkung auf die Wohlfahrt 162

6.3 Die Entwicklung der Zahlungsbilanz 165 
6.3.1 Die Entwicklung der Zahlungsbilanz bei inländischer Staatsverschuldung

6.3.2 Die Entwicklung der Zahlungsbilanz bei ausländischer Staatsverschuldung

$7 \quad$ Zusammenfassung und Fazit

7.1 Zusammenfassung

7.1.1 Staatsverschuldung in der geschlossenen Ökonomie

7.1.3 Staatsverschuldung in der integrierten Weltwirtschaft

Anhang A: Das Blanchard-Modell eines kleinen Landes
A.1
Eigenschaften
A. 2
Gleichgewichtsdynamik
A. 3
Das Ricardo-Äquivalenztheorem

Anhang B: Herleitung einer Tauschkurve

Literaturverzeichnis

Stichwortverzeichnis 
Michael Ganske - 978-3-631-75472-6

Downloaded from PubFactory at 01/11/2019 04:35:58AM

via free access 


\section{Darstellungsverzeichnis}

$\begin{array}{lll}\text { Darstellung 3.1: } & \text { Dynamik }\end{array}$

Darstellung 3.2: Indeterminiertheit $\quad 79$

Darstellung 3.3: Zinsdynamik $\quad 82$

Darstellung 3.4: Zyklisches Gleichgewicht $\quad 84$

Darstellung 3.5: Zinsdynamik im zweiperiodischen Zyklus 85

Darstellung 4.1: Auswirkungen von Staatsverschuldung 91

Darstellung 4.2: $\quad$ Kurzfristiger Zinseffekt $\quad 97$

Darstellung 4.3: Mögliche Zinsdynamik 102

Darstellung 5.1: Die integrierte Weltwirtschaft 110

Darstellung 5.2: Dynamik der integrierten Weltwirtschaft 112

$\begin{array}{ll}\text { Darstellung 5.3: Welthandelsgleichgewicht } & 116\end{array}$

$\begin{array}{ll}\text { Darstellung 5.4: Handelsströme } & 118\end{array}$

Darstellung 5.5: Langfristiger Wohlfahrtseffekt von Außenhandel $\quad 124$

Darstellung 5.6: Handelsströme in Periode $1 \quad 128$

Darstellung 5.7: Zinsdynamik aus inländischer Sicht 131

Darstellung 5.8: Zinsdynamik aus ausländischer Sicht 133

Darstellung 6.1: Auswirkungen der Staatsverschuldung 141

Darstellung 6.2: Auswirkung auf die nationalen Konsumvektoren 146

Darstellung 6.3: Kurzfristiger Zinseffekt 156

Darstellung 6.4: Mögliche Zinsdynamik 161

Darstellung A.1: Gleichgewichtsdynamik 209

$\begin{array}{ll}\text { Darstellung B.1: Konsumdiagramm } & 219\end{array}$

Darstellung B.2: Auswirkung einer Diskontfaktorerhöhung 222

Darstellung B.3: Überschußnachfragediagramm 223

$\begin{array}{lll}\text { Darstellung C.1: } & \text { Offenbarte Präferenz } & 228\end{array}$ 
Michael Ganske - 978-3-631-75472-6

Downloaded from PubFactory at 01/11/2019 04:35:58AM

via free access 


\section{Einführung}

Das Anliegen dieser Abhandlung ist, intertemporale Aspekte von Staatsverschuldung und Außenhandel zu beleuchten. Als Analyserahmen hierfür dient das Modell überlappender Generationen (OLG-Modell). ${ }^{1}$ Das OLG-Modell ist ein mikroökonomisch fundiertes dynamisches makroökonomisches Modell. Die Klasse der OLG-Modelle zeichnet sich allgemein dadurch aus, daß die Akteure, die in den modellierten Ökonomien leben, eine endliche Lebenszeit besitzen und jede Periode oder zu jedem Zeitpunkt, je nachdem ob die Formulierung in diskreter Zeit oder in stetiger Zeit erfolgt, eine neue Generation geboren wird. Damit liegt eine demographische Struktur vor, die den Zeithorizont der Modelle unendlich werden läßt. Die modellierten Ökonomien besitzen somit einen unendlichen Zeithorizont, nicht aber die Akteure, die in ihnen leben. Dies unterscheidet das OLG-Modell von der zweiten Klasse mikroökonomisch fundierter dynamischer makroökonomischer Modelle, in denen sowohl die modellierten Ökonomien als auch die in ihnen lebenden Akteure einen unendlichen Zeithorizont besitzen. In dieser zweiten Modellklasse wird ein einzelner die Ökonomie bevölkernder Akteur angenommen (RA-Modell). ${ }^{2}$

Es wird nicht der Anspruch erhoben möglichst realitätsnahe Modelle der Weltwirtschaft zu generieren, sondern im Mittelpunkt steht eine auf ihre intertemporalen Aspekte reduzierte Analyse der Auswirkungen von Staatsverschuldung und Außenhandel. Neben der Darstellung der Ergebnisse aus der Literatur werden Modelle entwickelt, deren Struktur auf die für das Vorhaben notwendige Komplexität beschränkt bleibt. Die entwickelten Modelle sind in diskreter Zeit formuliert. Die Generationen leben zwei Perioden, und jede Periode wird eine neue Generation geboren. Die in das ökonomische System eintretenden Generationen sind Replikationen der vorherigen und werden jeweils durch einen ein-

\footnotetext{
${ }^{1}$ Das OLG-Modell wird mehrheitlich auf Samuelson (1958) zurückgeführt; vgl. z.B. Balasko und Shell (1980), S. 281. Es existiert allerdings auch die Auffassung, daß Maurice Allais der tatsächliche Initiator des OLG-Modells ist; vgl. Malinvaud (1995).

${ }^{2}$ Eine schöne Darstellung des RA-Modells findet sich in Turnovsky (1995), Kap. 9-13. Zum Konstrukt des repräsentativen Akteurs und seine Bedeutung in der Makroökonomik siehe Hartley (1997). Die Annahme repräsentativer Akteure wird auch kritisch gesehen; siehe z.B. Kirman (1992).
} 
zelnen Akteur repräsentiert. Von der Produktionsseite her wird eine nicht weiter spezifizierte Produktionstechnik angenommen, mit Hilfe derer es möglich ist, die Arbeitskraft unmittelbar in ein nutzenstiftendes Konsumgut zu transformieren. ${ }^{1}$ Das Produktionsergebnis fällt dabei anteilig dem Faktor Arbeit und der Produktionstechnik zu.

Die Annahme einer wie oben beschriebenen Technologie erzeugt eine entscheidende Eigenschaft der Modelle. Jede Periode gibt es zwei Zinssätze. Das ist zum einen der kurzfristige Zinssatz. Er determiniert die Verzinsung der Ersparnis der Akteure. Die Akteure sparen durch den Erwerb eines Anteils an der Produktionstechnik. Interpretiert man den Anteil des Produktionsergebnisses, welcher der Produktionstechnik zufällt, als Gewinn, so stellt der Anteilschein an der Produktionstechnik einen Vermögenstitel dar, mit dessen Eigentum ein unendlicher Dividendenstrom verbunden ist. Der unendliche Dividendenstrom ist dabei der in jeder Periode anfallende Gewinn. Der Preis des Anteilscheins ist hierbei gleich dem mit dem langfristigen Zinssatz kapitalisierten Wert des unendlichen Dividendenstroms. Da es in jeder Periode einen kurzfristigen und einen langfristigen Zinssatz gibt, ist es möglich die Auswirkungen der Staatsverschuldung und des Außenhandels auf die Zinsstruktur zu untersuchen.

Der öffentliche Sektor wird in Form eines Staates modelliert, der eine intergenerationale Umverteilungspolitik betreibt. Es findet kein öffentlicher Verbrauch von Gütern statt. Der einzige aktive Eingriff des Staates in den Wirtschaftskreislauf besteht aus Pauschaltransfers an die jungen Akteure bzw. der Erhebung einer Pauschalsteuer auf ihr Arbeitseinkommen. Der Staat finanziert ein gegebenenfalls auftretendes Budgetdefizit durch die Emission von ewigen Anleihen. Die Staatsanleihen und der Anteilschein an der Produktionstechnik sind hierbei vollkommene Substitute im Portfolio der Anleger. Somit beeinflußt die Staatsverschuldung den Preis der Vermögenstitel und die Zinssätze. Die so modellierte intergenerationale Umverteilungspolitik wird auf ihre kurzfristige und langfristige Allokationswirkung, ihren Einfluß auf die Zinsstruktur und auf die Beeinflussung der Wohlfahrt der von der Besteuerung bzw. den Transfers betroffenen Generationen untersucht.

${ }^{1}$ Die Idee zu dieser Technologie findet sich in Mas-Collel et al. (1995), S. 770. 
Die Auswirkungen von Staatsverschuldung und Außenhandel in einer integrierten Weltwirtschaft werden dann schließlich mit Hilfe eines Zwei-LänderModells untersucht. Hierfür wird einfach eine zweite Ökonomie angenommen, welche die gleiche Struktur wie die bis zu diesem Zeitpunkt analysierte aufweist. Der einzige Unterschied der beiden Länder liegt in der Zeitpräferenz der sie bevölkernden Akteure. Die Reduktion der länderspezifischen strukturellen Unterschiede auf die Zeitpräferenz ist ein natürliches Vorgehen, wenn man bedenkt, daß rein intertemporale Aspekte analysiert werden sollen. Der sich in einer unterschiedlichen Zeitpräferenz manifestierende länderspezifische Unterschied erzeugt die Notwendigkeit der Verwendung des OLG-Modells und macht etwa die Verwendung des RA-Modells wenig sinnvoll. Im letzteren Modell maximiert der repräsentative Akteur seinen mit der Zeitpräferenzrate abdiskontierten Lebensnutzen. Im langfristigen Gleichgewicht einer so modellierten Ökonomie stimmen der Zinssatz und die Zeitpräferenzrate überein. Modelliert man eine integrierte Weltwirtschaft nun durch ein Zwei-Länder-RA-Modell, so besitzt diese unter der Annahme, daß sich die Zeitpräferenzraten der Akteure in den beiden Ländern unterscheiden, kein langfristiges Gleichgewicht. Nur wenn sich der Zinssatz und die Zeitpräferenzrate entsprechen, bleibt das Konsumverhalten über die Zeit konstant (langfristiges Gleichgewicht). ${ }^{1}$ Bei sich unterscheidenden Zeitpräferenzraten, kann diese Bedingung in beiden Ländern nicht gleichzeitig erfüllt sein. ${ }^{2}$

\subsection{Modelltheoretische Einordnung}

Im Folgenden soll eine modellbezogene Einordnung der vorliegenden Abhandlung in die Literatur der mikroökonomisch fundierten dynamischen makroökonomischen Modelle erfolgen. Zusätzlich gibt der Abschnitt einen Literaturüber-

\footnotetext{
${ }^{1}$ Vgl. dazu Turnovsky (1995), S. 239f.

${ }^{2}$ Diese Feststellung wird schon in Buiter (1981) gemacht; vgl. Buiter (1981), S. 781. Im Modell von Helpman und Razin (1982) z.B. wächst das Vermögen des Landes mit dem geduldigeren Akteur ständig, und das Vermögen des Landes mit dem ungeduldigeren Akteur nimmt ständig ab. Man kann dieses Problem, das mit einer sich international unterscheidenden Zeitpräferenz einhergeht, allerdings umgehen, indem man diese endogenisiert. Siehe z.B. Obstfeld (1981) oder auch Daniel (1997) für ein solches Vorgehen.
} 
blick über Arbeiten, die sich mit Fragestellungen befassen, welche in direktem Zusammenhang mit dem Untersuchungsgegenstand der vorliegenden Abhandlung stehen.

Die Optimierungsmodelle lassen sich in zwei Klassen aufteilen. Dies ist zum einen die Klasse der OLG-Modelle und zum anderen die Klasse der RAModelle. ${ }^{1}$ Die in den Kapiteln 3 bis 6 entwickelten Modelle sind von der Produktionsseite her formal äquivalent zu OLG-Modellen, bei denen einer der Produktionsfaktoren nicht reproduzierbar ist. Ein solcher Faktor kann Boden sein. So sind zum Beispiel in den Arbeiten von Kareken und Wallace (1977) und Fried (1980) die einzigen Vermögenstitel Bodentitel. Kareken und Wallace untersuchen hierbei die Wohlfahrtseffekte einer Integration der nationalen Kapitalmärkte und Fried die Wohlfahrtseffekte von technischem Fortschritt und AuBenhandel. Auch Fried und Howitt (1988) untersuchen im Rahmen eines solchen Modells die Auswirkungen von staatlichen Budgetdefiziten auf die Wohlfahrt, die Zinssätze und die Zahlungsbilanz. Im Modell von Dornbusch (1985) wird die Produktion zwar als exogen gegeben angenommen, es befinden sich allerdings ewige Staatsanleihen im Umlauf, die als Sparmedium fungieren. Die erzeugte Modelldynamik entspricht der eines OLG-Modells mit einem nicht reproduzierbaren Produktionsfaktor. Dornbusch untersucht die Bewertung der Vermögenstitel in einem solchen Modell und die Wohlfahrtseffekte, die aus der Art der Vermögenstitel und aus dem Außenhandel resultieren.

Eine weitere Möglichkeit die Produktionsseite einer Ökonomie zu modellieren ist die, einen reproduzierbaren Produktionsfaktor anzunehmen. Dieses Vorgehen wählt Diamond (1965). Das Diamond-Modell ist ein OLG-Wachstumsmodell mit einer Ein-Sektoren-Technologie, die vermöge einer neoklassischen Produktionsfunktion repräsentiert wird. Hier entspricht die Ersparnis der jungen

\footnotetext{
${ }^{1}$ Natürlich ist das Ramsey (Cass-Koopmans)-Wachstumsmodell auch ein Optimierungsmodell. Im Rahmen des Ramsey-Modells wird der optimale Wachstumspfad einer Ökonomie untersucht. Kennzeichnend für das Ramsey-Modell ist eine positive Wachstumsrate der Bevölkerung, welche auch in der Zielfunktion der Optimierung erscheint. Formuliert man das Optimierungsproblem des Ramsey-Modells in Pro-Kopf-Größen, so gleicht die Zielfunktion der des RA-Modells. Jedoch ist die Wachstumsrate der Bevölkerung weiterhin in der Budgetrestriktion erkennbar, was das Ramsey-Modell vom RA-Modell unterscheidbar macht. Zum Ramsey-Modell siehe Ramsey (1928), Cass (1965) und Koopmans (1965).
} 
Akteure dem Kapitalstock zu Beginn der folgenden Periode. Im DiamondModell läßt sich zwar die Auswirkung der Staatsverschuldung auf den Kapitalstock einer Ökonomie analysieren, Kapitalverluste bzw. Kapitalgewinne, welche die Halter von Vermögenstiteln aufgrund einer Veränderung des langfristigen Zinssatzes hinnehmen müssen bzw. von dem sie profitieren, sind im Rahmen des Diamond-Modells allerdings nicht darstellbar. ${ }^{1}$

Von der demographischen Struktur her sind die entwickelten OLG-Modelle einfach gehalten. Die Akteure leben zwei Perioden, und jede Periode wird eine neue Generation geboren, die eine Replikation der vorherigen ist. Der Vorteil dieser Annahme ist, daß sie eine leicht zu handhabende Modelldynamik generiert und diese graphisch veranschaulicht werden kann. Im Modell von Blanchard (1985) dagegen besitzen die Akteure eine über die Lebenszeit konstante Sterbewahrscheinlichkeit, und zu jedem Zeitpunkt wird eine neue Generation von Akteuren geboren. Die Annahme bezüglich des Lebenszyklus erschwert jedoch die analytische Handhabbarkeit des Modells. Auch das BlanchardModell findet in der Literatur zur Analyse der Auswirkungen von Staatsverschuldung und Außenhandel Anwendung. ${ }^{2}$ Die Formulierung erfolgt dabei teilweise in stetiger Zeit aber auch in diskreter Zeit. ${ }^{1}$

${ }^{1}$ In der Literatur findet das Diamond-Modell zur Analyse der Auswirkungen von Fiskalpolitik und Außenhandel rege Verwendung. Persson (1985), Lin (1994a) und Tan (1997) untersuchen die Auswirkungen staatlicher Budgetdefizite. Schmid und Großmann (1986) und Schmid (1988) analysieren die Auslandsverschuldung und Buiter (1981) und Ruffin und Yoon (1993) internationale Kapitalbewegungen. Sibert (1990), Ihori (1991), Lin (1994b) und Piekkola (1995) untersuchen die Auswirkungen von Kapitalertragsteuern, Lin (1998) die Auswirkung einer Besteuerung des Arbeitseinkommens auf die Akkumulation von Humankapital und Batina und Ihori (1991) die internationalen Übertragungswirkungen von Konsumsteuern. Buiter und Kletzer (1991) schließlich untersuchen die Wohlfahrtseffekte einer internationalen Koordination der nationalen Fiskalpolitik.

${ }^{2}$ Itaya (1995) untersucht die langfristige Steuerinzidenz. Nielsen (1992) und Christensen und Nielsen (1995) analysieren die internationalen Übertragungswirkungen von Kapitalertragsteuern und Summers (1981) und Burbidge und Scarth (1995) ihre Wohlfahrtseffekte. Iwamoto und Shibata (1991) und Bovenberg $(1992,1994)$ untersuchen die Auswirkung der Kapitalertragsteuer auf die Leistungsbilanz unter dem Aspekt des Steuersystems (Besteuerung nach dem Wohnsitzstaatprinzip vs. Besteuerung nach dem Quellenstaatprinzip). Frenkel und Razin $(1986 \mathrm{a}, 1986 \mathrm{~b})$ untersuchen die internationalen Übertragungswirkungen von Staatsausgaben und Buiter (1987) und Frenkel und Razin (1987) die der Staatsverschuldung. Bovenberg und 
Das Modell von Weil (1989) stellt neben dem Blanchard-Modell eine weitere wichtige Variante des OLG-Modells dar. Im Weil-Modell werden zu jedem Zeitpunkt neue Generationen geboren, deren Lebenszeit unendlich ist. ${ }^{2}$ Auch das Weil-Modell besitzt für das OLG-Modell typische Eigenschaften (Möglichkeit der dynamischen Ineffizienz von Walras-Gleichgewichten, Existenz von spekulativen Preisblasen und Nichtgültigkeit des Ricardo-Äquivalenztheorems) und findet in der Literatur als Analyserahmen Verwendung. ${ }^{3}$

Das OLG-Modell läßt sich auch um ein Vermächtnismotiv der Generationen erweitern. Der Nutzen einer Generation wird dann positiv vom Nutzen der ihr folgenden Generation beeinflußt. ${ }^{4}$ Dieser auf Barro (1974) zurückgehende Ansatz führt allerdings dazu, daß sich eine so modellierte OLG-Ökonomie unter Umständen wie eine RA-Ökonomie verhält. ${ }^{5}$ Im RA-Modell wird ein repräsen-

Ewijk (1997) schließlich untersuchen die Auswirkungen eines progressiven Steuersystems auf die Akkumulation von Humankapital.

'Eine exzellente Diskussion intertemporaler Aspekte der Fiskalpolitik, im Rahmen eines in diskreter Zeit formulierten Blanchard-Modells, findet sich in Frenkel und Razin (1996), Kap. 9-10.

${ }^{2}$ Das Weil-Modell entspricht nicht der zu Beginn genannten allgemeinen Charakteristik einer begrenzten Lebenszeit der Akteure. Da das Weil-Modell die einzige Ausnahme dieser Regel darstellt, scheint die zu Beginn getroffene allgemeine Charakterisierung des OLG-Modells dennoch gerechtfertigt.

${ }^{3}$ Nielsen und Sorensen $(1991,1994)$ z.B. untersuchen, unter Verwendung des Weil-Modells, die internationalen Übertragungswirkungen von Kapitalertragsteuern und Alogoskoufis und van der Ploeg (1991) jene von Staatsverschuldung.

${ }^{4}$ Steigum (1993) untersucht, im Rahmen eines solchen OLG-Modells, die langfristigen Auswirkungen von Fiskalpolitik in einer kleinen offenen Volkswirtschaft und Renström (1996) versucht eine Endogenisierung der Fiskalpolitik. Zilcha (1998) analysiert die Auswirkungen verschiedener Manifestationen des Vermächtnismotivs (Investition in die Ausbildung der Nachkommen vs. Vermögenstransfers) auf das gesamtwirtschaftliche Wachstum. In diesem Zusammenhang sei hier auch auf Caballe (1998) hingewiesen. Caballe untersucht die Wachstumseffekte von Kapitalertragsteuer und Lohnsteuer. Die optimale Besteuerung hängt davon $a b$, ob das Vermächtnismotiv operativ wird oder nicht. $\mathrm{Ob}$ es operativ wird hängt wiederum von den Steuersätzen ab.

${ }^{5}$ So untersuchen Barro (1974) und Weil (1987), im Rahmen eines solchen Modells, die Gültigkeit des Ricardo-Äquivalenztheorems. Barro zeigt, daß das Äquivalenztheorem Gültigkeit besitzt, was eine typische Eigenschaft des RA-Modells ist. Weil dagegen zeigt, daß das Vermächtnismotiv in der Regel zu schwach ist, tatsächlich also nicht operativ wird, und so das Äquivalenztheorem keine Gültigkeit besitzt. Jaeger (1998) untersucht den Einfluß endogen bestimmter intragenerational heterogener Altruismusausprägungen (diese determinieren das 
tativer Akteur mit unendlicher Lebenszeit angenommen. Die Formulierung erfolgt hierbei überwiegend in stetiger Zeit, teilweise aber auch in diskreter Zeit. Eine wichtige Eigenschaft des RA-Modells ist, daß in ihm das Ricardo-Äquivalenztheorem gilt. Das Ricardo-Äquivalenztheorem besagt, daß das bei einem gegebenen Niveau der Staatsausgaben resultierende ökonomische Gleichgewicht nicht davon beeinflußt wird, ob der Staat seine Ausgaben über Steuern oder Schulden finanziert. Anders formuliert ist die Gleichgewichtsallokation unabhängig vom Pfad der Besteuerung und der staatlichen Budgetsalden. ${ }^{1} \mathrm{Da}$ das Ricardo-Äquivalenztheorem im RA-Modell Gültigkeit besitzt, ist eine Analyse der Auswirkungen staatlicher Budgetdefizite innerhalb dieses Modells wenig sinnvoll. Andere Aspekte von Fiskalpolitik und Außenhandel lassen sich aber sehr wohl analysieren. ${ }^{2}$

Vermächtnis- bzw. Schenkungsverhalten der Akteure) auf die Gültigkeit des Äquivalenztheorems.

${ }^{1}$ Die Idee, die hinter dem Äquivalenztheorem steckt ist die, daß die Finanzierungsentscheidung des Staates (Steuer- vs. Schuldenfinanzierung) keinen Einfluß auf die Lebensbudgetrestriktion des Akteurs besitzt. Eine in der Gegenwart stattfindende Reduktion der Besteuerung geht mit einer erhöhten staatlichen Verschuldung einher (Budgetdefizit). Diese erfordert eine erhöhte Besteuerung in der Zukunft, da der Staat seine intertemporale Budgetrestriktion einhalten muß. In Gegenwartsgrößen ausgedrückt hat sich die steuerliche Belastung des Akteurs nicht verändert. Dies zeigt auch, warum das Ricardo-Äquivalenztheorem im OLG-Modell nicht gelten muß. Hier ist es möglich, daß die von einer Steuerreduktion profitierenden Akteure nicht die selben sind, die von der notwendigen zukünftigen Erhöhung der Steuer getroffen werden.

${ }^{2}$ So untersucht Lucas (1990) die optimale Ausgestaltung von Kapitalertragsteuer und Lohnsteuer und Bianconi (1995) ihre internationalen Übertragungswirkungen. Frenkel und Razin (1985a,1985b) analysieren die Auswirkung von Staatsausgaben auf den Zinssatz und ihre internationale Übertragungswirkungen. Bianconi und Turnovsky (1997) untersuchen, unter dem Aspekt alternativer Steuern, die internationalen Übertragungswirkungen von Staatsausgaben. Sachs (1982) beschäftigt sich mit intertemporalen Aspekten der Leistungsbilanzbestimmung und Devereux und Shi (1991) mit dem Zusammenhang zwischen Kapitalakkumulation und Leistungsbilanzdynamik. Mansoorian (1998) untersucht den Einfluß der intratemporalen Terms of Trade auf die Leistungsbilanz und den J-Kurven-Effekt. Zwei empirische Analysen bieten Asea und Turnovsky (1998), die die Wachstumseffekte von Kapitalertragsteuern und ihren Einfluß auf die privaten Portfolio-Entscheidungen untersuchen, und Dahan und Hercowitz (1998), die den Einfluß von Konsum- und Einkommensteuer auf das Sparverhalten analysieren. Es sei hier auch auf Turnovsky (1997) verwiesen, in dem eine exzellente Diskussion von RA-Modellen, unter dem Aspekt außenwirtschaftstheoretischer Fragestellungen, stattfindet. 


\subsection{Zum inhaltlichen Aufbau}

Im Folgenden soll der Aufbau der Abhandlung skizziert werden. Kapitel 2 gibt einen Überblick über die Literatur, die sich im Rahmen von Zwei-Länder-OLGModellen mit außenwirtschaftstheoretischen und fiskalpolitischen Fragestellungen befaßt. Die Darstellung bleibt dabei allerdings auf Modelle zwei Perioden lebender Akteure beschränkt, das Blanchard- und das Weil-Modell finden keine Berücksichtigung. ${ }^{1}$ Die Modelle werden nach dem Medium, mittels dem die Akteure sparen, gruppiert. In Abschnitt 2.1 werden monetäre Zwei-LänderOLG-Modelle vorgestellt. Hier sparen die Akteure in Form von Zentralbankgeld. Es wird die Außenhandels- und Wechselkursdynamik skizziert. In Abschnitt 2.2 werden Zwei-Länder-OLG-Modelle mit Kapitalakkumulation vorgestellt. In solchen Modellen sparen die Akteure in Form von Produktivkapital. Hier werden die Außenhandelsdynamik und fiskalpolitische Aspekte, wie Staatsverschuldung, Steuern und Staatskonsum, diskutiert. In Abschnitt 2.3 schließlich werden die Zwei-Länder-OLG-Modelle angesprochen, bei denen ein Produktionsfaktor nicht reproduzierbar ist. Das Sparmedium sind hier Anteilscheine an dem fixen Produktionsfaktor. Da die Modelle der folgenden Kapitel formal äquivalent zu diesen Modellen sind und dort eine ausführliche Diskussion stattfindet, ist dieser Abschnitt kurz gehalten.

Kapitel 3 stellt den Modellrahmen vor, innerhalb dessen die weitere Analyse stattfindet. Der staatliche Sektor findet dabei noch keine Berücksichtigung. Das Ziel dieses Kapitels ist es, eine komplette Beschreibung der aus den Modellannahmen resultierenden Gleichgewichtsdynamik zu liefern (Abschnitt 3.1). Hierfür wird die Existenz, die Optimalität, sowie die Stabilität des langfristigen Gleichgewichts der modellierten Ökonomie diskutiert. Anschließend wird die Eigenschaft der Indeterminiertheit untersucht (Abschnitt 3.2). Diese ist deshalb von Bedeutung, da sie mit der Möglichkeit der Existenz von zyklischen Gleichgewichten einhergeht. Auch findet eine Herleitung der im Modell erzeugten Zinsdynamik statt.

1 Das Blanchard-Modell unterscheidet sich durch seine demographische Struktur von den OLG-Modellen, die in den Kapiteln 2 bis 6 diskutiert werden. Da das Blanchard-Modell eine wichtige Variante des OLG-Modells ist, findet im Anhang A eine in stetiger Zeit formulierte Darstellung des Blanchard-Modells eines kleinen Landes statt. 
In Kapitel 4 wird die Struktur des Modells um einen staatlichen Sektor erweitert, der Umverteilungspolitik betreibt. Diese Politik erzeugt ein Budgetdefizit, welches die Emission von Staatsanleihen notwendig macht. Es wird nun untersucht, wie sich die Staatsverschuldung kurz- und langfristig auf die Ökonomie auswirkt. Dabei interessieren drei Aspekte. Diese sind die Allokation, die Zinssätze und die Wohlfahrt der durch die Schuldenpolitik betroffenen Generationen. In Abschnitt 4.1 werden die langfristigen Auswirkungen der Staatsverschuldung eruiert. Hierfür wird das langfristige Gleichgewicht einer Ökonomie mit einem verschuldeten Staat dem der Ökonomie ohne Staatsverschuldung gegenübergestellt. In Abschnitt 4.2 wird die durch ein staatliches Budgetdefizit induzierte Gleichgewichtsdynamik analysiert. Es wird also der Entwicklungspfad modelliert, welcher die Ökonomie vom langfristigen Gleichgewicht ohne Staatsverschuldung hin zum langfristigen Gleichgewicht mit positiver Staatsverschuldung führt.

Kapitel 5 erweitert das Modell auf zwei Länder. Es wird also ein Zwei-LänderOLG-Modell der Weltwirtschaft generiert. Zu diesem Zweck wird die Existenz einer weiteren OLG-Ökonomie angenommen, die sich von der in den vorherigen Kapiteln analysierten nur bezüglich der intertemporalen Nutzenfunktion der sie bevölkernden Akteure unterscheidet. Der Unterschied liegt hierbei in der Zeitpräferenz der die beiden Ökonomien bevölkernden Akteure. In Abschnitt 5.1 wird das langfristige Gleichgewicht der integrierten Weltwirtschaft beschrieben, also das Gleichgewicht, das resultiert wenn Freihandel herrscht. In Abschnitt 5.2 werden die langfristigen Auswirkungen des Außenhandels auf die nationalen Konsumpläne, die Wohlfahrt der Akteure in beiden Ländern und den Zinssatz analysiert. Hierbei erfolgt eine explizite Analyse der langfristigen Welthandelsstruktur. In Abschnitt 5.3 findet eine ausführliche Darstellung der Auswirkungen einer Öffnung der Ökonomien zum Weltmarkt statt. Es wird also die Entwicklung der Weltwirtschaft modelliert, die sie vom Autarkiegleichgewicht (hier bestehen keine wirtschaftlichen Beziehungen zwischen den Ländern) zum langfristigen Freihandelsgleichgewicht bringt. Hierbei erfolgt eine Analyse der durch die Öffnung zum Weltmarkt induzierten Zinsdynamik, sowie eine Bewertung der Wohlfahrt der vom Integrationsprozeß betroffenen Generationen. 
Kapitel 6 schließlich führt die in den Kapiteln 4 und 5 gewonnenen Erkenntnisse zusammen. Es wird untersucht, wie sich die Staatsverschuldung in der integrierten Weltwirtschaft auswirkt. In Abschnitt 6.1 erfolgt die Analyse der langfristigen Auswirkungen der Staatsverschuldung. Hierbei wird das langfristige Gleichgewicht der integrierten Weltwirtschaft ohne Staatsverschuldung einem langfristigen Gleichgewicht der integrierten Weltwirtschaft mit positiver Staatsverschuldung gegenübergestellt. Dabei wird angenommen, daß sich nur der Staat eines Landes verschuldet, während der des anderen Landes weiterhin unverschuldet ist. Der Sinn dieses Vorgehens ist, die internationalen Übertragungswirkungen der Staatsverschuldung klarer herauszuarbeiten. Bei der Analyse interessieren die Auswirkungen auf den Zinssatz, die nationalen Konsumpläne, die Handelsstruktur und die Wohlfahrt. In Abschnitt 6.2 wird untersucht wie sich ein in einem Land auftretendes staatliches Budgetdefizit in der integrierten Weltwirtschaft auswirkt. Es wird also der Pfad analysiert, der die integrierte Weltwirtschaft vom langfristigen Gleichgewicht ohne Staatsverschuldung hin zum langfristigen Gleichgewicht mit positiver Staatsverschuldung bringt. Es interessieren hier wieder die durch das Budgetdefizit induzierte Zinsdynamik und die Wohlfahrt der von ihm betroffenen Generationen. In Abschnitt 6.3 wird die durch das Budgetdefizit erzeugte Zahlungsbilanzdynamik dargestellt. Hierbei interessiert die Entwicklung der Leistungsbilanz und die der Handelsbilanz.

Da die in den Modellen erzeugten Auswirkungen der Staatsverschuldung und des Außenhandels entscheidend vom Kapitalverlust bzw. Kapitalgewinn, den die Halter von Vermögenstiteln hinnehmen müssen bzw. von dem sie profitieren, determiniert werden, findet jeweils ein Vergleich der in den Kapiteln 4 bis 6 erhaltenen Ergebnisse mit denen, die im Diamond-Modell resultieren, statt. Im Diamond-Modell gibt es keinen Kapitalgewinn oder Kapitalverlust, dafür wird aber die Entwicklung des Kapitalstocks modelliert. Der unmittelbare Vergleich der in beiden Modellen erzeugten Ergebnisse birgt den Vorteil, daß zum einen die Bedeutung von Kapitalgewinn und Kapitalverlust klar herausgearbeitet werden kann und zum anderen die intertemporalen Aspekte der Kapitalakkumulation dargestellt werden. In Kapitel 7 schließlich erfolgt eine Zusammenfassung der in den Kapiteln 3 bis 6 hergeleiteten Ergebnisse. Außerdem wird ein Fazit aus den in der Abhandlung dargestellten Erkenntnissen gezogen. 


\section{Zwei-Länder-OLG-Modelle}

In diesem Kapitel soll ein Überblick über die Literatur gegeben werden, die sich, im Rahmen von Zwei-Länder-OLG-Modellen, mit intertemporalen Aspekten des Außenhandels und der Finanzierungsentscheidung des Staates beschäftigt. Es lassen sich hierbei drei Modellgruppen unterscheiden. Die erste Gruppe ist die der monetären OLG-Modelle. In monetären OLG-Ökonomien wird in Form von Zentralbankgeld gespart. Es handelt sich hierbei um reine Tauschmodelle. Die zweite Gruppe ist die der OLG-Modelle mit Kapitalakkumulation. Hier sparen die Akteure in Form von Produktivkapital. Die dritte Gruppe umfaßt ebenfalls Produktionsmodelle, allerdings mit einem nicht reproduzierbaren Produktionsfaktor. Das Sparmedium sind hier Anteilscheine an dem fixen Produktionsfaktor.

Die Aufteilung der Klasse der Zwei-Länder-OLG-Modelle nach dem Medium, mittels dessen die Akteure sparen, ist deswegen sinnvoll, da im Rahmen der vorliegenden Arbeit intertemporale Aspekte betrachtet werden sollen. Ein genuin intertemporales Phänomen ist Sparen. ${ }^{1}$ Es zeigt sich, daß unterschiedliche Sparprozesse zu sich unterscheidenden Ergebnissen führen.

Die im weiteren Verlauf betrachteten Weltwirtschaften bestehen aus zwei Ökonomien, von denen die eine Inland genannt wird und die andere Ausland. Charakteristika der betrachteten Ökonomien werden in Kleinbuchstaben geschrieben. Unterscheidet sich das Ausland vom Inland in einer Eigenschaft, so wird diese spezifische Charakteristik des Auslands in Großbuchstaben geschrieben.

\footnotetext{
${ }^{1}$ Dies ist mit ein Grund, warum die Verwendung des OLG-Modells sinnvoller ist, als die des RA-Modells. In einer OLG-Ökonomie gibt es in jeder Periode Akteure die sparen und Akteure die entsparen. Dies ist eine Eigenschaft, die auch reale Ökonomien aufweisen. In einer RAÖkonomie wird entweder gespart oder entspart. Beides ist gleichzeitig nicht möglich, was eine wenig realistische Eigenschaft ist.
} 


\subsection{Monetäre Zwei-Länder-OLG-Modelle}

Monetäre OLG-Ökonomien zeichnen sich dadurch aus, daß die Akteure, die in ihnen leben, in Form von Zentralbankgeld (Außengeld) sparen. Wird im folgenden von Geld gesprochen, so ist damit Zentralbankgeld gemeint. Zur Veranschaulichung sei das Modell von Schittko und Eckwert (1988) betrachtet.

Die Weltwirtschaft besteht aus zwei OLG-Ökonomien. Ihre Geschichte wird durch die diskreten Perioden $t \in Z$ indiziert. Sie beginnt in $-\infty$ und reicht bis $+\infty$. Jede Periode wird eine neue Generation von repräsentativen Akteuren (jeweils ein repräsentativer Inländer und ein repräsentativer Ausländer) geboren, die zwei Perioden leben und durch ihre erste Lebensperiode indiziert werden. Die die Weltwirtschaft bevölkernden Akteure werden durch ihre Präferenzordnung und die ihnen zukommende Erstausstattung charakterisiert. Die Generationen sind Replikationen. Somit sind die Präferenzordnung und die Erstausstattung der Inländer bzw. die der Ausländer über die Zeit konstant. Die Akteure erhalten eine Erstausstattung eines verderblichen nutzenstiftenden Konsumgutes. ${ }^{1}$ Der Ausstattungsvektor des repräsentativen Inländers der Generation $t$ ist $w=\left(w_{t}^{t}, w_{t+1}^{t}\right)$, der des repräsentativen Ausländers $W=\left(W_{t}^{t}, W_{t+1}^{t}\right)$. Die erste Komponente eines Ausstattungsvektors gibt die Jugendausstattung an, die zweite Komponente die Altersausstattung. Das Konsumgut ist nur in der Periode der Zuweisung konsumierbar. Die Präferenzordnung der Akteure kann vermöge einer intertemporalen Nutzenfunktion repräsentiert werden. Die intertemporale Nutzenfunktion des repräsentativen Inländers der Generation $t$ ist $u\left(c_{t}^{t}, c_{t+1}^{t}\right)$ und die des repräsentativen Ausländers $U\left(C_{t}^{t}, C_{t+1}^{t}\right)$. Das erste Argu-

${ }^{1}$ Die Verderblichkeit der Güter ist die in monetären OLG-Modellen übliche Annahme um die Verwendung von Geld zu motivieren. Die einzige Funktion, die Geld in solchen OLG-Ökonomien erfüllen kann, ist die Wertaufbewahrung. Die Eigenschaften des Geldes als Transaktionsmedium und Recheneinheit werden nicht erfaßt. Tobin (1980) kritisiert aus diesem Grund die Verwendung des OLG-Modells, zur Erklärung der Existenz von Geld; vgl. Tobin (1980), S. 85. Folgende Überlegung von Burke (1987) entkräftet die Kritik von Tobin: Die Wertaufbewahrungsfunktion bezieht sich auf den Transfer von Vermögen über einen Zeitraum, die Tauschmittelfunktion auf den Transfer von Vermögen zu einem Zeitpunkt. Da keine Transaktion wirklich gleichzeitig stattfindet, ist die Unterscheidung zwischen Tauschmittel- und Wertaufbewahrungsfunktion, bei sehr kurzen Periodenlängen, allerdings arbiträr; vgl. Burke (1987), S. 203. 
ment der Nutzenfunktionen ist jeweils der Jugendkonsum und das zweite Argument der Alterskonsum. Die Nutzenfunktionen sind streng quasikonkav, differenzierbar und streng ansteigend. Des weiteren sind die Ökonomien des Inlands und des Auslands vom Samuelson-Typ. Somit wollen die Akteure in beiden Ländern, bei einem intertemporalen Güterpreisverhältnis von eins, sparen. ${ }^{1}$

Sei $p_{t}$ der inländische Nominalpreis des Konsumgutes, in Periode $t$, und $P_{t}$ der ausländische. Seien $p_{t+1}^{e}$ bzw. $P_{t+1}^{e}$ die für Periode $t+1$ erwarteten Güterpreise. Es wird unterstellt, daß die Akteure vollkommene Voraussicht besitzen. Somit sind die erwarteten Güterpreise gleich den tatsächlichen Güterpreisen. Also gilt $p_{t+1}^{e}=p_{t+1}$ und $P_{t+1}^{e}=P_{t+1}, \forall t$. Seien die intertemporalen Güterpreisverhältnisse als $q_{t}:=p_{t} / p_{t+1}$ und $Q_{t}:=P_{t} / P_{t+1}$ definiert. Das intertemporale Nutzenmaximierungsproblem der Inländer lautet dann:

$$
u\left(c_{t}^{t}, c_{t+1}^{t}\right) \rightarrow \underset{c_{t}^{t}, c_{t+1}^{t}}{\operatorname{Max} !}
$$

unter der $N B$

$$
\begin{aligned}
c_{t}^{t}+m_{d}^{t} & =w_{t}^{t} \\
c_{t+1}^{t} & =w_{t+1}^{t}+q_{t} \cdot m_{d}^{t}
\end{aligned}
$$

(Budgetrestriktion Jugend)

(Budgetrestriktion Alter)

In der Jugend konsumieren die Akteure einen Teil ihrer Jugendausstattung und sparen gegebenfalls einen Teil. Dieser Sparwunsch äußert sich in der realen Geldnachfrage $m_{d}^{t}$. Im Alter setzt sich der Konsum zum einen aus der Ausstattungskomponente $w_{t+1}^{t}$ und zum anderen aus der Ersparniskomponente $q_{t} \cdot m_{d}^{t}$ zusammen. Man erkennt, daß sich das intertemporale Güterpreisverhältnis als Verzinsung der Ersparnis interpretieren läßt. Herrscht Inflation $\left(q_{t}<1\right)$, verliert die Ersparnis an Wert (Kaufkraftverlust). Herrscht Deflation $\left(q_{t}>1\right)$, gewinnt die Ersparnis an Wert (Kaufkraftgewinn).

Die Jugendbudgetrestriktion und die Altersbudgetrestriktion lassen sich in die intertemporale Lebensbudgetrestriktion transformieren:

$$
q_{t} \cdot c_{t}^{t}+c_{t+1}^{t}=q_{t} \cdot w_{t}^{t}+w_{t+1}^{t}
$$

${ }^{1}$ Zur Unterscheidung Samuelson-Ökonomie vs. klassische Ökonomie vgl. Gale (1973), S. $20 \mathrm{f}$. 
(2.2) ist die in Zukunftspreisen ausgedrückte intertemporale Budgetrestriktion. $q$, läßt sich somit alternativ als Zukunftspreis des Gegenwartskonsums interpretieren.

Die zu maximierende Lagrangefunktion lautet:

$$
L=u\left(c_{t}^{t}, c_{t+1}^{t}\right)+\lambda \cdot\left(q_{t} \cdot w_{t}^{t}+w_{t+1}^{t}-q_{t} \cdot c_{t}^{t}-c_{t+1}^{t}\right)
$$

Aus den Bedingungen erster Ordnung folgt:

$$
\frac{\frac{\partial u\left(c_{t}^{t}, c_{t+1}^{t}\right)}{\partial c_{t}^{t}}}{\frac{\partial u\left(c_{t}^{t}, c_{t+1}^{t}\right)}{\partial c_{t+1}^{t}}}=q_{t} \stackrel{\text { Budgetres. }}{\longrightarrow} \text { optimaler Konsumvektor }\left(c_{t}^{t}, c_{t+1}^{t}\right)
$$

Das optimale Konsumprogramm zeichnet sich dadurch aus, daß die Grenzrate der Substitution im Konsum mit dem intertemporalen Güterpreisverhältnis (= die ökonomische Substitutionsrate) übereinstimmt.

Sei $z_{t}^{t}:=c_{t}^{t}-w_{t}^{t}$ die Überschußnachfrage in der Jugend und $z_{t+1}^{t}:=c_{t+1}^{t}-w_{t+1}^{t}$ die im Alter. Die Überschußnachfrage gibt die Differenz zwischen Konsumnachfrage und Erstausstattung an. Überschußnachfragefunktionen sind stetig, erfüllen das Walras-Gesetz und sind homogen nullten Grades in den Preisen. ${ }^{1}$ Somit sind die Überschußnachfragefunktionen nur eine Funktion des intertemporalen Preisverhältnisses. Es gilt also:

$$
q_{t} \cdot z_{t}^{t}\left(q_{t}\right)+z_{t+1}^{t}\left(q_{t}\right) \equiv 0 . \quad \text { (Walras-Gesetz) }
$$

Das Walras-Gesetz besagt, daß der Wert der Überschußnachfragen identisch null ist. (2.5) ist also bei allen $q_{t}$ erfüllt. Die reale inländische Geldnachfrage in Periode $t$ ist somit

\footnotetext{
${ }^{1}$ Vgl. Farmer (1993), S. 64ff.
} 


$$
m_{d}^{t}\left(q_{t}\right)=-z_{t}^{t}\left(q_{t}\right)=\frac{1}{q_{t}} \cdot z_{t+1}^{t}\left(q_{t}\right)
$$

Die reale inländische Geldnachfrage ist in jeder Periode gleich dem Überschußangebot des jungen Inländers, welches gleich dem Gegenwartswert seiner Überschußnachfrage im Alter ist ( $q_{t}^{-1}$ ist der Gegenwartspreis des Zukunftskonsums).

Das analog zu interpretierende intertemporale Nutzenmaximierungsproblem der Ausländer lautet:

$$
U\left(C_{t}^{t}, C_{t+1}^{t}\right) \rightarrow \underset{C_{t}^{t}, C_{t+1}^{t}}{\operatorname{Max} !}
$$

unter der $N B$

$$
\begin{aligned}
C_{t}^{t}+M_{d}^{t} & =W_{t}^{t} \\
C_{t+1}^{t} & =W_{t+1}^{t}+Q_{t} \cdot M_{d}^{t}
\end{aligned}
$$

(Budgetrestriktion Jugend)

(Budgetrestriktion Alter)

Die intertemporale Budgetrestriktion der Ausländer lautet damit:

$$
Q_{t} \cdot C_{t}^{t}+C_{t+1}^{t}=Q_{t} \cdot W_{t}^{t}+W_{t+1}^{t}
$$

und es gilt:

$$
\frac{\frac{\partial U\left(C_{t}^{t}, C_{t+1}^{t}\right)}{\partial C_{t}^{t}}}{\frac{\partial U\left(C_{t}^{t}, C_{t+1}^{t}\right)}{\partial C_{t+1}^{t}}}=Q_{t} \stackrel{\text { Budgeres. }}{\longrightarrow} \text { optimaler Konsumvektor }\left(C_{t}^{t}, C_{t+1}^{t}\right)
$$

Auch die Überschußnachfragen der Ausländer erfüllen das Gesetz von Walras:

$$
Q_{t} \cdot Z_{t}^{t}\left(Q_{t}\right)+Z_{t+1}^{t}\left(Q_{t}\right) \equiv 0 . \quad \text { (Walras-Gesetz) }
$$

Die reale ausländische Geldnachfrage in Periode $t$ ist damit 


$$
M_{d}^{t}\left(Q_{t}\right)=-Z_{t}^{t}\left(Q_{t}\right)=\frac{1}{Q_{t}} \cdot Z_{t+1}^{t}\left(Q_{t}\right)
$$

\subsubsection{Außenhandel}

Das intertemporale Preisverhältnis gibt die Verzinsung der Ersparnis an. In einer Welt ohne Handelshemmnisse ist es unmöglich, daß die Ersparnis in einem Land rentabler ist als in einem anderen. Ansonsten wäre die Möglichkeit von Arbitragegeschäften gegeben. ${ }^{1}$ Ist der Güterhandel zwischen den beiden Ländern ohne Hemmnisse möglich, müssen im Walras-Gleichgewicht das intertemporale Preisverhältnis im Inland und im Ausland in jeder Periode gleich sein (Arbitragefreiheit). Es gilt also $q_{t}=Q_{t}, \forall t$. Seien die Weltüberschußnachfragefunktion der jungen Akteure als

$$
\xi_{t}^{t}\left(q_{t}\right):=z_{t}^{t}\left(q_{t}\right)+Z_{t}^{t}\left(q_{t}\right)
$$

und die Weltüberschußnachfragefunktion der alten Akteure als

$$
\xi_{t+1}^{t}\left(q_{t}\right):=z_{t+1}^{t}\left(q_{t}\right)+Z_{t+1}^{t}\left(q_{t}\right)
$$

definiert. Der Weltgütermarkt befindet sich in Periode $t$ im Gleichgewicht, wenn das Weltüberschußangebot der Jungen dieser Periode gleich der Weltüberschußnachfrage der Alten dieser Periode ist:

$$
-\xi_{t}^{t}\left(q_{t}\right)=\xi^{t-1}\left(q_{t-1}\right)
$$

${ }^{1}$ Bei einer sich international unterscheidenden Verzinsung der Ersparnis ist es optimal, sich in dem Land mit dem niedrigeren Zinssatz zu verschulden und das aufgenommene Geld in dem Land mit dem höheren Zinssatz anzulegen. Mit diesem Arbitragegeschäft lassen sich unbegrenzt Gewinne generieren. Eine solche Situation kann kein Gleichgewicht sein. Das Tätigen von Arbitragegeschäften erzeugt sich international angleichende intertemporale Preisverhältnisse. Erst wenn diese sich angeglichen haben, sind keine Arbitragemöglichkeiten mehr gegeben. 
Um die Gleichgewichtsbedingung der nationalen Geldmärkte zu formulieren, ist es notwendig die Handelsbilanz zu definieren. Die Handelsbilanz der Periode $i$ sei definiert als

$$
H_{1}\left(q_{1}, q_{i-1}\right):=z_{i}^{\prime}\left(q_{i}\right)+z_{1}^{i-1}\left(q_{1-1}\right) .
$$

Ist die Summe aus der Überschußnachfrage des jungen Inländers und der Überschußnachfrage des alten Inländers positiv, und somit $H_{i}$ größer als 0 , so besitzt das Inland ein Handelsbilanzdefizit. Ist die Summe der Überschußnachfragen negativ, liegt im Inland also ein gesamtwirtschaftliches Überschußangebot vor, so besitzt das Inland einen Handelsbilanzüberschuß. Wie aus der Gleichgewichtsbedingung (2.14) folgt, läßt sich die Handelsbilanz äquivalent schreiben als

$$
H_{i}\left(q_{i}, q_{i-1}\right)=-\left[Z_{i}^{i}\left(q_{i}\right)+Z_{i}^{i-1}\left(q_{i-1}\right)\right] .
$$

Einem inländischen Handelsbilanzdefizit (Handelsbilanzüberschuß) muß natürlich ein ausländischer Handelsbilanzüberschuß (ausländisches Handelsbilanzdefizit) in gleicher Höhe gegenüber stehen. Hat das Inland in Periode $i$ zum Beispiel ein Handelsbilanzdefizit, ist $H_{i}$ also positiv, so muß die Summe aus der Überschußnachfrage des jungen Ausländers und der Überschußnachfrage des alten Ausländers negativ sein. Im Ausland muß also ein gesamtwirtschaftliches Überschußangebot in gleicher Höhe vorliegen.

Nun ist man in der Lage die Gleichgewichte auf den nationalen Geldmärkten zu formulieren. Die Gleichgewichtsbedingungen für den inländischen und den ausländischen Geldmarkt der Periode $t$ lauten:

$$
\begin{aligned}
& m_{d}^{\prime}\left(q_{t}\right)=\frac{1}{p_{t}} \cdot\left[m-\sum_{i=-\infty}^{t} p_{i} \cdot H_{i}\left(q_{i}, q_{i-1}\right)\right], \\
& M_{d}^{t}\left(q_{t}\right)=\frac{1}{P_{t}} \cdot\left[M+\sum_{i=-\infty}^{t} P_{i} \cdot H_{i}\left(q_{i}, q_{i-1}\right)\right] .
\end{aligned}
$$


$m$ ist die von der inländischen Zentralbank in Umlauf gebrachte Geldmenge (autonomes inländisches Geldangebot) und $M$ die von der ausländischen Zentralbank in Umlauf gebrachte Geldmenge (autonomes ausländisches Geldangebot). Die Summenausdrücke geben die kumulierten Handelsbilanzen der vergangenen und der gegenwärtigen Periode an, bewertet mit dem Güterpreis der jeweiligen Perioden. Hat das Inland in Periode $i$ ein Handelsbilanzdefizit, gilt also $H_{i}>0$, so müssen die importierten Güter bezahlt werden, was ein Abfluß an Geld darstellt. Hat das Inland in Periode $i$ einen Handelsbilanzüberschuß, gilt also $H_{i}<0$, so muß das Ausland die exportierten Güter bezahlen, was ein Zufluß an Geld bewirkt. Ganz analog erfolgt die Interpretation der Gleichgewichtsbedingung (2.18), also die des ausländischen Geldmarktes. Ein inländisches Handelsbilanzdefizit entspricht einem ausländischen Handelsbilanzüberschuß, dort wird somit die Geldmenge vergrößert. Ein inländischer Handelsbilanzüberschuß entspricht einem ausländischen Handelsbilanzdefizit, somit reduziert sich in diesem Fall die Geldmenge im Ausland. Die Nachfrage nach Geld, die der junge Akteur des jeweiligen Landes aufgrund seiner Sparentscheidung entfaltet, muß genau so hoch sein, wie die sich im betreffenden Land im Umlauf befindende Geldmenge.

Unter Zuhilfenahme der bis jetzt hergeleiteten Zusammenhänge, läßt sich ein Gleichgewichtspfad der Weltwirtschaft wie folgt beschreiben:

Eine Preisfolge $\left\{p_{t}, P_{t}\right\}_{t \in Z}$, mit $q_{t}=Q_{t}, \forall t$, beschreibt ein Walras-Gleichgewicht der integrierten Weltwirtschaft bei vollkommener Voraussicht, wenn der Weltgütermarkt und die nationalen Geldmärkte in jeder Periode geräumt werden, also (2.14), (2.17) und (2.18) in jeder Periode erfüllt sind.

\subsubsection{Nicht stationäre Außenhandelsgleichgewichte}

In jeder Periode gibt es in der Weltwirtschaft drei Märkte. Dies sind der inländische und der ausländische Geldmarkt und der Weltgütermarkt. Aufgrund des Walras-Gesetzes ist eine Gleichgewichtsbedingung redundant (befinden sich zwei Märkte im Gleichgewicht, so ist der dritte Markt auch geräumt). Mittels 
eines Induktionsbeweises läßt sich zeigen, daß sogar eine weitere Gleichgewichtsbedingung überflüssig ist. ${ }^{1}$ Eine Preisfolge, die gewährleistet, daß der Weltgütermarkt in jeder Periode geräumt wird, beschreibt somit ein WalrasGleichgewicht der integrierten Weltwirtschaft. ${ }^{2}$ Es reicht somit aus, sich bei der Gleichgewichtsanalyse auf den Weltgütermarkt zu konzentrieren.

Da die Weltüberschußnachfragefunktionen aus einer Aggregation der nationalen Überschußnachfragefunktionen resultieren, erfüllen auch sie das WalrasGesetz:

$$
q_{t} \cdot \xi_{t}^{t}\left(q_{t}\right)+\xi_{t+1}^{t}\left(q_{t}\right) \equiv 0
$$

Eine Gleichgewichtsfolge läßt sich wie folgt herleiten: Bei einem gegebenen $q_{\text {t }}$ und dem damit einhergehenden Weltüberschußangebot $-\xi_{t}$, erhält man aus (2.19) die Weltüberschußnachfrage $\xi_{t+1}^{t}$ der Alten der folgenden Periode. Ana$\log$ erhält man aus (2.5) und (2.10) die nationalen Überschußnachfragen $z_{t+1}^{t}$ und $Z_{t+1}^{t}$. Damit der Weltgütermarkt in Periode $t+1 \mathrm{im}$ Gleichgewicht ist, muß das Weltüberschußangebot $-\xi_{t+1}^{t+1}$ der Jungen dieser Periode mit der Weltüberschußnachfrage $\xi_{t+1}^{t}$ übereinstimmen (vgl. (2.14)). Das $q_{t+1}$, welches diese Gleichheit gewährleistet, determiniert auch die nationalen Überschußangebote $-z_{t+1}^{t+1}$ und $-Z_{t+1}^{t+1}$. Der Vergleich der nationalen Überschußangebote mit den nationalen Überschußnachfragen gibt die Handelsbilanz der Periode $t+1$ an (vgl. (2.15) und (2.16)). Die nachfolgenden gleichgewichtigen intertemporalen

\footnotetext{
${ }^{1}$ Vgl. Schittko und Eckwert (1988), S. 81f.

2 Entscheidend ist, daß die Überschußnachfragen durch das intertemporale Preisverhältnis determiniert werden, nicht aber die nationalen nominalen Geldnachfragen. Für ihre Höhe sind die absoluten Güterpreise von Relevanz. Es läßt sich immer eine Konstante finden, mit der man die Güterpreisfolge multiplizieren kann, um so eine Geldmarkträumung herbeizuführen. Die Multiplikation der Preisfolge mit einer Konstanten läßt die intertemporalen Preisverhältnisse und damit die Güterallokation unberührt. Hinter der doppelten Redundanz steckt folgender ökonomischer Sachverhalt: Beide Währungen sind vollkommene Substitute im Portfolio der Anleger. Somit müssen sie gleich teuer sein, was sich in dem international übereinstimmenden intertemporalen Preisverhältnis manifestiert. Die Arbitragefreiheit bedingt, daß genau dann, wenn ein nationaler Geldmarkt geräumt ist, es der andere auch ist. Somit ist eine der Geldmarktgleichgewichtsbedingungen tatsächlich redundant. Faktisch gibt es nur zwei ökonomisch relevante Gleichgewichtsbedingungen, von denen eine gemäß dem Walras-Gesetz wiederum redundant ist.
} 
Preisverhältnisse und die mit ihnen einhergehende Allokation erhält man auf die gleiche Weise.

Da die Ökonomien der beiden Länder vom Samuelson-Typ sind, besitzen beide zwei langfristige Autarkiegleichgewichte. Dies sind das nominale Autarkiegleichgewicht und das reale Autarkiegleichgewicht. ${ }^{1}$ Sei $q_{r}$ das Preisverhältnis im realen Autarkiegleichgewicht des Inlands und $q_{R}$ das im realen Autarkiegleichgewicht des Auslands. Bei allen Preisverhältnissen, die höher als das Preisverhältnis im realen langfristigen Gleichgewicht einer Ökonomie sind, sind die Akteure des betreffenden Landes gewillt in ihrer Jugend zu sparen. Genau dann, wenn $q_{r} \neq q_{R}$, kann es zur Entstehung von Innengeld kommen. Mit Innengeld bezeichnet man durch die Akteure emittierte Schuldtitel. ${ }^{2}$

In jeder Periode leben in der Weltwirtschaft vier repräsentative Akteure. Dies sind zwei junge Akteure und zwei alte Akteure, von denen jeweils einer ein Inländer und einer ein Ausländer ist. Da die Akteure nur zwei Perioden leben, können Innengeldbeziehungen nur zwischen Akteuren der gleichen Generation entstehen. Sei nun angenommen, daß $q_{r}<q_{R}$. Bei allen $q_{t}>q_{R}$ findet nur intergenerationaler Tausch statt. Die Verzinsung der Ersparnis ist hinreichend hoch, um die inländischen und die ausländischen Akteure zum Sparen zu motivieren. Die jungen Akteure in beiden Ländern sparen, und die alten Akteure entsparen. Bei $q_{t}=q_{R}$ ist die Verzinsung der Geldhaltung nicht mehr hoch genug, um die Ausländer zum Sparen zu veranlassen. Sie konsumieren ihre Erstausstattung. Für die inländischen Akteure ist die Verzinsung der Geldhaltung allerdings noch hoch genug, es gilt ja noch immer $q_{t}>q_{r}$. Daher sind die Inländer immer noch bereit in der Jugend zu sparen und im Alter zu entsparen. Bei $q_{t}<q_{R}$ entsteht Innengeld. Jetzt findet auch intragenerationaler Handel statt. Die Verzinsung ist jetzt so gering, daß die ausländischen Akteure ein Interesse haben sich in ihrer Jugend zu verschulden (einen Kredit aufzunehmen)

\footnotetext{
' Im nominalen langfristigen Gleichgewicht findet intergenerationaler Tausch statt und Geld Verwendung. Das intertemporale Preisverhältnis ist hier eins, es herrscht also Preisstabilität. Im realen langfristigen Gleichgewicht findet kein intergenerationaler Tausch statt. Die Akteure konsumieren ihre Erstausstattung, und Geld findet keine Verwendung. Das intertemporale Preisverhältnis ist hier kleiner als eins, es herrscht also Inflation, was die Kaufkraft des Geldes gegen null konvergieren läßt. Vgl. dazu auch Wallace (1980), S. 53ff.

2 vgl. dazu z.B. Azariadis (1993), S. $175 \mathrm{ff}$.
} 
und im Alter zu sparen (den Kredit zu tilgen). Aus diesem Grund gehen sie eine Kreditbeziehung mit dem inländischen Akteur der gleichen Generation ein. Dieser hat bei allen $q_{t}>q_{r}$ ein Interesse in seiner Jugend zu sparen und im Alter zu entsparen. Beide Ökonomien sind per Annahme vom Samuelson-Typ, somit gilt $q_{R}<1$. Damit ist die Gütermenge, die der Ausländer im Alter dem Inländer der gleichen Generation zur Tilgung seines Kredites übertragen muß, geringer als die Gütermenge, die er in der Jugend für seine Schuldverschreibung erhält. ${ }^{1}$ Für diesen internationalen Handel ist kein Geld notwendig, der Handel findet zwischen Akteuren der gleichen Generation statt (intragenerationaler Handel). Wenn $q_{t} \leq q_{R}$, findet im Ausland kein intergenerationaler Tausch mehr statt (die Ausländer sparen nicht, sie entsparen) und Geld damit keine Verwendung mehr. Für den inländischen intergenerationalen Tausch, der bei $q_{t}>q_{r}$ weiterhin stattfindet, findet Geld im Inland allerdings weiterhin Verwendung.

\subsubsection{Die langfristigen Außenhandelsgleichgewichte}

Im langfristigen Gleichgewicht sind das intertemporale Preisverhältnis und die Allokation über die Zeit konstant. Die Weltwirtschaft besitzt zwei langfristige Gleichgewichte (dies folgt aus Gleichgewichtsbedingung (2.14) und Identität (2.19)). Das eine geht mit $q_{S}=1$ einher und das andere mit $q_{l}<1$, wobei $q_{r}<q_{I}<q_{R}$, da per Annahme $q_{r}<q_{R}<1$ gilt. Im mit $q_{S}$ einhergehenden Gleichgewicht herrscht Preisstabilität. Wie man aus den Identitäten (2.5) und (2.10) unmittelbar folgen kann, ist die Handelsbilanz in diesem Gleichgewicht in jeder Periode ausgeglichen. Im mit $q_{I}$ einhergehenden Gleichgewicht herrscht Inflation, und das Inland besitzt in jeder Periode ein Handelsbilanzüberschuß bzw. das Ausland ein Handelsbilanzdefizit:

\footnotetext{
${ }^{1}$ Dies resultiert aus der Tatsache, daß $q_{t}<1$ Inflation zwischen den Perioden $t$ und $t+1$ bedeutet. Das Konsumgut wird teurer, und somit verliert das Innengeld (die Schuldverschreibung) an Kaufkraft. Das Mengenverhältnis der in den zwei aufeinanderfolgenden Lebensperioden zwischen dem Inländer und dem Ausländer einer Generation getauschten Güter, entspricht dem Kehrwert des intertemporalen Preisverhältnisses.
} 


$$
H\left(q_{I}, q_{I}\right)=z_{t}^{t}\left(q_{I}\right)+z_{t}^{t-1}\left(q_{I}\right)=-\left[Z_{t}^{t}\left(q_{I}\right)+Z_{t}^{t-1}\left(q_{I}\right)\right]<0 .
$$

Bei $q_{I}$ sind die Inländer gewillt in der Jugend zu sparen, um im Alter mehr als ihre Altersausstattung konsumieren zu können. Die Ausländer wollen sich in der Jugend verschulden und sind dafür bereit in ihrem zweiten Lebensabschnitt auf einen Teil ihrer Altersausstattung zu verzichten. Aus diesem Grund gehen die Ausländer Kreditbeziehungen mit den Inländern der gleichen Generation ein. Es entsteht somit Innengeld. Da $q_{I}<1$, ist die Gütermenge, die aufgrund dieser Kreditbeziehungen vom Inland in das Ausland fließt, größer als die, die jede Periode vom Ausland in das Inland fließt. Die von den Inländern gehaltenen Schuldscheine verlieren aufgrund der Inflation an Wert. Es findet nur noch intragenerationaler Tausch statt. Die Kaufkraft des Geldes konvergiert gegen null, da das Preisniveau gegen unendlich geht (Inflation). Es findet kein intergenerationaler Tausch mehr statt.

Man erkennt, daß genau dann, wenn bei Inflation Innengeld existiert, ein langfristiges Handelsbilanzungleichgewicht auftritt. Entsteht kein Innengeld, muß die Handelsbilanz im langfristigen Gleichgewicht ausgeglichen sein. Die Intuition ist folgende: Ein Handelsbilanzdefizit bedeutet ein $A b f l u ß$ an Geld. Würde ein Land in einem langfristigen Gleichgewicht in jeder Periode eine positive Geldmenge exportieren, würde dies eine permanente Abnahme der nationalen Geldmenge implizieren. Dies ist jedoch bei begrenzter Geldversorgung und unendlichem Zeithorizont unmöglich. ${ }^{1}$

\subsubsection{Wechselkursindeterminiertheit}

Es lassen sich zwei Politikregime unterscheiden. Im Portfolio-Autarkie-Regime ist es den Akteuren eines Landes nicht erlaubt die Währung des anderen Landes

${ }^{1}$ So gehen Schittko und Eckwert (1988), bei ihrem Beweis der Nichtexistenz eines langfristigen Gleichgewichts mit einem Handelsbilanzungleichgewicht, implizit von der Nichtexistenz von Innengeld aus; vgl. Schittko und Eckwert (1988), S. 83f. Siehe auch Freeman und Murphy (1989) zur Rolle von Innengeld, im Rahmen eines stochastischen Zwei-Länder-OLGModells. 
in ihrem Portfolio zu halten (vollständige Kapitalverkehrskontrolle). Im Laissez-Faire-Regime herrscht freier Güter- und Kapitalverkehr, und es finden keine staatlichen Devisenmarktinterventionen statt (System flexibler Wechselkurse). Kareken und Wallace (1981) zeigen, daß der Wechselkurs im Laissez-FaireRegime indeterminiert ist. Das heißt, daß der mit einer gegebenen Gleichgewichtsallokation einhergehende Wechselkurs indeterminiert ist.

Sei der Wechselkurs wie folgt definiert:

$$
e_{t}:=\frac{p_{t}}{P_{t}}
$$

Es gilt $q_{t}=Q_{t}, \forall t$ (Arbitragefreiheit). Dies ist genau dann der Fall, wenn der Wechselkurs über die Zeit konstant ist. Also gilt $e_{t}=e, \forall t$.

Sei nun ein mit $\left\{p_{t}, P_{t}\right\}_{t \in Z}$ und $\{e\}_{t \in Z}$ einhergehendes Walras-Gleichgewicht der Weltwirtschaft betrachtet. Es läßt sich nun zeigen, daß sich für jeden beliebigen Wechselkurs eine Preisfolge finden läßt, die mit der selben Gleichgewichtsallokation einhergeht, wie das betrachtete Walras-Gleichgewicht. Sei $\widetilde{e}=\lambda \cdot e, \lambda>0$ ein arbiträr fixierter Wechselkurs. Definiert man $\left\{\bar{p}_{t}, \bar{P}_{t}\right\}_{t \in \mathcal{Z}}:=\left\{p_{t}, P_{t} / \lambda\right\}_{t \in \mathcal{Z}}$, so folgt daraus $\bar{e}=p_{t} \cdot \lambda / P_{t}=\widetilde{e}$. Die intertemporalen Preisverhältnisse der definierten Preisfolge unterscheiden sich nicht von denen des betrachteten WalrasGleichgewichts. Die Auslandspreise wurden mit dem Faktor $1 / \lambda$ multipliziert und der Wechselkurs mit dem Faktor $\lambda$. Die intratemporalen Terms of Trade (der Relativpreis von Importen ausgedrückt in Exporten) haben sich somit nicht geändert $\left(\bar{e} \cdot \bar{P}_{t} / \bar{p}_{t}=e \cdot P_{t} / p_{t}=1\right)$. Damit stimmen beide Gleichgewichtsallokationen überein. ${ }^{1}$

${ }^{1}$ Das Modell von Kareken und Wallace (1981) ist etwas komplexer als das hier betrachtete. Der Staat betreibt eine monetäre Transferpolitik. Anders als im hier dargestellten Modell werden die nationalen Geldmärkte zu einem Weltgeldmarkt aggregiert. Fordert man statt dessen die separate Räumung der nationalen Geldmärkte, erhält man eine Gleichgewichtsbedingung mehr. Damit lassen sich sowohl das intertemporale Preisverhältnis als auch der Relativpreis der nationalen Währungen (der Wechselkurs) determinieren. Sei hier noch auf zwei Erweiterungen des Modells von Kareken und Wallace verwiesen. Manuelli und Peck (1990) untersuchen die Wechselkursvolatilität im Fall stochastischer Ausstattungen und Arifovic (1996) das 


\subsection{Zwei-Länder-OLG-Modelle}

\section{mit Kapitalakkumulation}

In Zwei-Länder-OLG-Modellen mit Kapitalakkumulation sparen die Akteure in Form von Produktivkapital. Ausgangspunkt bildet das Modell von Diamond (1965). Im Diamond-Modell wächst die Bevölkerung mit einer konstanten Rate, und die Ersparnis einer Periode ist gleich dem sich nicht verschleißenden Kapitalstock zu Beginn der folgenden Periode. Diamond untersucht die langfristigen Auswirkungen von Staatsverschuldung und die unterschiedlichen Auswirkungen von Innenverschuldung und Außenverschuldung. ${ }^{1}$

Betrachtet sei eine aus zwei OLG-Ökonomien bestehende Weltwirtschaft. Ihre Geschichte wird durch die diskreten Perioden $t \in Z$ indiziert. Sie beginnt in $-\infty$ und reicht bis $+\infty$. Jede Periode wird eine neue Generation zwei Perioden lebender Akteure geboren. Die Akteure aufeinander folgender Generationen sind Replikationen (ihre Präferenzordnung und Arbeitskraft sind identisch). Beide Länder besitzen die gleiche Bevölkerungsgröße, und die Mitgliederzahl aufeinander folgender Generationen wächst mit der exogen vorgegebenen Rate $n>0$ :

$$
L_{t}=(1+n) \cdot L_{t-1}, \forall t
$$

$L_{t}$ ist das nationale Arbeitskräftepotential in der Periode $t$. Da die Akteure nur in ihrer Jugend arbeiten und jeder von ihnen eine Einheit Arbeitskraft unelastisch anbietet, ist $L_{t}$ auch die Anzahl der jungen Akteure eines Landes. In Periode $t$ gibt es somit weltweit $2 \cdot L_{t}$ junge Akteure und die Weltbevölkerung beträgt $2 \cdot\left(L_{t}+L_{t} /(1+n)\right)$ bzw. die Bevölkerung eines Landes $\left(L_{t}+L_{t} /(1+n)\right)$.

Es wird unterstellt, daß die Akteure vollkommene Voraussicht besitzen. Damit stimmt der von ihnen erwartete Realzins mit dem tatsächlichen Realzins über-

Wechselkursverhalten, wenn die Akteure mittels eines genetischen Algorithmus ihre Erwartungen bilden.

${ }^{\prime}$ Er betrachtet allerdings nur den Fall, daß sich der Staat im Ausland verschulden kann, nicht aber die privaten Wirtschaftssubjekte. 
ein. Es gilt also $r_{t+1}^{e}=r_{t+1}, \forall t$. Das intertemporale Nutzenmaximierungsproblem der Akteure läßt sich wie folgt beschreiben:

$$
u\left(c_{t}^{t}, c_{t+1}^{\prime}\right) \rightarrow \underset{\substack{c_{t}^{\prime} \cdot c_{t+1}^{\prime} \\ c_{1}}}{\operatorname{Max} !}
$$

unter $\operatorname{der} N B$

$$
\begin{aligned}
c_{t}^{t}+s^{t} & =w_{t} \\
c_{t+1}^{t} & =\left(1+r_{t+1}\right) \cdot s^{t}
\end{aligned}
$$

(Budgetrestriktion Jugend)

(Budgetrestriktion Alter)

(2.23) ist das Nutzenmaximierungsproblem eines repräsentativen Inländers der Generation $t$ (das Maximierungsproblem der Ausländer lautet analog). Die intertemporale Nutzenfunktion der Akteure ist streng quasikonkav, differenzierbar und streng ansteigend, unterscheidet sich aber international, also $u \neq U$. Der Akteur bietet in der Jugend seine Arbeitskraft unelastisch an und bezieht hierfür ein reales Arbeitseinkommen von $w_{t}$. Einen Teil des Arbeitseinkommens konsumiert er, den Rest spart er gegebenenfalls ( $s^{t}$ ist die reale Ersparnis). Der Alterskonsum $c_{t+1}^{t}$ ist gleich dem Wert der Ersparnis. $r_{t+1}$ ist der Zinssatz, mit dem die Ersparnis der Periode $t$ verzinst wird. Die Jugendrestriktion und die Altersrestriktion lassen sich in die intertemporale Budgetrestriktion transformieren:

$$
c_{t}^{t}+\left(1+r_{t+1}\right)^{-1} \cdot c_{t+1}^{t}=w_{t} .
$$

(2.24) ist die in Gegenwartspreisen ausgedrückte Lebensbudgetrestriktion der Akteure. Der Kehrwert des Verzinsungsfaktors (= der Diskontfaktor) läßt sich somit als Gegenwartspreis des Zukunftskonsums interpretieren.

Die zu maximierende Lagrangefunktion lautet:

$$
L=u\left(c_{t}^{\prime}, c_{t+1}^{t}\right)+\lambda \cdot\left[w_{t}-c_{t}^{t}-\left(1+r_{t+1}\right)^{-1} \cdot c_{t+1}^{t}\right]
$$

Aus den Bedingungen erster Ordnung folgt: 
$\frac{\frac{\partial u\left(c_{t}^{t}, c_{t+1}^{t}\right)}{\partial c_{t}^{t}}}{\frac{\partial u\left(c_{t}^{t}, c_{t+1}^{t}\right)}{\partial c_{t+1}^{t}}}=1+r_{t+1} \stackrel{\text { Budgetres. }}{\longrightarrow}$ optimaler Konsumvektor $\left(c_{t}^{t}, c_{t+1}^{t}\right)$.

Das optimale Konsumprogramm zeichnet sich dadurch aus, daß die Grenzrate der Substitution im Konsum mit der Verzinsung der Ersparnis (= die ökonomische Substitutionsrate) übereinstimmt.

Die verfügbare Technologie läßt sich vermöge einer linear-homogenen neoklassischen Produktionsfunktion repräsentieren. Das Nationalprodukt $X_{t}$ der Periode $t$ ist eine Funktion des Arbeitskräftepotentials $L_{t}$ und des Kapitalstocks $K_{t}$, also gilt $X_{t}=F\left(L_{t}, K_{t}\right) . K_{t}$ ist hierbei der zu Beginn der Periode $t \mathrm{zu}$ Verfügung stehende bis Periode $t-1$ akkumulierte Kapitalstock. Das Produktionsergebnis läßt sich entweder konsumieren oder investieren (es ist Konsumgut und Kapitalgut). Im letzteren Fall erhöht es den in der folgenden Periode zu Verfügung stehenden Kapitalstock. Die Produktionsfunktion $F$ läßt sich auch in Pro-Kopf-Größen ausdrücken:

$$
x_{t}=f\left(k_{t}\right) \text {, }
$$

wobei $f(0)=0, f^{\prime}\left(k_{t}\right)>0, f^{\prime \prime}\left(k_{t}\right)<0, f^{\prime}(0)=\infty$ und $f^{\prime}(\infty)=0$. $x_{t}$ ist hierbei die Pro-Kopf-Produktion in Periode $t\left(=X_{t} / L_{t}\right)$ und $k_{t}$ das Pro-KopfKapital $\left(=K_{t} / L_{t}=\right.$ die Kapitalintensität der Produktion) dieser Periode. Wird im Folgenden von Pro-Kopf-Größen gesprochen, so ist damit die Menge pro Arbeiter gemeint und nicht die Menge pro Einwohner. ${ }^{1}$

Das unelastische Arbeitsangebot der Akteure wird, bei völlig flexiblen Reallöhnen, vollständig nachgefragt (Vollbeschäftigungsgleichgewicht auf dem Arbeitsmarkt). Bei Gewinnmaximierungsverhalten folgt, aufgrund der konstanten Skalenerträge und einer angenommenen kompetitiven Marktstruktur, daß die

\footnotetext{
${ }^{1}$ Man erinnere sich, daß es in Periode $t$ in jedem Land $L_{t}$ Arbeiter aber $\left(L_{t}+L_{t} /(1+n)\right)$ Einwohner gibt, da die Akteure im Alter nicht arbeiten.
} 
Produktionsfaktoren im Gleichgewicht mit ihrem Grenzprodukt entlohnt werden:

$$
\begin{aligned}
& r_{t}=f^{\prime}\left(k_{t}\right), \\
& w_{t}=f\left(k_{t}\right)-k_{t} \cdot f^{\prime}\left(k_{t}\right) .
\end{aligned}
$$

$r_{t}$ ist der Satz, mit dem der bis Periode $t-1$ akkumulierte Kapitalstock in Periode $t$ verzinst wird. $w_{t}$ ist der Pro-Kopf-Reallohn in dieser Periode. Es wird in dem Maße Produktivkapital akkumuliert, das gewährleistet, daß das Grenzprodukt des Kapitals gleich dem Realzins ist. Der Kapitalstock unterliegt hierbei keiner Abschreibung. ${ }^{1}$ Das Produktionsergebnis fließt somit vollständig den Produktionsfaktoren zu (Euler-Theorem), es gilt also $f\left(k_{t}\right)=w_{t}+r_{t} \cdot k_{t}$. Die Produktion ist somit gewinnlos.

\subsubsection{Außenhandel}

In beiden Ländern wird dasselbe Gut produziert, welches entweder konsumiert oder investiert werden kann (das produzierte Gut ist Konsumgut und Kapitalgut). Internationaler Güterhandel ist somit gleichbedeutend mit internationaler Verschuldung und Kreditvergabe. Es wird hierbei angenommen, daß das Finanzkapital international vollständig mobil ist, also keine Handelshemmnisse vorliegen. Somit sind Eigentumsanrechte am inländischen und am ausländischen Produktivkapital vollständige Substitute im Portfolio der Anleger, und der Realzins ist in jeder Periode in beiden Ländern gleich (Arbitragefreiheit).

\footnotetext{
${ }^{1}$ Nimmt man eine positive Abschreibungsrate $\delta$ an, so wird aus (2.24) $r_{t}=f^{\prime}\left(k_{t}\right)-\delta$. Der Realzins muß gleich dem Nettogrenzprodukt des Kapitals sein. In OLG-Modellen vom Diamond-Typ wird in der Regel keine Abschreibung des Kapitals angenommen. Ausnahmen stellen Gale (1971), Bental (1985), Sibert (1990), Ruffin und Yoon (1993), Lin (1994a) und Fisher (1995) dar. Gale (1971), Bental (1985) und Fisher (1995) gehen zusätzlich von einer über die Zeit konstanten Bevölkerungsgröße aus, das heißt $n=0$.
} 
Aufgrund der internationalen Kapitalmobilität muß zwischen dem Standort und dem Eigentum an Produktivkapital, und somit zwischen dem nationalen Kapitalstock und dem Nationalvermögen an Kapital, unterschieden werden. Dies manifestiert sich innerhalb der volkswirtschaftlichen Gesamtrechnung in der Unterscheidung zwischen Inlandsproduktion und Volkseinkommen. Aufgrund der vollständigen Kapitalmobilität, welche in einem sich international nicht unterscheidenden Zinssatz resultiert, und der Tatsache, daß beide Länder über die gleiche Technologie verfügen, sind sowohl die Kapitalintensität als auch der Pro-Kopf-Reallohn in jeder Periode in beiden Ländern gleich.

Es gibt zwei Weltmärkte. Der eine ist der Weltgütermarkt und der andere der Weltkapitalmarkt. Auf dem ersten wird das Produktionsergebnis (das Konsumgut und Kapitalgut) einer Periode gehandelt, auf dem zweiten das Finanzkapital. Es wird dabei angenommen, daß existierendes Produktivkapital sowie die Arbeitsbevölkerung international nicht mobil sind. Neu produziertes zu installierendes Produktivkapital ist allerdings international mobil. Aufgrund des Walras-Gesetzes reicht es aus einen Markt zu betrachten.

Ein Walras-Gleichgewicht der Weltwirtschaft läßt sich nun wie folgt beschreiben:

Eine Folge von Zinssätzen $\left\{r_{t+1}\right\}_{t \in Z}$ und die mit ihr einhergehende Folge nationaler Konsumpläne $\left\{\left(c_{t}^{t}, c_{t+1}^{t}\right),\left(C_{t}^{t}, C_{t+1}^{t}\right)\right\}_{t \in Z}$ beschreiben ein Walras-Gleichgewicht der integrierten Weltwirtschaft bei vollkommener Voraussicht, wenn die Konsumpläne die Optimalitätsbedingung (2.26) erfüllen, die Entlohnung der Produktionsfaktoren (2.28) und (2.29) genügt und der Weltkapitalmarkt in jeder Periode geräumt wird, also

$$
\underbrace{2 \cdot w_{t}-\left(c_{t}^{t}+C_{t}^{t}\right)}_{\text {private Ersparnis }}=\underbrace{2 \cdot k_{t+1} \cdot(1+n)}_{\text {Kapitalstock }}, \forall t .
$$

(2.30) ist die in Pro-Kopf-Größen ausgedrückte Kapitalmarktgleichgewichtsbedingung. Die reale Ersparnis der Generation $t$ muß gleich dem Kapitalstock zu Beginn der Periode $t+1$ sein. 
Da eine Zwei-Länder-Welt betrachtet wird, reicht es bei der Analyse der Zahlungsbilanz aus, die Bilanz eines Landes zu betrachten. Der Überschuß eines Landes ist das Defizit des anderen Landes. Im Folgenden werden die Handelsbilanz und die Leistungsbilanz des Inlandes hergeleitet. Die Handelsbilanz entspricht der Differenz zwischen Inlandsproduktion und Absorption. Letztere setzt sich aus Konsum und Kapitalbildung (= Investition) zusammen. Die in Pro-Kopf-Größen ausgedrückte Handelsbilanz des Inlandes in Periode $t$ ist somit

$$
h_{t}=f\left(k_{t}\right)-c_{t}^{t}-\frac{c_{t}^{t-1}}{1+n}-\left[(1+n) \cdot k_{t+1}-k_{t}\right]
$$

Die Leistungsbilanz ist die Differenz zwischen dem Volkseinkommen und der inländischen Absorption. Das Volkseinkommen ist hierbei die Summe aus der Inlandsproduktion und dem aus dem bestehenden Nettoauslandsvermögen erzielten Faktoreinkommen. Sei $a_{t}$ das inländische Pro-Kopf-Finanzvermögen zu Beginn der Periode $t$. Das inländische Finanzvermögen zu Beginn der Periode $t$ ist gleich der Ersparnis der Inländer der Generation $t-1$. Somit gilt $a_{t}$ $=\left(w_{t-1}-c_{t-1}^{t-1}\right) /(1+n)$. Das Pro-Kopf-Nettoauslandsvermögen zu Beginn der Periode $t$ beträgt $a_{t}-k_{t}$ und wird auch Auslandsposition genannt. Das ProKopf-Faktoreinkommen aus der Auslandsposition beträgt $r_{t} \cdot\left(a_{t}-k_{t}\right)$. Die in Pro-Kopf-Größen ausgedrückte inländische Leistungsbilanz der Periode $t$ lautet dann:

$$
v_{t}=f\left(k_{t}\right)+r_{t} \cdot\left(a_{t}-k_{t}\right)-c_{t}^{t}-\frac{c_{t}^{t-1}}{1+n}-\left[(1+n) \cdot k_{t+1}-k_{t}\right]
$$

Die Produktionsfaktoren werden mit ihrem Grenzprodukt entlohnt und der Gewinn ist null, also $f\left(k_{t}\right)=w_{t}+r_{t} \cdot k_{t}$. Damit wird aus (2.32):

$$
v_{t}=w_{t}+r_{t} \cdot a_{t}-c_{t}^{t}-\frac{c_{t}^{t-1}}{1+n}-\left[(1+n) \cdot k_{t+1}-k_{t}\right]
$$


Ein Leistungsbilanzüberschuß läßt sich auch als Nettoauslandsinvestition interpretieren. Die Nettoauslandsinvestition ist die positive Differenz zwischen inländischer Vermögensakkumulation und Kapitalbildung, also

$$
v_{t}=\left[(1+n) \cdot a_{t+1}-a_{t}\right]-\left[(1+n) \cdot k_{t+1}-k_{t}\right]
$$

\subsubsection{Das langfristige AuBenhandelsgleichgewicht}

Im langfristigen Gleichgewicht sind die Pro-Kopf-Größen und der Zinssatz über die Zeit konstant. Das langfristige Freihandelsgleichgewicht der Weltwirtschaft läßt sich wie folgt beschreiben:

$$
\begin{aligned}
& 2 \cdot w-\left(c_{t}^{t}-C_{t}^{t}\right)=2 \cdot k \cdot(1+n), \\
& a=\frac{w-c_{t}^{t}}{1+n}, \\
& h=f(k)-c_{t}^{t}-\frac{c_{t}^{t-1}}{1+n}-n \cdot k=(n-r) \cdot(a-k), \\
& v=f(k)+r \cdot(a-k)-c_{t}^{t}-\frac{c_{t}^{t-1}}{1+n}-n \cdot k=n \cdot(a-k) .
\end{aligned}
$$

Größen, die über die Zeit konstant sind werden, mit Ausnahme der individuellen Konsummengen, ohne Zeitindex geschrieben. (2.35) ist das stationäre Kapitalmarktgleichgewicht und (2.36) gibt das langfristige Pro-Kopf-Finanzvermögen der Inländer an. (2.37) ist die langfristige inländische Handelsbilanz und (2.38) die Leistungsbilanz. Das zweite Gleichheitszeichen in (2.37) und (2.38) erhält man unter Verwendung der intertemporalen Budgetrestriktion (2.24) und der Tatsache, daß die Konsumpläne über die Zeit konstant sind, Gleichung (2.36) und der Tatsache, daß $f(k)=w+r \cdot k$. Die Bedingungen für $A, H$ und $V$ lauten analog. 
Aus (2.37) und (2.38) erkennt man, daß eine langfristig ausgeglichene Handelsund Leistungsbilanz keine notwendige Gleichgewichtsbedingung ist. Ist ein Land langfristig ein Gläubigerland $(a>k)$, so erfordert die Aufrechterhaltung eines konstanten Pro-Kopf-Auslandsvermögens einen langfristigen Leistungsbilanzüberschuß. Ist ein Land langfristig ein Schuldnerland $(a<k)$, so besitzt es ein langfristiges Leistungsbilanzdefizit. Die Tatsache, daß die Leistungsbilanz eines Landes, bei einer langfristig nicht ausgeglichenen Pro-Kopf-Auslandsposition, ein permanentes Ungleichgewicht aufweist, resultiert aus der positiven Wachstumsrate der Bevölkerung.

Zur Bestimmung des Vorzeichens der Handelsbilanz ist eine Fallunterscheidung zu treffen. Die Kapitalintensität entspricht der goldenen Regel, wenn das Grenzprodukt des Kapitals gleich der Wachstumsrate der Bevölkerung ist, also $f^{\prime}\left(k_{\text {Gold }}\right)=n .{ }^{1}$ Die Weltwirtschaft befindet sich in einem dynamisch effizienten Gleichgewicht, wenn die Kapitalintensität nicht höher als $k_{\text {Gold }}$ ist, wenn also $r \geq n$ gilt. Sie befindet sich in einem dynamisch ineffizienten Gleichgewicht, wenn die Kapitalintensität höher als $k_{\text {Godd }}$ ist (Überkapitalisierung), wenn also $r<n$ gilt. $^{2}$

' Vgl. z.B. Barro und Sala-i-Martin (1995), S. 19ff. Die goldene Regel beschreibt die, bei gegebener Technologie, maximalen Konsummöglichkeiten einer Ökonomie, die sie langfristig besitzt, unter der Voraussetzung, daß die von der goldenen Regel vorgeschriebene Kapitalintensität einmal erreicht ist. Unterliegt der Kapitalstock einer positiven Abschreibungsrate $\delta$, so gilt $f^{\prime}\left(k_{\text {Gold }}\right)=n+\delta$. Die goldene Regel der Kapitalakkumulation stimmt mit Samuelsons Konzept der sozialen Optimalität überein. Gemäß Samuelsons biologischer Theorie des Zinses ist eine Allokation genau dann optimal, wenn der mit ihr einhergehende Zinssatz gleich der Wachstumsrate der Bevölkerung ist; vgl. Samuelson (1958), S. 471ff. Im sozialen Optimum gilt $f^{\prime}\left(k_{\text {Gold }}\right)=r=n$. Zu diesem Themenkomplex, siehe auch Starrett (1972), S. $281 \mathrm{ff}$. ${ }^{2}$ Beide Fälle sind möglich. Der Grund, aus dem es zur Überkapitalisierung kommen kann, ist der, daß der Planungshorizont der Akteure endlich (genau zwei Perioden) und jener der Ökonomie unendlich ist. Siehe dazu z.B. Romer (1996), S. 81 ff. Der Einkommensvektor der Akteure ist extrem linksschief, da sie nur in ihrer Jugend arbeiten und ein Arbeitseinkommen beziehen. Das Arbeitseinkommen nimmt über den individuellen Lebenszyklus drastisch ab, was die Akteure zu verstärktem Sparen motiviert. Makroökonomisch betrachtet ist der Einkommensstrom intertemporal ausgeglichener, da es in jeder Periode junge Akteure gibt, die arbeiten. Der gesellschaftlich optimale Sparprozeß differiert somit vom individuell optimalen, was zu einer Kapitalakkumulation führen kann, die das gesellschaftlich optimale Niveau überschreitet. 
Wie man aus (2.37) erkennt, besitzt ein Gläubigerland $(a>k)$ im langfristigen Gleichgewicht entweder ein Handelsbilanzdefizit (Fall $r>n$ ), einen Handelsbilanzüberschuß (Fall $r<n$ ) oder eine ausgeglichene Handelsbilanz (Fall $r=n$ ). Für ein Schuldnerland $(a<k)$ gilt das genau Umgekehrte. Daß die Handelsbilanz eines Gläubigerlandes einen Überschuß aufweisen kann, was ein ungewöhnliches Ergebnis ist, hängt mit der positiven Wachstumsrate der Bevölkerung zusammen. Ist die Wachstumsrate höher als der Zinssatz, sind die Auslandsinvestitionen, die zur Aufrechterhaltung eines konstanten Pro-Kopf-Auslandsvermögens notwendig sind, höher als die Faktorerträge aus dem Ausland, was in einem Handelsbilanzüberschuß resultiert. ${ }^{1}$

Da das Arbeitseinkommen und die Verzinsung der Ersparnis in beiden Ländern gleich sind, trifft dies auch für das Lebenseinkommen zu. Die Budgetmengen der Akteure in den beiden Ländern sind gleich. Es kommt genau dann zu internationalen Kreditbeziehungen, wenn sich die nationalen Konsumpläne unterscheiden, wenn also $\left(c_{t}^{t}, c_{t+1}^{t}\right) \neq\left(C_{t}^{t}, C_{t+1}^{t}\right)$ gilt. Eine notwendige Bedingung hierfür ist $u \neq U$. Eine Gläubiger- oder Schuldnerposition bedeutet keine Veränderung der Konsummöglichkeiten. Der Gegenwartswert des Lebenseinkommens (das Arbeitseinkommen) bleibt hiervon unberührt und ist gleich dem Gegenwartswert des Lebenskonsums (vgl. (2.24)). Internationaler Handel und damit internationale Verschuldung und Kreditvergabe ist hier Ergebnis des Wunsches den Konsumpfad vom Einkommenspfad zu separieren, um so das Lebenskonsumprofil gemäß den individuellen Präferenzen optimal zu gestalten.

Buiter (1981) betrachtet den Fall, daß sich die Länder nur bezüglich der Zeitpräferenz der sie bevölkernden Akteure unterscheiden. Akteure mit einer höheren Zeitpräferenz sind ungeduldiger als Akteure mit einer niedrigeren Zeitpräfe-

${ }^{1}$ In diesem Zusammenhang sei hier noch auf Gale (1971) verwiesen. Im Modell von Gale wird neben einer konstanten Bevölkerungsgröße $(n=0)$ ein sich nach einer Periode vollständig abschreibender Kapitalstock unterstellt $(\delta=1)$. Liegt die als konstant angenommene Sparquote der Akteure (der Anteil am Arbeitseinkommen, der gespart wird) zwischen den nationalen Investitionsquoten (der Anteil am Produktionsergebnis, der investiert wird), so existiert neben dem langfristigen Gleichgewicht mit ausgeglichener Handelsbilanz ein weiteres langfristiges Gleichgewicht mit einem Handelsbilanzungleichgewicht; vgl. Gale (1971), S. 144f. Ein langfristiges Handelsbilanzungleichgewicht ist auch in diesem Modell die Regel und nicht die Ausnahme. 
renz. Eine höhere Zeitpräferenz bedeutet, daß der in der Zukunft erreichbare Nutzen stärker abdiskontiert wird. Sein Gegenwartswert ist somit geringer. Nimmt man an, daß die intertemporale Nutzenfunktion der Akteure additiv separabel ist, so läßt sich diese Eigenschaft wie folgt formalisieren:

$$
u\left(c_{t}^{t}, c_{t+1}^{t}\right)=v\left(c_{t}^{t}\right)+(1+\rho)^{-1} \cdot v\left(c_{t+1}^{t}\right)
$$

$\rho$ ist hier die Zeitpräferenzrate der Akteure. Die Zeitpräferenzrate ist somit gleich der Grenzrate der Substitution im Konsum, bei gleichen Konsummengen in der Jugend und im Alter, minus eins. ${ }^{1}$ Anders ausgedrückt, besitzen Akteure mit einer höheren Zeitpräferenzrate eine geringere Sparneigung. Sie wollen also eher in der Jugend als im Alter konsumieren. Nimmt man nun an, daß die Ausländer eine höhere Zeitpräferenz als die Inländer besitzen, die Ausländer also ungeduldiger als die Inländer sind, äußert sich das in $\rho<\mathrm{P}$.

Buiter zeigt, daß die Leistungsbilanz des Landes mit den ungeduldigeren Akteuren ein Defizit aufweist. ${ }^{2}$ In diesem Land ist der Jugendkonsum höher als im anderen Land, denn die Akteure sparen einen geringeren Teil ihres Arbeitseinkommens. Dies resultiert in einer negativen Auslandsposition (die Akteure des anderen Landes halten einen Teil des nationalen Kapitalstocks), da die Kapitalintensität und das Arbeitseinkommen in beiden Ländern gleich ist. Bei konstanter Pro-Kopf-Außenverschuldung und wachsender Bevölkerung bedingt dies ein langfristiges Leistungsbilanzdefizit (vgl. (2.38)).

Schmid und Großmann (1986) untersuchen die Auswirkungen einer Veränderung der Zeitpräferenzrate. ${ }^{3}$ Eine Reduktion der Zeitpräferenzrate, sei es in einem oder in beiden Ländern, führt zu einer Vergrößerung des Weltkapitalstocks bzw. der Länderkapitalstöcke, einer Reduktion des Zinssatzes und damit zu einer Verbesserung der Einkommensverteilung zugunsten der arbeitenden jungen Generation. Dieser Zusammenhang ist aus (2.28) und (2.29) leicht ersichtlich.

\footnotetext{
${ }^{1}$ Eine schöne Diskussion des das intertemporale Verhalten determinierenden Parameters Zeitpräferenzrate findet sich in Neumann (1990), Kap. 4.

${ }^{2}$ Vgl. Buiter (1981), S.784ff.

${ }^{3}$ Vgl. Schmid und Großmann, S. 44ff.
} 
Die Kapitalintensität läßt sich als Funktion des Lohnsatzes ausdrücken, also $k_{t}\left(w_{t}\right)$ und $k_{t}^{\prime}\left(w_{t}\right)=-1 / k_{t}\left(w_{t}\right) \cdot f^{\prime \prime}\left[k_{t}\left(w_{t}\right)\right]>0$. Damit folgt:

$$
r_{t}^{\prime}\left(w_{t}\right)=f^{\prime \prime}\left[k_{t}\left(w_{t}\right)\right] \cdot k_{t}^{\prime}\left(w_{t}\right)=-\frac{1}{k_{t}\left(w_{t}\right)}<0
$$

(2.40) wird Faktor-Preis-Grenze genannt. Eine Erhöhung (Reduktion) des Reallohnes führt zu einer Reduktion (Erhöhung) des Realzinses.

Eine Veränderung der relativen Zeitpräferenz in den beiden Ländern wirkt sich entscheidend auf den Leistungsbilanzsaldo und die Auslandsposition aus. Eine Reduktion der Zeitpräferenzrate der Akteure verbessert in der Regel die Auslandsposition des betreffenden Landes. Im Fall eines Schuldnerlandes ist es jedoch möglich, daß sich die Pro-Kopf-Außenverschuldung erhöht und nicht reduziert. Dieser Ausnahmefall tritt dann auf, wenn die nationalen Zeitpräferenzraten hinreichend unterschiedlich sind: Die Reduktion der Zeitpräferenzrate geht mit einer Erhöhung der Kapitalintensität und einer Reduktion des Zinssatzes einher. Die Akteure des Schuldnerlandes sparen einen geringeren Teil ihres Arbeitseinkommens als die Akteure des Gläubigerlandes. Die erhöhte Kapitalintensität bedingt ein erhöhtes Arbeitseinkommen. Es ist nun möglich, daß die Steigerung der Ersparnis der jungen Akteure im Gläubigerland höher ist als im Schuldnerland, was sich in einer Verschlechterung der Auslandsposition des Schuldnerlandes manifestiert.

Ruffin und John (1993) untersuchen die langfristigen Auswirkungen der Kapitalmobilität auf die Vermögensakkumulation und die Wohlfahrt der Akteure. ${ }^{1}$ Die Ersparnis der Akteure ist eine Funktion des Zinssatzes und ihres Arbeitseinkommens, also $s(r, w)$. Somit gilt:

$$
\frac{d s}{d r}=\frac{\partial s}{\partial r}+\frac{\partial s}{\partial w} \cdot \frac{d w}{d r}
$$

Gemäß (2.40) gilt entlang der Faktor-Preis-Grenze $d w / d r=-k$. Damit folgt:

\footnotetext{
' Vgl. Ruffin und John (1993), S. $131 \mathrm{ff}$.
} 


$$
\left.\frac{d s}{d r}\right|_{d w=-k \cdot d r}=\underbrace{\frac{\partial s}{\partial r}}_{?}-k \cdot \underbrace{\frac{\partial s}{\partial w}}_{>0} .
$$

Bei Normalgütern steigt die Ersparnis mit dem Arbeitseinkommen. Aufgrund des in der Jugend gegenläufigen Einkommens- und intertemporalen Substitutionseffekts erhöht oder reduziert eine Erhöhung des Zinssatzes die Ersparnis. Somit ist das Vorzeichen von (2.42) indeterminiert. (2.42) gibt die Veränderung der Sparrate an, als Reaktion auf eine Veränderung des Zinssatzes, unter Berücksichtigung der durch die Faktor-Preis-Grenze gegebenen Interdependenz zwischen dem Realzins und dem Reallohn.

Es besteht eine positive Beziehung zwischen den Vermögen in den beiden Ländern, das heißt, je höher der Vermögensbestand in einem Land ist, desto höher ist der langfristige Vermögensbestand im anderen Land. Anders ausgedrückt profitiert das ärmere Land vom Reichtum des reicheren Landes. Der Grund ist die vollständige Kapitalmobilität: Sei angenommen, daß beide Länder unterschiedlich vermögend sind. Da das Kapital vollständig mobil ist, fließt es aus dem reicheren Land in das ärmere Land, da es dort rentabler investiert werden kann. Gemäß der Faktor-Preis-Grenze reduziert sich der Zinssatz und erhöht sich der Lohnsatz im ärmeren Land. Die Erhöhung des Arbeitseinkommens wirkt in Richtung einer Erhöhung der Ersparnis und damit wächst der Vermögensbestand. Der Einfluß der Vermögensakkumulation in einem Land auf die Vermögensakkumulation im anderen Land ist somit unabhängig von der Auslandsposition.

Ist der Zinseffekt auf die Sparrate negativ, die Ableitung (2.42) also kleiner als null, so geht mit einer Erhöhung des Zinssatzes eine Reduktion der Vermögensakkumulation einher. In diesem Fall erhöht der Übergang von Autarkie zu Freihandel (= vollständige Kapitalmobilität) den langfristigen Kapitalstock des Kapital importierenden Landes und reduziert den langfristigen Kapitalstock des Kapital exportierenden Landes. Zur Erklärung sei der Fall betrachtet, daß das Vermögen der Inländer im langfristigen Autarkiegleichgewicht größer ist als das der Ausländer: Nach einer Öffnung zum Weltmarkt gleicht sich die Kapitalintensität international an. Der ausländische Kapitalstock vergrößert sich, und 
der inländische Kapitalstock verkleinert sich. Im Zuge dieses Prozesses tätigen die Inländer Auslandsinvestitionen. Sie sind Kapitalexporteure und die Ausländer Kapitalimporteure. Als Folge der vollständigen Kapitalmobilität reduziert sich die Vermögensakkumulation der Inländer und die der Ausländer erhöht sich, da der Zinssatz aus Sicht der Inländer gestiegen ist und aus Sicht der Ausländer gesunken. Gegenüber der Autarkiesituation hat sich das Pro-KopfFinanzvermögen der Inländer somit reduziert und das Pro-Kopf-Finanzvermögen der Ausländer erhöht. Die Auslandsposition der Inländer ist allerdings positiv und die der Ausländer negativ.

Bezüglich der Auswirkung der Kapitalmobilität auf die Wohlfahrt der Akteure läßt sich folgendes sagen: Die gegenwärtige Generation profitiert von Kapitalexporten und wird durch Kapitalimporte schlechter gestellt. Durch den Kapitalexport kann die Ersparnis gewinnbringender investiert werden, da das Kapital im Ausland höher verzinst wird. Im Kapital importierenden Land reduziert sich, aufgrund der Erhöhung der Kapitalintensität, die Verzinsung des Kapitals, was sich negativ auf die Rendite der Ersparnis auswirkt. Für die zukünftigen Generationen gilt das Umgekehrte. Diese profitieren von Kapitalimporten und werden durch Kapitalexporte tendenziell schlechter gestellt, da eine höhere Kapitalintensität das Nationalprodukt vergrößert. Als Folge würde es ein Akteur eines potentiell Kapital exportierenden Landes präferieren, unter Autarkie geboren zu werden, als im langfristigen Freihandelsgleichgewicht. Einmal geboren, würde er allerdings für eine Öffnung zum Weltmarkt votieren. Ein Akteur eines potentiell Kapital importierenden Landes würde es präferieren, im langfristigen Freihandelsgleichgewicht geboren zu werden. ${ }^{1}$

\footnotetext{
${ }^{1}$ In diesem Zusammenhang sei hier auch auf Bental (1985) verwiesen. Im Modell von Bental wird eine Zwei-Sektoren-Technologie angenommen. Im einen Sektor wird das Konsumgut produziert und im anderen das Kapitalgut, und es besteht eine Heckscher-Ohlin-Außenhandelsstruktur. Bental stellt nun einen Wohlfahrtsvergleich zwischen zwei Politikregimen an. Im Laissez-Faire-Regime werden das Kapitalgut und das Konsumgut international frei gehandelt, im Portfolio-Autarkie-Regime ist nur das Konsumgut international mobil. Im Laissez-FaireRegime ist die Verzinsung des Kapitals international gleich, im Portfolio-Autarkie-Regime kann es zu einer Spezialisierung in der Produktion kommen. Obwohl der Übergang vom Portfolio-Autarkie- zum Laissez-Faire-Regime zu einer effizienteren Ressourcenallokation fuihrt, muß dies keine Pareto-Verbesserung darstellen, und es existiert kein temporaler oder intertemporaler Umverteilungsmechanismus, der eine solche herbeiführen kann.
} 


\subsubsection{Nicht stationäre Außenhandelsgleichgewichte}

Befindet sich die Weltwirtschaft in einem nicht stationären Gleichgewicht, so verändern sich die Pro-Kopf-Größen und der Zinssatz im Zeitablauf. Mit der Erkenntnis, daß $a_{t}=\left(w_{t-1}-c_{t-1}^{t-1}\right) /(1+n)$, läßt sich die inländische Leistungsbilanz (2.32) wie folgt umschreiben:

$v_{t}=f\left(k_{t}\right)+r_{t} \cdot\left(\frac{w_{t-1}-c_{t-1}^{t-1}}{1+n}-k_{t}\right)-c_{t}^{t}-\frac{c_{t}^{t-1}}{1+n}-\left[(1+n) \cdot k_{t+1}-k_{t}\right]$

Korrespondierend dazu lautet die ausländische Leistungsbilanz:

$V_{t}=f\left(k_{t}\right)+r_{t} \cdot\left(\frac{w_{t-1}-C_{t-1}^{t-1}}{1+n}-k_{t}\right)-C_{t}^{t}-\frac{C_{t}^{t-1}}{1+n}-\left[(1+n) \cdot k_{t+1}-k_{t}\right]$

In einer aus zwei Ländern bestehenden Weltwirtschaft gilt natürlich $v_{t}+V_{t} \equiv 0$. Es stellt sich nun die Frage, ob die Leistungsbilanz eines Landes, dessen Akteure eine niedrigere Zeitpräferenz als die Akteure des anderen Landes besitzen, auch im nicht stationären Gleichgewicht einen Überschuß aufweist, wie dies der Fall im langfristigen Gleichgewicht ist.

Sei wieder der Fall betrachtet, daß die Inländer geduldiger als die Ausländer sind, also $\rho<\mathrm{P}$ (vgl. (2.39)). Die niedrigere Zeitpräferenz der Inländer bedingt, $\mathrm{da} ß$ ihr Jugendkonsum niedriger und ihr Alterskonsum höher als jener der Ausländer ist. Es gilt also $c_{t-1}^{t-1}<C_{t-1}^{t-1}, c_{t}^{t}<C_{t}^{t}$ und $c_{t}^{t-1}>C_{t}^{t-1}$. Der niedrigere Jugendkonsum wirkt in Richtung eines inländischen Leistungsbilanzüberschusses, der höhere Alterskonsum in Richtung eines Defizits. Aus (2.43) und (2.44) folgt, daß die inländische Leistungsbilanz genau dann einen Überschuß aufweist, wenn

$$
r_{t} \cdot\left(\frac{c_{t-1}^{t-1}-C_{t-1}^{t-1}}{1+n}\right)+c_{t}^{t}-C_{t}^{t}<\frac{C_{t}^{t-1}-c_{t}^{t-1}}{1+n}
$$


Unter Verwendung der intertemporalen Budgetrestriktion der Akteure wird daraus:

$$
c_{t}^{\prime}-C_{t}^{t}<\frac{c_{t-1}^{t-1}-C_{t-1}^{t-1}}{1+n}
$$

Wegen $n>0$ und der Tatsache, daß die Konsumpläne im langfristigen Gleichgewicht über die Zeit konstant sind, ist Bedingung (2.46) dort auf jeden Fall erfüllt $\left(c_{t-1}^{t-1}=c_{t}^{t}<C_{t-1}^{t-1}=C_{t}^{t}\right)$. Um die Gültigkeit im nicht stationären Gleichgewicht zu zeigen, verwendet man die Eigenschaft $L_{t}=(1+n) \cdot L_{t-1}$. Aus (2.46) wird so:

$$
L_{t} \cdot c_{t}^{t}-L_{t-1} \cdot c_{t-1}^{t-1}<L_{t} \cdot C_{t}^{t}-L_{t-1} \cdot C_{t-1}^{t-1}
$$

Die Leistungsbilanz des Inlands weist in Periode $t$ somit genau dann einen Überschuß auf, wenn die zwischen den Perioden $t-1$ und $t$ stattfindende Steigerung des aggregierten inländischen Jugendkonsums geringer als die Steigerung des aggregierten ausländischen Jugendkonsums ist.

Die Leistungsbilanz eines Landes ist die Differenz zwischen der nationalen Ersparnis und der nationalen Kapitalbildung. Aufgrund der vollständigen Kapitalmobilität ist die Kapitalintensität und damit die Kapitalbildung, da die $\mathrm{Be}$ völkerung in beiden Ländern gleich groß ist und mit der gleichen Rate wächst, in jeder Periode in beiden Ländern gleich. Somit läßt sich Bedingung (2.47) äquivalent schreiben als

$$
L_{t} \cdot\left(w_{t}-c_{t}^{t}\right)-L_{t-1} \cdot\left(w_{t-1}-c_{t-1}^{t-1}\right)>L_{t} \cdot\left(w_{t}-C_{t}^{t}\right)-L_{t-1} \cdot\left(w_{t-1}-C_{t-1}^{t-1}\right)
$$

Die Leistungsbilanz des Inlands weist in Periode $t$ somit genau dann einen Überschuß auf, wenn die inländische Ersparnis in dieser Periode höher als die ausländische Ersparnis ist. Aufgrund der geringeren Zeitpräferenzrate der Inländer, sparen diese in jeder Generation mehr als die Ausländer der korrespondierenden Generation. Dies muß jedoch nicht für die nationale Ersparnis einer Periode gelten. Diese setzt sich aus Sparen der Jungen und Entsparen der Alten zusammen. Zwar sparen die Jungen Inländer mehr als die jungen Ausländer, die 
alten Inländer entsparen aber auch mehr als die alten Ausländer. $\rho<\mathrm{P}$ garantiert also nicht die Gültigkeit von (2.48). Die Leistungsbilanz eines Landes, dessen Akteure relativ geduldig sind, kann somit im nicht stationären Gleichgewicht ein Defizit aufweisen.

\subsubsection{Fiskalpolitische Aspekte}

Bis jetzt wurde der öffentliche Sektor vollständig vernachlässigt. Der Staat kann auf verschiedenartigste Weise in den Wirtschaftskreislauf eingreifen. Auf der Einnahmenseite kann er sich verschulden, indem er Staatsanleihen emittiert, oder er kann die Akteure auf unterschiedliche Art besteuern. Er kann eine Steuer auf das Arbeitseinkommen, die Kapitaleinkünfte und den Konsum erheben. Auf der Ausgabenseite kann er den Akteuren einen Transfer zukommen lassen, oder er kann selbst Güter verbrauchen (= Staatskonsum).

\subsubsection{Die Auswirkungen von Staatsverschuldung}

Um den Einfluß des Staates auf die in diesem Abschnitt betrachtete Weltwirtschaft darzustellen, sei das Modell von Persson (1985) betrachtet. Persson untersucht die Auswirkungen von Staatsverschuldung auf den Zinssatz, das Arbeitseinkommen und die Wohlfahrt der Akteure.

Der Staat verbraucht keine Güter. Sein einziger aktiver Eingriff in den Wirtschaftskreislauf besteht in der Besteuerung des Arbeitseinkommens der Akteure und der Emission von Staatsanleihen. Die Pauschalsteuer auf das Arbeitseinkommen eines Akteurs der Generation $t$ werde mit $\tau_{t}$ bezeichnet. Sei das verfügbare Realeinkommen mit $\widetilde{w}_{t}:=w_{t}-\tau_{t}$ definiert. Die Lebensbudgetrestriktion (2.24) der Akteure wird damit zu

$$
c_{t}^{t}+\left(1+r_{t+1}\right)^{-1} \cdot c_{t+1}^{t}=\widetilde{w}_{t}
$$


Die nationalen Regierungen können nur das Arbeitseinkommen ihrer Bürger besteuern und nicht das Arbeitseinkommen der Bürger des anderen Landes. Das verfügbare Realeinkommen eines ausländischen Akteurs ist somit $\widetilde{W}_{t}=w_{t}-\mathrm{T}_{t}$. Zwar stimmt der Bruttolohn in beiden Ländern in jeder Periode weiterhin überein, (2.29) hat immer noch Gültigkeit, der Nettolohn, also das verfügbare Einkommen, kann sich aber international unterscheiden. Die Optimalitätsbedingung (2.26) bleibt von der Pauschalsteuer unberührt.

Die einzigen Staatsausgaben bestehen im Zinsendienst seiner Schulden. Reichen die Steuereinnahmen zur Finanzierung nicht aus, so wird das Budgetdefizit durch die Erhöhung des Bestandes der sich im Umlauf befindenden Staatsanleihen (= Nettoneuverschuldung) gedeckt. Die Staatsanleihen besitzen eine Periode Laufzeit. Eine in Periode $t-1$ erworbene Anleihe wird in Periode $t$ mit $\left(1+r_{t}\right)$ verzinst. Aufgrund der einperiodigen Laufzeit unterliegt der Wert der Anleihen keinen Kursschwankungen. Halter der Anleihen beziehen die sichere Rendite $r_{t}$. Auf dem Kapitalmarkt konkurrieren nun zwei Anlageinstrumente. Das eine Anlagemedium ist das Produktivkapital und das andere die Staatsanleihen. Beide sind risikolos, sind also vollkommene Substitute im Portfolio der Anleger und müssen somit bei Kapitalmarkträumung die gleiche Rendite aufweisen (Arbitragefreiheit). Tatsächlich ist $r_{t}$ der durch das Grenzprodukt des Kapitals determinierte Realzins (vgl. (2.28)), der sich weiterhin international nicht unterscheidet.

Sei $g$, die nationale reale Pro-Kopf-Staatsverschuldung zu Beginn der Periode $t$. Dann läßt sich die in Pro-Kop-Größen formulierte staatliche Budgetrestriktion der Periode $t$ wie folgt schreiben:

$$
\left(1+r_{t}\right) \cdot g_{t}=\tau_{t}+(1+n) \cdot g_{t+1} \text {. }
$$

Auf der linken Seite stehen die Staatsausgaben. Der Schuldendienst setzt sich aus dem Zinsendienst und, wegen der einperiodigen Laufzeit der Anleihen, der Schuldentilgung zusammen. Finanziert werden sie durch die Lohnsteuer und die Emission neuer Anleihen. Die Pro-Kopf-Nettoneuverschuldung ist $(1+n) \cdot g_{t+1}$ $-g_{t}$ (= staatliches Budgetdefizit der Periode $t$ ). Die Pro-Kopf-Staatsverschuldung ist über die Zeit genau dann konstant, wenn $\tau_{t}=\left(r_{t}-n\right) \cdot g_{t}$ gilt. Um 
Gleichgewichte mit negativer Steuer (= Transfer) auszuschließen wird angenommen, daß das langfristige Gleichgewicht der Weltwirtschaft dynamisch effizient ist, also $r \geq n$ gilt. $^{1}$

Für die Gleichgewichtsanalyse reicht es weiterhin aus, nur den Weltkapitalmarkt zu betrachten (Walras-Gesetz). Die Kapitalmarktgleichgewichtsbedingung (2.30) wird zu:

$$
\underbrace{\widetilde{w}_{t}+\widetilde{W}_{t}-\left(c_{t}^{t}+C_{t}^{t}\right)}_{\text {private Ersparnis }}-\underbrace{\left(g_{t+1}+G_{t+1}\right) \cdot(1+n)}_{\text {offentliche Verschuldung }}=\underbrace{2 \cdot k_{t+1} \cdot(1+n)}_{\text {Kapitalstock }} .
$$

Die Differenz zwischen der Ersparnis der Generation $t$ und der weltweiten Staatsverschuldung dieser Periode ist gleich dem Kapitalstock zu Beginn der Periode $t+1$. Staatsanleihen und Produktivkapital sind vollkommene Substitute im Portfolio der Anleger und konkurrieren um ihre Gunst. Wie man erkennt, verdrängt die Staatsverschuldung die Kapitalbildung (crowding-out).

Ein Leistungsbilanzüberschuß läßt sich gemäß (2.34) als Nettoauslandsinvestition interpretieren. Somit gilt:

$$
v_{t}=\left[(1+n) \cdot a_{t+1}-a_{t}\right]-\left\{\left[(1+n) \cdot k_{t+1}-k_{t}\right]+\left[(1+n) \cdot g_{t+1}-g_{t}\right]\right\} \text {. }
$$

Durch den Vergleich von (2.52) mit (2.34) erkennt man, daß eine positive staatliche Nettoneuverschuldung die Nettoauslandsinvestitionen und damit den Leistungsbilanzüberschuß reduziert.

Sei die reale Pro-Kopf-Auslandsposition zu Beginn der Periode $t$ mit $e_{t}$ definiert, das heißt

$$
e_{t}:=a_{t}-\left(k_{t}+g_{t}\right)
$$

\footnotetext{
${ }^{1}$ Interessant ist der Fall eines der goldenen Regel genügenden langfristigen Gleichgewichts $(r=n)$. Hier unterliegt das Arbeitseinkommen der Akteure keiner Besteuerung $(\tau=0)$. Das Wachstum der Bevölkerung reicht gerade aus, durch die Neuemission von Staatsanleihen, bei konstanter Pro-Kopf-Staatsverschuldung, den Schuldendienst zu finanzieren.
} 
In einer aus zwei Ländern bestehenden Weltwirtschaft gilt natürlich $e_{t}+E_{t} \equiv 0$. Aus (2.52) wird damit:

$$
v_{t}=(1+n) \cdot e_{t+1}-e_{t}
$$

Die Differenz zwischen der Leistungsbilanz und der Handelsbilanz ist gleich dem Ertrag aus dem Auslandsvermögen. Daraus folgt:

$$
h_{t}=v_{t}-r_{t} \cdot e_{t}=(1+n) \cdot e_{t+1}-\left(1+r_{t}\right) \cdot e_{t} \text {. }
$$

Ein langfristiges Gleichgewicht der Weltwirtschaft mit positiver Staatsverschuldung läßt sich dann wie folgt beschreiben:

$$
\begin{aligned}
& {\left[(\widetilde{w}+\widetilde{W})-\left(c_{t}^{t}+C_{t}^{t}\right)\right]-[(g+G) \cdot(1+n)]=2 \cdot k \cdot(1+n),} \\
& a=\frac{\widetilde{w}-c_{t}^{t}}{1+n}, e=a-(k+g), \quad g=\frac{-\tau}{n-r}, \\
& h=(n-r) \cdot e, \\
& v=n \cdot e .
\end{aligned}
$$

Das Kapitalmarktgleichgewicht (2.56) erhält man aus (2.51) und die Pro-KopfStaatsverschuldung aus (2.50). ${ }^{1}$ Die Handelsbilanz (2.58) erhält man aus (2.55) und die Leistungsbilanz (2.59) aus (2.54). Die Bedingungen für $A, E, G, H$ und $V$ lauten analog.

\footnotetext{
'Man erkennt, daß im ausgeschlossenen Fall $r<n$ ein überraschender Zusammenhang besteht. Hier ist eine positive Staatsschuld mit einer langfristigen Transferpolitik verbunden. Die Nettoneuverschuldung, die bei Aufrechterhaltung der konstanten Pro-Kopf-Staatsverschuldung stattfindet, reicht zur Finanzierung des Zinsendienstes aus, und es bleibt noch ein Überschuß übrig, der an die jungen Akteure transferiert werden kann.
} 
Persson (1985) untersucht die internationalen Auswirkungen einer Erhöhung der öffentlichen Verschuldung in einem Land. ${ }^{1}$ Sei angenommen, die inländische Regierung läßt den jungen Inländern der Generation $t$ einen durch Schulden finanzierten Transfer zukommen (war die Staatsverschuldung in Periode $t-1$ positiv, handelt es sich streng genommen um keinen Transfer im eigentlichen Sinn, sondern um eine Reduktion der Steuerbelastung). Gemäß der staatlichen Budgetrestriktion (2.50) gilt $(1+n) \cdot d g_{t+1}=-d \tau_{t}>0$. Ab Periode $t+1$ werden die Steuern so festgesetzt, daß die Pro-Kopf-Staatsverschuldung konstant ist, also $d g_{t+1}=d g_{t+1}=\cdots=d g$. Die Bruttolöhne der Periode $t$ bleiben von der Nettoneuverschuldung unberührt, da sie durch den in Periode $t-1$ akkumulierten Kapitalstock determiniert werden. Die Erhöhung der Staatsverschuldung erzeugt eine Überschußnachfrage am Kapitalmarkt, was die Zinsen steigen läßt. ${ }^{2}$ Die Leistungsbilanz des Inlands verschlechtert sich bzw. die des Auslands verbessert sich, das heißt $d v_{t}=d v_{t}=(1+n) \cdot d e_{t+1}<0$ (vgl. (2.54)). Die Erhöhung des verfügbaren Einkommens der jungen Inländer erhöht zwar deren Ersparnis, diese wird aber von der Erhöhung der Staatsverschuldung überkompensiert. In Periode $t+1$ reduzieren sich weltweit sowohl die Brutto- als auch die Nettolöhne. Der Grund liegt in der Erhöhung von $r_{t+1}$, welche sowohl die Kapitalrendite als auch den Zinsendienst der Staatsverschuldung in beiden Ländern erhöht (vgl. (2.40)). Im Zeitverlauf steigt der Zinssatz weiter an, und die inländische Leistungsbilanz verschlechtert sich zunehmend. Ein in einer Periode auftretendes staatliches Budgetdefizit induziert somit einen Anpassungsprozeß, der zu einem neuen langfristigen Gleichgewicht führt. In diesem hat sich das verfügbare Einkommen, gegenüber dem Niveau im alten langfristigen Gleichgewicht, weltweit reduziert, und der Zinssatz hat sich erhöht. Die inländische Leistungsbilanz hat sich verschlechtert bzw. die ausländische verbessert.

Die Auswirkung des Budgetdefizits auf die Wohlfahrt der Akteure ist wie folgt: Die anfängliche Auswirkung ist in beiden Ländern positiv. Generation $t$ profi-

\footnotetext{
${ }^{1}$ Vgl. Persson (1985), S. 79ff.

${ }^{2}$ Betrachtet man die auf dem Kapitalmarkt gehandelten Vermögenstitel, so läßt sich die positive Nettoneuverschuldung als eine Vermehrung der sich im Umlauf befindenden Vermögenstitel interpretieren. Diese Überangebot an Vermögenstiteln führt zu einer Reduktion ihres Preises. Der Preis und die Rendite von Vermögenstiteln verhalten sich invers zueinander. Somit ist die Preisreduktion gleichbedeutend mit einer Erhöhung des Zinssatzes.
} 
tiert von dem höheren Zinssatz, welcher die Rendite ihrer Ersparnis erhöht, und die Inländer dieser Generation zusätzlich durch die Erhöhung des verfügbaren Einkommens. Die Auswirkung auf die Wohlfahrt von Generation $t+1$ ist unbestimmt. Der Zinssatz steigt, und das verfügbare Einkommen reduziert sich weltweit. Die Reduktion des Nettolohns resultiert aus der weltweiten Reduktion des Bruttolohns und der höheren Steuerlast. Im Inland erhöht sich die Besteuerung als Folge der erhöhten öffentlichen Verschuldung und der Erhöhung des Zinssatzes. Ist der ausländische Staat verschuldet, so erhöht sich auch dort die Besteuerung des Arbeitseinkommens, da sich der Zinsendienst durch den erhöhten Zinssatz vergrößert.

Die Erhöhung des Zinssatzes bewirkt einen gesellschaftlichen Umverteilungsprozeß des Einkommens von den Arbeitern und Steuerzahlern zu den Vermögensbesitzern. Ist die Auslandsposition negativ, sind einige dieser Vermögensbesitzer Ausländer, was die nationalen Konsummöglichkeiten reduziert. In diesem Fall verschlechtert die Staatsverschuldung die Lage zukünftiger Generationen des betreffenden Landes. Ist die Auslandsposition positiv, vergrößert die Erhöhung des Zinssàtzes die nationalen Konsummöglichkeiten. Dieser positive intertemporale Terms of Trade Effekt wirkt dem negativen Effekt der höheren Steuer und des reduzierten Kapitalstocks entgegen. Ist das Auslandsvermögen hinreichend groß, ist es dann sogar möglich, daß das staatliche Budgetdefizit die Wohlfahrt der zukünftigen Generationen des betreffenden Landes erhöht. Der Unterschied zwischen dem Land, dessen Staat sich verschuldet, und dem anderen Land besteht darin, daß die zukünftigen Generationen des ersten Landes zusätzlich vom direkten Steuereffekt der erhöhten Staatsschuld negativ betroffen werden. Es ist also möglich, daß die Erhöhung der Staatsverschuldung in einem Land die Wohlfahrt zukünftiger Generationen in beiden Ländern langfristig reduziert. Es ist auch möglich, daß die Wohlfahrt der Akteure in einem der Länder langfristig erhöht wird. Eine langfristige Erhöhung der Wohlfahrt in beiden Ländern ist allerdings nicht möglich. Dies ist auch verständlich, da die Staatsschuld Produktivkapital als Anlageinstrument verdrängt. In einem dynamisch effizienten Gleichgewicht $(r>n)$ bewirkt dies eine Reduktion der Kapitalintensität weg von dem der goldenen Regel entsprechenden Niveau. Die langfristigen Konsummöglichkeiten der Weltwirtschaft reduzieren sich somit. 
Lin (1994a) untersucht die langfristigen Auswirkungen von staatlichen Budgetdefiziten auf die Leistungs- und die Handelsbilanz, unter dem Aspekt sich international unterscheidender Zeitpräferenzraten. Wie bei Buiter (1981) unterscheiden sich die beiden Länder nur bezüglich der Zeitpräferenzrate der sie bevölkernden Akteure. Im Gegensatz zu Buiter nimmt Lin allerdings einen sich nach einer Periode vollständig abschreibenden Kapitalstock an, also $\delta=1$. (2.28) wird somit zu $r_{t}=f^{\prime}\left(k_{t}\right)-1$ (Realzins = Nettogrenzprodukt des Kapitals) und es gilt $f\left(k_{t}\right)=w_{t}+\left(1+r_{t}\right) \cdot k_{t}$. Wie bei Persson (1985) besteuert der Staat die jungen Akteure und finanziert ein gegebenenfalls auftretendes Budgetdefizit mittels einperiodiger Staatsanleihen. Anders als bei Persson findet ein öffentlicher Verbrauch von Gütern statt. ${ }^{1}$ Sei der nationale reale Pro-Kopf-Staatskonsum der Periode $t$ mit $c_{t}^{g}$ definiert, dann lautet die staatliche Budgetrestriktion:

$$
c_{t}^{g}+\left(1+r_{t}\right) \cdot g_{t}=\tau_{t}+(1+n) \cdot g_{t+1} \text {. }
$$

(2.60) unterscheidet sich von (2.50) nur bezüglich der Konsumkomponente $c_{t}^{g}$. (2.60) läßt sich umformen:

$$
\underbrace{(1+n) \cdot g_{t+1}-g_{t}}_{\text {Budgetdefizit }}=\underbrace{r_{1} \cdot g_{t}}_{\text {Zinsendienst }}+\underbrace{c_{t}^{g}-\tau_{t}}_{\begin{array}{c}
\text { primaires } \\
\text { Budgeldefizit }
\end{array}} .
$$

Das staatliche Budgetdefizit setzt sich aus dem primären Budgetdefizit, also der Differenz zwischen Konsumausgaben und Steuereinnahmen, und dem Zinsendienst zusammen. Eine Steuererhöhung reduziert das Budgetdefizit bzw. eine Steuerreduktion erhöht das Budgetdefizit.

Die Handelsbilanz ist die Differenz zwischen Inlandsproduktion und Absorption:

${ }^{1}$ Es wird hierbei angenommen, daß der Staatskonsum keinen Einfluß auf den Grenznutzen des Privatkonsums der Akteure besitzt, daß er also für das individuelle Konsum- und Sparverhalten der Akteure irrelevant ist (die Optimalitätsbedingung (2.26) hat weiterhin Gültigkeit). Dies ist zum Beispiel dann der Fall, wenn der Staatskonsum nicht nutzenstiftend ist, aber auch dann, wenn er additiv separabler Form ist, das heißt $u\left(c_{t}^{\prime}, c_{t+1}^{\prime}\right)+v\left(c_{t}^{8}\right)+v\left(c_{t+1}^{g}\right)$. Man erkennt, daß der Staatskonsum im zweiten Fall den Charakter eines öffentlichen Gutes besitzt (Nichtausschließbarkeit vom Konsum und Nichtrivalität im Konsum). 


$$
h_{t}=f\left(k_{t}\right)-c_{t}^{\prime}-\frac{c_{t}^{t-1}}{1+n}-c_{t}^{g}-(1+n) \cdot k_{t+1} \text {. }
$$

Vergleicht man (2.62) mit (2.31) so erkennt man, daß die Absorption durch den Staatskonsum $c_{t}^{g}$ erhöht wird. Da der Kapitalstock per Annahme im Produktionsprozeß vollständig verschlissen wird $(\delta=1)$, ist die Kapitalbildung einer Periode gleich dem Kapitalstock der folgenden Periode. ${ }^{1}$ Die Leistungsbilanz ist gleich der Handelsbilanz zuzüglich dem Ertrag aus dem Auslandsvermögen (vgl. (2.55)), also:

$$
v_{t}=h_{t}+r_{t} \cdot e_{t} \text {. }
$$

Wie aus (2.61) folgt, erfüllt das staatliche Budget im langfristigen Gleichgewicht:

$$
(n-r) \cdot g=c^{g}-\tau
$$

Gilt $r>n(r<n)$, ist eine höhere Steuer, bei unverändertem Pro-Kopf-Staatskonsum, mit einer höheren (niedrigeren) Staatsverschuldung verbunden. Im Fall $r=n$ ist das Steueraufkommen gleich dem Staatskonsum. Der Zusammenhang erklärt sich wie folgt: Gilt $r>n$, reicht die Nettoneuverschuldung des Staates, welche die Pro-Kopf-Staatsverschuldung konstant läßt, nicht aus dem Zinsendienst nachzukommen. Die Finanzierungslücke wird durch die Besteuerung der jungen Akteure geschlossen. Je höher die Staatsverschuldung ist, desto höher muß die Steuer sein. Gilt $r=n$, reicht das Wachstum der Bevölkerung gerade aus, durch die Nettoneuverschuldung den Zinsendienst zu finanzieren. Die Steuereinnahmen werden vollständig konsumiert. Gilt $r<n$, überkompensiert die Nettoneuverschuldung des Staates seinen Zinsendienst, so daß ein Teil davon für den Staatskonsum verwendet werden kann. Je höher die Staatsverschuldung ist, desto größer ist dieser Teil. Das zur Finanzierung des Staatskonsums

\footnotetext{
${ }^{1}$ Sei die Pro-Kopf-Bruttoinvestition der Periode $t$ mit $i_{t}$ definiert, dann gilt $(1+n) \cdot k_{t+1}$ $=i_{t}+(1-\delta) \cdot k_{t}$ und damit $i_{t}-\delta \cdot k_{t}=(1+n) \cdot k_{t+1}-k_{t}$, also Bruttoinvestition - Abschreibung $=$ Nettoinvestition.
} 
notwendige Steueraufkommen reduziert sich. Somit geht eine höhere Staatsverschuldung mit einer niedrigeren Steuer einher.

Die das langfristige Gleichgewicht beschreibenden Gleichungen entsprechen, bis auf die Pro-Kopf-Staatsverschuldung, (2.56)-(2.59). Aus (2.64) folgt:

$$
g=\frac{c^{g}-\tau}{n-r}
$$

Es ist klar, daß ein höherer Staatskonsum ceteris paribus mit einer höheren Staatsverschuldung bzw. einer Verschlechterung der staatlichen Vermögensposition einhergeht und damit, wie weiter oben gezeigt wurde, mit einem geringeren Auslandsvermögen.

Lin (1994a) untersucht nun die langfristigen Auswirkungen einer Steuererhöhung auf die Leistungs- und die Handelsbilanz. ${ }^{1}$ Gemäß (2.64) ist eine höhere Steuer im Fall $r>n(r<n)$ mit einer höheren (niedrigeren) Staatsverschuldung verbunden. Ist die Zeitpräferenz der Akteure in beiden Ländern gleich, dann verschlechtert eine inländische Steuererhöhung im Fall $r>n$ die inländische Leistungsbilanz und verbessert sie im Fall $r<n$. Besitzen die Inländer eine höhere Zeitpräferenz als die Ausländer, dann verschlechtert eine inländische Steuererhöhung im Fall $r>n$ die inländische Leistungsbilanz und verschlechtert oder verbessert sie im Fall $r<n$. Besitzen die Inländer eine niedrigere Zeitpräferenz als die Ausländer, dann verbessert oder verschlechtert eine inländische Steuererhöhung im Fall $r>n$ die inländische Leistungsbilanz und verbessert sie im Fall $r<n$.

Bezüglich der Handelsbilanz gilt folgendes: Ist die Zeitpräferenz der Akteure in beiden Ländern gleich, dann verbessert eine inländische Steuererhöhung die

\footnotetext{
${ }^{1}$ Vgl. Lin (1994a), S. 297ff. Bei Lin ist die Lohnsteuer die exogene Politikvariable und nicht wie bei Persson (1985) die Staatsverschuldung. Bei Persson werden die Steuern so festgesetzt, daß die aus einem Transfer resultierende Erhöhung der Staatsverschuldung über die Zeit konstant ist. Bei Lin wird die Lohnsteuer erhöht und in den folgenden Perioden auf dem erhöhten Niveau belassen. Die Staatsverschuldung wird dann endogen bestimmt. Auf (2.61) ist klar, daß die Steuererhöhung das staatliche Budgetdefizit reduziert.
} 
inländische Handelsbilanz. Besitzen die Inländer eine höhere Zeitpräferenz als die Ausländer, dann verbessert oder verschlechtert eine inländische Steuererhöhung die inländische Handelsbilanz. Besitzen die Inländer eine niedrigere Zeitpräferenz als die Ausländer, dann verbessert oder verschlechtert eine inländische Steuererhöhung im Fall $r>n$ die inländische Handelsbilanz und verbessert sie im Fall $r<n$.

Die politischen Implikationen sind folgende: Bei sich international nicht unterscheidenden Zeitpräferenzraten verbessert eine Steuererhöhung in jedem Fall die Handelsbilanz und verschlechtert (verbessert) die Leistungsbilanz im Fall, daß der Realzins höher (niedriger) als die Wachstumsrate der Weltwirtschaft ist. Unterscheiden sich die Zeitpräferenzraten international, muß eine höhere Steuer im Land mit den geduldigeren Akteuren nicht zwangsläufig zu einer langfristig verschlechterten Leistungsbilanz führen. Die gleiche Politik führt im Land mit den ungeduldigeren Akteuren eher zu einer Verschlechterung der Leistungsbilanz (bei $r>n$ auf jeden Fall und bei $r<n$ vielleicht). Im Land mit den ungeduldigeren Akteuren führt eine höhere Steuer nicht zwangsläufig zu einer langfristigen Verbesserung der Handelsbilanz. Im Land mit den geduldigeren Akteuren führt die gleiche Politik eher zu einer langfristigen Verbesserung der Handelsbilanz (bei $r<n$ auf jeden Fall und bei $r<n$ vielleicht).

\subsubsection{Der Einfluß des öffentlichen Budgets}

\section{auf die Auslandsposition}

Schmid (1988) untersucht die Auswirkung der Fiskalpolitik auf die langfristige Auslandsposition einer Ökonomie. Er analysiert eine das Verhalten der Akteure beeinflussende, aber mit einem ausgeglichenen Staatsbudget einhergehende Politik, die auf die Auslandsposition wirkt. ${ }^{1}$ Die betrachtete Weltwirtschaft besteht aus zwei sich nur bezüglich der Fiskalpolitik der Regierungen unterscheidende OLG-Ökonomien. Der Staat besteuert die Akteure mittels einer Pauschalsteuer in der Jugend und im Alter. Ihre intertemporale Budgetrestriktion lautet damit:

\footnotetext{
'Vgl. Schmid (1988), S. 104ff.
} 


$$
c_{t}^{t}+\left(1+r_{t+1}\right)^{-1} \cdot c_{t+1}^{t}=w_{t}-\tau_{t}^{t}-\left(1+r_{t+1}\right)^{-1} \cdot \tau_{t+1}^{t}
$$

$\tau_{t}^{t}\left(\tau_{t+1}^{t}\right)$ ist die reale Pauschalsteuer, welche die Inländer der Generation $t$ in der Jugend (im Alter) zahlen müssen. Die intertemporale Budgetrestriktion der Ausländer lautet analog. Man erkennt, daß es für die Akteure, bei einem positiven Realzins, günstiger ist im Alter besteuert zu werden, da der Gegenwartswert der Steuerlast dann durch den Steuerstundungsvorteil geringer ist.

Die Ausgaben des Staates bestehen zum einen in seinem Konsum und zum anderen im gegebenenfalls anfallenden Zinsendienst. Ein auftretendes Defizit finanziert er durch die Emission einperiodiger Anleihen. Die in Pro-Kopf-Größen ausgedrückte staatliche Budgetrestriktion ist somit:

$$
\underbrace{(1+n) \cdot g_{t+1}-g_{t}}_{\text {Budgetdefzzit }}=\underbrace{r_{t} \cdot g_{t}}_{\text {Zinsendienst }}+\underbrace{c_{t}^{g}-\tau_{t}^{t}-(1+n)^{-1} \cdot \tau_{t}^{t-1}}_{\begin{array}{c}
\text { primüres } \\
\text { Budgetdefzit }
\end{array}} \text {. }
$$

Im langfristigen Gleichgewicht mit positivem Staatskonsum $\left(c^{g}>0\right)$ und ohne Staatsverschuldung $(g=0)$ ist das staatliche Budget in jeder Periode ausgeglichen und es gilt $c^{g}=\tau$. Je größer das staatliche Budget ist, desto geringer ist die Kapitalintensität, da der öffentliche Verbrauch Produktivkapital verdrängt. Der Staatskonsum wird durch die Besteuerung der Akteure finanziert, deren verfügbares Einkommen sich dadurch reduziert. Diese Einkommensreduktion bewirkt eine Reduktion der Ersparnis und damit der Kapitalbildung. Ist das staatliche Budget in beiden Ländern ausgeglichen und gleich groß $\left(c^{g}=C^{8}\right)$, so gilt dies auch für die Auslandsposition $(e=E=0)$, da der öffentliche Sektor der einzige Aspekt ist, in dem sich die beiden Länder per Annahme unterscheiden. Unterscheiden sich die staatlichen Budgets bezüglich ihrer Größe, dann ist das Land mit dem größeren öffentlichen Sektor ein Schuldnerland und das andere ein Gläubigerland. Je höher die Steuer ist, desto geringer ist das Nationalvermögen, da die Ersparnis der Akteure mit sinkendem verfügbaren Einkommen abnimmt.

Eine weitere mit einem ausgeglichenen Budget einhergehende Politik ist die Implementierung eines Sozialversicherungssystems nach dem Umlageverfah- 
ren. Ist dies der einzige Eingriff des Staates in die Ökonomie und ist er unverschuldet, dann erfüllt sein Budget langfristig:

$$
\tau_{t}^{t-1}=-(1+n) \cdot \tau_{t}^{t}, \quad \tau_{t}^{t-1}<0, \tau_{t}^{t}>0
$$

Die Jungen zahlen in jeder Periode einen Beitrag in Höhe von $\tau_{t}^{t}$ in die Sozialversicherung ein, und die Alten erhalten in jeder Periode eine Rente in Höhe von $-\tau_{t}^{t-1}$. Der Sozialversicherungsbeitrag ist eine die jungen Akteure belastende Pauschalsteuer und die Rente ein den alten Akteuren zukommender Pauschaltransfer. Unter Berücksichtigung des Bevölkerungswachstums gilt im Aggregat $L_{t} \cdot \tau_{t}^{t}=-L_{t-1} \cdot \tau_{t}^{t-1}$. Das Sozialversicherungssystem weist in keiner Periode ein Finanzierungsdefizit auf. Je höher der Sozialversicherungsbeitrag ist, desto geringer ist die Ersparnis der Akteure, da dieser ihr verfügbares Einkommen reduziert. Die für das Alter erwartete Rente reduziert die Ersparnis zusätzlich. Somit wirkt sich die Implementierung des Sozialversicherungssystems auf die Kapitalbildung und die Auslandsposition in gleicher Weise wie der Steuer finanzierte Staatskonsum aus. Je größer die Sozialversicherungssysteme sind, desto kleiner ist der Kapitalstock. ${ }^{1}$ Unterscheiden sich die Sozialversicherungssysteme bezüglich ihrer Größe international, dann ist das Land mit dem größeren (kleineren) Sozialversicherungssystem ein Schuldnerland (Gläubigerland). ${ }^{2}$

' Die Frage, ob der Übergang von einem Rentensystem nach dem Umlageverfahren zu einem kapitalgedeckten Rentensystem zu einer Pareto-Verbesserung führt, versucht Breyer (1998) zu beantworten. Die Wohlfahrtswirkung hängt von der konkreten Ausgestaltung des Rentensystems und der Modellstruktur ab. Sowohl eine Pareto-Verbesserung als auch eine Pareto-Verschlechterung sind möglich.

${ }^{2}$ Es sei hier auch auf Fisher (1995) hingewiesen. Fisher untersucht den Zusammenhang zwischen ökonomischem Wachstum, Handelsströmen und die Nachfrage beeinflussender Fiskalpolitik (Pauschalsteuern und Pauschaltransfers). In seinem Modell nimmt Fisher eine ZweiSektoren-Technologie an. Im einen Sektor wird das Konsumgut produziert und im anderen das Kapitalgut, und es besteht eine Heckscher-Ohlin-Außenhandelsstruktur. Der Kapitalstock unterliegt einer positiven Abschreibungsrate. Das Wachstum der Weltwirtschaft wird vom Sparverhalten in beiden Ländern determiniert. Eine Erhöhung der ökonomischen Wachstumsrate läßt sich gegebenenfalls mittels einer intragenerationalen Umverteilungspolitik, welche zu keiner Verdrängung von Kapitalinvestitionen führt, herbeiführen. 


\subsubsection{Kapitalertragsteuern und das internationale Steuersystem}

In einer integrierten Weltwirtschaft mit vollständiger Kapitalmobilität stimmt der Standort des Kapitals nicht zwangsläufig mit seinem Eigentum überein. Inländisches Kapital kann sich in ausländischem Eigentum befinden und umgekehrt. Will man die Konsequenzen einer Besteuerung des Kapitals eruieren, muß man zwischen zwei grundlegenden Steuersystemen unterscheiden: Im ersten System findet die Besteuerung nach dem Wohnsitzstaatprinzip, auch Weltoder Universalprinzip genannt, statt. Die Steuer wird in dem Land erhoben, in dem der Einkommensbezieher seinen Wohnsitz hat, unabhängig davon in welchem Land das zu versteuernde Einkommen entsteht. Im zweiten System findet die Besteuerung nach dem Quellenstaatprinzip, auch Territorialprinzip genannt, statt. Der Kapitalertrag wird in dem Land versteuert, in dem er entsteht, unabhängig davon welcher Nationalität der Einkommensbezieher ist. ${ }^{1}$

Im Folgenden werden die Auswirkungen von Kapitalertragsteuern dargestellt. Die Entlohnung der Produktionsfaktoren Kapital und Arbeit wird weiterhin durch die Beziehungen (2.28) und (2.29) gegeben. Sei $\tau_{t}^{W}\left(T_{t}^{W}\right)$ der inländische (ausländische) Steuersatz auf die Kapitalerträge nach dem Wohnsitzstaatprinzip und $\tau_{t}^{Q}\left(T_{t}^{Q}\right)$ der inländische (ausländische) Steuersatz auf die Kapitalerträge nach dem Quellenstaatprinzip. Die Arbitragefreiheit im Gleichgewicht bedingt aus Sicht der Inländer:

$$
\left(1-\tau_{t}^{W}-\tau_{t}^{Q}\right) \cdot r_{t}=\left(1-\tau_{t}^{W}-T_{t}^{Q}\right) \cdot R_{t}
$$

und aus Sicht der Ausländer:

$$
\left(1-T_{t}^{W}-\tau_{t}^{Q}\right) \cdot r_{t}=\left(1-T_{t}^{W}-T_{t}^{Q}\right) \cdot R_{t}
$$

Auf der linken Seite steht die in der Periode $t$ im Inland erzielbare Nettorendite und auf der rechten Seite die im Ausland erzielbare. Gilt (2.69) ((2.70)) sind die Inländer (Ausländer) indifferent zwischen einer Investition im Inland und einer

\footnotetext{
${ }^{1}$ Vgl. Frenkel u.a. (1991), S. 22ff.
} 
Investition im Ausland. Bei Verletzung der Bedingungen (2.69) und (2.70), ist es den Akteuren möglich, sich im Land mit der niedrigeren Nettorendite unbegrenzt zu verschulden und das aufgenommene Kapital im Land mit der höheren Nettorendite $\mathrm{zu}$ investieren, was unbegrenzte Gewinne erzielbar macht. Die Arbitragefreiheit im Gleichgewicht erfordert somit die Gültigkeit von (2.69) und (2.70). Für die Sparentscheidung der Akteure ist natürlich die Nachsteuer- also Nettorendite relevant und nicht die Bruttorendite. Somit gilt für die Ausländer $s^{t}\left[\left(1-\tau_{t}^{W}-\tau_{t}^{Q}\right) \cdot r_{t}, w_{t}\right]$ und für die Inländer $S^{t}\left[\left(1-T_{t}^{W}-T_{t}^{Q}\right) \cdot R_{t}, W_{t}\right]$.

Wenden die Regierungen das Wohnsitzstaatprinzip an, gilt also $\tau_{t}^{Q}=T_{t}^{Q}=0$, so folgt aus (2.69) und (2.70):

$$
r_{t}=R_{t}
$$

Eine Besteuerung nach dem Wohnsitzstaatprinzip bedingt somit die internationale Übereinstimmung der Bruttorenditen, welche gleich dem Realzins sind. Gemäß (2.28) und (2.29) stimmt damit auch die Kapitalintensität und das Lohnniveau in beiden Ländern überein.

Findet die Besteuerung nach dem Quellenstaatprinzip statt, gilt also $\tau_{t}^{W}=T_{t}^{W}$ $=0$, so folgt aus (2.69) und (2.70):

$$
\left(1-\tau_{t}^{Q}\right) \cdot r_{t}=\left(1-T_{t}^{Q}\right) \cdot R_{t}
$$

Eine Besteuerung nach dem Quellenstaatprinzip bedingt somit die internationale Übereinstimmung der Nettorenditen. Ist der inländische Steuersatz höher (niedriger) als der ausländische Steuersatz, so ist auch der inländische Realzins höher (niedriger) als der ausländische. Gemäß (2.28) und (2.29) ist damit die Kapitalintensität und der Lohnsatz im Inland geringer (höher) als im Ausland.

Die Arbitragebedingungen (2.69) und (2.70) lassen sich zusammenfassen:

$$
\left(1-\tau_{t}^{W}-\tau_{t}^{Q}\right) \cdot\left(1-T_{t}^{W}-T_{t}^{Q}\right)=\left(1-\tau_{t}^{W}-T_{t}^{Q}\right) \cdot\left(1-T_{t}^{W}-\tau_{t}^{Q}\right)
$$


(2.73) ist eine Art Konsistenzbedingung, welche die internationale Interdependenz der nationalen Steuersysteme manifestiert. Wenden beide Staaten das Quellenstaatprinzip bzw. das Wohnsitzsstaatprinzip an, ist Bedingung (2.73) in jedem Fall erfüllt. Wendet das Inland z.B. das Wohnsitzstaatprinzip an und das Ausland das Quellenstaatprinzip, gilt also $\tau_{t}^{W}>0, T_{t}^{Q}>0$ und $\tau_{t}^{Q}=T_{t}^{W}=0$, so folgt aus (2.73) $\tau_{t}^{W} \cdot T_{t}^{Q}=0$. Ein Gleichgewicht erfordert somit, daß der Steuersatz zumindest in einem Land null ist. Ungeachtet der Tatsache, daß die Regierungen ihr Steuersystem autonom gestalten, müssen sie das Steuersystem des anderen Landes in ihr Kalkül einbeziehen.

Sei angenommen, der Staat finanziert seinen Konsum durch die Besteuerung der Kapitalerträge und ein gegebenenfalls auftretendes Budgetdefizit durch die Emission einperiodiger Anleihen. Die in Pro-Kopf-Größen ausgedrückte Budgetrestriktion des inländischen Staates in Periode $t$ lautet dann:

$$
\begin{aligned}
& \underbrace{(1+n) \cdot g_{t+1}-g_{t}}_{\text {Budgetdefzit }}= \\
& \underbrace{r_{t} \cdot g_{t}}_{\text {Zinsendienst }}+\underbrace{c_{t}^{g}-\underbrace{\tau_{t}^{W} \cdot\left[r_{t} \cdot\left(k_{t}^{I I}+g_{t}^{I}\right)+R_{t} \cdot\left(k_{t}^{A I}+G_{t}^{I}\right)\right]}_{(2)}-\underbrace{\tau_{t}^{Q} \cdot r_{t} \cdot\left(k_{t}^{I}+g_{t}\right)}_{(1)}}_{\text {primäres Budgetdefizit }}
\end{aligned}
$$

und die des ausländischen Staates:

$$
\begin{aligned}
& \underbrace{(1+n) \cdot G_{t+1}-G_{t}}_{\text {Budgetdefizit }}= \\
& \underbrace{R_{t} \cdot G_{t}}_{\text {Zinsendienst }}+\underbrace{C_{t}^{g}-\underbrace{T_{t}^{W} \cdot\left[R_{t} \cdot\left(k_{t}^{A A}+G_{t}^{A}\right)+r_{t} \cdot\left(k_{t}^{I A}+g_{t}^{A}\right)\right]}_{(2)}-\underbrace{T_{t}^{Q} \cdot R_{t} \cdot\left(k_{t}^{A}+G_{t}\right)}_{(1)}}_{\text {primäres Budgetdefzzit }}
\end{aligned}
$$

Die staatlichen Steuereinnahmen lassen sich in zwei Quellen aufspalten. Die eine Einnahmequelle ist die mit (1) gekennzeichnete, gemäß dem Wohnsitzstaatprinzip erhobene Kapitalertragsteuer, die den gesamten Kapitalerträgen der Bürger auferlegt wird. Die andere Einnahmequelle ist die mit (2) gekennzeichnete, gemäß dem Quellenstaatprinzip erhobene Kapitalertragsteuer, die den ge- 
samten im Land erzielten Kapitalerträgen auferlegt wird. $k_{t}^{I I}\left(k_{t}^{I A}\right)$ ist hierbei das zu Beginn der Periode $t$ von den Inländern (Ausländern) gehaltene inländische Produktivkapital und $k_{t}^{A I}\left(k_{t}^{A A}\right)$ das von den Inländern (Ausländern) gehaltene ausländische Produktivkapital. Natürlich gilt $k_{t}^{I I}+k_{t}^{I A}=k_{t}^{I}$ und $k_{t}^{A I}+k_{t}^{A A}=k_{t}^{A} . k_{t}^{I}$ ist folglich die inländische Kapitalintensität zu Beginn der Periode $t$ und $k_{t}^{A}$ die ausländische. $g_{t}^{I}\left(g_{t}^{A}\right)$ ist die zu Beginn der Periode $t$ von den Inländern (Ausländern) gehaltene inländische Staatsschuld und $G_{t}^{I}$ $\left(G_{t}^{A}\right)$ die von den Inländern (Ausländern) gehaltene ausländische Staatsschuld. Es gilt $g_{t}^{I}+g_{t}{ }^{A}=g_{t}$ und $G_{t}^{I}+G_{t}^{A}=G_{t} \cdot g_{t}$ ist wie bisher die inländische ProKopf-Staatsverschuldung zu Beginn der Periode $t$ und $G_{t}$ die ausländische.

Es gibt weiterhin zwei Weltmärkte und zwar den Weltgütermarkt und den Weltkapitalmarkt. Für die Gleichgewichtsbetrachtung reicht es wieder aus sich auf einen Markt zu konzentrieren, da die zweite Gleichgewichtsbedingung redundant ist (Walras-Gesetz). Die in Pro-Kopf-Größen ausgedrückte Gleichgewichtsbedingung für den Weltkapitalmarkt der Periode $t$ lautet:

$$
\underbrace{s^{t}+S^{t}}_{\text {private Ersparnis }}-\underbrace{\left(g_{t+1}+G_{t+1}\right) \cdot(1+n)}_{\text {offentliche Verschuldung }}=\underbrace{\left(k_{t+1}^{I}+k_{t+1}^{A}\right) \cdot(1+n)}_{\text {Kapitalstock }} .
$$

Die aggregierte Ersparnis in Periode $t$ muß gleich dem Weltkapitalstock zu Beginn der Periode $t+1$ sein. Die öffentliche Verschuldung reduziert die aggregierte Ersparnis oder verdrängt anders ausgedrückt das Produktivkapital im Portfolio der Anleger. Der Unterschied zwischen (2.76) und (2.51) ist, daß sich die Kapitalintensität, bei einer Besteuerung der Kapitalerträge nach dem Quellenstaatprinzip, international unterscheiden kann.

Lin (1994b) untersucht, im Rahmen eines wie hier beschriebenen Modells, die langfristigen Auswirkungen der Kapitalertragsteuern auf die Bruttorendite (= Realzins), die Nettorendite (= Zins nach Steuern) und die Kapitalakkumulation. ${ }^{1}$ In einem System der Besteuerung nach dem Wohnsitzstaatprinzip reduziert eine Erhöhung des Steuersatzes in einem Land, im Fall einer Nettorendite, die höher als die Wachstumsrate der Bevölkerung ist, die Brutto- und die Nettoren-

${ }^{1}$ Vgl. Lin (1994b), S. 133ff. 
dite und erhöht die Kapitalintensität in beiden Ländern. Ist das Sparverhalten der Akteure des anderen Landes hinreichend zinselastisch, so gilt das eben Gesagte auch im Fall eines Systems der Besteuerung nach dem Quellenstaaprinzip. Die Intuition ist offensichtlich: Eine Erhöhung des Steuersatzes auf Kapitalerträge reduziert ceteris paribus die Neuverschuldung des Staates. Die Mittelaufnahme des Staates am Kapitalmarkt nimmt somit ab, was ein Überschußangebot an Kapital erzeugt. Der Anlagebedarf der Akteure, also ihre Ersparnis, trifft auf weniger Anlagemöglichkeiten, was deren Bruttorendite sinken läßt. Die Reduktion der Bruttorendite geht mit einer Erhöhung der Kapitalintensität und einer Reduktion der Nettorendite einher. Im Fall einer Nettorendite, die geringer als die Wachstumsrate der Bevölkerung ist, kann es allerdings auch dazu kommen, daß eine Erhöhung des Zinssatzes die Bruttorendite erhöht und die Kapitalintensität reduziert.

Die Ergebnisse aus Lin (1994b) resultieren offensichtlich aus der Tatsache, daß die Kapitalertragsteuer hier einen direkten Einfluß auf das staatliche Budget und die Staatsverschuldung besitzt. Es ist zu erwarten, daß eine Veränderung der Kapitalertragsteuer, die auf die Höhe von Transfers, anderer Steuern oder des Staatskonsums wirkt, zu anderen Ergebnissen führt, und dem ist auch so.

Im Modell von Sibert (1990) besteuert der Staat das Kapital und verwendet das Steueraufkommen zur Finanzierung von Transfers. Eine in einem Land stattfindende Erhöhung der Steuer auf die Ersparnis erhöht hier langfristig den Zinssatz und reduziert den Lohnsatz und die Kapitalintensität in beiden Ländern. Eine in einem Land stattfindende Erhöhung der Investitionssteuer kann die Kapitalakkumulation in dem betreffenden Land langfristig reduzieren und die Kapitalakkumulation im anderen Land reduzieren, aber auch erhöhen. ${ }^{1}$

Ihori (1991) untersucht die Effekte einer aufkommensneutralen Steuerreform. Der Staat besteuert den Kapitalertrag und den privaten Konsum. Das Steueraufkommen verwendet er zur Finanzierung seines eigenen Konsums. In einem Sy-

${ }^{1}$ Vgl. Sibert (1990), S. 303ff. Die Steuer auf die Ersparnis ist eine Besteuerung nach dem Wohnsitzstaatprinzip und die Investitionssteuer eine Besteuerung nach dem Quellenstaatprinzip. Es befinden sich keine Staatsanleihen im Umlauf, so daß alle Ersparnisse in die Kapitalakkumulation fließen. Die Investitionssteuer ist hier also eine Körperschaftsteuer. 
stem der Besteuerung nach dem Wohnsitzstaatprinzip, reduziert eine in einem Land stattfindende Erhöhung des Steuersatzes auf den Konsum, welche mit einer Reduktion des Steuersatzes auf den Kapitalertrag verbunden ist (aufkommensneutrale Steuerreform), die Bruttorendite und erhöht die Kapitalintensität in beiden Ländern. In einem System der Besteuerung nach dem Quellenstaatprinzip ist es möglich, daß die Reduktion des Steuersatzes auf den Kapitalertrag nur die Kapitalakkumulation in dem betreffenden Land erhöht und die im anderen Land sogar reduziert. ${ }^{1}$ Der Grund ist folgender: Die Reduktion der Kapitalertragsteuer läßt zwei Effekte erwarten. Eine in einem Land stattfindende Reduktion des Steuersatzes erhöht das Angebot des nach Anlagemöglichkeiten suchenden Kapitals auf dem Weltkapitalmarkt, da die Ersparnis für die Akteure des betreffenden Landes rentabler wird. Dieser Effekt wird Angebotseffekt genannt und tritt in beiden Steuersystemen auf. Die Allokation des Kapitals zu seiner rentabelsten Verwendung wird Arbitrageeffekt genannt. Der Arbitrageeffekt tritt nur im System der Besteuerung nach dem Quellenstaatprinzip auf (hier müssen die Nettorenditen international übereinstimmen (vgl. (2.72))). Die Reduktion des Steuersatzes in einem Land erhöht dort die Nettorendite. Die Erhöhung der Nettorendite im anderen Land wird durch die Reduktion der Kapitalintensität erreicht, welche die Bruttorendite erhöht. Überkompensiert nun der Arbitrageeffekt den Angebotseffekt, was bei einer hohen Substitutionselastizität zwischen Kapital und Arbeit wahrscheinlich ist, so kann es zu einem negativen Übertragungseffekt der Steuerreduktion kommen.

Sei hier auch noch auf Keen (1990) hingewiesen, der die langfristigen Auswirkungen sich international unterscheidender Kapitalertragsteuersätze auf die Wohlfahrt der Akteure untersucht. Im Gegensatz zu den bis jetzt angesprochenen Modellen nimmt Keen völlige Immobilität des Kapitals und mehrere Güter pro Periode an. Piekkola (1995) untersucht die optimale Ausgestaltung eines

\footnotetext{
${ }^{1}$ Vgl. Ihori (1991), S. 960ff. Es seien hier auch Batina und Ihori (1991) genannt, die ebenfalls die Auswirkungen einer aufkommensneutralen Steuerreform untersuchen. In ihrem Modell besteuert der Staat das Arbeitseinkommen und den privaten Konsum. Das staatliche Budget ist auch bei Batina und Ihori in jeder Periode ausgeglichen, und er verwendet das Steueraufkommen für seinen Konsum. Batina und Thori zeigen, daß eine Erhöhung der Konsumsteuer, bei endogenem Arbeitsangebot oder einem Vermächtnismotiv der Akteure, zu einer Reduktion der Kapitalakkumulation führen kann. Die in einem Land erhobene Konsumsteuer kann somit einen negativen Übertragungseffekt auf das andere Land besitzen.
} 
Steuer- und Transfersystems, unter dem Aspekt der Wohlfahrt der durch das System betroffenen Akteure. Steuerinstrumente sind hierbei Kapitalertragsteuern nach dem Wohnsitzstaat- und dem Quellenstaatprinzip. Es werden die Fälle des internationalen Steuerwettbewerbs und der Steuerkooperation betrachtet. Als Ausgangspunkt für die Analyse dient das Modell von Buiter (1981). ${ }^{1}$

\subsection{Zwei-Länder-OLG-Modelle mit fixem Produktionsfaktor}

Wie Abschnitt 2.2 gezeigt hat, wirken Außenhandel und Staatsverschuldung auf die Kapitalakkumulation, und der Kapitalstock ist eine entscheidende Zustandsvariable der Ökonomie. In diesem Abschnitt soll nun ein kurzer Überblick über die Modelle gegeben werden, in denen ein nicht reproduzierbarer Produktionsfaktor zum Produktionsergebnis beiträgt. Der Überblick ist aus dem Grund kurz gehalten, da die Diskussion in den folgenden Kapiteln im Rahmen von Modellen stattfindet, die dieses spezifische Charakteristikum aufweisen. Dort findet eine ausführliche Diskussion der Auswirkungen von Staatsverschuldung und Außenhandel statt.

Die entscheidende Eigenschaft der Modelle mit fixen Produktionsfaktoren ist, $\mathrm{da} ß$ die Wirkung von $A u ß e n h a n d e l$ und Staatsverschuldung über den Kanal der Veränderung der Akkumulationsrate eines Produktionsfaktors wegfällt. Dafür ergibt sich aber ein neuer Wirkungskanal und zwar die Veränderung des langfristigen Zinssatzes. Die Existenz eines langfristigen Zinssatzes ist ein Charakteristikum, das aus der Annahme eines nicht reproduzierbaren Produktionsfaktors resultiert. Die Bewertung eines Vermögenstitels, der das Eigentum an dem Produktionsfaktor verbrieft, erfolgt auf dem Kapitalmarkt mittels des langfristigen Zinssatzes.

\footnotetext{
${ }^{1}$ Auch Buiter und Kletzer (1991) untersuchen, ausgehend vom Buiter-Modell, die Wohlfahrtsimplikationen international koordinierter Fiskalpolitik. Allerdings beschränken sie sich auf die Implementierung eines Sozialversicherungssystems nach dem Umlageverfahren.
} 
Die Weltwirtschaft werde durch zwei Perioden lebende Generationen bevölkert, und jede Periode wird eine neue Generation geboren. Die Größe der in Periode $t$ geborenen Generation sei in jedem Land $L_{t}$. Jeder Akteur bietet in seiner Jugend eine Einheit Arbeitskraft unelastisch an, so daß $L_{t}$ das nationale Arbeitskräftepotential der Periode $t$ ist. Der zweite Produktionsfaktor neben der Arbeitskraft sei Boden. Der in jedem Land zur Verfügung stehende Boden sei in $Z$ gleich große Flächeneinheiten aufgeteilt, so daß $Z$ die zur Produktion zu Verfügung stehende nationale Bodenfläche ist. Das Nationalprodukt $X$, der Periode $t$ ist somit eine Funktion des Arbeitskräftepotentials und der Bodenfläche $Z$, also gilt $X_{t}=F\left(L_{t}, Z\right)$. Die Produktionsfunktion $F$ ist linear-homogen und läßt sich auch in Pro-Kopf-Größen ausdrücken:

$$
x_{t}=f\left(z_{t}\right) \text {, }
$$

wobei $f(0)=0, f^{\prime}\left(z_{t}\right)>0, f^{\prime \prime}\left(z_{t}\right)<0, f^{\prime}(0)=\infty$ und $f^{\prime}(\infty)=0 . x_{t}$ ist hierbei die Pro-Kopf-Produktion in Periode $t\left(=X_{t} / L_{t}\right)$ und $z_{t}$ die in Periode $t$ pro Arbeiter zu Verfügung stehenden Bodeneinheiten $\left(=Z / L_{t}=\right.$ die Bodenintensität der Produktion). ${ }^{1}$

Sei nun angenommen, daß die Bevölkerungsgröße über die Zeit konstant ist, also $L_{t}=L \forall t$. Damit ist auch die Pro-Kopf-Produktion über die Zeit konstant:

$$
x=f(z) .
$$

Auf dem Arbeitsmarkt herrscht Vollbeschäftigung, da das unelastische Arbeitsangebot der Akteure, bei völlig flexiblen Reallöhnen, vollständig nachgefragt wird. Bei Gewinnmaximierungsverhalten folgt, aufgrund der konstanten Skalenerträge und einer angenommenen kompetitiven Marktstruktur, daß die Produktionsfaktoren im Gleichgewicht mit ihrem Grenzprodukt entlohnt werden:

$$
\varepsilon=f^{\prime}(z)
$$

\footnotetext{
${ }^{1}$ Wie gehabt ist mit Pro-Kopf-Größe die Menge pro Arbeiter gemeint und nicht die Menge pro Einwohner. Beide differieren, da die Akteure per Annahme nur in ihrer Jugend arbeiten.
} 


$$
w=f(z)-z \cdot f^{\prime}(z)=x-\varepsilon \cdot z
$$

$\varepsilon$ ist der Pachtzins, den der Boden in jeder Periode erbringt und $w$ ist der ProKopf-Reallohn.

Die Akteure arbeiten in ihrer Jugend und beziehen ein Arbeitseinkommen von $w$. Einen Teil davon konsumieren sie und den Rest sparen sie gegebenenfalls. Das zur Verfügung stehende Sparinstrument sind Bodentitel. Ein Bodentitel verbrieft hierbei das Eigentum an einer Bodeneinheit. Ein Periode $t$ erworbener Bodentitel berechtigt zum Bezug des Pachtzinses aller folgenden Perioden. Die Bodentitel werden auf dem Kapitalmarkt gehandelt. Zur Bewertung der Bodentitel kapitalisieren die Marktteilnehmer den Pachtzinsstrom mit dem Kapitalmarktzins:

$$
p_{t}=\frac{\varepsilon}{r_{t+1}^{l}}
$$

$p_{t}$ ist somit der Realpreis eines Bodentitels in der Periode $t$ und $r_{t+1}^{l}$ der von den Marktteilnehmern in dieser Periode bei ihrer Bewertung zugrunde gelegte Kapitalmarktzins.

Sei angenommen, daß die Akteure vollkommene Voraussicht besitzen. Der von ihnen erwartete Bodentitelpreis ist somit gleich dem tatsächlichen Bodentitelpreis, also $p_{t+1}^{e}=p_{t+1}, \forall t$. Ein von einem Akteur in Periode $t$ erworbener Bodentitel erbringt in Periode $t+1$ den Pachtzins $\varepsilon$ und den Verkaufserlös $p_{t+1}$. Somit ist die Verzinsung der Ersparnis eines Akteurs der Generation $t$ :

$$
\frac{\varepsilon+p_{t+1}}{p_{t}}=1+r_{t+1}
$$

$r_{t+1}$, der kurzfristige Zinssatz, ist somit die Rendite der Ersparnis der Periode $t$. Er ist der für die Sparentscheidung relevante Zinssatz. $r_{t+1}^{l}$, der Kapitalmarktzins, ist der Zinssatz, der den Preis (= Kurswert) des Bodentitels in Periode $t$ determiniert. In jeder Periode gibt es somit zwei Zinssätze und damit eine Zinsstruktur. 
Das Modell von Fried und Howitt (1988) entspricht von der Produktionsseite dem hier beschriebenen, die Produktionsfaktoren sind also Arbeit und Boden. Der Staat greift in den Wirtschaftskreislauf durch Pauschalsteuern bzw. Pauschaltransfers ein, die den jungen Akteuren auferlegt werden bzw. ihnen zukommen. Ein gegebenenfalls auftretendes Budgetdefizit finanziert er durch die Emission ewiger Anleihen. Fried und Howitt untersuchen nun die Auswirkungen der Fiskalpolitik auf die Wohlfahrt, den Zinssatz und die Zahlungsbilanz. Sie zeigen, daß ein von den Akteuren antizipiertes zukünftiges staatliches Budgetdefizit zu einer Reduktion ihrer Wohlfahrt und des kurzfristigen Zinssatzes führt und zu einer Erhöhung des langfristigen Zinssatzes. ${ }^{1}$

Im Modell von Kareken und Wallace (1977) wird eine Zwei-Sektoren-Technologie angenommen. In jedem Sektor wird ein Konsumgut unter Einsatz von Boden und Arbeit produziert. Die Außenhandelsstruktur ist vom Heckscher-OhlinTyp. Kareken und Wallace vergleichen nun zwei Politikregime unter dem Aspekt der Wohlfahrt der Akteure. ${ }^{2}$ Im Laissez-Faire-Regime werden beide Güter frei gehandelt, und den Akteuren ist es möglich Bodentitel des anderen Landes zu erwerben. Im Portfolio-Autarkie-Regime findet zwar auch ein freier Güteraustausch statt, der Erwerb von Bodentiteln des anderen Landes ist aber nicht möglich. Das langfristige Gleichgewicht unter dem Laissez-Faire-Regime ist Pareto-optimal, das langfristige Gleichgewicht unter dem Portfolio-AutarkieRegime nicht. Unterscheiden sich die kurzfristigen Zinssätze international, was unter dem Portfolio-Autarkie-Regime möglich ist, dann gibt es mindestens eine weitere Gleichgewichtsallokation, die eine Pareto-Verbesserung darstellt.

Im Modell von Dornbusch (1985) ist Arbeit der einzige Produktionsfaktor. Die Akteure bieten ihre Arbeitskraft in ihrer Jugend unelastisch an und beziehen hierfür ein Arbeitseinkommen. Einen Teil ihres Einkommens konsumieren sie, und den Rest sparen sie. Das Produktionsergebnis ist verderblich und das einzige Sparmedium sind sich im Umlauf befindende ewige Staatsanleihen. Eine Anleihe ist mit einem Coupon von $\varepsilon$ Gütereinheiten pro Periode verbunden. Die Anleihen werden ex Coupon gehandelt, so daß ihr Kurswert gleich dem in Gleichung (2.81) bestimmten Preis ist. Die Verzinsung der Ersparnis der Akteu-

\footnotetext{
${ }^{1} \mathrm{Vgl}$. Fried und Howitt (1988), S. 15ff.

${ }^{2}$ Vgl. Kareken und Wallace (1977), S. 33ff.
} 
re wird wieder durch (2.82) gegeben. Das Modell von Dornbusch ist somit formal äquivalent zu dem hier beschriebenen Modell. Dornbusch zeigt, daß die Öffnung zum Weltmarkt, unter der Voraussetzung, daß der durch die Öffnung induzierte Umverteilungsprozeß durch einen Transfermechanismus kompensiert wird, zu einer Pareto-Verbesserung führt. ${ }^{1}$

'Vgl. Dornbusch (1985), S. 133ff. 
Michael Ganske - 978-3-631-75472-6

Downloaded from PubFactory at 01/11/2019 04:35:58AM

via free access 


\section{Ein Modell einer OLG-Ökonomie mit Produktion}

Die Geschichte der Ökonomie wird durch die diskreten Perioden $t \in Z$ indiziert. Sie beginnt in $-\infty$ und reicht bis $+\infty .{ }^{1}$ Jede Periode wird eine neue Generation geboren, die zwei Perioden lebt. ${ }^{2}$ Die jeweilige Generation wird dabei durch ihre erste Lebensperiode indiziert. Jede Generation besteht aus einem einzelnen repräsentativen Akteur, der durch einen Einkommensvektor und eine Präferenzordnung charakterisiert wird. Aufgrund der demographischen Struktur leben in jeder Periode zwei Akteure. Das ist zum einen der Alte, der sich in seiner zweiten Lebensperiode befindet, und zum anderen der Junge, der sich in seiner ersten Lebensperiode befindet. Die Struktur der Ökonomie sei stationär, das heißt, jede neue Generation ist eine Replikation der alten. Die Akteure aller Generationen sind somit, in bezug auf ihren Einkommensvektor und ihre Präferenzordnung, identisch.

Die Präferenzordnung der Akteure kann vermöge einer intertemporalen Nutzenfunktion repräsentiert werden. Argumente sind hierbei der Konsum in der Jugend und im Alter. $u\left(c_{t}^{t}, c_{t+1}^{t}\right)$ beschreibt somit den Nutzen des Akteurs $t$, resultierend aus dem Konsum in der Jugend $c_{t}^{t}$ (also in Periode $t$ ) und dem Konsum im Alter $c_{t+1}^{t}$ (also in Periode $t+1$ ). Die Nutzenfunktion ist streng qua-

${ }^{1}$ Die Annahme einer doppelt infiniten zeitlichen Struktur vereinfacht die Diskussion, da so keine erste Generation existiert, die gesondert modelliert werden muß; siehe dazu auch den Survey von Geanakoplos (1987a), in dem sowohl OLG-Modelle mit doppelt infiniter Struktur als auch OLG-Modelle mit endlicher Anfangsperiode dargestellt werden. Wie Geanakoplos und Polemarchakis (1991) zeigen, läßt sich eine OLG-Ökonomie, mit einer doppelt infiniten zeitlichen Struktur, in eine äquivalente OLG-Ökonomie, mit endlicher Anfangsperiode, transformieren; vgl. Geanakoplos und Polemarchakis (1991), S. 1913f.

${ }^{2}$ Die Annahme von zwei Lebensperioden erscheint restriktiv, dies ist jedoch nicht der Fall. Balasko et al. (1980) entwickeln eine einfache Methode, die es ermöglicht, Generationen und Perioden eines Modells, in dem die Akteure eine beliebige aber endliche Anzahl von Perioden leben, derart umzudefinieren, daß eine demographische Struktur entsteht, in der die Akteure zwei Perioden leben; vgl. Balasko et al. (1980), S. $319 \mathrm{ff}$. 
sikonkav, differenzierbar und streng ansteigend. ${ }^{1} \mathrm{Da}$ die oberen Konturmengen einer streng quasikonkaven Funktion streng konvex sind, sind die Indifferenzkurven streng konvex. Es liegt somit eine abnehmende Grenzrate der Substitution im Konsum vor. Durchschnitte werden gegenüber Extremen streng präferiert. Daß die Nutzenfunktion streng ansteigend in ihren Argumenten ist, entspricht der Vorstellung, daß die Güter nutzenstiftend sind.

Die nutzenstiftenden Güter jeder Periode sind Normalgüter. ${ }^{2}$ Die Akteure erhalten zwar keine Güterausstattung, sie verfügen in ihrer Jugend aber über eine Einheit Arbeitskraft. Die Arbeitskraft kann vermöge eines Produktionsprozesses unmittelbar in das nutzenstiftende Gut transformiert werden, dabei gilt $f(1)$ $=1 .^{3}$ Die durch die Produktionsfunktion $f(\cdot)$ beschriebene Technologie weist dabei abnehmende Skalenerträge auf. Es wird eine kompetitive Marktstruktur unterstellt, so daß die Arbeitskraft gemäß ihrem Grenzprodukt entlohnt wird. Sei $\varepsilon$ der Gewinn, dann gilt $\varepsilon=1-f^{\prime}(1)$, und damit beträgt das Arbeitseinkommen $1-\varepsilon{ }^{4}$ Der Einkommensvektor der Generationen ist somit $(1-\varepsilon, 0)$. Der in jeder Periode anfallende Gewinn von $\varepsilon$ Gütereinheiten stellt einen Dividendenstrom dar, der dem Eigentümer der Produktionstechnik zufließt.

1 Die strenge Quasikonkavität ist äquivalent zur strengen Konvexität der Präferenzen. Die Eigenschaft, daß die Nutzenfunktion streng ansteigend ist, ist äquivalent zur strengen Monotonie der Präferenzordnung. Die Differenzierbarkeit bedeutet, daß die Indifferenzkurven glatt sind. Über die Beziehung zwischen Präferenzordnung und Nutzenfunktion siehe z.B. Barten und Böhm (1982), S. 390ff.

${ }^{2}$ Normalgüter werden bei steigendem Einkommen stärker nachgefragt. Das umgekehrte gilt für inferiore Güter. Bei ihnen sinkt die Nachfrage mit steigendem Einkommen.

${ }^{3}$ Die Idee zu dieser Technologie findet sich in Mas-Collel et al. (1995), S. 770.

${ }^{4}$ Sei $l$ der Faktor Arbeit. Mit der Euler-Gleichung

$$
k \cdot y=\sum_{i=1}^{n} \partial f / \partial x_{i} \cdot x_{i}
$$

und $k<1$ (abnehmende Skalenerträge) folgt:

$$
k \cdot y=f^{\prime}(l) \cdot l \Leftrightarrow k \cdot f(l)=f^{\prime}(l) \cdot l .
$$

Mit $\varepsilon:=f(l)-k \cdot f(l)$ ergibt sich dann

$$
f(l)-\varepsilon=f^{\prime}(l) \cdot l \Leftrightarrow f(l)=f^{\prime}(l) \cdot l+\varepsilon .
$$




\subsection{Die Gleichgewichtsdynamik}

Die Akteure bieten in ihrer Jugend ihre Arbeitskraft unelastisch an. Einen Teil ihres Arbeitseinkommens $1-\varepsilon$ konsumieren sie, den anderen Teil sparen sie, um ihren Konsum im Alter zu finanzieren. Der einzige Vermögenstitel in der Ökonomie ist ein Anteilschein an der Produktionstechnik, der zum Bezug des aus dem Produktionsprozeß fließenden Dividendenstroms berechtigt. ${ }^{1}$ Akteur $t$ bietet in Periode $t$ dem Akteur $t-1$ seine Ersparnis an, im Gegenzug erhält er von diesem den Anteilschein. Er hält den Schein bis Periode $t+1$, bezieht die Dividende dieser Periode und verkauft den Schein an Akteur $t+1$, wobei er dessen Ersparnis erhält. Es findet kein Sparen in Form von Güterhortung statt, da der gleichgewichtige einperiodige Zinssatz, wie weiter unten gezeigt wird, in jeder Periode positiv ist. Die Rendite des Anteilscheins dominiert somit die Rendite der Güterhortung, welche null ist.

Sei $p_{t}$ der Realpreis des Anteilscheins in der Periode $t$ und $p_{t+1}^{e}$ der für Periode $t+1$ erwartete. Da jede Periode vor dem Verkauf des Anteilscheins die Dividende ausgeschüttet wird, ist $p$, hierbei der Preis ex Dividende. Es wird unterstellt, daß die Akteure vollkommene Voraussicht besitzen, das heißt, der erwartete Preis stimmt mit dem tatsächlichen Preis überein, also $p_{t+1}^{e}=p_{t+1}, \forall t$. Der Zusammenhang zwischen dem gegenwärtigen und dem zukünftigen Preis des Anteilscheins sieht wie folgt aus:

$$
p_{t}=\delta_{t+1} \cdot\left(\varepsilon+p_{t+1}\right), \text { wobei } \delta_{t+1}:=\frac{1}{1+r_{t+1}}
$$

${ }^{1}$ Das Modell ist von der angenommenen Technologie her formal äquivalent zu OLG-Modellen bei denen die Produktion durch den Einsatz von Arbeit und Boden erfolgt (vgl. z.B. Calvo (1978), Fried (1980) oder Fried und Howitt (1988)) oder in denen der Vermögenstitel, in dem gespart wird, eine ewige Staatsanleihe ist (vgl. Dornbusch (1985)). Entscheidend ist, daß einer der Produktionsfaktoren nicht reproduzierbar ist (der Boden oder im hier diskutierten Modell die exogen vorgegebene Produktionstechnik). Der Eigentumsanspruch an einem solchen im konstanten Umfang zur Verfügung stehenden Produktionsfaktor ist ein für immer bestehender Vermögenstitel, dessen Fundamentalwert gleich dem abdiskontierten Strom seiner Faktorerträge ist. Eine ewige Anleihe besitzt dieselben Eigenschaften. Sie ist mit einem unendlichen Strom von Couponzahlungen verbunden, die unabhängig vom Zinssatz sind. Ihr Fundamentalwert ist gleich dem abdiskontierten Strom der Couponzahlungen. 
Der Preis des Scheins in Periode $t$ ist gleich dem Gegenwartswert der Summe aus der Dividende der Periode $t+1$ und dem Verkaufserlös. $\delta_{t+1}$ ist hierbei der einperiodige Diskontfaktor, der durch den kurzfristigen Zinssatz $r_{t+1}$ determiniert wird. $1+r_{t+1}$ ist die einperiodige Verzinsung eines in Periode $t$ erworbenen Vermögenstitels. Anders ausgedrückt ist der Diskontfaktor $\delta_{t+1}$ gleich dem Verhältnis des Wertes des Anteilscheins in Periode $t$ zu seinem Wert in Periode $t+1$. Der Wert des Anteilscheins in Periode $t+1$ setzt sich aus der Dividende und dem Verkaufserlös zusammen. Da der Schein ex Dividende gehandelt wird, ist sein Wert in Periode $t$ gleich seinem Kaufpreis. Der Diskontfaktor bzw. der kurzfristige Zinssatz determinieren die Verzinsung der Ersparnis der Akteure und sind damit für ihre Sparentscheidung relevant. Der Preis des Anteilscheins aber wird durch den Kapitalmarktzins determiniert.

Der langfristige Zinssatz (= der Kapitalmarktzins) ist $r_{t+1}^{l}=\varepsilon / p_{t}$, da der Preis (= der Kurswert) des Scheins in Periode $t$ gleich dem mit dem langfristigen Zinssatz kapitalisierten Wert des unendlichen Dividendenstroms übereinstimmen muß (dies ist sein Fundamentalwert). ${ }^{1}$ Unter Verwendung von Gleichung (3.1) und $p_{t}=\varepsilon / r_{t+1}^{l}$ erhält man:

$$
r_{t+1}=\frac{\varepsilon}{p_{t}}+\frac{p_{t+1}-p_{t}}{p_{t}}=r_{t+1}^{l}-\frac{r_{t+2}^{l}-r_{t+1}^{l}}{r_{t+2}^{l}}
$$

Das erste Gleichheitszeichen spaltet die kurzfristige Rendite des Anteilscheins in den Ertrag $\left(=\varepsilon / p_{t}\right)$, auch Effektiv- oder Dividendenrendite genannt, und den Kapitalgewinn bzw. Kapitalverlust $\left(=\left(p_{t+1}-p_{t}\right) / p_{t}\right)$ auf. Das zweite Gleichheitszeichen ist eine Zinsarbitragebedingung. ${ }^{2}$ Ist der Preis des Anteilscheins über die Zeit konstant, so entsprechen sich kurzfristiger und langfristiger Zins-

' Damit wird implizit die Existenz einer spekulativen Preisblase auf den Anteilschein ausgeschlossen. Wie aus Tirole (1985), S. 1504ff, folgt, ist hierfür hinreichend, daß der kurzfristige Zinssatz positiv ist. Wie weiter unten bei der Diskussion der Modelldynamik gezeigt wird, ist diese Bedingung in jeder Periode erfüllt. Eine spekulative Preisblase auf einen Vermögenstitel ist gleich der Differenz zwischen seinem Kurswert (= Marktpreis) und seinem Fundamentalwert. Siehe auch Kehoe et al. (1991), S. 21, zum Nachweis der Nichtexistenz einer spekulativen Preisblase. Für die Herleitung der Kapitalisierungsformel (Fundamentalwert = Dividendenzahlung / langfristiger Zinssatz) siehe z.B. Brealey und Myers (1991), S. 33 und S. $47 \mathrm{ff}$. ${ }^{2}$ Zur Zinsstruktur siehe z.B. Turnovsky (1995), S. $261 \mathrm{ff}$. 
satz (man spricht von einer flachen Zinsstruktur). Verändert sich der Preis des Anteilscheins im Zeitablauf, so stimmen kurzfristiger und langfristiger Zinssatz nicht mehr überein. Sinkt der Preis des Anteilscheins, so ist der langfristige Zinssatz höher als der kurzfristige Zinssatz (man spricht von einer normalen Zinsstruktur). Steigt der Preis des Anteilscheins, so ist der kurzfristige Zinssatz höher als der langfristige Zinssatz (man spricht von einer inversen Zinsstruktur). Eine Erhöhung (Senkung) des langfristigen Zinssatzes ist ein Maß für den Kapitalverlust (Kapitalgewinn), der vollständig von dem Akteur zu tragen ist (von dem der Akteur vollständig profitiert), der zum Zeitpunkt der Zinsveränderung den Vermögenstitel hält.

Das intertemporale Nutzenmaximierungsproblem der Akteure lautet wie folgt:

$$
u\left(c_{t}^{t}, c_{t+1}^{t}\right) \rightarrow \underset{\substack{t \\ c_{t}^{\prime}, c_{t+1}^{t}}}{\operatorname{Max} !}
$$

unter der $N B$

$$
\begin{aligned}
c_{t}^{t}+s^{t} & =1-\varepsilon & & \text { (Budgetrestriktion Jugend) } \\
c_{t+1}^{t} & =\delta_{t+1}^{-1} \cdot s^{t} & & \text { (Budgetrestriktion Alter) }
\end{aligned}
$$

Aufgrund der Annahmen an die intertemporale Nutzenfunktion werden die Budgetrestriktionen mit Gleichheitszeichen erfüllt. Das Arbeitseinkommen, das die Akteure in ihrer Jugend erzielen, können sie konsumieren oder sparen. Die reale Ersparnis des Akteurs $t$ ist hierbei als $s^{t}$ definiert. Der Alterskonsum ist gleich dem Wert der Ersparnis. Die Budgetrestriktion der Jugend und die des Alters lassen sich in eine intertemporale Budgetrestriktion transformieren:

$$
c_{t}^{t}+\delta_{t+1} \cdot c_{t+1}^{t}=1-\varepsilon
$$

Der Gegenwartswert des Lebenskonsums muß gleich dem Arbeitseinkommen sein. Man erkennt, daß der Diskontfaktor $\delta_{t+1}$ auch als Gegenwartspreis des Konsums der folgenden Periode interpretiert werden kann. (3.4) ist die in Gegenwartspreisen ausgedrückte intertemporale Budgetrestriktion. Multipliziert man (3.4) mit $\delta_{t+1}^{-1}$ durch, so erhält man die in Zukunftspreisen ausgedrückte intertemporale Budgetrestriktion. $\delta_{t+1}^{-1}$ läßt sich somit als Zukunftspreis des Gegenwartskonsums interpretieren. Dies ist auch plausibel, da $\delta_{t+1}^{-1}$ die Verzinsung 
der Ersparnis ist. Je höher diese ist, desto teurer wird der Konsum in der Gegenwart im Verhältnis zum Konsum in der Zukunft.

Die zu maximierende Lagrangefunktion der Akteure lautet somit: ${ }^{1}$

$$
L=u\left(c_{t}^{t}, c_{t+1}^{t}\right)+\lambda \cdot\left(1-\varepsilon-c_{t}^{t}-\delta_{t+1} \cdot c_{t+1}^{t}\right)
$$

Die Bedingungen erster Ordnung lauten:

$$
\begin{aligned}
& \frac{\partial L}{\partial c_{t}^{t}}=\frac{\partial u\left(c_{t}^{t}, c_{t+1}^{t}\right)}{\partial c_{t}^{t}}-\lambda \stackrel{!}{=} 0, \\
& \frac{\partial L}{\partial c_{t+1}^{t}}=\frac{\partial u\left(c_{t}^{t}, c_{t+1}^{t}\right)}{\partial c_{t+1}^{t}}-\lambda \cdot \delta_{t+1} \stackrel{!}{=} 0, \\
& \frac{\partial L}{\partial \lambda}=1-\varepsilon-c_{t}^{t}-\delta_{t+1} \cdot c_{t+1}^{\prime} \stackrel{!}{=} 0 .
\end{aligned}
$$

Aufgrund der Annahmen an die Nutzenfunktion und die Budgetmenge sind die Bedingungen erster Ordnung nicht nur notwendig für ein Optimum, sondern auch hinreichend. ${ }^{2}$ Aus den Bedingungen erster Ordnung folgt:

$$
\frac{\frac{\partial u\left(c_{t}^{t}, c_{t+1}^{t}\right)}{\partial c_{t+1}^{t}}}{\frac{\partial u\left(c_{t}^{t}, c_{t+1}^{t}\right)}{\partial c_{t}^{t}}}=\delta_{t+1} \stackrel{\text { Budgetres. }}{\longrightarrow} \text { optimaler Konsumvektor }\left(c_{t}^{t}, c_{t+1}^{t}\right) \text {. }
$$

Das optimale Konsumprogramm zeichnet sich dadurch aus, daß die Grenzrate der Substitution im Konsum mit der Verzinsung der Ersparnis (= die ökonomische Substitutionsrate) übereinstimmt. Aus der Optimierung folgen die Nach-

${ }^{\prime}$ Zur Lagrange-Methode siehe z.B. Dixit (1990), S. 10ff.

${ }^{2}$ Die Budgetmenge ist konvex und die Nutzenfunktion ist streng quasikonkav; siehe dazu z.B. Takayama (1994), S. $85 \mathrm{ff}$. 
fragefunktionen der jungen und der alten Akteure, welche eine Funktion des Diskontfaktors und des Einkommens sind, also $c_{t}^{t}=c_{t}^{t}\left(\delta_{t+1}, 1-\varepsilon\right)$ und $c_{t+1}^{t}$ $=c_{t+1}^{t}\left(\delta_{t+1}, 1-\varepsilon\right)$. Da die Ersparnis gleich der Differenz aus Arbeitseinkommen und Jugendkonsum ist, ist auch sie eine Funktion des Diskontfaktors und des Einkommens. Es gilt also $s^{t}=s^{t}\left(\delta_{t+1}, 1-\varepsilon\right)$.

Ein Walras-Gleichgewicht der Ökonomie läßt sich wie folgt beschreiben:

Eine Folge von Anteilscheinpreisen $\left\{p_{t}\right\}_{t \in Z}$ und die mit ihr einhergehende Folge von Konsumplänen $\left\{\left(c_{t}^{t}, c_{t+1}^{t}\right)\right\}_{t \in Z}$ beschreiben ein Walras-Gleichgewicht der Ökonomie bei vollkommener Voraussicht, wenn die Konsumpläne die Optimalitätsbedingung (3.9) erfüllen und der Kapitalmarkt sowie der Gütermarkt in jeder Periode geräumt werden. Das heißt:

$$
\begin{aligned}
& s^{t}\left(\delta_{t+1}, 1-\varepsilon\right)=p_{t}, \forall t \\
& c_{t}^{\prime}\left(\delta_{t+1}, 1-\varepsilon\right)+c_{t}^{t-1}\left(\delta_{t}, 1-\varepsilon\right)=1, \forall t .
\end{aligned}
$$

Gleichung (3.10) beschreibt das Gleichgewicht auf dem Kapitalmarkt. In jeder Periode muß die Ersparnis des jungen Akteurs gleich dem Kurswert des vom alten Akteur angebotenen Anteilscheins sein. Gleichung (3.11) beschreibt das Gütermarktgleichgewicht. In jeder Periode muß der aggregierte Konsum des Jungen und des Alten mit der Güterproduktion der Ökonomie übereinstimmen. Aufgrund des Walras-Gesetzes reicht es aus sich auf einen Markt zu konzentrieren. Wenn ein Markt geräumt ist, ist es auch der andere. ${ }^{1}$

Die Dynamik der Ökonomie läßt sich graphisch darstellen. Darstellung 3.1 veranschaulicht die Gleichgewichtsdynamik auf dem Gütermarkt. Zu diesem Zweck wird das geometrische Instrument der Tauschkurve verwendet. ${ }^{2}$

\footnotetext{
${ }^{1}$ Zum Walras-Gesetz siehe z.B. Varian (1992), S. $317 \mathrm{f}$.

${ }^{2}$ Gale (1973) war der Erste, der das OLG-Modell unter Zuhilfenahme des Tauschkurvendiagramms diskutierte. Eine exzellente Darstellung bieten auch Cass et al. (1979). Im Anhang B wird die Herleitung einer Tauschkurve explizit durchgeführt.
} 


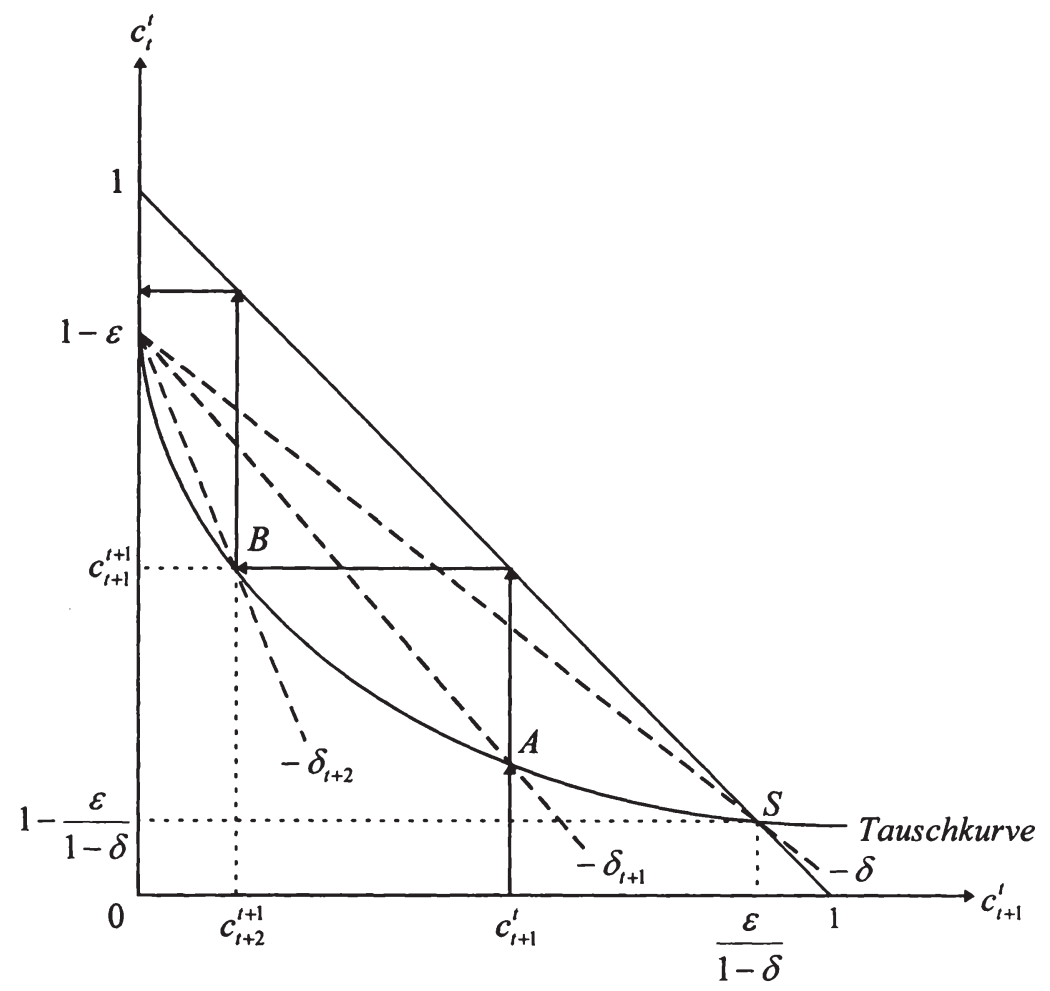

Darst. 3.1: Dynamik

Die Tauschkurve ist der geometrische Ort aller nutzenmaximierenden Konsumpläne eines Akteurs, bei gegebenem Einkommen und variierender Verzinsung der Ersparnis. ${ }^{1}$ Da jede Generation eine Replikation der vorherigen ist, genügt es eine einzige Tauschkurve herzuleiten. Mit Hilfe dieser läßt sich dann die Dynamik der Ökonomie analysieren. Auf der Ordinate ist der Konsum in der Jugend abgetragen und auf der Abszisse der Konsum im Alter. Der Einkommens-

${ }^{1}$ Die Tauschkurve wird ermittelt, indem man für alternative einperiodige Diskontfaktoren die Budgetgeraden zeichnet und die zu den jeweiligen Budgetmengen optimalen Konsumvektoren notiert. Die Verbindung der Konsumvektoren ist die Tauschkurve. Die Steigung der Budgetgerade entspricht hierbei dem Negativen des einperiodigen Diskontfaktors. 
punkt der Akteure liegt in $1-\varepsilon$ auf der Ordinate. Dies entspricht dem Arbeitseinkommen, das sie in ihrer Jugend beziehen. Ein temporäres Gütermarktgleichgewicht zeichnet sich dadurch aus, daß die Summe aus dem Konsum des jungen Akteurs und dem Konsum des alten Akteurs eins ergibt (vgl. (3.11)). Somit stellen alle Allokationen, die auf der eingezeichneten Diagonalen liegen, Gütermarktgleichgewichte dar.

Sei $\delta_{t+1}$ der Diskontfaktor zwischen den Perioden $t$ und $t+1$, dann wird der optimale Konsumvektor des Akteurs $t$ durch Punkt $A$ gegeben. Die Gütermarkträumung in Periode $t+1$ erfordert, daß der Konsumvektor des Akteurs $t+1$ in Punkt $B$ liegt. Hierfür muß der Diskontfaktor zwischen den Perioden $t+1$ und $t+2$ derart sein, daß $B$ für Akteur $t+1$ optimal ist. Der in der Darstellung eingezeichnete Diskontfaktor $\delta_{t+2}$ erfüllt diese Bedingung. Man erkennt, daß der Diskontfaktor im Zeitablauf steigt bzw. die Verzinsung fällt. Der Anteilschein verliert an Wert. ${ }^{1}$ Die Halter des Scheins müssen somit einen Kapitalverlust hinnehmen.

Der Alterskonsum des alten Akteurs $t+1 \quad c_{t+2}^{t+1}$ ist geringer als die Dividende der Periode $t+2$, so daß der Anteilschein in Periode $t+2$ einen negativen Preis $p_{t+2}$ besitzt. ${ }^{2}$ Die Tatsache, daß nach abzählbar vielen Perioden die Ökonomie in einen Bereich gerät, in dem das Anrecht auf zuküntigen Konsum einen negativen Preis besitzt, schließt eine solche Entwicklung als Gleichgewichtspfad aus. ${ }^{3}$ Es zeigt sich, daß es genau einen Gleichgewichtspfad gibt, der durchführ-

'Akteur $t$ verkauft in Periode $t+1$ für $p_{t+1}$ den Schein an Akteur $t+1$. Damit finanziert er seinen Konsum von $c_{t+1}^{t}=\varepsilon+p_{t+1} \cdot p_{t+1}$ ist gleich der Ersparnis des Akteurs $t+1$, also $1-\varepsilon-c_{t+1}^{t+1}=p_{t+1}$. Um seinen Konsum $c_{t+2}^{t+1}$ in Periode $t+2$ finanzieren zu können, muß Akteur $t+1$ den Schein in $t+2$ für $p_{t+2}$ verkaufen, denn $c_{t+2}^{t+1}=\varepsilon+p_{t+2}$. Man erkennt, daB $p_{t+1}>p_{t+2}$.

${ }^{2}$ Die Gütermarkträumung in Periode $t+2$ würde erfordern, daß sich Akteur $t+2$ in dieser Periode bei Akteur $t+1$ mit $\varepsilon-c_{t+2}^{t+1}$ Gütereinheiten verschuldet. Diese Schuld könnte jedoch nie getilgt werden, da Akteur $t+1$ in Periode $t+3$ nicht mehr lebt. Eine alternative Interpretation wäre die, daß Akteur $t+1$ dem Akteur $t+2$ die Gütereinheiten schenkt. Dies würde aber der Nutzenmaximierungshypothese widersprechen, da die Güter nutzenstiftend sind.

${ }^{3}$ Voraussetzung für diese Schlußfolgerung ist, daß sich ein Akteur dadurch besser stellen kann, daß er den Vermögenstitel einfach wegwirft. Ist diese Möglichkeit nicht gegeben, sind 
bar ist. Er ist mit dem stationären Konsumvektor $(1-\varepsilon /(1-\delta), \varepsilon /(1-\delta))$ verbunden und mit $S$ bezeichnet.

Ein langfristiges Gleichgewicht der Ökonomie zeichnet sich dadurch aus, daß der Preis des Anteilscheins sowie der Diskontfaktor über die Zeit konstant sind, also $p_{t}=p, \forall t$ und $\delta_{t+1}=\delta, \forall t$ gilt. Unter Verwendung von Beziehung (3.1) erhält man $\delta=p /(p+\varepsilon) \Leftrightarrow p=\delta \cdot \varepsilon /(1-\delta)$ Der Konsum der Jungen im langfristigen Gleichgewicht beträgt $1-\varepsilon-p=1-\varepsilon /(1-\delta)$ und der Konsum der Alten $\varepsilon+p=\varepsilon /(1-\delta)$. Die einzig mögliche Gleichgewichtsdynamik ist die, daß sich die Ökonomie unmittelbar im langfristigen mit $S$ bezeichneten Gleichgewicht $(1-\varepsilon /(1-\delta), \varepsilon /(1-\delta))$ befindet. $^{1}$

\subsubsection{Existenz eines langfristigen Gleichgewichts}

Es stellt sich nun die Frage, ob ein langfristiges Gleichgewicht immer existiert. Ein langfristiges Gleichgewicht wird durch den Schnittpunkt der Tauschkurve mit der die Gütermarktgleichgewichte beschreibenden Diagonalen gegeben. In Anhang B wird gezeigt, daß die Tauschkurve die Diagonale genau einmal schneiden muß. Hierfür verwendet man die Slutsky-Gleichung. ${ }^{2}$ Gemäß der Slutsky-Dekomposition läßt sich die Auswirkung einer Veränderung der Verzinsung der Ersparnis auf den Konsum in den beiden Lebensabschnitten in zwei Effekte aufspalten. Eine Erhöhung von $\delta_{t+1}^{-1}$ verteuert $c_{t}^{t}$ im Verhältnis zu $c_{t+1}^{t}$. Die Verteuerung bewirkt eine Verlagerung vom Konsum in der Jugend hin zum Konsum im Alter. Dies ist der intertemporale Substitutionseffekt (ISE). Der Anstieg von $\delta_{t+1}^{-1}$ bewirkt aber auch einen Einkommenseffekt (EE), da sich

Gleichgewichtspfade mit negativen Preisen möglich; siehe z.B. Brock (1990), S. 27, oder auch Kehoe et al. (1991), S. $21 \mathrm{ff}$.

${ }^{\prime}$ Auch wenn $\delta_{t+1}<\delta$ gilt, also $p_{t+1}>p$, verläßt der Gleichgewichtspfad nach abzählbar vielen Perioden den durchführbaren Bereich: Der Wert des Anteilscheins nimmt ständig zu, so daß er nach abzählbar vielen Perioden das Arbeitseinkommen der Jungen übertrifft, die ihn dann nicht mehr erwerben können.

${ }^{2}$ Vgl. dazu z.B. Varian (1992), S. 119ff. 
die Rendite der Ersparnis erhöht. ${ }^{1}$ Wegen der Normalgut-Annahme wirkt der EE in Richtung einer Erhöhung des Konsums in der Jugend und im Alter. Der ISE und der EE wirken somit gleichgerichtet auf $c_{t+1}^{t}$ in Richtung einer Erhöhung. Ihre Wirkungen auf $c_{\text {t }}^{t}$ sind gegenläufig. Es lassen sich somit zwei Fälle unterscheiden: Der erste Fall ist der, der auch in Darstellung 3.1 vorliegt. Der ISE überkompensiert den $\mathrm{EE}$ in der Jugend, so daß die Tauschkurve im gesamten Bereich eine negative Steigung aufweist. Hier spricht man davon, daß das Gut in der Jugend und das Gut im Alter Bruttosubstitute sind. Der zweite Fall ist der, daß der EE den ISE in einem Teilbereich in der Jugend überkompensiert, so daß die Tauschkurve in diesem Bereich eine positive Steigung aufweist. Hier spricht man davon, daß das Gut in der Jugend und das Gut im Alter Bruttokomplemente sind. ${ }^{2}$ In beiden Fällen weist die Tauschkurve einen elastischen Verlauf auf und schneidet die Diagonale genau einmal. Somit existiert in jedem Fall genau ein langfristiges Gleichgewicht der Ökonomie.

\subsubsection{Optimalität des langfristigen Gleichgewichts}

Es ist bekannt, daß das erste Theorem der Wohlfahrtstheorie im OLG-Modell keine Gültigkeit besitzt. ${ }^{3}$ Diese Tatsache macht eine Untersuchung der Optima-

${ }^{1}$ Je höher $\delta_{t+1}^{-1}$ ist, desto mehr Güter erhält man im Alter, bei gegebenem Konsumverzicht in der Jugend. Die durch den Einkommenspunkt gehende Budgetgerade dreht sich bei steigendem $\delta_{t+1}^{-1}$ gegen den Uhrzeigersinn und die Budgetmenge vergrößert sich. Graphisch manifestiert sich der ISE durch eine Bewegung des optimalen Konsumplans entlang einer Indifferenzkurve. Der EE spiegelt sich in einer Bewegung des optimalen Konsumplans entlang des Einkommensexpansionspfades wider. Der Einkommensexpansionspfad ist der geometrische Ort aller nutzenmaximierenden Konsumvektoren, bei gegebener Verzinsung und variierendem Einkommen; vgl. z.B. Varian (1992), S. $116 f$.

${ }^{2} \mathrm{Zu}$ der Definition von Bruttokomplementen und Bruttosubstituten siehe z.B. Nicholson (1992), S. $170 \mathrm{ff}$.

${ }^{3}$ Das erste Theorem der Wohlfahrtstheorie besagt, daß alle Walras-Gleichgewichte Paretooptimal sind; vgl. z.B. Mas-Colell et al. (1995), S. 549f. Anders als in einer Arrow-DebreuÖkonomie, können in einer OLG-Ökonomie Walras-Gleichgewichte resultieren, die Paretosuboptimal sind; siehe dazu Cass und Yaari (1966), S. 355ff. Im Gegensatz zu einer ArrowDebreu-Ökonomie (hier leben endlich viele Akteure, und der Zeithorizont ist endlich), weist eine OLG-Ökonomie eine doppelte Unendlichkeit von Akteuren und Gütern auf (aufgrund der demographischen Struktur besitzt eine OLG Ökonomie einen unendlichen Zeithorizont und 
litätseigenschaft des langfristigen Gleichgewichts notwendig. Eine Güterallokation ist durchführbar, wenn sie $c_{t}^{t}+c_{t}^{t-1} \leq 1, \forall t$ erfüllt. Eine durchführbare Güterallokation wird intertemporal optimal genannt, wenn es keine andere durchführbare Güterallokation gibt, die mindestens eine Generation besser stellt, ohne die anderen Generationen schlechter zu stellen. ${ }^{1}$ Aus Balasko und Shell (1980) folgt, daß eine Güterallokation dann intertemporal optimal ist, wenn die Zinssätze langfristig nicht negativ sind. Wie Balasko und Shell zeigen ist eine Allokation genau dann intertemporal optimal, wenn die Summe über die Güterpreise aller Perioden endlich ist. ${ }^{2}$ In der vorliegenden Arbeit wird zwar nicht mit Güterpreisen argumentiert (sondern mit Realpreisen des Anteilscheins), wie die Budgetrestriktion (3.4) aber zeigt, läßt sich der Diskontfaktor $\delta_{t+1}$ als Gegenwartspreis des Konsums der folgenden Periode interpretieren.

Sei die mit einer Allokation einhergehende Güterpreisfolge mit $\left\{q_{t}\right\}_{t \in Z}$ bezeichnet, dann ist eine hinreichende Bedingung dafür, daß $\sum_{t} q_{t}<+\infty$ erfüllt ist, die Gültigkeit von $\delta_{t+1}<1, \forall t$ (es gilt $\delta_{t+1}=q_{t+1} / q_{t}, \delta_{t+2}=q_{t+2} / q_{t+1}$ usw.). ${ }^{3}$ $\mathrm{Da} ß$ der Diskontfaktor in jeder Periode kleiner als 1 ist, heißt nichts anderes als, daß die Güterpreise im Zeitablauf abnehmen. Es ist wegen $\delta_{t+1}=1 /\left(1+r_{t+1}\right)$ auch äquivalent dazu, daß der kurzfristige Zinssatz in jeder Periode positiv ist. Diese Schlußfolgerung ist mit den Ergebnissen in Cass (1972) kompatibel. ${ }^{4}$ In $(1-\varepsilon /(1-\delta), \varepsilon /(1-\delta))$ gilt $r=r^{l}=\varepsilon / p>0$. Somit ist das langfristige Gleichgewicht der Ökonomie intertemporal optimal.

damit unendlich viele Generationen und unendlich viele Güter). Shell (1971) führt die Möglichkeit suboptimaler Walras-Gleichgewichte auf die doppelte Unendlichkeit zurück; vgl. Shell (1971), S. 1005ff, und siehe auch Geanakoplos (1987a), S. 772ff. Zum Arrow-DebreuModell siehe Arrow und Debreu (1954) oder auch Geanakoplos (1987b).

' Siehe dazu Starrett (1972), S. 285, oder auch Azariadis (1993), S. 188.

${ }^{2}$ Vgl. Balasko und Shell (1980), S. 297.

${ }^{3} q_{t}$, ist der Güterpreis in Periode $t$ und $q_{t+1}$ der in Periode $t+1$. Die intertemporale Budgetrestriktion der Akteure lautet dann

$$
q_{t} \cdot c_{t}^{t}+q_{t+1} \cdot c_{t+1}^{t}=(1-\varepsilon) \cdot q_{t} \Leftrightarrow c_{t}^{t}+q_{t+1} / q_{t} \cdot c_{t+1}^{t}=1-\varepsilon .
$$

Wie man mit Hilfe der intertemporalen Budgetrestriktion (3.4) erkennt, gilt $q_{t+1} / q_{t}=\delta_{t+1}$.

${ }^{4}$ Siehe Cass (1972), S. 209ff. 
Zwischen der Verwendung von Zentralbankgeld (Außengeld) und der Optimalität einer Gleichgewichtsallokation besteht folgender Zusammenhang: Wie Cass et al. (1979) zeigen, kann Zentralbankgeld genau dann einen positiven Wert besitzen, wenn die reale Gleichgewichtsallokation (also die Gleichgewichtsallokation, die ohne die Verwendung von Zentralbankgeld resultiert) suboptimal ist. ${ }^{1}$ Somit besteht ein Zusammenhang zwischen der die Effizienz steigernden Wirkung von Zentralbankgeld und seiner positiven Bewertung. ${ }^{2}$ Dieser Zusammenhang rechtfertigt die Annahme, daß keine spekulative Preisblase auf den Anteilschein existieren kann, also $p_{t}=\varepsilon / r_{t+1}^{l}, \forall t$ gilt. Besitzt Zentralbankgeld einen positiven Wert, ist dieser eine spekulative Preisblase. ${ }^{3}$ Das langfristige Gleichgewicht der modellierten Ökonomie ist intertemporal optimal. Somit kann Zentralbankgeld im hier entwickelten Modell keine Verwendung und damit einen positiven Wert besitzen. Interpretiert man positiv bewertetes Zentralbankgeld als die spekulative Preisblase auf den Anteilschein, so kann diese nicht existieren. ${ }^{4}$

${ }^{1}$ Vgl. Cass et al. (1979), S. 49ff. Die Äquivalenz ist allerdings nicht mehr gegeben, wenn man Heterogenitäten bezüglich der Präferenzordnung und der Ausstattung der Akteure zuläßt; vgl. Cass et al. (1979), S. 53ff.

${ }^{2}$ Vgl. auch Wallace (1980), S. 52ff.

${ }^{3}$ Vgl. Bertochi und Wang (1995), S. 210ff. Zentralbankgeld besitzt keinen positiven Fundamentalwert. Dies folgt aus seinen beiden Eigenschaften Nichteinlösbarkeit und intrinsische Nutzlosigkeit; vgl. Wallace (1980), S. 49. Die Nichteinlösbarkeit besagt, daß der Emittent des Geldes (die Zentralbank) nicht verspricht das Geld gegen etwas anderes einzutauschen. Intrinsische Nutzlosigkeit besagt, daß Geld nicht um seiner selbst Willen nachgefragt wird. Es darf somit nicht Argument der Nutzenfunktion sein. Die Nichteinlösbarkeit ist allerdings nur dann gegeben, wenn es sich bei dem Geldsystem um keine Goldkernwährung handelt. Dort ist die Zentralbank verpflichtet, das von ihr emittierte Geld jederzeit in Gold einzulösen (Goldeinlösungspflicht). Siehe Issing (1992), S. 2ff, für die Darstellung alternativer Geldsysteme.

${ }^{4}$ Will man im vorliegenden Modell die Verwendung von Zentralbankgeld motivieren, so muß man auf eine der beiden Zusatzannahmen zurückgreifen, die aus dem RA-Modell bekannt sind. Dies ist zum einen die cash-in-advance Restriktion (auch Clower-Restriktion genannt). Die Idee, die der cash-in-advance Restriktion zugrunde liegt ist die, daß Geld Güter kauft und Güter Geld, Güter aber keine Güter; vgl. Clower (1967), S.5. Es wird somit auf die Tauschmittelfunktion des Geldes abgestellt (siehe dazu auch Grandmont und Younes (1973) und Lucas (1980)). Die zweite Annahme ist die, daß Geld ein Argument in der Nutzenfunktion ist. Hier wird die Annahme der intrinsischen Nutzlosigkeit von Geld fallengelassen (eine schöne Modellierung findet sich in Turnovsky (1995), S. 265ff und S. 356ff). Feenstra (1986) zeigt, daß die cash-in-advance Restriktion und die Annahme von Geld als Argument der Nutzenfunktion unter gewissen Voraussetzungen äquivalent sind (siehe auch Sargent (1987), Kap. 4 und 5, zur Darstellung beider Varianten). Beispiele für die Verbindung einer OLG-Struktur 


\subsubsection{Stabilität des langfristigen Gleichgewichts}

Es stellt sich nun die Frage nach der Stabilität des langfristigen Gleichgewichts. Hierbei geht es darum, was mit der Ökonomie passiert, wenn sie sich im langfristigen Gleichgewicht befindet und eine Abweichung stattfindet. $\mathrm{Zu}$ unterscheiden ist dabei zwischen zwei Stabilitätskonzepten. ${ }^{1}$ Ein stationäres Gleichgewicht ist stabil, wenn eine Folge nicht stationärer Gleichgewichte, die in seine Umgebung gerät, diese nicht mehr verläßt. Ein stationäres Gleichgewicht wird asymptotisch stabil genannt, wenn eine Folge nicht stationärer Gleichgewichte, die in seine Umgebung gerät, das stationäre Gleichgewicht asymptotisch erreicht. Die asymptotische Stabilität ist somit das stärkere Konzept. $\mathrm{Zu}$ unterscheiden ist noch die lokale und globale Stabilität. Die lokale Stabilität bezieht sich nur auf Folgen nicht stationärer Gleichgewichte, die in einer begrenzten Umgebung des stationären Gleichgewichts beginnen. Die globale Stabilität bezieht sich auf alle möglichen Folgen nicht stationärer Gleichgewichte. Ist ein Gleichgewicht nicht stabil, so wird es instabil genannt. ${ }^{2}$

Wie die Diskussion der Dynamik zeigte, führen alle Gleichgewichtspfade, die nicht unmittelbar im langfristigen Gleichgewicht $(1-\varepsilon /(1-\delta), \varepsilon /(1-\delta))$ beginnen, von diesem weg. Somit ist das langfristige Gleichgewicht instabil. ${ }^{3}$ Das langfristige Gleichgewicht der Ökonomie ist aber nicht in jedem Fall instabil.

mit einer cash-in-advance Restriktion finden sich unter anderem in Gagay et al. (1986) und Helpman und Razin (1987). Ein Beispiel für ein OLG-Modell mit Geld in der Nutzenfunktion bietet Helpman und Sadka (1979). Zu diesem Themenkomplex siehe auch Brock (1990).

I Vgl. Azariadis (1993), S. 12.

${ }^{2}$ Zur Stabilität siehe auch Day (1994), S. 88ff.

${ }^{3}$ Das langfristige Gleichgewicht ist der einzige durchfuihrbare Gleichgewichtspfad der Ökonomie und er ist instabil. Dies unterscheidet das hier entwickelte OLG-Modell von monetären OLG-Modellen. Im monetären OLG-Modell wird in Form von Zentralbankgeld gespart (vgl. Abschnitt 2.1). Kann Geld einen positiven Wert besitzen (Gale (1973) folgend, nennt man eine solche Ökonomie Samuelson-Ökonomie; vgl. Gale (1973), S. 20f), dann besitzt die Ökonomie genau zwei langfristige Gleichgewichte (dies ist das reale langfristige Gleichgewicht, hier findet zwischen den Generationen kein Handel statt und es wird nicht gespart, und das nominale langfristige Gleichgewicht, hier wird in Form von Geld gespart). In einer Samuelson-Ökonomie ist mindestens ein langfristiges Gleichgewicht stabil ( das reale langfristige Gleichgewicht ist immer stabil und das nominale langfristige Gleichgewicht unter gewissen Voraussetzungen) Vgl. dazu auch Wallace (1980), S. $53 \mathrm{ff}$. 
Die Stabilität hängt von der Krümmung der Tauschkurve im langfristigen Gleichgewicht ab. Dies führt zur Eigenschaft der Indeterminiertheit.

\subsection{Indeterminiertheit}

Die Eigenschaft eines Gleichgewichts, von anderen Gleichgewichten getrennt zu sein, wird Determiniertheit des Gleichgewichts genannt. ${ }^{1}$ Ein Gleichgewichtspfad ist indeterminiert, wenn in beliebig geringer Umgebung zu ihm ein weiterer Gleichgewichtspfad existiert, dessen Gleichgewichtsallokation beliebig nahe der Gleichgewichtsallokation des betrachteten Gleichgewichtspfads liegt. Wie aus der Gleichgewichtsdiskussion folgt, ist der Gleichgewichtspfad der betrachteten Ökonomie determiniert (es existiert genau ein Gleichgewichtspfad, und zwar das langfristige Gleichgewicht). Die Eigenschaft der Determiniertheit des oben beschriebenen Gleichgewichtspfads resultiert aus der Form der Tauschkurve und der Tatsache, daß die Anlage, in der gespart wird, also der Anteilschein an der Produktionstechnik, eine reale Anlage ist. Sie ist in dem Sinne real, daß sich mit ihrem Eigentum ein positiver Dividendenstrom verbindet, und ihr Fundamentalwert $\left(=\varepsilon / r_{t}^{l}\right)$ somit positiv ist. ${ }^{2}$ Es ist jedoch auch möglich, daß der Geichgewichtspfad der Ökonomie indeterminiert ist, wobei die Möglichkeit von der Form der Tauschkurve abhängt. ${ }^{3}$

\footnotetext{
' Vgl. Farmer (1993), S. 77ff. Der Entwicklungspfad einer Ökonomie ist indeterminiert, wenn sie unendlich viele sich durch die Allokation unterscheidende Walras-Gleichgewichte besitzt; vgl. auch Geanakoplos und Polemarchakis (1991), S. $1943 \mathrm{ff}$.

2 Ein Sparmedium ist eine nominale Anlage, wenn es keinen positiven Fundamentalwert besitzt. Diese Bedingung erfuillt Zentralbankgeld. Aufgrund seiner Eigenschaften der Nichteinlösbarkeit und der intrinsischen Nutzlosigkeit, ist positiv bewertetes Zentralbankgeld eine spekulative Preisblase; vgl. dazu Bertocchi und Wang (1995), S. 208ff. Zentralbankgeld kann nur dann einen positiven Wert besitzen, wenn die Akteure daran glauben. Verlieren die Akteure das Vertrauen in den Wert des Zentralbankgeldes, verliert es seine Kaufkraft. Keiner ist dann mehr bereit Zentralbankgeld gegen nutzenstiftende Güter zu tauschen. Der Fundamentalwert eines Vermögenstitels ist mit dem aus der Finanzierungsliteratur bekannten inneren Wert identisch; vgl. dazu z.B. Steiner und Bruns (1996), S. $186 \mathrm{f}$.

${ }^{3}$ Schon Calvo (1978) zeigt, daß auch im Fall einer realen Anlage Indeterminiertheit bei vollkommener Voraussicht möglich ist; vgl. Calvo (1978), S. $323 \mathrm{f}$.
} 
Wie oben erörtert, läßt sich die Auswirkung einer Zinsänderung auf den Konsum in der Jugend und den Konsum im Alter in den ISE und den EE aufspalten. Es zeigt sich, daß das Gleichgewicht $(1-\varepsilon /(1-\delta), \varepsilon /(1-\delta))$ dann indeterminiert und stabil ist, wenn der EE im Verhältnis zum ISE so stark ist, daß die Steigung der Tauschkurve im Bereich des langfristigen Gleichgewichts größer als 1 ist. Die Reduktion des Konsums, als Reaktion auf eine Erhöhung des Diskontfaktors, muß im Bereich des langfristigen Gleichgewichts in der Jugend höher sein als im Alter. Die hinreichende Bedingung für Indeterminiertheit lautet somit:

$$
\nabla_{\delta_{t+1}} c_{t}^{t}\left(\delta_{t+1}, 1-\varepsilon\right)<\nabla_{\delta_{t+1}} c_{t+1}^{t}\left(\delta_{t+1}, 1-\varepsilon\right){ }^{1}
$$

Darstellung 3.2 veranschaulicht diesen Zusammenhang. Die Herleitung des Gleichgewichtspfades erfolgt dabei entsprechend dem Fall der Bruttosubstitute in Darstellung 3.1. Es besteht nun jedoch das Problem, daß die Dynamik der Ökonomie nicht mehr eindeutig bestimmt ist. ${ }^{2}$ Nun gibt es zu einem gegebenen Diskontfaktor $\delta_{t+1}$ zwei Diskontfaktoren $\delta_{t+2}$ der nächsten Periode, die den Gütermarkt in Periode $t+1$ räumen (prinzipiell ist es möglich, von der Diagonalen aus nach links oder nach rechts zu loten). Beschränkt man sich auf die Beschreibung der lokalen Dynamik, so ist das in Darstellung 3.2 gezeigte Vorgehen adäquat. ${ }^{3}$ Eine in der Nähe des langfristigen Gleichgewichts beginnende Folge nicht stationärer Gleichgewichte konvergiert hin zu diesem. Der Diskontfaktor oszilliert hierbei mit sinkender Amplitude um den Diskontfaktor des langfristigen Gleichgewichts, bis er diesen schließlich nach abzählbar vielen Perioden erreicht. Hierbei existiert in beliebig geringer Umgebung zu jedem Gleichgewichtspfad ein weiterer Pfad, der ebenfalls konvergiert. Das langfristige

${ }^{1}$ Dies ist die gleiche Bedingung, die auch die Stabilität des nominalen langfristigen Gleichgewichts einer monetären OLG-Ökonomie gewährleistet; vgl. dazu Grandmond (1985), S. 1018. Im nominalen langfristigen Gleichgewicht entspricht der Zinssatz der Rate des Bevölkerungswachstums; siehe z.B. Blanchard und Fischer (1989), S. 159.

${ }^{2}$ Vgl. Benhabib und Day (1982), S. 42f.

${ }^{3}$ Dies entspricht dem Vorgehen von Grandmont (1985) im monetären Modell; vgl Grandmont (1985), S. 1005. Die Rückwärtsdynamik (von der Gegenwart in die Vergangenheit) ist weiterhin eindeutig determiniert. Die Vorwärtsdynamik (die Dynamik, wie sie hier verstanden wird) wird lokal approximiert, indem man die Rückwärtsdynamik beschreibende Differenzengleichung im langfristigen Gleichgewicht linearisiert und dann invertiert. 
Gleichgewicht ist somit lokal asymptotisch stabil und indeterminiert. Technisch gesprochen handelt es sich bei ihm um eine Senke. Die Eigenschaft, daß die Güter aufeinanderfolgender Perioden Bruttokomplemente sind, ist für die lokale asymptotische Stabilität und Indeterminiertheit des langfristigen Gleichgewichts bei vollkommener Voraussicht somit notwendig, jedoch nicht hinreichend: Der EE muß so stark sein, daß die Tauschkurve im Bereich des langfristigen Gleichgewicht eine Steigung aufweist, die größer als 1 ist. ${ }^{1}$

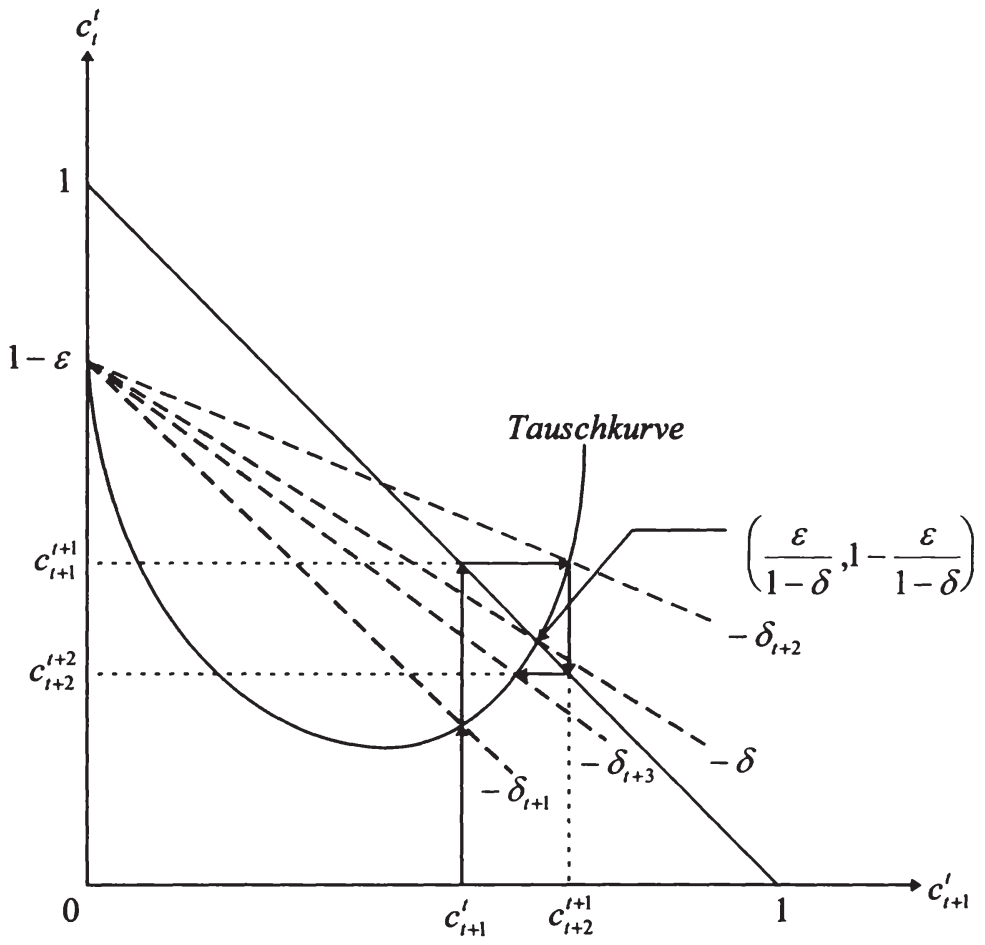

Darst. 3.2: Indeterminiertheit

${ }^{1}$ Vgl. auch Balasko und Ghiglino (1995), S. 570ff. 
Es müssen also zwei Fälle unterschieden werden. Zum einen der Fall des determinierten instabilen langfristigen Gleichgewichts, das gleichzeitig den einzigen Gleichgewichtspfad der Ökonomie darstellt, und zum andern der Fall des lokal asymptotisch stabilen langfristigen Gleichgewichts, in dem der Gleichgewichtspfad der Ökonomie indeterminiert ist. Dies führt zum Konzept der Regularität eines Modells. ${ }^{1}$

Ein Modell wird regulär genannt, wenn es genügend Restriktionen besitzt, um einen eindeutigen Pfad zu determinieren, der hin zum langfristigen Gleichgewicht konvergiert. Ein Modell wird irregulär genannt, wenn es nicht genügend Restriktionen aufweist, um die Anfangsbedingungen eines Pfades eindeutig zu determinieren. Somit ist das vorliegende Modell irregulär oder regulär, je nachdem, ob das langfristige Gleichgewicht lokal asymptotisch stabil oder instabil ist. $^{2}$ Dies unterscheidet das OLG-Modell der Arbeit von anderen OLG-Modellen. Monetäre OLG-Modelle, in denen die modellierten Ökonomien vom Samuelson-Typ sind (Zentralbankgeld kann in solchen Ökonomien aufgrund seiner Wertaufbewahrungsfunktion einen positiven Wert besitzen) und OLG-Modelle vom skalaren Diamond-Typ (der Kapitalstock zu Beginn einer Periode ist gleich der Ersparnis der vorherigen Periode, und die Kapitalintensität ist die einzige Zustandsvariable) sind irregulär. ${ }^{3}$ OLG-Modelle, in denen die model-

' Vgl. dazu Farmer (1993), S. 45ff.

${ }^{2}$ Im Fall des instabilen langfristigen Gleichgewichts wird der Gleichgewichtspfad eindeutig determiniert. Er muß im langfristigen Gleichgewicht beginnen, ansonsten verläßt er nach abzählbar vielen Perioden den durchführbaren Bereich. Im Fall des lokal asymptotisch stabilen langfristigen Gleichgewichts konvergieren alle Pfade, die in der Umgebung des langfristigen Gleichgewichts beginnen, hin zu diesen. Das Modell besitzt nicht genügend Restriktionen um die Anfangsbedingungen des Gleichgewichtspfades eindeutig zu determinieren (das langfristige Gleichgewicht ist eine Senke).

${ }^{3}$ Im monetären OLG-Modell wird ein Gleichgewichtspfad durch das anfängliche Preisniveau determiniert. Das Modell besitzt nicht genügend Restriktionen dieses festzulegen. Arbiträr fixierte anfängliche Preisniveaus führen zu sich durch die Allokation unterscheidende Gleichgewichtspfade, und es gibt unendlich viele von diesen (vgl. z.B. Geanakoplos und Polemarchakis (1986), S. 757ff). In der skalaren Version des Modells von Diamond (1965), führen alternative anfängliche Niveaus des Pro-Kopf-Konsums, bei einem gegebenen anfänglichen Niveau der Kapitalintensität, zu sich durch die Allokation unterscheidende Gleichgewichtspfade. Das Modell besitzt nicht genügend Restriktionen um einen eindeutigen Pfad zu determinieren. Eine schöne Darstellung des Diamond-Modells bietet z.B. Romer (1996), S. $72 \mathrm{ff}$. 
lierten Ökonomien vom klassischen Typ sind (Zentralbankgeld besitzt in solchen Ökonomien keinen positiven Wert) und OLG-Modelle vom planaren Diamond-Typ (hier sind die Kapitalintensität und die nationale Pro-Kopf-Verschuldung die Zustandsvariablen) sind regulär. ${ }^{1}$ Man kann sagen, daß genau dann, wenn das langfristige Gleichgewicht eine Senke ist, das Modell irregulär ist.

Wie die Diskussion der Determiniertheit zeigt, ist es sinnvoll den regulären Fall zu betrachten, will man die dynamischen Wirkungen von politischen Maßnahmen analysieren. Dieses Vorgehen wird weiter unten gewählt, wenn die Auswirkungen von staatlicher Schuldenpolitik und Außenhandel untersucht werden. Hierbei wird methodisch so vorgegangen, daß sich das ökonomische System im langfristigen Gleichgewicht befindet, und in einer Periode ein unerwarteter Strukturbruch stattfindet (die von den Akteuren nicht antizipierte Politikmaßnahme stellt hierbei den Strukturbruch dar). Würde man den irregulären Fall betrachten, so wäre die auf den Strukturbruch folgende Dynamik indeterminiert, da in diesem Fall unendlich viele Pfade nicht stationärer Gleichgewichte existieren, die hin zum neuen langfristigen Gleichgewicht konvergieren. Im regulären Fall muß sich das ökonomische System in der auf den Strukturbruch folgenden Periode im neuen langfristigen Gleichgewicht befinden (alle anderen Pfade verlassen nach abzählbar vielen Perioden den durchführbaren Bereich). Nur in diesem Fall sind gehaltvolle Aussagen über die aus den Politikmaßnahmen resultierenden Wirkungen auf die Allokation, den Zins und die Wohlfahrt möglich.

Zur Unterscheidung zwischen skalarem Diamond-Modell und planarem Diamond-Modell siehe Azariadis (1993), S. 75ff.

${ }^{1}$ So wie im regulären Fall im Modell der vorliegenden Arbeit, sind in einer klassischen Ökonomie alle Pfade, die nicht im realen langfristigen Gleichgewicht beginnen, nach abzählbar vielen Perioden nicht mehr durchführbar. Somit besitzt eine klassische Ökonomie genau einen Gleichgewichtspfad. Sie muß sich von Anfang an im realen langfristigen Gleichgewicht befinden (dieses ist determiniert und instabil); vgl. dazu Gale (1973), S. 22ff. Im planaren Diamond-Modell ist das langfristige Gleichgewicht (mit positiver nationaler Pro-Kopf-Verschuldung) ein Sattelpunkt; vgl. Azariadis (1993), S. 84. Zur Sattelpunkteigenschaft siehe. z.B. Takayama (1994), S. 103ff. 


\subsubsection{Die Zinsdynamik}

Im Folgenden soll der Zinspfad untersucht werden, der resultiert, wenn die Ökonomie sich in der Nähe des langfristigen Gleichgewichts befindet und hin zu diesem konvergiert (betrachtet wird also der irreguläre Fall). Hierbei interessiert die Zinsstruktur und die Zinsspreizung, also die Differenz zwischen dem kurzfristigen Zinssatz und dem langfristigen Zinssatz. Wie man aus Darstellung 3.2 ersieht, oszilliert der Diskontfaktor mit einer sinkenden Amplitude um den Diskontfaktor des langfristigen Gleichgewichts, bis er diesen nach abzählbar vielen Perioden erreicht. Betrachtet wird der Fall, indem die Diskontfaktoren der Perioden $t, t+2, t+4$ usw. niedriger als $\delta$ sind bzw. die Diskontfaktoren der Perioden $t+1, t+3, t+5$ usw. höher als $\delta$. Dies ist äquivalent dazu, daß die kurzfristigen Zinssätze der Perioden $t, t+2, t+4$ usw. höher als $r$ sind bzw. die kurzfristigen Zinssätze der Perioden $t+1, t+3, t+5$ usw. niedriger als $r$. Wie aus Beziehung (3.1) folgt ist dies äquivalent dazu, daß der Preis des Anteilscheins in den Perioden $t, t+2, t+4$ usw. höher als $p$ ist bzw. in den Perioden $t+1, t+3, t+5$ usw. niedriger als $p$. Somit müssen die Generationen $t, t+2$, $t+4$ usw. einen Kapitalverlust hinnehmen bzw. profitieren die Generationen $t+1, t+3, t+5$ usw. von einem Kapitalgewinn (es gilt $p_{t}>p_{t+1}, p_{t+2}>p_{t+3}, p_{t+4}$ $>p_{t+5}$ usw. bzW. $p_{t+1}<p_{t+2}, p_{t+3}<p_{t+4}, p_{t+5}<p_{t+6}$ usw.).

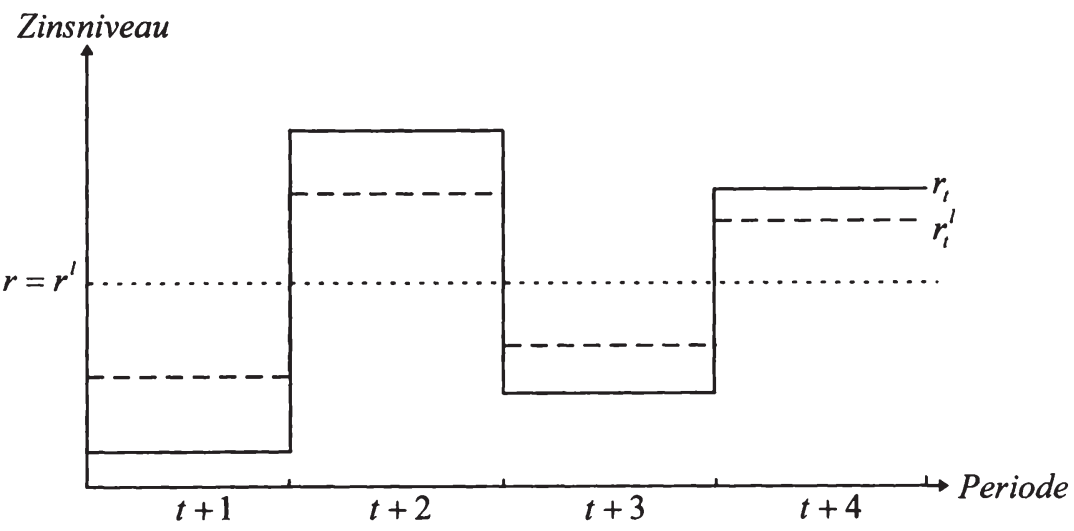

Darst. 3.3: Zinsdynamik 
Darstellung 3.3 veranschaulicht den Zinspfad. Wie aus der Arbitragebedingung (3.2) folgt, wechseln sich auf dem Pfad hin zum langfristigen Gleichgewicht Perioden mit normaler Zinsstruktur und Perioden mit inverser Zinsstruktur ab. Dies ist auch verständlich, da sich Perioden mit Kapitalverlust und Perioden mit Kapitalgewinn abwechseln. Die Zinsspreizung nimmt im Zeitablauf ab, da die Preisfluktuationen des Anteilscheins im Zeitablauf abflachend sind (vgl. Darstellung 3.2). Dasselbe gilt für die Variabilität der Zinssätze im Zeitablauf. Nach abzählbar vielen Perioden erreichen die Zinssätze ihr langfristiges Niveau. Es sei noch bemerkt, daß die Fluktuation des langfristigen Zinssatzes immer geringer ist als die des kurzfristigen Zinssatzes.

\subsubsection{Zyklische Gleichgewichte}

Eng verbunden mit der Indeterminiertheit von $(1-\varepsilon /(1-\delta), \varepsilon /(1-\delta))$ ist die Möglichkeit der Existenz von zyklischen Gleichgewichten. ${ }^{1}$ Die Ökonomie befindet sich in einem k-periodischen Zyklus, wenn die Konsumvektoren genau alle $\mathbf{k}$ Perioden wiederkehren. ${ }^{2}$ Betrachtet sei der Fall des zweiperiodischen $\mathrm{Zy}$ klus. Es wird nun gezeigt, daß die lokale asymptotische Stabilität und Indeterminiertheit von $(1-\varepsilon /(1-\delta), \varepsilon /(1-\delta))$ die Existenz eines zweiperiodischen $\mathrm{Zy}$ klus impliziert. ${ }^{3}$

Die Existenz des zyklischen Gleichgewichts läßt sich innerhalb des Tauschkurvendiagramms geometrisch herleiten. ${ }^{4}$ Sei hierfür Darstellung 3.4 betrachtet. Man erhält das zyklische Gleichgewicht durch Spiegelung der Tauschkurve an der Diagonalen. Der Schnittpunkt der Tauschkurve mit ihrem Spiegelbild, der

${ }^{1}$ Diese sind deshalb von besonderem Interesse, da die im Modell erzeugten Zyklen Beispiele für rein modellendogen begründete, in einer deterministischen Umwelt auftretende, Konjunkturzyklen sind. Siehe dazu auch Grandmont $(1985,1993)$, Boldrin und Woodford (1990), S. $210 \mathrm{ff}$, und Balasko und Ghiglino (1995). Zur Stabilitätsdiskussion solcher Zyklen sei auf Grandmont und Laroque (1986) verwiesen.

${ }^{2}$ Siehe dazu auch Baumol und Benhabib (1989), S. $81 \mathrm{ff}$.

${ }^{3}$ Dies ist die gleiche Bedingung, die auch die Existenz eines zweiperiodischen Zyklus im monetären OLG-Modell impliziert; vgl. Grandmont (1985), S. $1018 \mathrm{f}$.

${ }^{4}$ Vgl. z.B. Azariadis und Guesnerie (1986), S. 727f, oder Kehoe (1989), S. 378. 
auf der Diagonalen liegt, gibt das nicht eingezeichnete langfristige Gleichgewicht $(1-\varepsilon /(1-\delta), \varepsilon /(1-\delta))$ mit dem über die Zeit konstanten Diskontfaktor $\delta$ an. Die Schnittpunkte, die nicht auf der Diagonalen liegen, treten wegen der Symmetrie paarweise auf. Sie korrespondieren mit dem zyklischen Gleichgewicht. Der zweiperiodische Zyklus zeichnet sich durch die genau alle zwei Perioden wiederkehrenden Konsumvektoren $A$ und $B$ der Generationen und ebenfalls genau alle zwei Perioden wiederkehrende Diskontfaktoren $\delta_{A}$ und $\delta_{B}$ aus.

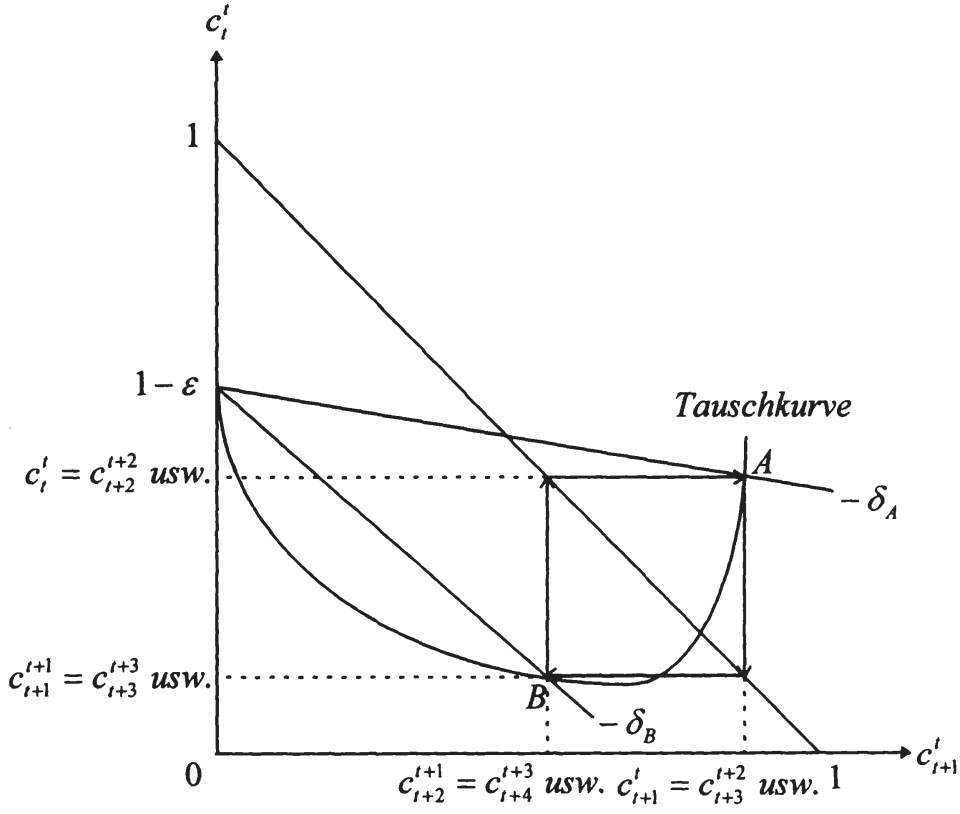

Darst. 3.4: Zyklisches Gleichgewicht

Der Zinspfad des zweiperiodischen Zyklus läßt sich wie folgt eruieren. Mit $\delta_{A}$ $=p_{B} /\left(\varepsilon+p_{A}\right)=1 /\left(1+r_{A}\right)$ und $\delta_{B}=p_{A} /\left(\varepsilon+p_{B}\right)=1 /\left(1+r_{B}\right)$ sowie $r_{A}^{\prime}=\varepsilon / p_{B}$ und $r_{B}^{l}=\varepsilon / p_{A}$ erhält man analog zu (3.2) folgende Arbitragebedingungen: 


$$
\begin{aligned}
& r_{A}=\frac{\varepsilon}{p_{B}}+\frac{p_{A}-p_{B}}{p_{B}}=r_{A}^{l}-\frac{r_{B}^{l}-r_{A}^{l}}{r_{B}^{l}}, \\
& r_{B}=\frac{\varepsilon}{p_{A}}+\frac{p_{B}-p_{A}}{p_{A}}=r_{B}^{l}-\frac{r_{A}^{l}-r_{B}^{l}}{r_{A}^{l}} .
\end{aligned}
$$

Die Generationen, deren Konsumvektor $A$ ist (dies sind die Generationen $t$, $t+2, t+4$ usw.) erzielen eine höhere Rendite aus ihrer Ersparnis, als die Generationen, deren Konsumvektor $B$ ist (dies sind die Generationen $t+1, t+3, t+5$ usw.). Zum einen ist der Ertrag in $A$ höher als in $B\left(\varepsilon / p_{B}>\varepsilon / p_{A}\right.$ da $\left.p_{B}<p_{A}\right)$, zum anderen findet in $A$ ein Kapitalgewinn statt und in $B$ ein Kapitalverlust $\left(\left(p_{A}-p_{B}\right) / p_{B}>0\right.$ und $\left(p_{B}-p_{A}\right) / p_{A}<0$ da $\left.p_{B}<p_{A}\right)$. Die Zinsdynamik im zweiperiodischen Zyklus sieht wie folgt aus:

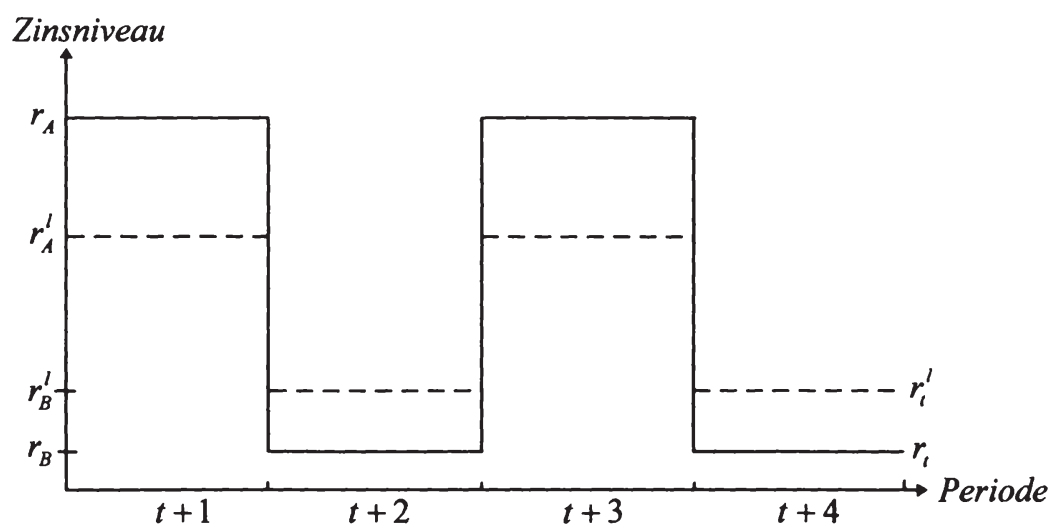

Darst. 3.5: Zinsdynamik im zweiperiodischen Zyklus

Darstellung 3.5 veranschaulicht die Zinsdynamik im zweiperiodischen Zyklus. Man erkennt, daß die Zinssätze mit konstanter Amplitude oszillieren, wobei die Volatilität des langfristigen Zinssatzes geringer ist als die Volatilität des kurzfristigen Zinssatzes. Dies erzeugt sich abwechselnde Perioden mit normaler und 
inverser Zinsstruktur. Die Zinsspreizung ist im Gleichgewicht $A$ größer als im Gleichgewicht $B$, denn wegen des Basiseffekts gilt:

$$
\left|\frac{r_{B}^{l}-r_{A}^{l}}{r_{B}^{l}}\right|>\frac{r_{A}^{l}-r_{B}^{l}}{r_{A}^{l}} .
$$

Es ist offensichtlich, daß im zweiperiodischen Zyklus jede zweite Generation gegenüber dem langfristigen Gleichgewicht besser bzw. schlechter gestellt wird. Der Konsumvektor $B$ liegt innerhalb der Budgetmenge der Akteure im langfristigen Gleichgewicht, der Konsumvektor $A$ liegt außerhalb. ${ }^{1}$ Dies führt zur Frage der Optimalität der diskutierten Gleichgewichte. Im zweiperiodischen Zyklus alterniert der Zinssatz zwischen zwei Werten, die beide positiv sind. Wie aus Abschnitt 3.1.2 folgt, ist dies hinreichend dafür, daß das zyklische Gleichgewicht intertemporal optimal ist.

${ }^{1}$ Die Verzinsung der Ersparnis der Akteure $t, t+2$ usw. ist im zweiperiodischen Zyklus gegenüber dem langfristigen Gleichgewicht höher $\left(\delta_{A}^{-1}>\delta^{-1}\right)$. Thr Konsumprogramm liegt in $A$. Die Verzinsung der Ersparnis der Akteure $t+1, t+3$ usw. ist im zweiperiodischen Zyklus gegenüber dem langfristigen Gleichgewicht geringer $\left(\delta_{B}^{-1}<\delta^{-1}\right)$. Ihr Konsumprogramm liegt in $B$. Anders als bei einem zweiperiodischen Zyklus in einer stationären monetären OLGÖkonomie, gilt in der hier diskutierten Ökonomie $\delta_{A} \neq 1 / \delta_{B}$. Dies resultiert aus dem Umstand, daß im stationären monetären OLG-Modell $\delta=1$ gilt und hier $\delta<1$. 


\section{Die Auswirkungen von Staatsverschuldung}

Sei die Existenz eines öffentlichen Sektors innerhalb der Ökonomie angenommen. Es findet kein öffentlicher Verbrauch von Gütern statt. Die einzige Aktivität der Regierung besteht darin, junge Akteure zu besteuern bzw. ihnen Transfers zukommen zu lassen. Der Staat betreibt also eine reine Umverteilungspolitik. Transfers werden hierbei durch den Verkauf von Staatsschuldverschreibungen (Staatsanleihen) finanziert. Der aus den emittierten Staatsanleihen resultierende Zinsendienst wird durch die Besteuerung des Arbeitseinkommens zukünftiger Generationen bestritten. ${ }^{1}$

Sei $\tau_{t}<0$ der Transfer an den jungen Akteur $t$ bzw. $\tau_{t}>0$ die Steuer auf sein Arbeitseinkommen. Ein Transfer ist hierbei als Realtransfer zu verstehen. ${ }^{2}$ Sei $b$, die Anzahl der sich in Periode $t$ im Umlauf befindenden Anleihen. Es befinden sich nun zwei Anlageinstrumente im Umlauf. Das ist zum einen der Anteilschein an der Produktionstechnik, und das sind zum anderen die Anleihen. Beide Vermögensklassen seien vollkommene Substitute im Portfolio der Anleger. Hierfür ist es notwendig, daß eine Anleihe mit einem unendlichen Strom von Couponzahlungen in Höhe von $\varepsilon$ Gütereinheiten pro Periode verbunden ist. Bei den Anleihen handelt es sich somit um ewige Anleihen. $p_{t}$ ist jetzt sowohl der Preis des Anteilscheins als auch der Preis einer Anleihe (der Anteilschein bzw. die Anleihen werden ex Dividende bzw. ex Coupon gehandelt). Die Budgetrestriktion des Staates in Periode $t$ lautet: Anleiheemission

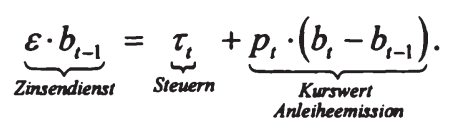

\footnotetext{
' Eine entsprechende Modellierung des öffentlichen Sektors findet sich auch bei Fried und Howitt (1988).

${ }^{2}$ Hierfür geht man davon aus, daß die in Periode $t$ emittierten Staatsanleihen von Akteur $t$ auf dem Kapitalmarkt dieser Periode erworben werden und ihm der Kaufpreis unmittelbar als Realtransfer zukommt. Dem Akteur $t$ ist bei seiner Sparentscheidung die Höhe des ihm zukommenden Transfers bekannt. Tatsächlich besteht der Transfer somit in den emittierten Anleihen, die der begünstigte Akteur in seiner Jugend faktisch kostenlos erhält. Im Alter fließen ihm dann die Zinszahlung und der Verkaufserlös zu.
} 
Die Staatsausgaben bestehen aus dem Zinsendienst und gegebenenfalls einem Transfer $\left(\tau_{t}<0\right)$. Sie müssen durch Steuern $\left(\tau_{t}>0\right)$ und gegebenenfalls durch die Emission von Anleihen finanziert werden.

Das Verhältnis des gegenwärtigen Wertes eines Vermögenstitels (sei es der Anteilschein an der Produktionstechnik oder eine Staatsanleihe) zu seinem Wert in der folgenden Periode ist gleich dem einperiodigen Diskontfaktor. Beziehung (3.1) besitzt somit weiterhin Gültigkeit. Die Existenz des Staates verändert das intertemporale Nutzenmaximierungsproblem der Akteure wie folgt:

$$
u\left(c_{t}^{t}, c_{t+1}^{t}\right) \rightarrow \underset{\substack{c_{t}^{t}, c_{t+1}^{t} \\ c^{\prime}}}{ }
$$

unter der $N B$

$$
\begin{aligned}
c_{t}^{t}+s^{t} & =1-\varepsilon-\tau_{t} & & \text { (Budgetrestriktion Jugend) } \\
c_{t+1}^{t} & =\delta_{t+1}^{-1} \cdot s^{t} & & \text { (Budgetrestriktion Alter) }
\end{aligned}
$$

Vergleicht man das Optimierungsproblem mit dem durch (3.3) gegebenen, so erkennt man, daß sich beide nur durch die Jugendbudgetrestriktion unterscheiden. Das verfügbare Einkommen eines Akteurs wird durch die Besteuerung seines Arbeitseinkommens reduziert oder durch den ihm zukommenden Transfer erhöht. Die Budgetrestriktion der Jugend und die des Alters lassen sich wieder in eine intertemporale Budgetrestriktion transformieren:

$$
c_{t}^{t}+\delta_{t+1} \cdot c_{t+1}^{t}=1-\varepsilon-\tau_{t}
$$

Es ist offensichtlich, daß die Bedingungen erster Ordnung der Nutzenmaximierung zur selben Optimalitätsbedingung, wie im Fall ohne aktiven Staat, führt. Die Optimalitätsbedingung (3.9) besitzt somit weiterhin Gültigkeit. Das optimale Konsumprogramm zeichnet sich dadurch aus, daß die Grenzrate der Substitution im Konsum mit der Verzinsung der Ersparnis (= die ökonomische Substitutionsrate) übereinstimmt. Aus der Optimierung folgen die Nachfragefunktionen der jungen und der alten Akteure, sowie ihre Sparfunktion. Sie sind wieder eine Funktion des Diskontfaktors und des verfügbaren Einkommens: $c_{t}^{t}=$ $c_{t}^{t}\left(\delta_{t+1}, 1-\varepsilon-\tau_{t}\right), c_{t+1}^{t}=c_{t+1}^{t}\left(\delta_{t+1}, 1-\varepsilon-\tau_{t}\right)$ und $s^{t}=s^{t}\left(\delta_{t+1}, 1-\varepsilon-\tau_{t}\right)$. 
Am Kapitalmarkt treten nun zwei Anbieter von Vermögenstiteln bzw. zwei Nachfrager nach Kapital auf. Das ist zum einen der alte Akteur, der den von ihm gehaltenen Anteilschein und die von ihm gehaltenen Staatsanleihen anbietet. Der zweite Anbieter ist der Staat, der gegebenenfalls neue Anleihen emittiert. Die Gleichgewichtsbedingung des Kapitalmarktes lautet somit:

$$
s^{t}\left(\delta_{t+1}, 1-\varepsilon-\tau_{t}\right)=\underbrace{p_{t} \cdot\left(1+b_{t-1}\right)}_{\text {Angebot alter Atteur }}+\underbrace{p_{t} \cdot\left(b_{t}-b_{t-1}\right)}_{\text {Angebot Staat }}=p_{t} \cdot\left(1+b_{t}\right), \forall t .
$$

Die Ersparnis des jungen Akteurs (die Differenz zwischen verfügbarem Einkommen und Konsum) muß gleich dem Kurswert der ihm angebotenen Vermögenstitel sein, damit der Kapitalmarkt geräumt wird. Da der Staat keine Güter konsumiert, bleibt Gleichung (3.11) weiterhin die Gleichgewichtsbedingung für den Gütermarkt.

Ein Gleichgewichtspfad der Ökonomie läßt sich also wie folgt beschreiben:

Eine Folge $\left\{p_{t}, \tau_{t}, b_{t},\left(c_{t}^{t}, c_{t+1}^{t}\right)\right\}_{t \in \mathcal{Z}}$ beschreibt ein Walras-Gleichgewicht der Ökonomie bei vollkommener Voraussicht, wenn $\left\{\tau_{t}, b_{t}\right\}_{1 \in Z}$ die staatliche Budgetrestriktion (4.1) erfüllt, $\left\{\left(c_{t}^{t}, c_{t+1}^{t}\right)\right\}_{t \in Z}$ der Optimalitätsbdingung (3.9) genügt, und sowohl der Gütermarkt als auch der Kapitalmarkt geräumt werden, also (3.11) und (4.4) gelten.

\subsection{Die langfristigen Auswirkungen der Staatsverschuldung}

Ein langfristiges Gleichgewicht der Ökonomie zeichnet sich dadurch aus, daß die Anzahl der sich im Umlauf befindenden Anleihen über die Zeit konstant ist, und der Staat den Zinsendienst durch eine über die Zeit konstante Besteuerung des Arbeitseinkommens bestreitet. Aus der staatlichen Budgetrestriktion folgt $\tau=\varepsilon \cdot b$. Der Preis der Vermögenstitel und der Diskontfaktor sind im langfristi- 
gen Gleichgewicht konstant. Aus Beziehung (3.1) folgt wieder $p=\delta \cdot \varepsilon /(1-\delta)$. Der langfristige Konsumvektor der Generationen ist damit:

$$
\begin{aligned}
c_{t}^{t}(\delta, 1-\varepsilon-\tau) & =\underbrace{1-\varepsilon-\tau}_{\begin{array}{c}
\text { verfiubares } \\
\text { Eintommen }
\end{array}}-\underbrace{p \cdot(1+b)}_{\begin{array}{c}
\text { Kurswert } \\
\text { Vermogenstitel }
\end{array}} \\
& =1-\varepsilon-\varepsilon \cdot b-\frac{\delta \cdot \varepsilon}{1-\delta} \cdot(1+b)=1-\frac{\varepsilon+\varepsilon \cdot b}{1-\delta}, \\
c_{t+1}^{t}(\delta, 1-\varepsilon-\tau) & =\underbrace{\varepsilon \cdot(1+b)}_{\begin{array}{c}
\text { Dividende } \\
\text { Coupons }
\end{array}}+\underbrace{p \cdot(1+b)}_{\begin{array}{c}
\text { Kurswert } \\
\text { Vermögenstitiel }
\end{array}} \\
& =\varepsilon \cdot(1+b)+\frac{\delta \cdot \varepsilon}{1-\delta} \cdot(1+b)=\frac{\varepsilon+\varepsilon \cdot b}{1-\delta} .
\end{aligned}
$$

Der Jugendkonsum wird durch die Differenz zwischen dem verfügbaren Einkommen der Akteure und dem Kurswert des Vermögenstitelbestandes gegeben. Der Alterskonsum ist gleich der Summe aus den Coupon- und Dividendenzahlung und dem Kurswert des Vermögenstitelbestandes. Das langfristige Gleichgewicht der Ökonomie wird somit durch den stationären Konsumvektor $(1-(\varepsilon+\varepsilon \cdot b) /(1-\delta),(\varepsilon+\varepsilon \cdot b) /(1-\delta))$ charakterisiert. Da der Gütermarkt und der Kapitalmarkt weiterhin die zwei einzigen Märkte sind, läßt sich das langfristige Gleichgewicht der Ökonomie mit Staat durch alleinige Betrachtung des Gütermarktes analysieren (Walras-Gesetz).

Darstellung 4.1 zeigt, wie sich die Verschuldung des Staates auf das langfristige Gleichgewicht der Ökonomie auswirkt. Situation $a$ beschreibt den Fall der Ökonomie mit unverschuldetem Staat und Situation $b$ den Fall einer Ökonomie mit verschuldetem Staat. Im Fall des verschuldeten Staates sinkt das verfügbare Einkommen der jungen Akteure durch die notwendige Besteuerung des Arbeitseinkommens. Der Diskontfaktor ist in Situation $b$ niedriger als in Situation $a$, was bedeutet, daß die Staatsverschuldung den Zinssatz erhöht. Dies folgt aus der Normalgut-Annahme. Diese bedingt, daß je niedriger das Verhältnis von Konsum in der Jugend und Konsum im Alter wird, desto geringer die Steigung der Indifferenzkurve im gleichgewichtigen Konsumpunkt ist. Dieser Zusammenhang läßt sich durch ein geometrisches Experiment leicht zeigen: Ver- 
schiebt man die Budgetgerade parallel nach unten (= Reduktion des verfügbaren Einkommens), erzeugt dies eine reduzierte Nachfrage in der Jugend und im Alter (Normalgut-Annahme). Der neue gleichgewichtige Konsumvektor geht jedoch mit einem erhöhten Konsum im Alter einher (das Gleichgewicht wandert auf der Diagonalen nach rechts unten). Damit dies möglich ist, muß der Zinssatz steigen bzw. der Diskontfaktor sinken, denn es gilt $\partial c_{t+1}^{t} / \partial \delta_{t+1}<0$ (unabhängig davon, ob die Güter Bruttosubstitute oder Bruttokomplemente sind).

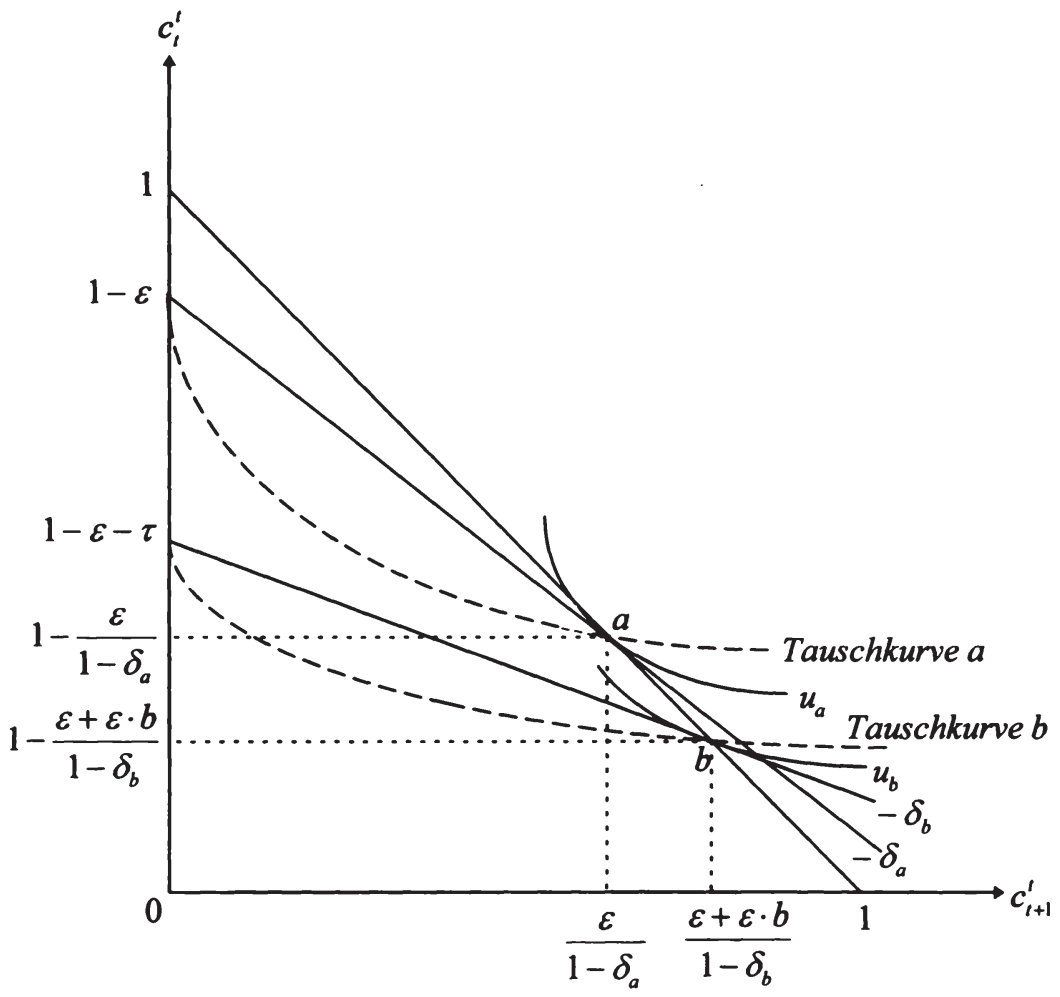

Darst. 4.1: Auswirkungen von Staatsverschuldung 
Aus $p=\delta \cdot \varepsilon /(1-\delta)$ folgt, daß der Preis der Vermögenstitel durch die Staatsverschuldung sinkt. In einer Ökonomie mit einem verschuldeten Staat ist der Konsum der jungen Akteure niedriger und der Konsum der alten Akteure höher als in der Ökonomie ohne Staatsschuld. Daß dies der Fall ist erkennt man aus den Gleichungen für den stationären Konsumvektor. Zum einen erhöht sich mit der Staatsverschuldung die Anzahl der sich im Umlauf befindenden Vermögenstitel, zum anderen reduziert die Staatsverschuldung den Diskontfaktor.

Man erkennt, daß die Staatsverschuldung die Wohlfahrt der Akteure reduziert: $u_{a}$ ist das erreichbare Nutzenniveau in der Ökonomie mit positiver Staatsschuld und $u_{b}$ das in der Ökonomie ohne Staatsschuld. $\mathrm{Da} \beta u_{a}>u_{b}$ gelten muß, läßt sich mit Hilfe der Theorie der offenbarten Präferenz leicht zeigen. ${ }^{1}$ Ein Konsumplan $a$ wird gegenüber einem Konsumplan $b$ offenbart präferiert, wenn beide Konsumpläne durchführbar sind und $a$ gewählt wird. Wird $a$ gegenüber $b$ offenbart präferiert, dann kann es nicht sein, daß $b$ gegenüber $a$ offenbart präferiert wird (schwaches Axiom der offenbarten Präferenz). Wird $a$ gegenüber $b$ offenbart präferiert impliziert dies, daß $a$ mindestens so viel Nutzen stiftet wie $b .^{2}$ In Darstellung 4.1 ist $a$ der langfristige Konsumvektor in der Ökonomie ohne Staatsschuld und $b$ der langfristige Konsumvektor in der Ökonomie mit positiver Staatsschuld. Wie man erkennt, liegt $b$ innerhalb der Budgetmenge der Akteure der Ökonomie ohne Staatsschuld. Somit wäre $b$ für die Akteure der Ökonomie ohne Staatsschuld nicht nur durchführbar, sondern der Wert von $b$ ist sogar geringer als der Wert von $a$. Damit wird $a$ gegenüber $b$ sogar streng offenbart präferiert. Dies impliziert, daß $a$ echt nutzenstiftender ist als $b .{ }^{3}$ Es gilt also $u_{a}>u_{b}$. Je höher die Staatsverschuldung ist, desto geringer wird das von den Akteuren erreichbare Nutzenniveau, da der optimale Konsumvektor der Akteure einer Ökonomie mit höherer Staatsverschuldung immer innerhalb der Budgetmenge der Akteure einer Ökonomie mit niedrigerer Staatsverschuldung liegt. Mit steigender Staatsverschuldung sinkt der Jugendkonsum und erhöht sich der Alterskonsum (der langfristige Konsumvektor wandert mit

\footnotetext{
${ }^{1}$ Im Anhang C findet eine Darstellung der Theorie der offenbarten Präferenz statt.

${ }^{2}$ Die Implikation folgt aus der Definition der offenbarten Präferenz und der Nutzenmaximierungshypothese.

${ }^{3}$ Die Implikation folgt aus der lokalen Nichtsättigung, welche eine schwächere Annahme als die unterstellte strenge Monotonie der Präferenzordnung ist.
} 
steigender Staatsverschuldung auf der die Gleichgewichtsallokationen beschreibenden Diagonalen nach rechts unten). Die Staatsverschuldung reduziert also die Wohlfahrt der Gesellschaft. ${ }^{1}$ Mit $\tau=\varepsilon \cdot b$ und der notwendigen Bedingung $1-\varepsilon>\tau$ folgt, daß $\forall b \in[0,(1-\varepsilon) / \varepsilon)$ genau ein langfristiges Gleichgewicht existiert, wobei jedes dieser Gleichgewichte mit genau einem Diskontfaktor $\delta$ mit $0<\delta<1$ einhergeht. ${ }^{2} \delta$ ist hierbei eine injektive Funktion von $b$, also $\delta(b){ }^{3}$

Nun sollen die Ergebnisse mit denen verglichen werden, die im DiamondModell resultieren. ${ }^{4}$ Das Diamond-Modell ist ein OLG-Modell mit einer EinSektoren-Technologie, die vermöge einer neoklassischen Produktionsfunktion repräsentiert wird. ${ }^{5}$ Hier entspricht die Ersparnis der Jungen Akteure dem Kapitalstock zu Beginn der folgenden Periode. Die Staatsschuld erhöht den Zinssatz und reduziert zusätzlich die Produktion der Ökonomie, da im Portfolio der Anleger Kapital gegen staatliche Schuldtitel ausgetauscht wird. Die Staatsschuld reduziert aber nicht in jedem Fall die Wohlfahrt. Es sind zwei Fälle zu

${ }^{\prime}$ Die Staatsverschuldung beeinflußt das Nutzenniveau der Akteure über zwei Kanäle. Zum einen bewirkt die Erhöhung des Zinssatzes einen positiven intertemporalen Terms of Trade Effekt, da sich die Verzinsung der Ersparnis erhöht. Zum anderen senkt die Besteuerung das verfügbare Einkommen in der Jugend. Wie die Analyse zeigt, dominiert der zweite Effekt in der Auswirkung auf die Wohlfahrt.

${ }^{2}$ Wie bei der Diskussion der Existenz eines langfristigen Gleichgewichts gezeigt wurde, schneidet die Tauschkurve die Diagonale genau einmal. Ökonomien mit unterschiedlichen Verschuldungsgraden zeichnen sich durch unterschiedliche Tauschkurven aus, welche die Diagonale aber alle genau einmal schneiden müssen. Würde der Staat den Akteuren durch die Besteuerung das gesamte Arbeitseinkommen entziehen $(1-\varepsilon=\tau)$, wäre der Preis der Vermögenstitel $(p=\delta \cdot \varepsilon /(1-\delta))$ null, da dann die Verzinsung der Ersparnis unendlich ist. Dieser Fall kann somit kein ökonomisch sinnvolles Gleichgewicht sein. In einer solchen Situation würden die Vermögenstitel zu einem freien Gut werden, da auf dem Kapitalmarkt ansonsten keine Markträumung möglich wäre. Den Akteuren würde in der Jugend kein Arbeitseinkommen, aus dem sie sparen können, verbleiben. Als Folge könnten sie auf dem Kapitalmarkt keine Vermögenstitel mit positivem Kurswert nachfragen.

${ }^{3}$ Langfristige Gleichgewichte, mit sich unterscheidenden Mengen von sich im Umlauf befindenden Staatsanleihen, gehen mit sich unterscheidenden Diskontfaktoren einher.

${ }^{4}$ Vgl. Diamond (1965), S. $1141 \mathrm{ff}$.

${ }^{5}$ Die Technologie weist konstante Skalenerträge auf, die Produktionsfaktoren Kapital und Arbeit positive aber abnehmende Grenzerträge, und es liegt eine abnehmende Grenzrate der technischen Substitution vor. $\mathrm{Zu}$ den Eigenschaften neoklassischer Produktionsfunktionen siehe z.B. Linde (1981), S. $278 f$. 
unterscheiden. Ist die Ökonomie ohne öffentliche Verschuldung in einem dynamisch effizienten Gleichgewicht (der Zinssatz ist hier mindestens so hoch wie die Wachstumsrate der Bevölkerung), reduziert die Staatsschuld die Wohlfahrt, da der erhöhte Zinssatz mit einem unter den optimalen Wert reduzierten Kapitalstock einhergeht. Ist die Ökonomie ohne öffentliche Verschuldung allerdings in einem dynamisch ineffizienten Gleichgewicht (der Zinssatz ist hier niedriger als die Wachstumsrate der Bevölkerung), erhöht die Staatsschuld die Wohlfahrt, da der erhöhte Zinssatz mit einem in Richtung auf den optimalen Wert reduzierten Kapitalstock einhergeht. ${ }^{1}$ Öffentliche Verschuldung kann sich im Diamond-Modell somit effizienzsteigernd auswirken, was im hier diskutierten Modell nicht möglich ist, da es kein reproduzierbares Produktivkapital gibt.

\subsection{Die Auswirkungen eines staatlichen}

\section{Budgetdefizits}

Sei angenommen, die Ökonomie befindet sich bis Periode 0 in einem langfristigen Gleichgewicht ohne Staatsschuld. In Periode 1 läßt die Regierung der Generation 1 einen nicht erwarteten Transfer $\left(\tau_{1}<0\right)$ zukommen. ${ }^{2}$ Gemäß der staatlichen Budgetrestriktion (4.1) erfordert dieser Transfer eine Emission von

${ }^{1}$ In Diamond (1965) besteuert der Staat, wie im vorliegenden Modell, das Arbeitseinkommen der jungen Akteure. Tin (1997) untersucht den Fall, in dem der Staat auch die Kapitalerträge der alten Akteure besteuert. Befindet sich die Ökonomie in einem dynamisch effizienten Gleichgewicht, so reduziert eine Erhöhung der Staatsverschuldung, bei einem relativ hohen Steuersatz bei der Kapitalertragsteuer, den Zinssatz und erhöht den Kapitalstock. Jetzt ist es möglich, daß die Staatsverschuldung, auch im dynamisch effizienten Bereich, die Wohlfahrt der Akteure langfristig erhöht; vgl. Tin (1997), S. 20. Voraussetzungen hierfür sind ein hoher Steuersatz bei der Kapitalertragsteuer und ein zinsinsensitives Spar- und Investitionsverhalten. ${ }^{2}$ Der Leser mag durch die Tatsache irritiert sein, daß die Akteure von einer Politikmaßnahme überrascht werden können, besitzen sie doch per Annahme vollkommene Voraussicht. Die Eigenschaft vollkommener Voraussicht sollte jedoch als asymptotisches Ergebnis eines Lernprozesses verstanden werden; vgl. Cass und Shell (1980), S. 258f. In einem gegebenen ökonomischen Umfeld besitzen die Akteure weiterhin vollkommene Voraussicht. Der Transfer und die mit ihr einher gehende Staatsverschuldung stellen jedoch einen Strukturbruch im ökonomischen System dar (Ökonomie mit unverschuldetem Staat vs. Ökonomie mit positiver Staatsschuld). Dieser Strukturbruch kann von den Akteuren nicht antizipiert werden. 
$b=-\tau_{1} / p_{1}$ Anleihen. Die Anzahl der Anleihen wird in den folgenden Perioden konstant gehalten. Dies macht die Besteuerung des Arbeitseinkommens der folgenden Generationen in einer Höhe von $\varepsilon \cdot b$ notwendig $\left(\tau_{t}=\varepsilon \cdot b, t=2,3,4 \ldots\right)$. $\mathrm{Da}$ der reguläre Fall betrachtet wird, also der Fall des determinierten langfristigen Gleichgewichts, dauert die Übergangsphase zum neuen langfristigen Gleichgewicht nur eine Periode an. Schon in Periode 2 befindet sich die Ökonomie in ihrem neuen langfristigen Gleichgewicht. ${ }^{1}$

Die Ausgabe der staatlichen Anleihen erhöht die Anzahl der sich im Umlauf befindenden Vermögenstitel von 1 auf $1+b$. Die Gleichgewichtsbedingung für den Kapitalmarkt in Periode 1 lautet somit

$$
s^{\prime}\left(\delta_{2}, 1-\varepsilon-\tau_{1}\right)=\underbrace{p_{1}}_{\begin{array}{c}
\text { Angebot } \\
\text { Aleur } 0
\end{array}}+\underbrace{p_{1} \cdot b}_{\text {Angebor Staat }}=p_{1} \cdot(1+b),
$$

mit $\tau_{1}=-p_{1} \cdot b$ und $\delta_{2}=p_{1} /\left(p_{2}+\varepsilon\right)$. Die Ersparnis des Akteurs 1 muß gleich dem Kurswert der ihm angebotenen Vermögenstitel sein. Die Gütermarkträumung in Periode 2 erfordert

$$
c_{2}^{2}\left(\delta_{3}, 1-\varepsilon-\varepsilon \cdot b\right)+c_{2}^{1}\left(\delta_{2}, 1-\varepsilon-\tau_{1}\right)=1
$$

Der aggregierte Konsum der Akteure muß wie gehabt gleich der Güterproduktion sein (der Staat konsumiert per Annahme keine Güter).

'Die Abweichung vom langfristigen Gleichgewicht dauert nur so lange wie die Störung an. Die Störung ist hier der nicht antizipierte Transfer in Periode 1. Wie in Abschnitt 3.1 gezeigt wurde, besitzt die reguläre Ökonomie genau einen zulässigen Gleichgewichtspfad, dieser ist das langfristige Gleichgewicht. Schon in Periode 2 befindet sich die Ökonomie im neuen langfristigen Gleichgewicht, da alle anderen Pfade nach abzählbar vielen Perioden den durchführbaren Bereich verlassen würden. 


\subsubsection{Die Entwicklung der Zinssätze}

Die Ökonomie befindet sich ab Periode 2 im neuen langfristigen Gleichgewicht. Hierfür ist es erforderlich, daß der Konsum des alten Akteurs 1 gleich dem Konsum der alten Akteure der folgenden Perioden ist, also $c_{2}^{\prime}=c_{t+1}^{t}(t=2,3,4 \ldots)$ gilt. Soll der Konsum des alten Akteurs 1 gleich dem Konsum der alten Akteure der folgenden Periode sein, muß die Verzinsung seiner Ersparnis geringer sein als die Verzinsung der Ersparnis der folgenden Generationen. Dies folgt aus der Tatsache, daß Akteur 1 durch den unerwarteten Transfer, und der mit ihm verbundenen zukünftigen Steuer, über ein höheres Einkommen als die folgenden Generationen verfügt $\left(1-\varepsilon-\tau_{1}>1-\varepsilon-\varepsilon \cdot b\right)$. Ein erhöhtes Einkommen erhöht den Konsum in der Jugend und im Alter (Normalgut-Annahme). Somit muß $\delta_{2}>\delta_{3}$ gelten, denn es gilt $\partial c_{t+1}^{t} / \partial \delta_{t+1}<0$ (unabhängig davon, ob die Güter Bruttosubstitute sind oder nicht). Bei gleicher oder geringerer Verzinsung der Ersparnis wäre es für die folgenden Generationen optimal im Alter weniger zu konsumieren als der alte Akteur 1. Der Effekt des Budgetdefizits ist somit, daß der kurzfristige Zinssatz, im Übergang zum neuen langfristigen Gleichgewicht, niedriger ist als im neuen langfristigen Gleichgewicht, es gilt also $r_{2}<r_{3}$.

Es stellt sich nun die Frage, ob der kurzfristige Zinssatz eine Periode nach Auftreten des staatlichen Budgetdefizits, also in der Übergangsphase zum neuen langfristigen Gleichgewicht, niedriger oder höher als der Zinssatz im alten langfristigen Gleichgewicht ist. Die Veränderung ist nicht eindeutig und hängt vom Verlauf des Einkommensexpansionspfades ab. ${ }^{1}$ Dieser Zusammenhang soll anhand einer Graphik veranschaulicht werden.

Darstellung 4.2 zeigt sowohl das alte bis Periode 0 andauernde und mit $\delta_{0}$ einhergehende langfristige Gleichgewicht der Ökonomie als auch das ab Periode 2 erreichte und mit $\delta_{3}$ einhergehende neue langfristige Gleichgewicht. $b$ wird

\footnotetext{
${ }^{1}$ Vgl. Fried und Howitt (1988), S. 8. Der Einkommensexpansionspfad ist der geometrische Ort aller nutzenmaximierenden Konsumvektoren, bei gegebener Verzinsung und variierendem Einkommen; vgl. z.B. Varian (1992), S. 116f. Die einzige Restriktion, die dem Verlauf des Einkommensexpansionspfades auferlegt ist, folgt aus der Normalgut-Annahme. Aufgrund dieser ist es nicht möglich, daß der Einkommensexpansionspfad einen rückwärts gekrümmten Verlauf aufweist.
} 
durch die Erfordernis $b=-\tau_{1} / p_{1}$ determiniert. Sei $b$ auf diesen Wert fixiert und $p_{1}$ derart gewählt, daß $\delta_{2}=\delta_{0}$ gilt. Dieser Preis werde mit $\tilde{p}_{1}$ bezeichnet $\left(\widetilde{p}_{1}=\delta_{0} \cdot\left(p_{2}+\varepsilon\right)\right)$. Bei $\tilde{p}_{1}$ verläuft die unterbrochen eingezeichnete Budgetgerade der Generation 1 parallel zur Budgetgeraden der Generationen im alten langfristigen Gleichgewicht. Damit die Ökonomie in Periode 2 im neuen langfristigen Gleichgewicht ist, muß $\left(c_{1}^{1}, c_{2}^{1}\right)$ auf der unterbrochen gezeichneten vertikalen Geraden liegen, welche die Abszisse in $(\varepsilon+\varepsilon \cdot b) /\left(1-\delta_{3}\right)$ schneidet. Die Lage des Konsumpunkts von Akteur 1 auf seiner Budgetgeraden wird durch den Verlauf des Einkommensexpansionspfades bestimmt.

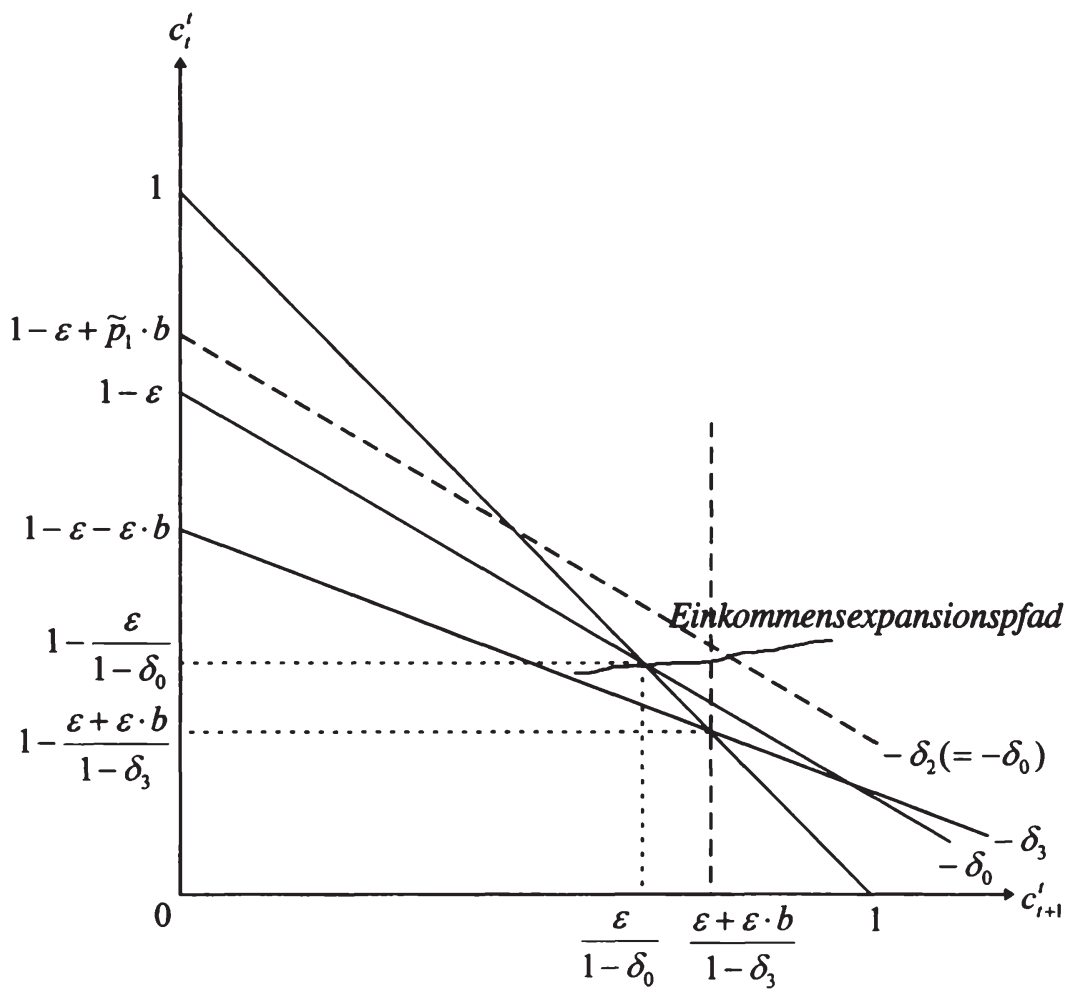

Darst. 4.2: Kurzfristiger Zinseffekt 
Darstellung 4.2 zeigt den Fall, daß dieser Punkt rechts von der gestrichelten vertikalen Geraden liegt. Der alte Akteur 1 konsumiert somit mehr als die alten Akteure der folgenden Generationen. Damit Akteur 1 im Alter weniger konsumiert, ist es notwendig, daß $\delta_{2}$ steigt (wegen $\delta_{2}=\widetilde{p}_{1} /\left(\varepsilon+p_{2}\right)$ ist dies äquivalent dazu, daß $p_{1}$ über $\widetilde{p}_{1}$ steigt, da $p_{2}$ auf seinen neuen langfristigen Wert determiniert ist) bzw. die Verzinsung fällt, da $\partial c_{2}^{1} / \partial \delta_{2}<0$ gilt. Somit ist der kurzfristige Zinssatz eine Periode nach Auftreten des staatlichen Budgetdefizits niedriger als der Zinssatz im alten langfristigen Gleichgewicht, es gilt also $r_{2}<r_{0}<r_{3} \Leftrightarrow \delta_{2}>\delta_{0}>\delta_{3}$. Ganz anders verhält es sich, wenn der Einkommensexpansionspfad derart verläuft, daß der Konsumplan von Akteur 1 bei $\tilde{p}_{1}$ links von der gestrichelten vertikalen Linie liegt. In diesem Fall konsumiert der alte Akteur $1 \mathrm{zu}$ wenig. Jetzt muß $\delta_{2}$ sinken bzw. die Verzinsung steigen. In diesem Fall gilt $r_{0}<r_{2}<r_{3} \Leftrightarrow \delta_{0}>\delta_{2}>\delta_{3}$.

Als nächstes stellt sich die Frage, wie das staatliche Budgetdefizit den langfristigen Zinssatz $r_{t}^{l}$ beeinflußt. Es wird nun gezeigt, daß der langfristige Zinssatz im Übergang zum neuen langfristigen Gleichgewicht monoton steigt, daß also $r_{1}^{\prime}<r_{2}^{l}<r_{3}^{\prime}$ gilt. Dies ist äquivalent dazu, daß der Preis der Vermögenstitel monoton sinkt, also $p_{0}>p_{1}>p_{2}$ gilt (denn es gilt $\left.r_{t+1}^{l}=\varepsilon / p_{t}\right) . p_{0}$ ist der Preis der Vermögenstitel im alten langfristigen Gleichgewicht und $p_{2}$ der im neuen. $\mathrm{Daß} p_{0}>p_{2}$ gilt, wurde schon bei der Analyse der langfristigen Auswirkungen der Staatsschuld gezeigt. $p_{1}>p_{2}$ folgt aus Beziehung (3.1) und $\delta_{2}>\delta_{3}$ (es gilt $\delta_{2}=p_{1} /\left(\varepsilon+p_{2}\right)$ und $\left.\delta_{3}=p_{2} /\left(\varepsilon+p_{2}\right)\right)$.

Eine eindeutige Aussage über die relative Höhe der Vermögenstitelpreise in Periode 0 und Periode 1 läßt sich nur dann treffen, wenn man die Annahme der Determiniertheit des langfristigen Gleichgewichts der Ökonomie verstärkt. Es wird deshalb angenommen, daß der Konsum in der Jugend und der Konsum im Alter Bruttosubstitute sind. Die Steigung der Tauschkurve ist damit über ihren gesamten Verlauf negativ und es gilt $\partial c_{t}^{t} / \partial \delta_{t+1}>0$. Unter dieser Voraussetzung läßt sich durch einen Widerspruchsbeweis leicht zeigen, daß $p_{0}>p_{1}$ gelten muß: Sei angenommen, da $\beta p_{0} \leq p_{1}$ gilt. Dies impliziert, da $\beta c_{1}^{0}\left(=\varepsilon+p_{1}\right)$ $\geq c_{0}^{-1}\left(=\varepsilon+p_{0}\right)$. Gemäß der Gütermarktgleichgewichtsbedingung (3.11) ist dies 
äquivalent dazu, daß $c_{0}^{0}\left(\delta_{0}, 1-\varepsilon\right) \geq c_{1}^{1}\left(\delta_{2}, 1-\varepsilon-\tau_{1}\right) .{ }^{1}$ Das Einkommen des Akteurs 0 ist geringer als das des Akteurs 1 (Akteur 1 erhält einen Transfer). Ein größeres Einkommen wirkt in Richtung eines erhöhten Konsums in der Jugend und im Alter (Normalgut-Annahme). Eine notwendige Bedingung dafür, daß der Jugendkonsum des Akteurs 0 mindestens so hoch ist wie der des Akteurs 1 ist damit, daß der von Akteur 0 erwartete Diskontfaktor höher ist als der von Akteur 1 erwartete (es gilt $\partial c_{t}^{t} / \partial \delta_{t+1}>0$, da die Güter per Annahme Bruttosubstitute sind). $c_{0}^{0} \geq c_{1}^{1}$ impliziert somit $\delta_{0}>\delta_{2}$. Dies stellt jedoch einen Widerspruch zu $p_{0}>p_{2}$ und der Annahme $p_{0} \leq p_{1}$ dar, denn $\delta_{2}=p_{1} /\left(p_{2}+\varepsilon\right)$ $\geq p_{0} /\left(p_{2}+\varepsilon\right)>p_{0} /\left(p_{0}+\varepsilon\right)=\delta_{0}{ }^{2}$. Somit wurde gezeigt, daß die Halter der Vermögenstitel, auf dem Weg zum neuen langfristigen Gleichgewicht, einen Kapitalverlust hinnehmen müssen. ${ }^{3}$

Mit der Erkenntnis, daß $p_{-1}=p_{0}>p_{1}>p_{2}=p_{3}\left(\Leftrightarrow r_{0}^{l}=r_{1}^{l}<r_{2}^{\prime}<r_{3}^{l}=r_{4}^{l}\right)$ gilt und der Arbitragebedingung (3.2), erhält man eine vollständige Beschreibung der durch das Budgetdefizit induzierten Zinsdynamik. Hierbei gilt:

${ }^{1}$ Akteur 0 erwartet einen Diskontfaktor von $\delta_{0}$, da sich die Ökonomie in Periode 0 noch im langfristigen Gleichgewicht ohne Staatsschuld befindet. Tatsächlich realisiert sich aufgrund des nicht antizipierten Budgetdefizits in Periode 1 ein Diskontfaktor $\delta_{1}$, der höher als $\delta_{0}$ ist (dies wird weiter unten gezeigt). Somit unterläuft Generation 0 ein Erwartungsfehler. Sie antizipiert nicht das in Periode 1 auftretende staatliche Budgetdefizit. Geht man wie hier davon aus, da $B$ ein staatliches Budgetdefizit ein neues noch nie aufgetretenes Ereignis ist, dann bedeutet das Auftreten eines durch das Budgetdefizit erzeugten Erwartungsfehlers nicht unbedingt eine Verletzung der Annahme der vollkommenen Voraussicht. Das Budgetdefizit stellt einen Strukturbruch in der Dynamik der Ökonomie dar (der Übergang von der Ökonomie ohne Staatsschuld hin zu einer Ökonomie mit positiver Staatsschuld). Innerhalb eines bestehenden ökonomischen Umfelds besitzen die Akteure weiterhin vollkommene Voraussicht, sie können nur durch ein seltenes Ereignis eines Strukturbruchs überrascht werden.

${ }^{2}$ Hätte man die Annahme der Determiniertheit des langfristigen Gleichgewichts nicht verstärkt, wäre die Implikation, die zu $\delta_{0}>\delta_{2}$ führt, nicht möglich, und der Widerspruchsbeweis würde nicht funktionieren. Determiniertheit läßt auch Bruttokomplemente zu. Bei Bruttokomplementen impliziert die Tatsache, daß der Jugendkonsum von Akteur 0 mindestens so hoch ist wie der von Akteur 1, daß $\delta_{0}<\delta_{2}$ gilt.

${ }^{3}$ Wie aus der Diskussion der Arbitragebedingung (3.2) folgt, ist eine Erhöhung des langfristigen Zinssatzes ein Indikator für den Kapitalverlust, der sich vollständig auf den Akteur auswirkt, welcher zum Zeitpunkt der Zinsveränderung die Vermögenstitel hält. 


$$
\begin{aligned}
& r_{0}=\frac{\varepsilon}{p_{-1}}+\underbrace{\frac{p_{0}-p_{-1}}{p_{-1}}}_{=0}=r_{0}^{l}-\underbrace{\frac{r_{1}^{l}-r_{0}^{l}}{r_{1}^{l}}}_{=0} \Leftrightarrow r_{0}=r_{0}^{l}, \\
& r_{1}=\frac{\varepsilon}{p_{0}}+\underbrace{\frac{p_{1}-p_{0}}{p_{0}}}_{<0}=r_{1}^{l}-\underbrace{\frac{r_{2}^{l}-r_{1}^{l}}{r_{2}^{l}}}_{>0} \Leftrightarrow r_{1}<r_{1}^{\prime}, \\
& r_{2}=\frac{\varepsilon}{p_{1}}+\underbrace{\frac{p_{2}-p_{1}}{p_{1}}}_{<0}=r_{2}^{l}-\underbrace{\frac{r_{3}^{l}-r_{2}^{l}}{r_{3}^{l}}}_{>0} \Leftrightarrow r_{2}<r_{2}^{l}, \\
& r_{3}=\frac{\varepsilon}{p_{2}}+\underbrace{\frac{p_{3}-p_{2}}{p_{2}}}_{=0}=r_{3}^{l}-\underbrace{\frac{r_{4}^{l}-r_{3}^{l}}{r_{4}^{l}}}_{=0} \Leftrightarrow r_{3}=r_{3}^{l} .
\end{aligned}
$$

Bis Periode 0 sind der kurzfristige und der langfristige Zinssatz auf ihrem alten langfristigen Niveau, wobei die Zinsstruktur flach ist. In Periode 1 induziert die nicht antizipierte Emission der Staatsanleihen ein Absinken der Vermögenstitelpreise. Dies erzeugt ein Kapitalverlust, den der Akteur 0 vollständig tragen muß (der von ihm gehaltene Anteilschein verliert an Wert). Somit sinkt der kurzfristige Zinssatz zwischen Periode 0 und 1 auf $r_{1}\left(<r_{0}\right)$, wobei die Zinsstruktur in Periode 1 normal ist.

Wie die Diskussion der Darstellung 4.2 gezeigt hat, kann das Modell keine eindeutige Aussage dazu machen, ob der kurzfristige Zinssatz in Periode 2 geringer oder höher ist als der kurzfristige Zinssatz im alten langfristigen Gleichgewicht. Es sind also zwei Fälle zu unterscheiden. ${ }^{1}$ Der erste Fall, der auch in Darstellung 4.2 gezeigt wird ist der, daß $r_{2}<r_{0}$ gilt. In diesem Fall ist aber nicht klar, wie aus den Gleichungen (4.10) und (4.11) ersichtlich wird, ob der kurzfristige Zinssatz zwischen Periode 1 und Periode 2 sinkt oder steigt. Zwar ist die

\footnotetext{
${ }^{1}$ Wie man aus den Gleichungen (4.9) und (4.11) erkennt, unterscheiden sich die beiden Fälle dadurch, daß im einen Fall die positive Differenz in der Ertragskomponente der Renditen der Periode 2 und der Periode 0 durch den Kapitalverlust in der Periode 2 überkompensiert wird und im anderen Fall nicht.
} 
Ertragskomponente der Rendite in Periode 2 größer als in Periode $1\left(p_{1}<p_{0}\right.$ $\left.\Leftrightarrow \varepsilon / p_{1}>\varepsilon / p_{0}\right)$, das Modell macht aber keine Aussagen über die Ausprägung der Kapitalverlustkomponente in den beiden Perioden. Der zweite Fall ist der, daß $r_{2}>r_{0}$ gilt. Hier läßt sich eine eindeutige Aussage treffen, wie sich der kurzfristige Zinssatz zwischen Periode 1 und Periode 2 entwickelt. Da $r_{1}<r_{0}$ gilt, muß der kurzfristige Zinssatz zwischen Periode 1 und Periode 2 steigen. Egal welcher Fall vorliegt, muß die Zinsstruktur in Periode 2 normal sein. Dies ist auch zu erwarten gewesen, da die Vermögenstitel zwischen Periode 1 und Periode 2 an Wert verlieren.

In Periode 3 stimmen der kurzfristige und der langfristige Zinssatz wieder überein. Der Wert der Vermögenstitel bleibt ab Periode 2 konstant, da sich die Ökonomie ab dieser Periode im neuen langfristigen Gleichgewicht befindet. Der kurzfristige und der langfristige Zinssatz steigen zwischen Periode 2 und Periode 3, wobei die Veränderung des kurzfristigen Zinssatzes ausgeprägter ist als die des langfristigen Zinssatzes (die Zinsstruktur war in Periode 2 noch normal, in Periode 3 ist sie flach). Zum einen erhöht sich der kurzfristige Zinssatz in Periode 3 durch seine vergrößerte Ertragskomponente $\left(p_{2}<p_{1} \Leftrightarrow \varepsilon / p_{2}>\varepsilon / p_{1}\right)$, zum anderen findet in Periode 3 kein Kapitalverlust statt. Darstellung 4.3 veranschaulicht die möglichen Zinspfade.

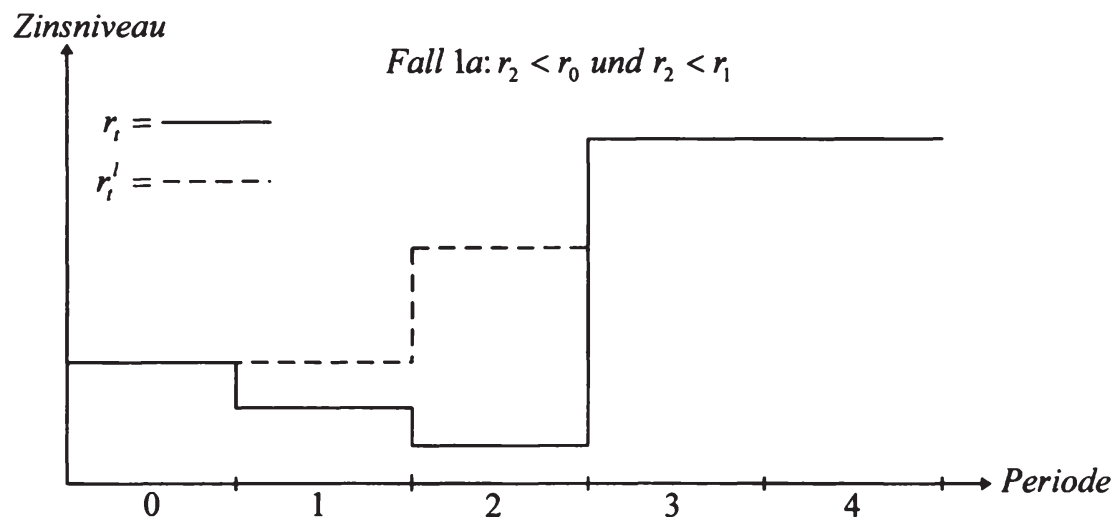



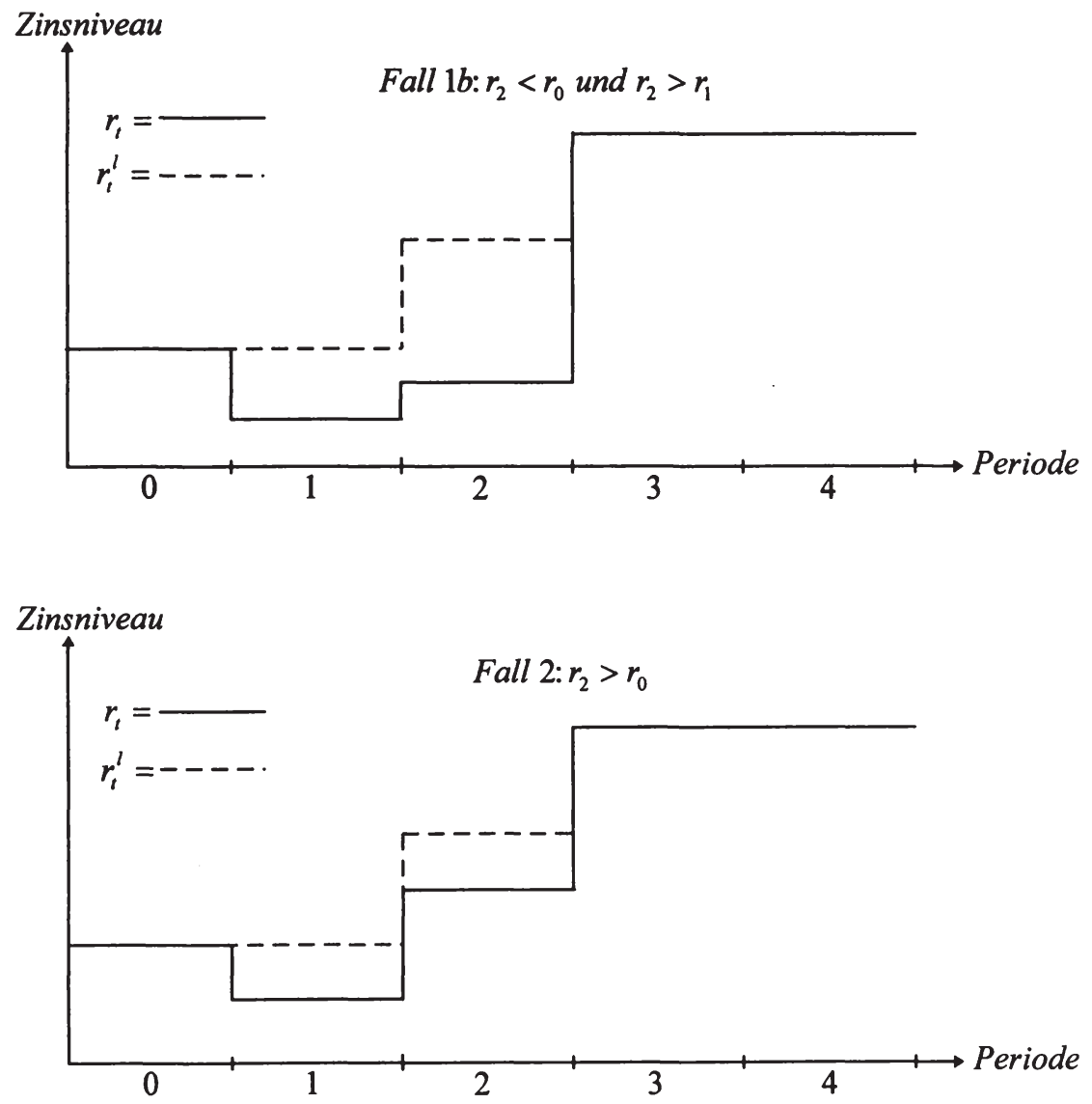

Darst. 4.3: Mögliche Zinsdynamik

\subsubsection{Die Auswirkung auf die Wohlfahrt}

Wie wirkt sich nun das staatliche Budgetdefizit auf die Wohlfahrt der betroffenen Generationen aus? Akteur 0 wird in Periode 1 von dem unerwarteten staatlichen Budgetdefizit überrascht. $c_{0}^{0}$ bleibt vom Budgetdefizit unberührt, da es nicht antizipiert wird. Der Akteur muß im Alter allerdings ein Kapitalverlust 
hinnehmen, so daß sein Konsum im Vergleich zur Situation ohne Budgetdefizit geringer ist $\left(\varepsilon+p_{1}<\varepsilon+p_{0}\right)$. Die Wohlfahrt von Akteur 0 wird somit verringert. Das Einkommen von Akteur 1 Erhöht sich durch den ihm zukommenden staatlichen Transfer. Sein Konsum im Alter erhöht sich ( $c_{2}^{1}$ steigt auf den neuen stationären Wert). Wegen $p_{0}>p_{1}$, steigt auch $c_{1}^{1}$ im Vergleich zur Situation ohne Budgetdefizit, denn wegen $p_{0}>p_{1}$ gilt $c_{1}^{0}\left(=\varepsilon+p_{1}\right)<c_{0}^{-1}\left(=\varepsilon+p_{0}\right)$. Dies ist gemäß der Gütermarktgleichgewichtsbedingung (3.11) äquivalent $\mathrm{zu} c_{0}^{0}$ $<c_{1}^{1}$. Somit steigt die Wohlfahrt von Akteur 1. Die Wohlfahrt der Akteure der folgenden Generationen wird, wie bei der Analyse der Auswirkungen der Staatsschuld gezeigt wurde, reduziert.

Es könnte nun der Wunsch entstehen einen Wohlfahrtsvergleich in dem Sinne durchzuführen, daß man die erreichbare gesellschaftliche Wohlfahrt, in einer Ökonomie mit positiver Staatsverschuldung, der gesellschaftlichen Wohlfahrt, in einer Ökonomie ohne Staatsverschuldung, gegenüberstellt. Ein solcher Vergleich ist jedoch aus folgenden Gründen wenig sinnvoll:

- Der von den Akteuren erreichbare Nutzen hängt zum einen von ihrem verfügbaren Einkommen ab und zum anderen von der Verzinsung ihrer Ersparnis. Wie die Diskussion der Zinsdynamik zeigt, ist diese indeterminiert. Damit ist es methodisch nicht möglich, den Generationen 0 und 1 eine bestimmte Verzinsung ihrer Ersparnis zuzuordnen.

- Ein sinnvoller Ansatz zur Bestimmung einer sozialen Wohlfahrtsfunktion wäre ein utilitaristisches Vorgehen. ${ }^{1}$ Da ein intertemporales Modell vorliegt, ist es jedoch notwendig, den Nutzen der einzelnen Generationen zeitlich zu homogenisieren. Für die Höhe der für dieses Vorhaben notwendigen sozialen Diskontierungsrate gibt es jedoch a priori keine theoretisch gehaltvollen Determinanten. Es ist einfach nicht klar, welche Diskontierungsrate adäquat ist. Eine durch den Zinssatz determinierte Diskontierungsrate erscheint plausibel,

\footnotetext{
${ }^{1}$ Eine utilitaristische soziale Wohlfahrtsfunktion zeichnet sich dadurch aus, daß in ihr die individuellen Nutzen zu einem Kollektivnutzen aggregiert werden. Ein alternativer Ansatz ist der von Rawls (1971). Hier gleicht der gesamtgesellschaftliche Nutzen dem des Akteurs mit dem geringsten Nutzenniveau. Für einen Wohlfahrtsvergleich, wie er hier angestrebt wird, ist letzterer Ansatz offensichtlich unbrauchbar.
} 
dieser wird aber durch den Verschuldungsgrad des Staates verändert. Eine vom Zinssatz unabhängige konstante Diskontierungsrate scheint auch plausibel, ihre Größe wäre jedoch arbiträr. ${ }^{1}$ Beide Ansätze sind wenig zufriedenstellend.

Die beiden aufgeführten prinzipiellen Probleme machen eine grundlegende Wohlfahrtsanalyse methodisch unmöglich. Die einzige Möglichkeit ist der oben durchgeführte qualitative Wohlfahrtsvergleich, bei dem analysiert wird, welche Generationen besser und welche schlechter gestellt werden.

Die Ergebnisse lassen sich wieder mit denen im Diamond-Modell vergleichen. ${ }^{2}$ Hier bleiben die Akteure, die in der Periode eines Budgetdefizits alt sind, von diesem unberührt. Dies resultiert aus der Tatsache, daß es innerhalb des Diamond-Modells keine Kapitalverluste bzw. Kapitalgewinne gibt. Die Akteure sparen in Form von Produktivkapital oder staatlichen Schuldtiteln, die jedoch nur eine Laufzeit von einer Periode besitzen. Die Rendite aus dem Kapitalstock wird durch das physische Grenzprodukt des Produktivkapitals bestimmt, und da Kapital und Schuldtitel vollkommene Substitute im Portfolio der Anleger sind, auch die Verzinsung der Schuldtitel. Die in der Periode eines Budgetdefizits jungen Akteure profitieren auch im Diamond-Modell von diesem. Zum einen bleibt ihr Arbeitslohn vom Budgetdefizit unberührt, da er vom existierenden Kapitalstock determiniert wird, und zum anderen erhöht sich die Verzinsung ihrer Ersparnis im Verhältnis zur Verzinsung im alten langfristigen Gleichgewicht, da der Zinssatz auf dem Pfad hin zum neuen langfristigen Gleichgewicht monoton steigt. Das im vorliegenden Modell auftretende Phänomen, daß der kurzfristige Zinssatz vorübergehend unter den Zinssatz im alten langfristigen Gleichgewicht fällt, ist im Diamond-Modell nicht möglich. Dies ist auch plausibel, da das Absinken des kurzfristigen Zinssatzes durch einen, im DiamondModell nicht möglichen, Kapitalverlust erzeugt wird.

\footnotetext{
1 Beispiele für einen utilitaristischen Ansatz, mit einer exogen vorgegebenen sozialen Diskontierungsrate, bieten Calvo und Obstfeld (1988) und Buiter und Kletzer (1991).

${ }^{2}$ Siehe dazu Persson (1985), S. 73f.
} 


\section{Ein Zwei-Länder-OLG-Modell der Weltwirtschaft}

Sei nun vorerst wieder ein passiver Staat unterstellt (keine Transfers oder Steuern) aber dafür die Existenz eines weiteren Landes angenommen. Spezifische Charakteristika des zweiten Landes werden in Großbuchstaben geschrieben. Die bisher betrachtete Ökonomie wird Inland genannt, die zweite Ökonomie Ausland. Das Inland und das Ausland sind bezüglich der demographischen Struktur, der Ausstattung mit Arbeitskraft und der verfügbaren Technologie identisch. Auch die intertemporale Nutzenfunktion der ausländischen Akteure ist streng quasikonkav, differenzierbar und streng ansteigend, es gilt aber $U \neq u$. Die Akteure der beiden Länder unterscheiden sich also nur durch ihre intertemporale Nutzenfunktion. Sei zu Beginn die Dynamik bei Autarkie betrachtet, also die Situation, in der zwischen den Ländern keine wirtschaftlichen Beziehungen bestehen. Das intertemporale Nutzenmaximierungsproblem der ausländischen Akteure lautet analog zu (3.1):

$$
U\left(C_{t}^{t}, C_{t+1}^{t}\right) \rightarrow \underset{C_{t}^{t}, C_{t+1}^{t} !}{\operatorname{Max} !}
$$

unter der $N B$

$$
\begin{aligned}
C_{t}^{t}+S^{t} & =1-\varepsilon & & \text { (Budgetrestriktion Jugend) } \\
C_{t+1}^{t} & =\Delta_{t+1}^{-1} \cdot S^{t} & & \text { (Budgetrestriktion Alter) }
\end{aligned}
$$

Aus den Bedingungen erster Ordnung der Optimierung folgt analog zu (3.9):

$$
\frac{\frac{\partial U\left(C_{t}^{t}, C_{t+1}^{t}\right)}{\partial C_{t+1}^{t}}}{\frac{\partial U\left(C_{t}^{t}, C_{t+1}^{t}\right)}{\partial C_{t}^{t}}}=\Delta_{t+1} \stackrel{\text { Budgetres. }}{\longrightarrow} \text { optimaler Konsumvektor }\left(C_{t}^{t}, C_{t+1}^{t}\right),
$$

mit

$$
P_{t}=\Delta_{t+1} \cdot\left(\varepsilon+P_{t+1}\right), \text { wobei } \Delta_{t+1}:=\frac{1}{1+R_{t+1}}
$$


Man bemerke, daß der mit dem ausländischen Anteilschein verbundene Dividendenstrom mit dem inländischen übereinstimmt. Auch im Ausland wird in jeder Periode eine Gütereinheit produziert. Der Gewinn aus dieser Produktion beträgt auch im Ausland in jeder Periode $\varepsilon$ und damit das Arbeitseinkommen $1-\varepsilon$. Die Gleichheit des Dividenden- und Einkommensstroms resultiert aus der Gleichheit der Produktionssektoren in den beiden Ländern. Die in jeder Periode in den beiden Ländern produzierten Güter sind identisch. Somit wird bei der Untersuchung der Auswirkungen einer Öffnung der beiden Ökonomien auf die intertemporalen Aspekte des Außenhandels abgestellt. ${ }^{1}$

Aus der Optimierung folgen die Konsumfunktionen der jungen und der alten ausländischen Akteure, sowie ihre Sparfunktion: $C_{t}^{t}=C_{t}^{t}\left(\Delta_{t+1}, 1-\varepsilon\right), C_{t+1}^{t}$ $=C_{t+1}^{t}\left(\Delta_{t+1}, 1-\varepsilon\right)$ und $S^{t}\left(\Delta_{t+1}, 1-\varepsilon\right)$. Es wird angenommen, daß auch der Gleichgewichtspfad des Auslands wie der des Inlands determiniert ist. ${ }^{2}$ Somit besitzt auch das Ausland genau einen Gleichgewichtspfad. Es muß sich von Anfang an in seinem langfristigen Gleichgewicht befinden. Unter Verwendung von Beziehung (5.3) erhält man $\Delta=P /(P+\varepsilon) \Leftrightarrow P=\Delta \cdot \varepsilon /(1-\Delta)$ Der Konsum der jungen Ausländer im langfristigen Gleichgewicht beträgt $1-\varepsilon-P$ $=1-\varepsilon /(1-\Delta)$ und der Konsum der alten Ausländer $\varepsilon+P=\varepsilon /(1-\Delta)$. Das langfristige ausländische Autarkiegleichgewicht geht somit mit dem stationären Konsumvektor $(1-\varepsilon /(1-\Delta), \varepsilon /(1-\Delta))$ einher. Eine notwendige Bedingung

${ }^{1}$ Es interessieren also weiterhin nur die intertemporalen Relativpreise (die Zinssätze) und nicht die intratemporalen Relativpreise. Intratemporale Relativpreise wären zum einen der reale Wechselkurs (das Verhältnis der nationalen Preisniveaus) und die intratemporalen Terms of Trade (der Relativpreis von Importen ausgedrückt in Exporten). Beide intratemporalen Relativpreise sind im vorliegenden Modell irrelevant (es existiert in der Welt nur ein einziges Gut pro Periode, das frei gehandelt wird). Eine Veränderung des Zinssatzes ruft eine Veränderung der intertemporalen Terms of Trade hervor. Der Diskontfaktor ist der Gegenwartspreis des Konsums der nächsten Periode und der Kehrwert des Diskontfaktors (also die Verzinsung der Ersparnis) der Preis des gegenwärtigen Konsums, ausgedrückt in zukünftigen Gütereinheiten. Eine Erhöhung des Zinssatzes verbessert die intertemporalen Terms of Trade des Landes, dessen Güterexport in der Gegenwart positiv ist. Zur Unterscheidung von intratemporalen und intertemporalen Relativpreisen siehe Obstfeld und Rogoff (1996), S. 25 und S. 235ff, oder auch Frenkel und Razin (1996), S. 269ff. Persson und Svensson (1985), Matsuyama (1988) und Serra (1991) bieten Analysen, die auf die Auswirkungen von Außenhandel auf intratemporale Relativpreise abstellen.

${ }^{2}$ Die Steigung der ausländischen Tauschkurve muß im langfristigen Gleichgewicht somit kleiner als eins sein. 
dafür, daß sich die Pfade der beiden Länder (bei identischen Produktionssektoren und demographischen Strukturen) unterscheiden ist, daß $u \neq U$ gilt.

\subsection{Die integrierte Weltwirtschaft}

Nun wird der Fall betrachtet, daß sich die beiden Länder dem Außenhandel öffnen. Es ist somit ein freier Handel von Gütern und Vermögenstiteln möglich (Laissez-Faire-Regime). Die integrierte Weltwirtschaft zeichnet sich durch die Gültigkeit des Gesetzes des einen Preises aus. Bei der Abwesenheit von Transportkosten und Handelsbeschränkungen, und bei der Existenz einer kompetitiven Marktstruktur, müssen gleiche Güter überall einen gleichen Preis besitzen. Wäre dies nicht der Fall, wären Arbitragegeschäfte möglich. ${ }^{1}$ Die Anteilscheine an den nationalen Produktionstechniken sind in ihrer Funktion als Anlageinstrument vollkommene Substitute, da sich die Gewinne international nicht unterscheiden. Im Gleichgewicht gilt also:

$$
p_{t}=P_{t}=: \bar{p}_{t}, \forall t \text { und } \delta_{t+1}=\Delta_{t+1}=: \bar{\delta}_{t+1}, \forall t \text {. }
$$

Und damit

$$
\bar{p}_{t}=\bar{\delta}_{t+1} \cdot\left(\varepsilon+\bar{p}_{t+1}\right), \quad \text { wobei } \bar{\delta}_{t+1}=\frac{1}{1+\bar{r}_{t+1}}
$$

Werte, die in der integrierten Weltwirtschaft determiniert werden, werden mit einem Querstrich gekennzeichnet. Der Preis der Vermögenstitel und der Diskontfaktor sind im Inland und im Ausland in jeder Periode gleich (der inländische und der ausländische Anteilschein sind in ihrer Funktion als Anlagemedium vollkommene Substitute, da beide mit einem unendlichen Dividendenstrom

\footnotetext{
' Vgl. Obstfeld und Rogoff (1996), S. 202. Bei sich international unterscheidenden Preisen erwerben Arbitrageure die Güter im Land mit dem niedrigeren Preis und veräußern sie im Land mit dem höheren Preis. Die Arbitragegeschäfte führen zu sich international angleichenden Preisen. Der Gleichgewichtspreis muß somit international übereinstimmen.
} 
von $\varepsilon$ Gütereinheiten pro Periode verbunden sind). Sie werden auf dem Weltgütermarkt und dem Weltkapitalmarkt determiniert.

Die Weltkonsumnachfrage der Jungen der Periode $t$ beträgt

$$
\bar{c}_{t}^{\prime}\left(\bar{\delta}_{t+1}, 1-\varepsilon, 1-\varepsilon\right):=c_{t}^{t}\left(\bar{\delta}_{t+1}, 1-\varepsilon\right)+C_{t}^{t}\left(\bar{\delta}_{t+1}, 1-\varepsilon\right)
$$

und die der Alten dieser Periode

$$
\bar{c}_{t}^{t-1}\left(\bar{\delta}_{t}, 1-\varepsilon, 1-\varepsilon\right):=c_{t}^{t-1}\left(\bar{\delta}_{t}, 1-\varepsilon\right)+C_{t}^{t-1}\left(\bar{\delta}_{t}, 1-\varepsilon\right) .
$$

Die Weltersparnis der Generation $t$ beträgt

$$
\bar{s}^{t}\left(\bar{\delta}_{t+1}, 1-\varepsilon, 1-\varepsilon\right):=s^{t}\left(\bar{\delta}_{t+1}, 1-\varepsilon\right)+S^{t}\left(\bar{\delta}_{t+1}, 1-\varepsilon\right) .
$$

Die Weltgütermarkträumung erfordert:

$$
\bar{c}_{t}^{t}\left(\bar{\delta}_{t+1}, 1-\varepsilon, 1-\varepsilon\right)+\hat{c}_{t}^{t-1}\left(\bar{\delta}_{t}, 1-\varepsilon, 1-\varepsilon\right)=2, \forall t .
$$

Die Weltgüterproduktion beträgt zwei Gütereinheiten pro Periode. Damit der Weltgütermarkt geräumt wird, muß die Summe aus aggregiertem Konsum der Jungen und aggregiertem Konsum der Alten zwei betragen. Die Weltkapitalmarkträumung erfordert:

$$
\bar{s}^{\prime}\left(\bar{\delta}_{t+1}, 1-\varepsilon, 1-\varepsilon\right)=\bar{p}_{t} \cdot 2, \forall t .
$$

Die Ersparnis der jungen Akteure (die Differenz zwischen aggregiertem Arbeitseinkommen und aggregiertem Konsum der Jungen) muß gleich dem Kurswert der von den alten Akteuren angebotenen Vermögenstitel sein (letztere bieten die beiden Anteilscheine an den nationalen Produktionstechniken an).

Ein Walras-Gleichgewicht der integrierten Weltwirtschaft läßt sich wie folgt definieren: 
Eine Folge von Vermögenstitelpreisen $\left\{\bar{p}_{t}\right\}_{t \in Z}$ und die mit ihr einhergehende Folge von nationalen Konsumplänen $\left\{\left(c_{t}^{t}, c_{t+1}^{t}\right),\left(C_{t}^{t}, C_{t+1}^{t}\right)\right\}_{t \in \mathcal{Z}}$ beschreiben ein Walras-Gleichgewicht der integrierten Weltwirtschaft bei vollkommener Voraussicht, wenn die Konsumpläne die Optimalitätsbedingung (3.9) bzw. (5.2) erfüllen und der Weltgütermarkt sowie der Weltkapitalmarkt in jeder Periode geräumt werden, also die Gleichungen (5.9) und (5.10) erfüllt sind.

Bei der Analyse der Gleichgewichtsdynamik der integrierten Weltwirtschaft reicht es wieder aus, sich auf einen der beiden Märkte zu konzentrieren (Walras-Gesetz).

In Darstellung 5.1 wird die Existenz eines Gleichgewichtspfades der integrierten Weltwirtschaft hergeleitet. Es sind sowohl die Gleichgewichte der beiden Länder bei Autarkie als auch das Gleichgewicht der integrierten Weltwirtschaft eingezeichnet. Betrachtet wird der Fall, daß die Akteure des Auslands eine höhere Zeitpräferenz besitzen als die Akteure des Inlands (die Inländer sind geduldiger als die Ausländer). Dies spiegelt sich in einer ausländischen Tauschkurve wieder, die oberhalb der Tauschkurve des Inlands verläuft. ${ }^{1}$ Die Annahme bezüglich der Zeitpräferenz im Inland und im Ausland wird im weiteren Verlauf der Arbeit aufrecht gehalten. Der Diskontfaktor im langfristigen Autarkiegleichgewicht des Inlands ist höher als der Diskontfaktor im langfristigen Autarkiegleichgewicht des Auslands, es gilt also $\delta>\Delta$. Dies ist gemäß Beziehung (3.1) bzw. (5.3) äquivalent dazu, daß der Zinssatz im langfristigen Gleichgewicht des Auslands höher ist als im langfristigen Gleichgewicht des Inlands $(r<R)$. Dies ist ökonomisch plausibel, da den Ausländern aufgrund ihrer größeren Ungeduld ein größerer Anreiz zum Sparen geboten werden muß. Dieser größere Anreiz ist der höhere Zinssatz.

Die Welttauschkurve erhält man durch Aggregation der nationalen Tauschkurven. Sie läßt sich als geometrischer Ort aller nutzenmaximierenden Weltkonsumpläne interpretieren. Unter Weltkonsumplan wird hier die Aufteilung des aggregierten Konsums, in aggregierten Jugendkonsum und aggregierten Alters-

${ }^{1}$ Bei jeder Verzinsung der Ersparnis ist das Verhältnis von optimaler Konsummenge in der Jugend und optimaler Konsummenge im Alter im Inland niedriger als im Ausland. Zur Rolle der Zeitpräferenz in dynamischen Modellen, siehe z.B. Epstein und Hynes (1983). 
konsum, verstanden. Der Aggregationsprozeß wird in Darstellung 5.1 für die beiden Diskontfaktoren $\delta$ und $\Delta$ exemplifiziert. Sehen sich die Akteure einem Diskontfaktor von $\bar{\delta}_{t+1}=\delta$ gegenüber, so lautet der optimale Konsumplan des inländischen Akteurs $(f, c)$ und der des ausländischen Akteurs $(h, a)$. Der Weltkonsumplan lautet dann $A(=(f+h, c+a))$. Entsprechend erhält man den Weltkonsumplan bei $\bar{\delta}_{t+1}=\Delta$. Dieser wird durch $B(=(e+g, d+b))$ gegeben. Die Aggregationsprozedur wird auch aus den Gleichungen (5.6) und (5.7) offensichtlich.

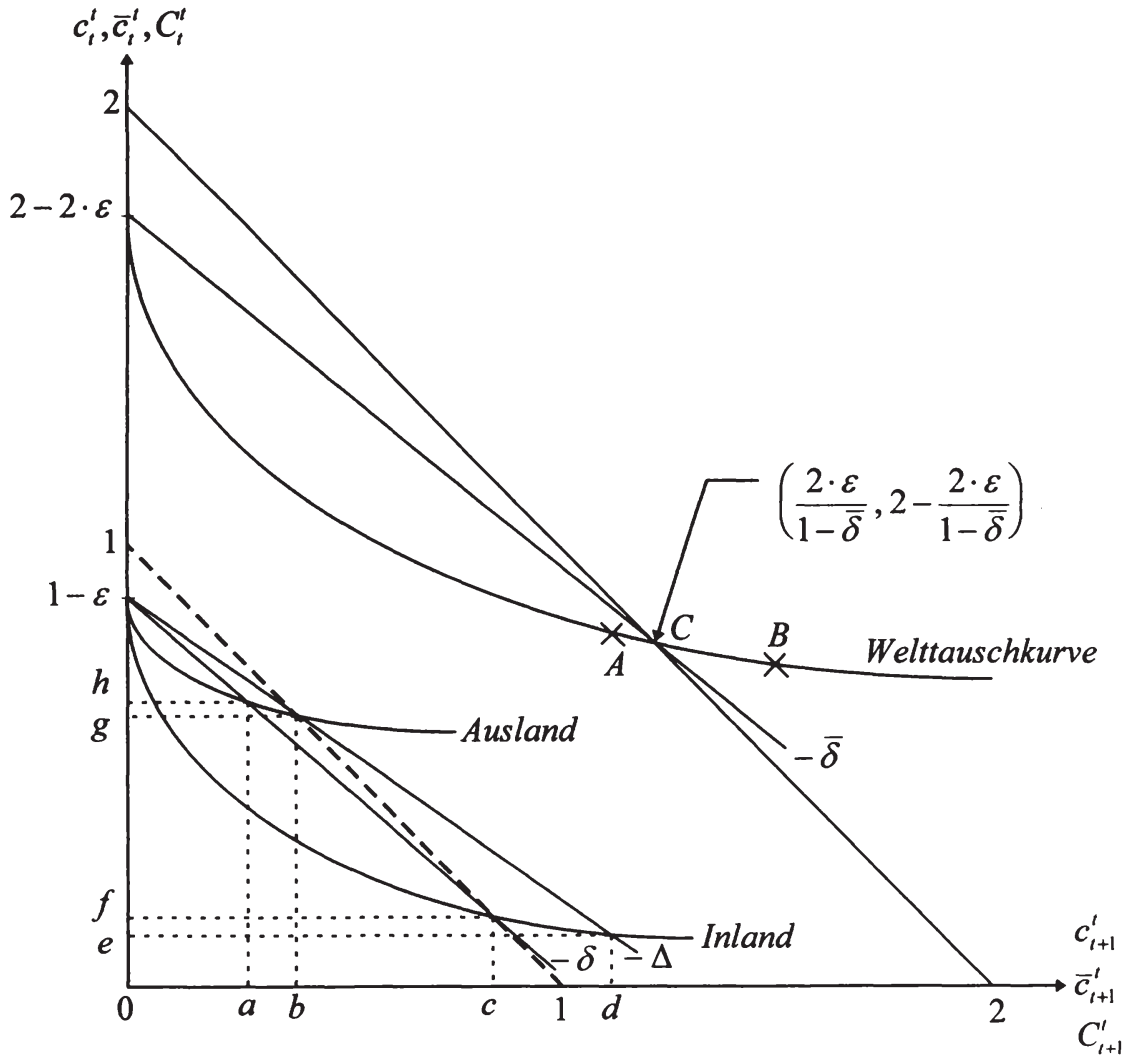

Darst. 5.1: Die integrierte Weltwirtschaft 
Die Dynamik der Weltwirtschaft läßt sich nun unter Zuhilfenahme der Welttauschkurve analysieren. Es wurde angenommen, daß der Gleichgewichtspfad der beiden Länder bei Autarkie determiniert ist. Diese Annahme ist hinreichend dafür, daß auch der Gleichgewichtspfad der Weltwirtschaft determiniert ist. ${ }^{1}$ Die Dynamik der integrierten Weltwirtschaft läßt sich analog zum Fall der geschlossenen Ökonomie herleiten.

Darstellung 5.2 veranschaulicht die Gleichgewichtsdynamik auf dem Weltgütermarkt. Auf der Ordinate ist der Weltjugendkonsum abgetragen und auf der Abszisse der Weltalterskonsum. Ein temporäres Weltgütermarktgleichgewicht zeichnet sich dadurch aus, daß die Summe aus Weltjugendkonsum und Weltalterskonsum zwei ist. Somit stellen alle Weltallokationen, die auf der eingezeichneten Diagonalen liegen, Weltgütermarktgleichgewichte dar.

Sei $\bar{\delta}_{t+1}$ der Diskontfaktor zwischen den Perioden $t$ und $t+1$, dann wird der Weltkonsumplan der Generation $t$ durch Punkt $A$ gegeben. Die Gütermarkträumung in Periode $t+1$ erfordert, daß der Weltkonsumplan der Generation $t+1$ in Punkt $B$ liegt. Hierfür muß der Diskontfaktor zwischen den Perioden $t+1$ und $t+2$ derart sein, da $\beta$ das Aggregat aus dem optimalen Konsumplan des inländischen Akteurs $t+1$ und dem optimalen Konsumplan des ausländischen Akteurs $t+1 \quad B$ ergibt. Der in der Darstellung eingezeichnete Diskontfaktor $\bar{\delta}_{t+2}$ erfüllt diese Bedingung. Man erkennt, daß der Diskontfaktor im Zeitablauf steigt bzw. die Verzinsung fällt. Die Anteilscheine verlieren an Wert. Die Halter der Scheine müssen somit einen Kapitalverlust hinnehmen.

${ }^{1}$ Diese Suffizienz wird aus der Aggregationsprozedur ersichtlich, wenn man extrem nicht glatte nationale Tauschkurven ausschließt. Betrachtet man den Fall, daß die nationalen langfristigen Gleichgewichte sich unterscheiden (wie das in Darstellung 5.1 der Fall ist) so gilt $\delta \neq \bar{\delta} \neq \Delta$. Es ist nun prinzipiell denkbar, daß trotz Determiniertheit der nationalen langfristigen Gleichgewichte mindestens eine der nationalen Tauschkurven im Bereich von $\bar{\delta}$ eine Steigung von größer eins aufweist, so daß unter Umständen das langfristige Gleichgewicht der Weltwirtschaft indeterminiert ist (die Welttauschkurve weist dann im langfristigen Gleichgewicht eine Steigung von größer eins auf). Derartige Extremfälle werden im Folgenden ausgeschlossen. 


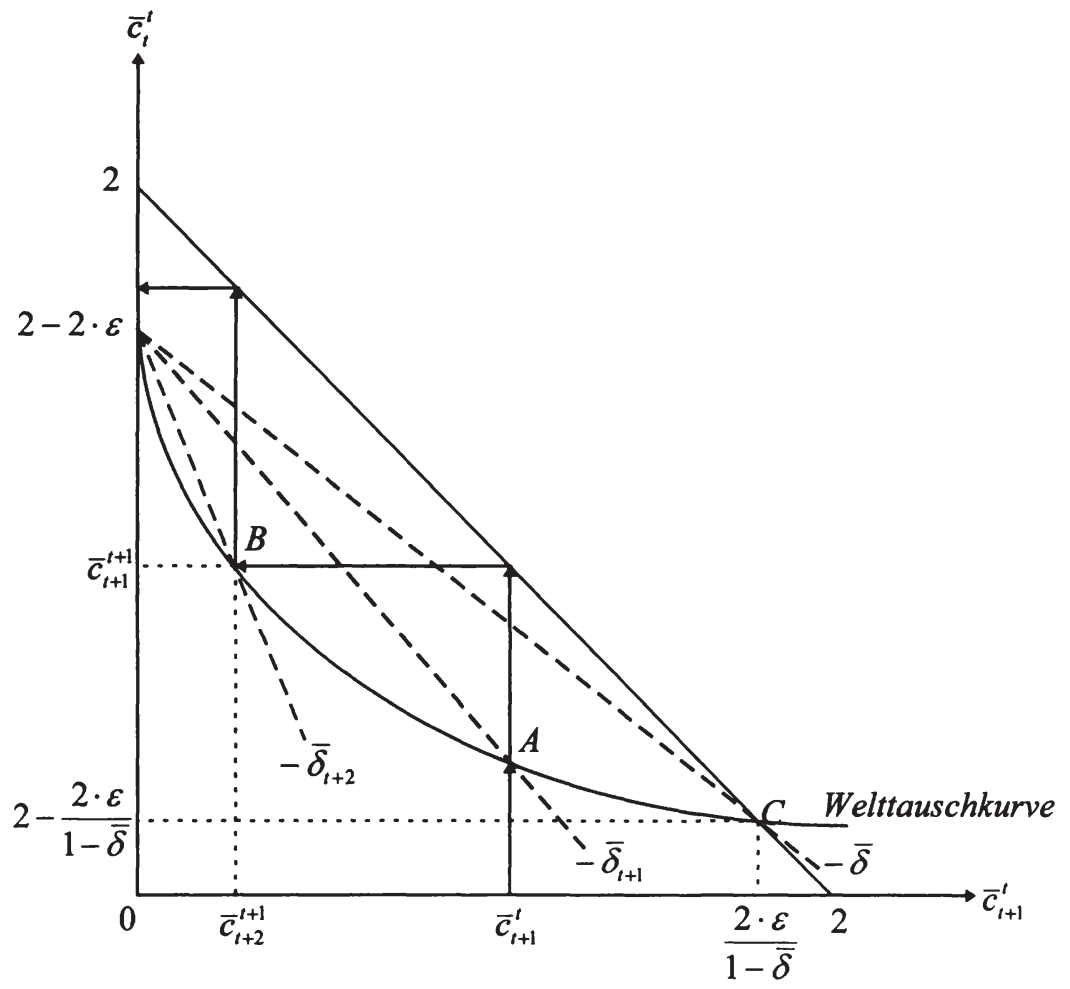

Darst. 5.2: Dynamik der integrierten Weltwirtschaft

Der aggregierte Alterskonsum der Generation $t+1 \quad \bar{c}_{t+2}^{t+1}$ ist geringer als die Summe der Dividendenzahlungen der Periode $t+2$, so daß die Anteilscheine in Periode $t+2$ einen negativen Preis $\bar{p}_{t+2}$ besitzen. ${ }^{1}$ Die Tatsache, daß nach abzählbar vielen Perioden die Weltwirtschaft in einen Bereich gerät, in dem das

' Die Weltgütermarkträumung in Periode $t+2$ würde erfordern, daß sich Generation $t+2$ in dieser Periode bei Generation $t+1$ mit $2 \cdot \varepsilon-\bar{c}_{t+2}^{t+1}$ Gütereinheiten verschuldet. Diese Schuld könnte jedoch nie getilgt werden, da Generation $t+1$ in Periode $t+3$ nicht mehr lebt. Eine alternative Interpretation wäre die, daß Generation $t+1$ der Generation $t+2$ die Gütereinheiten schenkt. Dies würde aber der Nutzenmaximierungshypothese widersprechen, da die Güter nutzenstiftend sind. 
Anrecht auf zukünftigen Konsum einen negativen Preis besitzt, schließt eine solche Entwicklung als Gleichgewichtspfad aus. ${ }^{1}$

Es wird ersichtlich, daß die integrierte Weltwirtschaft genau einen Gleichgewichtspfad besitzt, der durchführbar ist. Er ist mit dem stationären Weltkonsumplan $C$ und dem über die Zeit konstanten Diskontfaktor $\bar{\delta}$ verbunden. Die einzig mögliche Gleichgewichtsdynamik ist die, daß sich die Weltwirtschaft unmittelbar im langfristigen Gleichgewicht $C$ befindet. ${ }^{2}$ Alle anderen Pfade verlassen nach abzählbar vielen Perioden den durchführbaren Bereich. Aus Beziehung (5.5) folgt $\bar{\delta}=\bar{p} /(\bar{p}+\varepsilon) \Leftrightarrow \bar{p}=\bar{\delta} \cdot \varepsilon /(1-\bar{\delta})$.

Der Weltkonsum der Jungen im langfristigen Gleichgewicht beträgt somit

$$
\bar{c}_{t}^{t}(\bar{\delta}, 1-\varepsilon, 1-\varepsilon)=\underbrace{2-2 \cdot \varepsilon}_{\begin{array}{c}
\text { aggregiertes } \\
\text { Arbeilseinkommen }
\end{array}}-\underbrace{\bar{p} \cdot 2}_{\begin{array}{c}
\text { Kurswert } \\
\text { Aneilscheine }
\end{array}}=2-2 \cdot \varepsilon /(1-\bar{\delta}) \text {. }
$$

Der Weltkonsum der Alten im langfristigen Gleichgewicht beträgt

$$
\bar{c}_{t+1}^{\prime}(\bar{\delta}, 1-\varepsilon, 1-\varepsilon)=\underbrace{2 \cdot \varepsilon}_{\text {Dividenden }}+\underbrace{\bar{p} \cdot 2}_{\substack{\text { Kurswert } \\ \text { Anteilscheine }}}=2 \cdot \varepsilon /(1-\bar{\delta}) .
$$

Der Weltjugendkonsum wird durch die Differenz zwischen dem aggregierten Arbeitseinkommen und dem Kurswert des aggregierten Anteilscheinbestandes gegeben. Der Weltalterskonsum ist gleich der Summe der Dividendenzahlungen plus dem Kurswert des aggregierten Anteilscheinbestandes. Die aggregierte

\footnotetext{
1 Voraussetzung für diese Schlußfolgerung ist, daß sich die Akteure dadurch besser stellen können, daß sie die Vermögenstitel einfach wegwerfen. Ist diese Möglichkeit nicht gegeben, sind Gleichgewichtspfade mit negativen Preisen möglich; siehe z.B. Brock (1990), S. 27, oder auch Kehoe et al. (1991), S. 21ff.

2 Auch wenn der Diskontfaktor in einer beliebigen Periode geringer als im langfristigen Gleichgewicht ist und der Preis der Anteilscheine in dieser Periode damit höher als im langfristigen Gleichgewicht, verläßt der Gleichgewichtspfad nach abzählbar vielen Perioden den durchführbaren Bereich. Der Kurswert der Anteilscheine nimmt ständig zu, so daß er nach abzählbar vielen Perioden das Weltarbeitseinkommen der jungen Akteure übertrifft, die sie dann nicht mehr erwerben können.
} 
Gleichgewichtsallokation der integrierten Weltwirtschaft ist $(2-2 \cdot \varepsilon /(1-\bar{\delta})$, $2 \cdot \varepsilon /(1-\bar{\delta}))$.

\subsection{Die langfristigen Auswirkungen von}

\section{Außenhandel}

In diesem Abschnitt sollen die langfristigen Auswirkungen der Integration der beiden Ökonomien in eine Weltwirtschaft auf den gleichgewichtigen Konsumvektor der Akteure, sowie auf ihre Wohlfahrt und auf den Zinssatz untersucht werden. Im Spezialfall, daß $\delta=\Delta$ gilt, hat die Öffnung der Ökonomien keine Auswirkungen auf den Konsum und die Wohlfahrt in den beiden Ländern. Es gilt $\delta=\Delta=\bar{\delta}$, und damit bewirkt die Öffnung der Ökonomien keine Änderung im Verhalten der Inländer und Ausländer. Sei nun der Fall von Darstellung 5.1 betrachtet, also Ausländer mit höherer Zeitpräferenz als Inländer und damit $\delta>\Delta$. Man erkennt, daß der Diskontfaktor in der integrierten Weltwirtschaft immer zwischen den Diskontfaktoren in den einzelnen Länder liegt. Es gilt also im betrachteten Fall $\delta>\bar{\delta}>\Delta$. Somit sehen sich die Akteure des Landes, in dem in Autarkie ein höherer Zinssatz als im anderen Land vorliegt, durch die weltwirtschaftliche Integration einer Situation mit geringerer Verzinsung ihrer Ersparnis gegenüber. Das Umgekehrte gilt für die Akteure des Landes, das in Autarkie einen niedrigeren Zinssatz aufweist. Durch die Integration erhöht sich die Verzinsung ihrer Ersparnis. Argumentiert man in Gegenwartspreisen, so bedeutet dies, daß für die Akteure des einen Landes der Zukunftskonsum durch die Integration teurer wird (aus ihrer Sicht steigt der Diskontfaktor) und für die Akteure des anderen Landes billiger (aus ihrer Sicht sinkt der Diskontfaktor). ${ }^{1}$

In der betrachteten Weltwirtschaft gilt $\delta>\bar{\delta}>\Delta$ bzw. $r<\bar{r}<R$ und damit $p>\bar{p}>P$. Wie man aus Darstellung 5.1 erkennt, wird der genaue Wert von $\bar{\delta}$, $\bar{r}$ und $\bar{p}$ durch den Verlauf der Welttauschkurve determiniert. Die Form der

\footnotetext{
${ }^{1}$ Im betrachteten Fall bedeutet dies: Die Verzinsung der Ersparnis der Inländer steigt, für sie wird der Zukunftskonsum billiger; die Verzinsung der Ersparnis der Ausländer sinkt, für sie wird der Zukunftskonsum teurer.
} 
Welttauschkurve wird durch die der nationalen Tauschkurven bestimmt. Im Sonderfall, daß der Preis der Vermögenstitel bei Freihandel gleich dem arithmetischen Mittel der Autarkiepreise ist, unterscheiden sich der Weltjugendkonsum und der Weltalterskonsum in der integrierten Weltwirtschaft nicht vom Weltjugendkonsum und Weltalterskonsum bei Autarkie.

Bei $\bar{p}=(p+P) / 2$ lautet der Weltjugendkonsum:

$$
\begin{array}{ll}
\underbrace{1-\varepsilon-p}_{c^{\prime}(\delta, 1-\varepsilon)}+\underbrace{1-\varepsilon-P}_{C_{\prime}^{\prime}(\Delta, 1-\varepsilon)}=2-2 \cdot \varepsilon-(p+P) & \text { (Autarkie), } \\
\underbrace{2-2 \cdot \varepsilon-\bar{p} \cdot 2}_{\bar{c}_{\prime}^{\prime}(\tilde{\delta}, 1-\varepsilon, 1-\varepsilon)}=2-2 \cdot \varepsilon-(p+P) & \text { (Freihandel). }
\end{array}
$$

Der Weltalterskonsum ist:

$$
\begin{array}{ll}
\underbrace{\varepsilon+p}_{c_{t+1}^{\prime}(\delta, 1-\varepsilon)}+\underbrace{\varepsilon+P}_{C_{t+1}^{\prime}(\Delta, 1-\varepsilon)}=2 \cdot \varepsilon+p+P & \\
\underbrace{2 \cdot \varepsilon+\bar{p} \cdot 2}=2 \cdot \varepsilon+p+P & \text { (Futarkie) },
\end{array}
$$

Ist der Freihandelspreis der Vermögenstitel gleich dem arithmetischen Mittel der Autarkiepreise, dann lauten der Freihandelszinssatz bzw. Freihandelsdiskontfaktor wie folgt:

$$
\begin{gathered}
\bar{p}=\frac{p+P}{2} \Leftrightarrow \frac{\varepsilon}{\bar{r}}=\frac{\frac{\varepsilon}{r}+\frac{\varepsilon}{R}}{2} \quad \Leftrightarrow \quad \bar{r}=\frac{2 \cdot r \cdot R}{r+R}, \\
\bar{p}=\frac{p+P}{2} \Leftrightarrow \frac{\bar{\delta} \cdot \varepsilon}{1-\bar{\delta}}=\frac{\frac{\delta \cdot \varepsilon}{1-\delta}+\frac{\Delta \cdot \varepsilon}{1-\Delta}}{2} \Leftrightarrow \bar{\delta}=\frac{\delta+\Delta-2 \cdot \delta \cdot \Delta}{2-\delta-\Delta} .
\end{gathered}
$$


Gilt $\bar{p}>(p+P) / 2 \Leftrightarrow \bar{r}<2 \cdot r \cdot R /(r+R) \Leftrightarrow \bar{\delta}>(\delta+\Delta-2 \cdot \delta \cdot \Delta) /(2-\delta-\Delta)$, ist der Weltjugendkonsum bei Freihandel geringer als bei Autarkie, und der Weltalterskonsum ist höher. Im umgekehrten Fall ist der Weltjugendkonsum bei Freihandel höher als bei Autarkie, und der Weltalterskonsum ist geringer.

\subsubsection{Die langfristige Handelsstruktur}

Wie sehen nun die Handelsbeziehungen im langfristigen Gleichgewicht der Weltwirtschaft aus? Zur Beantwortung dieser Frage sei folgende Darstellung betrachtet.

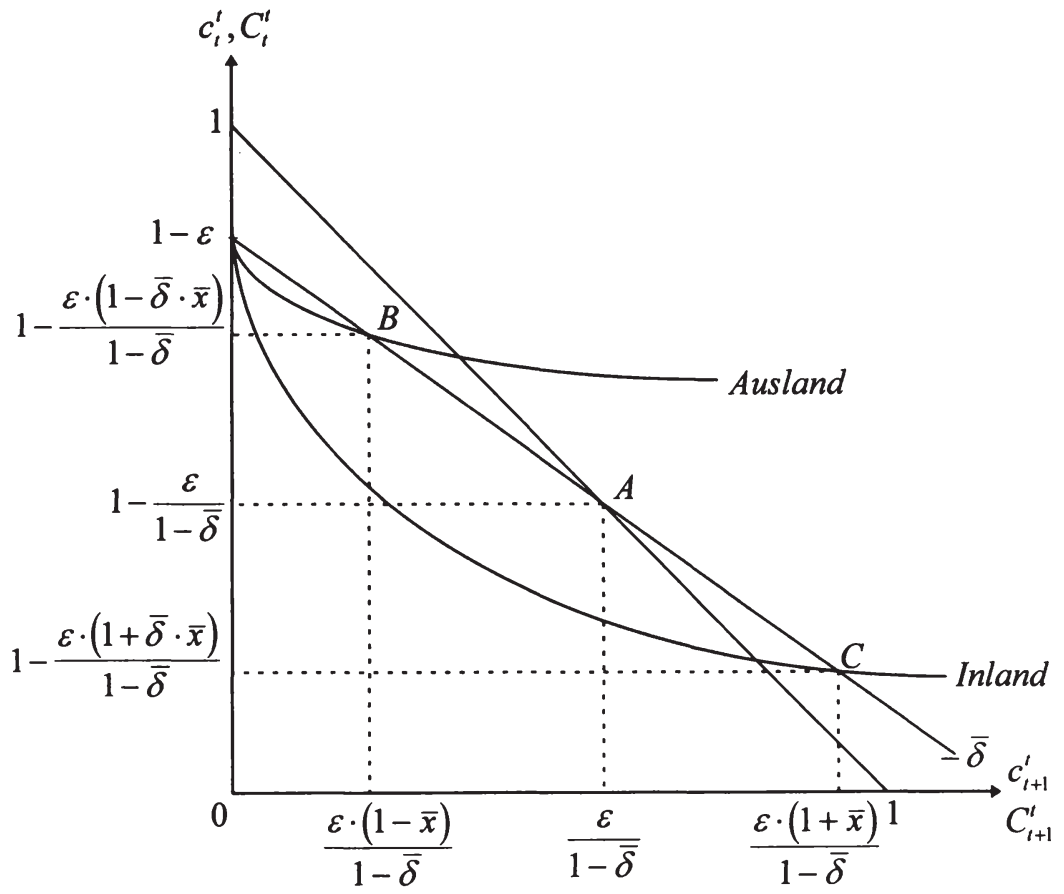

Darst. 5.3: Welthandelsgleichgewicht 
In Darstellung 5.3 werden die nationalen Konsumvektoren im langfristigen Gleichgewicht der integrierten Weltwirtschaft, unter Verwendung der nationalen Tauschkurven, hergeleitet. $\mathrm{Da}$ die Ausländer eine höhere Zeitpräferenz als die Inländer besitzen, muß das Verhältnis von Konsummenge in der Jugend und Konsummenge im Alter im Inland niedriger sein als im Ausland. Bei der auf dem Weltmarkt determinierten Verzinsung der Ersparnis sind die Inländer bereit in ihrer Jugend mehr zu sparen, als die Ausländer. Der Schnittpunkt (genannt $A$ ) der durch den Einkommenspunkt gehenden Geraden (mit der Steigung $-\bar{\delta}$ ) mit der Diagonalen gibt die langfristigen nationalen Konsumpläne an, wären die nationalen Produktionstechniken vollständig Eigentum der Akteure der betreffenden Länder. Wie man sieht, ist eine solche Allokation kein Gleichgewicht der Weltwirtschaft, da die Ausländer bei $\bar{\delta}$ in ihrer Jugend weniger sparen möchten und dafür bereit sind auf einen Teil des Konsums im Alter zu verzichten (ihr optimaler Konsumplan ist $B$ ). Das Umgekehrte gilt für die Inländer (ihr optimaler Konsumplan ist $C$ ). ${ }^{1}$ Dieses Ergebnis ist unabhängig davon, ob der Konsum in der Jugend und der Konsum im Alter Bruttosubstitute oder Bruttokomplemente sind. ${ }^{2}$

\subsubsection{Entstehung von Innengeld}

Um eine gleichgewichtige Allokation innerhalb der Weltwirtschaft zu erreichen, ist es notwendig, daß die Inländer ein Teileigentum an der ausländischen Produktionstechnik erwerben. Sie müssen also Auslandsinvestitionen tätigen und werden dadurch Anteilseigner an der ausländischen Produktionstechnik. Nur so ist es möglich, daß die inländische Ersparnis im langfristigen Gleichgewicht höher als die ausländische Ersparnis ist. Dieses optimale Sparmuster wird durch die Entstehung von Innengeld ermöglicht. ${ }^{3}$ In jeder Periode emittiert der junge

' Die optimalen nationalen Konsumpläne werden durch die Schnittpunkte der durch den Einkommenspunkt gehenden Geraden mit den nationalen Tauschkurven gegeben.

${ }^{2}$ Entscheidend ist, daß die Ausländer ungeduldiger sind als die Inländer. Bei jeder Verzinsung der Ersparnis ist das Verhältnis von optimaler Konsummenge in der Jugend und optimaler Konsummenge im Alter im Ausland höher als im Inland.

${ }^{3}$ Mit Innengeld bezeichnet man im allgemeinen durch die Akteure emittierte Schuldtitel; vgl. dazu z.B. Azariadis (1993), S. 175ff. 
ausländische Akteur einen Schuldschein, der ein Teileigentum an der ausländischen Produktionstechnik verbrieft. Die Schuldscheine werden wie die nationalen Anteilscheine ex Dividende gehandelt, so daß ein in der Periode $t$ emittierter Schuldschein einen Teilanspruch auf den Kapitalertrag (= Dividende + Verkaufserlös) in Periode $t+1$ verbrieft. Die Schuldscheine sind somit bezüglich ihrer Eigenschaft als Sparmedium vollkommene Substitute zu den nationalen Anteilscheinen, folglich haben sie den gleichen Preis (Arbitragefreiheit). Ein Anteil $\bar{x} \in(0,1]$ an der ausländischen Produktionstechnik kostet in Periode $t$ im Erwerb $\bar{x} \cdot \bar{p}_{t}$ Gütereinheiten und läßt sich in Periode $t+1$ gegen $\bar{x} \cdot\left(\bar{p}_{t+1}+\varepsilon\right)$ Gütereinheiten einlösen. Die Handelsströme im langfristigen Gleichgewicht der integrierten Weltwirtschaft lassen sich graphisch veranschaulichen. Sei hierfür Darstellung 5.4 betrachtet.

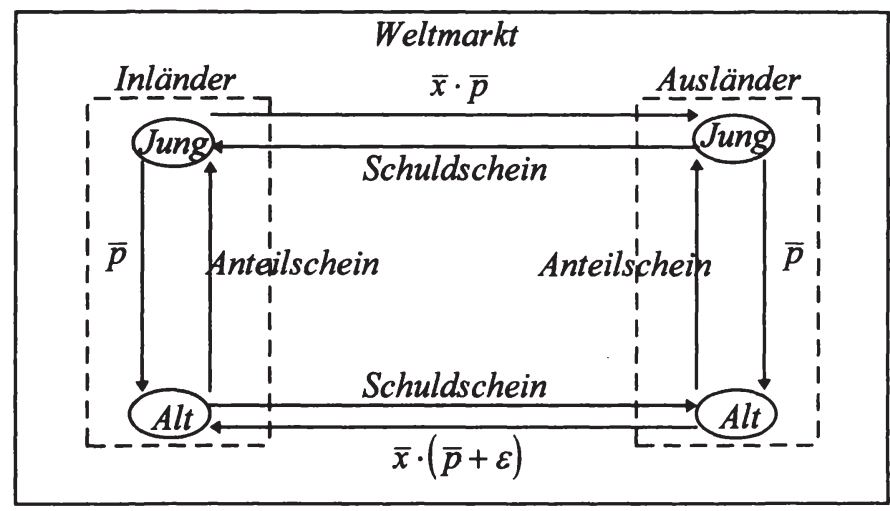

Darst. 5.4: Handelsströme

Im langfristigen Gleichgewicht der integrierten Weltwirtschaft ist der Preis der Vermögenstitel in jeder Periode $\bar{p}$. Die Alten verkaufen die Anteilscheine und erhalten dafür von den Jungen jeweils $\bar{p}$ Gütereinheiten. Dies ist die intergenerationale Komponente des Handels. Des weiteren löst der alte inländische Akteur beim alten ausländischen Akteur den Schuldschein ein, den ihm der ausländische Akteur in der vorherigen Periode ausgestellt hat. Der alte inländische Akteur erhält $\bar{x} \cdot(\bar{p}+\varepsilon)$ Gütereinheiten. Der junge ausländische Akteur stellt 
dem jungen inländischen Akteur einen Schuldschein aus, der einen Teilanpruch an dem Kapitalertrag der ausländischen Produktionstechnik in der folgenden Periode verbrieft. Für diesen Schuldschein bezahlt der junge inländische Akteur $\bar{x} \cdot \bar{p}$ Gütereinheiten. Der Handel mit den Schuldscheinen stellt die intragenerationale und zugleich internationale Komponente des Handels dar. ${ }^{1}$

\subsubsection{Die Handelsbilanz}

In jeder Periode werden im Inland somit effektiv $1+\bar{x}$ Anteilscheine und im Ausland effektiv $1-\bar{x}$ Anteilscheine gehalten, wobei $\bar{x} \in(0,1]$. Der Anteil $\bar{x}$ an der ausländischen Produktionstechnik stellt die Nettoauslandsinvestition des Inlands im langfristigen Gleichgewicht der integrierten Weltwirtschaft dar. Das Inland ist somit ein Gläubigerland (die Anzahl der von den Inländern gehaltenen Anteilscheine ist größer als die mit der inländischen Produktionstechnik verbundene), und das Ausland ist ein Schuldnerland (die Anzahl der von den Ausländern gehaltenen Anteilscheine ist geringer als die mit der ausländischen Produktionstechnik verbundene). Somit partizipiert das Inland in jeder Periode an im Ausland erzielten Gewinn. Diese Gewinnbeteiligung entspricht dem Handelsbilanzüberschuß des Auslands im langfristigen Gleichgewicht der integrierten Weltwirtschaft. Dieser ist $\bar{x} \cdot \varepsilon(=\bar{x} \cdot(\bar{p}+\varepsilon)-\bar{x} \cdot \bar{p})$. Es fällt auf, daß die Auslandsinvestitionen nicht in der Gleichgewichtsbedingung für den Weltkapitalmarkt erscheinen. Dies ist aber verständlich, da Auslandsinvestitionen nichts am aggregierten Vermögensbestand der Weltwirtschaft verändern, dieser wird durch die exogen vorgegebene Produktionstechnik determiniert.

\footnotetext{
${ }^{1}$ Es sei noch eine Anmerkung zur hergeleiteten Entstehung von Innengeld gemacht: Die Entstehung von Innengeld resultiert in der hier modellierten Weltwirtschaft aus der Tatsache, daß der Eigentumsanspruch an den nationalen Produktionstechniken von jeweils einem Anteilschein verbrieft wird. In realen Ökonomien emittieren Aktiengesellschaften eine große Anzahl von Anteilscheinen. Wollen Akteure am wirtschaftlichen Erfolg einer ausländischen Aktiengesellschaft partizipieren, erwerben sie einfach Aktien der betreffenden Gesellschaft. Der ökonomische Prozeß, der hinter dem Erwerb der Aktien bzw. dem Erwerb des Schuldscheins steckt, ist der gleiche. Es wird ein Teilanspruch am sich in der Dividendenausschüttung und der Kurswertentwicklung manifestierenden Kapitalertrag erworben.
} 
Das Modell zeigt, daß ein Außenhandelsgleichgewicht keine notwendige Eigenschaft eines langfristigen Gleichgewichts einer Weltwirtschaft miteinander handelnder Länder ist. Dieses Ergebnis stimmt mit den Resultaten aus Gale $(1971,1974)$ überein. In Gale (1971) wird im Inland und im Ausland das gleiche Gut mit den Faktoren Kapital und Arbeit produziert. Liegt die als konstant angenommene Sparquote (= der Anteil am Arbeitseinkommen, der gespart wird) zwischen den nationalen Investitionsquoten (= der Anteil am Produktionsergebnis, der investiert wird), so existiert neben dem langfristigen Gleichgewicht mit ausgeglichener Handelsbilanz ein weiteres langfristiges Gleichgewicht mit einem Handelsbilanzungleichgewicht. ${ }^{1}$ In Gale (1974) wird in einem TauschOLG-Modell gezeigt, daß wenn der Zinssatz im langfristigen Gleichgewicht der integrierten Weltwirtschaft positiv ist, das Land mit dem niedrigeren Autarkiezinssatz ein Handelsbilanzdefizit besitzt und das Land mit dem höheren Autarkiezinssatz einen Handelsbilanzüberschuß. ${ }^{2}$ Dies entspricht den Ergebnissen des hier entwickelten Modells. ${ }^{3}$

Auch im Zwei-Länder-Modell vom Diamond-Typ können langfristige Außenhandelsungleichgewichte auftreten. Buiter (1981) zeigt in einem Modell, in dem sich die beiden Länder nur durch die Zeitpräferenzrate der in ihr lebenden Akteure unterscheiden, daß der Saldo der Handelsbilanz von der relativen Zeitpräferenz abhängt und davon, ob die integrierte Weltwirtschaft sich langfristig in einem dynamisch effizienten Zustand (der Zinssatz ist hier mindestens so hoch wie die Wachstumsrate der Weltbevölkerung) oder in einem dynamisch ineffizienten Zustand (der Zinssatz ist hier niedriger als die Wachstumsrate der Weltbevölkerung) befindet. ${ }^{4}$ Auch im Diamond-Modell ist das Land, dessen

\footnotetext{
${ }^{1}$ Vgl. Gale (1971), S. 144f.

${ }^{2}$ Vgl. Gale (1974), S. 133.

${ }^{3}$ Auch das von der Produktionsseite her zum hier entwickelten Modell formal äquivalente Modell von Kareken und Wallace (1977) weist die Eigenschaft auf, daß wenn die nationalen Diskontfaktoren nicht übereinstimmen die Handelsbilanz ein Ungleichgewicht aufweist (dies gilt allerdings nur im langfristigen Gleichgewicht, in dem ein freier Handel von Gütern und Vermögenstiteln möglich ist, also im hier angenommenen Laissez-Faire-Regime); vgl. Kareken und Wallace (1977), S. 32. Das Modell von Kareken und Wallace ist in dem Sinne formal äquivalent zum vorliegenden Modell, da dort das Produktionsergebnis mit zwei Produktionsfaktoren erzeugt wird, von denen einer Arbeit ist und der andere ein nicht reproduzierbarer Produktionsfaktor (Boden).

${ }^{4}$ Vgl. Buiter (1981), S. 783ff.
} 
Akteure geduldiger sind, langfristig ein Gläubigerland und das Land, dessen Akteure eine höhere Zeitpräferenz besitzen, langfristig ein Schuldnerland. Ist das langfristige Gleichgewicht der Weltwirtschaft dynamisch effizient, so besitzt das Gläubigerland ein Handelsbilanzdefizit. Ist das langfristige Gleichgewicht dynamisch ineffizient, dann besitzt das Gläubigerland einen Handelsbilanzüberschuß. ${ }^{1}$

\subsubsection{Die langfristigen nationalen Konsumpläne}

Im langfristigen Gleichgewicht der Weltwirtschaft gilt $\bar{\delta}=\bar{p} /(\bar{p}+\varepsilon) \Leftrightarrow \bar{p}=$ $\bar{\delta} \cdot \varepsilon /(1-\bar{\delta})$. Mit dieser Kenntnis lassen sich die gleichgewichtigen nationalen Konsumvektoren des langfristigen Gleichgewichts berechnen. Für die Inländer gilt:

$$
\begin{aligned}
& c_{t}^{t}(\bar{\delta}, 1-\varepsilon)=1-\varepsilon-\bar{p} \cdot(1+\bar{x})=1-\varepsilon-\frac{\bar{\delta} \cdot \varepsilon}{1-\bar{\delta}} \cdot(1+\bar{x})=1-\frac{\varepsilon \cdot(1+\bar{\delta} \cdot \bar{x})}{1-\bar{\delta}}, \\
& c_{t+1}^{\prime}(\bar{\delta}, 1-\varepsilon)=(\bar{p}+\varepsilon) \cdot(1+\bar{x})=\left(\frac{\bar{\delta} \cdot \varepsilon}{1-\bar{\delta}}+\varepsilon\right) \cdot(1+\bar{x})=\frac{\varepsilon \cdot(1+\bar{x})}{1-\bar{\delta}}
\end{aligned}
$$

Der gleichgewichtige Konsumvektor der Ausländer ergibt sich aus

\footnotetext{
${ }^{1} \mathrm{Daß}$ die Handelsbilanz eines Gläubigerlandes einen Überschuß aufweisen kann, hängt damit zusammen, daß die Bevölkerung im Diamond-Modell mit einer positiven Rate wächst. Im langfristigen Gleichgewicht sind die Pro-Kopf-Größen konstant, so daß die Aufrechterhaltung einer konstanten Pro-Kopf-Auslandsposition einen langfristigen Leistungsbilanzüberschuß erfordert (der Leistungsbilanzüberschuß ist die positive Differenz zwischen dem Volkseinkommen und der inländischen Absorption oder einfach die Nettoauslandsinvestition). Wächst nun die Bevölkerung mit einer Rate, die höher als der Zinssatz ist, so erfordert die Konstanz der Pro-Kopf-Auslandsposition einen Handelsbilanzüberschuß (der Handelsbilanzüberschuß ist die positive Differenz zwischen der Inlandsproduktion und der inländischen Absorption). Die Auslandsinvestitionen sind hier größer als die Faktorerträge aus dem Ausland. Siehe auch Schmid und Großmann (1986), S. 37ff, zu diesem Themenkomplex.
} 


$$
\begin{aligned}
& C_{t}^{t}(\bar{\delta}, 1-\varepsilon)=1-\varepsilon-\bar{p} \cdot(1-\bar{x})=1-\varepsilon-\frac{\bar{\delta} \cdot \varepsilon}{1-\bar{\delta}} \cdot(1-\bar{x})=1-\frac{\varepsilon \cdot(1-\bar{\delta} \cdot \bar{x})}{1-\bar{\delta}}, \\
& C_{t+1}^{t}(\bar{\delta}, 1-\varepsilon)=(\bar{p}+\varepsilon) \cdot(1-\bar{x})=\left(\frac{\bar{\delta} \cdot \varepsilon}{1-\bar{\delta}}+\varepsilon\right) \cdot(1-\bar{x})=\frac{\varepsilon \cdot(1-\bar{x})}{1-\bar{\delta}}
\end{aligned}
$$

Der inländische Konsumvektor im langfristigen Laissez-Faire-Gleichgewicht der Weltwirtschaft lautet somit $(1-\varepsilon \cdot(1+\bar{\delta} \cdot \bar{x}) /(1-\bar{\delta}), \varepsilon \cdot(1+\bar{x}) /(1-\bar{\delta}))$ und der ausländische $(1-\varepsilon \cdot(1-\bar{\delta} \cdot \bar{x}) /(1-\bar{\delta}), \varepsilon \cdot(1-\bar{x}) /(1-\bar{\delta})) .{ }^{1}$ Die nationalen Konsumvektoren addieren sich zum weiter oben hergeleiteten Weltkonsumvektor $(2-2 \cdot \varepsilon /(1-\bar{\delta}), 2 \cdot \varepsilon /(1-\bar{\delta}))$.

In jeder Periode exportiert das Inland $\bar{x} \cdot \bar{p}=\varepsilon \cdot \bar{\delta} \cdot \bar{x} /(1-\bar{\delta})$ Gütereinheiten und das Ausland exportiert $\bar{x} \cdot(\bar{p}+\varepsilon)=\varepsilon \cdot \bar{x} /(1-\bar{\delta})$ Gütereinheiten, so daß der Handelsbilanzüberschuß des Auslands in jeder Periode $\bar{x} \cdot \varepsilon$ Gütereinheiten beträgt. Dieser ist gleich der in jeder Periode anfallenden Gewinnbeteiligung des Inlands an der ausländischen Produktionstechnik.

Allgemein erhöht sich durch die Integration der Weltwirtschaft der Konsum der alten Akteure in dem Land, in dem bei Autarkie ein höherer Diskontfaktor als der auf dem Weltmarkt determinierte herrscht. Das Umgekehrte gilt in dem Land, in dem der Diskontfaktor bei Autarkie niedriger ist. Dieser eindeutige Effekt resultiert aus der Normalgut-Annahme, die gewährleistet, daß der EE und der ISE einer Diskontsatzänderung im Alter gleichgerichtet wirken. Durch die weltwirtschaftliche Integration senkt sich der Diskontfaktor im einen Land (im vorliegenden Modell im Inland). Der EE und der ISE wirken in Richtung einer Erhöhung des Alterskonsums. Im anderen Land erhöht sich der Diskontfaktor durch die Integration (im vorliegenden Modell im Ausland). Der EE und der ISE wirken in diesem Land in Richtung einer Senkung des Alterskonsums. Die Auswirkung auf den Konsum in der Jugend hängen nun davon ab, ob der Konsum in der Jugend und der Konsum im Alter in den einzelnen Ländern Bruttosubstitute oder Bruttokomplemente sind. In dem Land, in dem durch die Aufnahme von Außenhandel der Diskontfaktor sinkt, erhöht sich der Konsum in der

\footnotetext{
${ }^{1}$ Siehe dazu nochmals Darstellung 5.2.
} 
Jugend bei Bruttokomplementen und sinkt bei Bruttosubstituten. Im ersten Fall überkompensiert der positive EE den ISE in der Jugend, und im zweiten Fall ist der ISE stärker. In dem Land, in dem sich durch die Aufnahme von Außenhandel der Diskontfaktor erhöht, sinkt der Konsum in der Jugend bei Bruttokomplementen und erhöht sich bei Bruttosubstituten. Im ersten Fall überkompensiert der negative EE den ISE in der Jugend, und im zweiten Fall ist der ISE stärker.

\subsubsection{Die langfristige Auswirkung auf die Wohlfahrt}

Wie wirkt sich aber nun die Integration der Weltwirtschaft auf die Wohlfahrt der Akteure in den beiden Ländern aus? Zur Beantwortung dieser Frage sei Darstellung 5.5 betrachtet. Dort sind sowohl die langfristigen Konsumpläne der Inländer als auch die der Ausländer, die sie bei Autarkie und in der integrierten Weltwirtschaft wählen, eingezeichnet. $a$ ist der Autarkiekonsumplan der Ausländer und $c$ jener der Inländer. $b$ ist der Konsumplan der Ausländer in der integrierten Weltwirtschaft und $d$ jener der Inländer. Zusätzlich sind die jeweils erreichbaren Nutzenniveaus durch Indifferenzkurven repräsentiert.

Zur Bewertung läßt sich wieder die Theorie der offenbarten Präferenz verwenden. ${ }^{1}$ Der Konsumplan $a$ wird gegenüber dem Konsumplan $b$ streng offenbart präferiert (der Wert von $b$ ist geringer als der von $a, b$ liegt innerhalb der Autarkiebudgetmenge der Ausländer und $a$ auf der Budgetgeraden, und $a$ wird gewählt). Dies impliziert, daß $a$ echt nutzenstiftender ist als $b .{ }^{2}$ Man erkennt, daß sich die Budgetmenge der ausländischen Akteure durch die Integration ge-

\footnotetext{
${ }^{1}$ Vgl. die weiter oben geführte Diskussion zu den Wohlfahrtseffekten der Staatsverschuldung und siehe auch Anhang $C$.

${ }^{2}$ Schon Fried (1980) zeigt, daß sich ein Land durch die Aufnahme von Außenhandel schlechter stellen kann; vgl. Fried (1980), S. 72ff. Fried verbindet ein OLG-Modell, in dem die beiden einzigen Produktionsfaktoren Arbeit und Boden sind, mit der Zwei-Güter-Außenhandelsstruktur des Heckscher-Ohlin-Modells (das Heckscher-Ohlin-Modell findet in der statischen Außenhandelstheorie Verwendung; siehe z.B. Ethier (1994), S. 140ff). Der Außenhandel kann die relativen Güterpreise (die intratemporalen Terms of Trade) verändern und dadurch die Faktorpreise. Dies kann einen Wohlfahrtsverlust erzeugen.
} 
genüber der Autarkiesituation verkleinert hat. Daß $u_{d}>u_{c}$ gelten muß folgt aus der Tatsache, daß sich die Budgetmenge der inländischen Akteure durch die Integration gegenüber der Autarkiesituation vergrößert hat. $\mathrm{Da}$ die inländischen Akteure in der integrierten Weltwirtschaft den Konsumplan $d$ und nicht den Konsumplan $c$ wählen, heißt nichts anderes als, daß $d$ gegenüber $c$ streng offenbart präferiert wird (der Wert von $c$ ist kleiner als der Wert von $d$, da $c$ innerhalb der Budgetmenge liegt und $d$ auf der Budgetgeraden). Dies impliziert, daß $d$ echt nutzenstiftender ist als $c$. Somit erhöht sich die Wohlfahrt der Inländer durch die weltwirtschaftliche Integration.

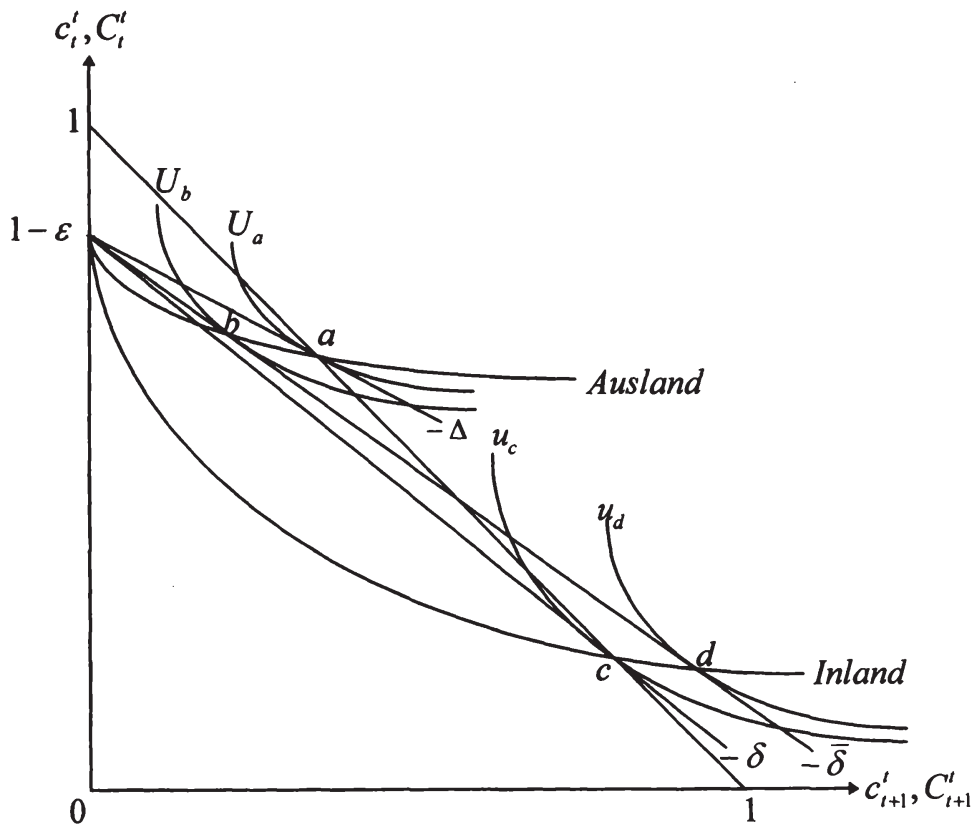

Dar. 5.5: Langfristiger Wohlfahrtseffekt von Außenhandel

Die Integration der Weltwirtschaft erzeugt durch die Zinsänderung einen Vermögenseffekt, der aus der Sicht des Auslands negativ ist (hier verkleinert sich 
durch die Reduktion des Zinssatzes die Budgetmenge der Akteure) und aus der Sicht des Inlands positiv (hier vergrößert sich durch die Erhöhung des Zinssatzes die Budgetmenge der Akteure). Die intertemporalen Terms of Trade der Inländer verbessern sich (der Zinssatz steigt aus ihrer Sicht), und die intertemporalen Terms of Trade der Ausländer verschlechtern sich (der Zinssatz sinkt aus ihrer Sicht). $\mathrm{Daß}$ sich das Ausland durch die Aufnahme von Außenhandel schlechter stellt, war schon aufgrund der Analyse der Handelsströme zu erwarten. Das Ausland besitzt in jeder Periode einen Handelsbilanzüberschuß von $\bar{x} \cdot \varepsilon$ Gütereinheiten. Somit stehen hier in jeder Periode $\bar{x} \cdot \varepsilon$ Gütereinheiten weniger zur Verfügung. Im Inland stehen in jeder Periode entsprechend $\bar{x} \cdot \varepsilon$ Gütereinheiten mehr zur Verfügung. Somit reduziert sich die Gütermenge, die im Ausland in jeder Periode zwischen dem jungen und dem alten Akteur verteilt werden kann. Im Inland erhöht sich die in jeder Periode zu verteilende Gütermenge entsprechend.

Es stellt sich nun die Frage, ob es auch im Diamond-Modell möglich ist, daß sich ein Land durch Außenhandel langfristig schlechter stellen kann. Buiter (1981) zeigt, daß es hier von entscheidender Bedeutung ist, ob das langfristige Autarkiegleichgewicht dynamisch effizient ist (der Zinssatz ist höher als die Wachstumsrate der Bevölkerung) oder dynamisch ineffizient (der Zinssatz ist niedriger als die Wachstumsrate der Bevölkerung). ${ }^{1}$ In einem dynamisch effizienten langfristigen Gleichgewicht ist die Kapitalintensität nicht höher als die der goldene Regel entsprechende. ${ }^{2}$ In einem dynamisch ineffizienten langfristigen Gleichgewicht ist die Kapitalintensität höher als die der goldene Regel entsprechende. Die Kapitalintensität und der Zinssatz in der integrierten Weltwirtschaft liegen zwischen den Autarkiewerten, wobei in dem Land mit den ungeduldigeren Akteuren der Autarkiezinssatz höher und die Kapitalintensität niedriger ist. In dem Land mit den geduldigeren Akteuren ist der Autarkiezinssatz niedriger und die Kapitalintensität höher. Es sind nun drei Fälle zu unterscheiden, wobei in der Darstellung davon ausgegangen wird, daß die Inländer geduldiger sind als die Ausländer:

\footnotetext{
${ }^{1}$ Vgl. Buiter (1981), S. 790ff.

${ }^{2}$ Eine der goldenen Regel entsprechende Kapitalintensität ermöglicht den langfristig maximalen Pro-Kopf-Konsum; vgl. z.B. Barro und Sala-i-Martin (1995), S. $19 \mathrm{ff}$.
} 
1. Die Autarkiegleichgewichte der Länder sind dynamisch ineffizient. Das Inland stellt sich durch die Integration besser. Zum einen ist die Kapitalintensität zwar immer noch über dem goldene Regel Niveau, sie ist aber gegenüber dem Autarkiezustand gesunken. Zum anderen ist das Inland ein Gläubigerland, bei einem Zinssatz, der höher als der Autarkiezinssatz ist. Das Ausland stellt sich besser oder schlechter. Wohlfahrtssteigernd wirkt sich die Tatsache aus, daß das Ausland ein Schuldnerland bei einem Zinssatz ist, der niedriger als der Autarkiezinssatz ist. Wohlfahrtsmindernd wirkt die weitere Erhöhung der Kapitalintensität.

2. Die Autarkiegleichgewichte der beiden Länder sind dynamisch effizient. Das Inland stellt sich durch die Integration nun besser oder schlechter. Wohlfahrtssteigernd wirkt sich die Tatsache aus, daß es ein Gläubigerland bei einem Zinssatz ist, der höher als der Autarkiezinssatz ist. Wohlfahrtsmindernd wirkt die Reduktion der Kapitalintensität im dynamisch effizienten Bereich. Das Ausland stellt sich besser. Zum einen erhöht sich die Kapitalintensität im dynamisch effizienten Bereich. Zum anderen ist das Ausland bei einem Zinssatz ein Schuldnerland, der unter dem Autarkiezinssatz liegt.

3. Das inländische Autarkiegleichgewicht ist dynamisch ineffizient, und das ausländische Autarkiegleichgewicht ist dynamisch effizient. Ist das langfristige Gleichgewicht der integrierten Weltwirtschaft dynamisch ineffizient, dann stellt sich das Inland besser. Die Gründe sind die gleichen wie im Fall 1. Das Ausland stellt sich besser oder schlechter. Wohlfahrtsmindernd wirkt das Ansteigen der Kapitalintensität über das dynamisch effiziente Niveau. Wohlfahrtssteigernd wirkt sich die Tatsache aus, daß das Ausland ein Schuldnerland bei einem Zinssatz ist, der niedriger als der Autarkiezinssatz ist. Ganz anders verhält es sich, wenn das langfristige Gleichgewicht der integrierten Weltwirtschaft dynamisch effizient ist. Das Inland stellt sich nun besser oder schlechter. Wohlfahrtssteigernd wirkt sich die Tatsache aus, daß es ein Gläubigerland bei einem Zinssatz ist, der höher als der Autarkiezinssatz ist. Wohlfahrtsmindernd kann sich die Reduktion der Kapitalintensität auswirken, wenn ihre negative Auswirkung im dynamisch effizienten Bereich ihre positive Auswirkung im dynamisch ineffizienten Bereich überkompensiert. Das Ausland stellt sich besser. Die Argumente sind die Gleichen wie im Fall 2. 
Die Tatsache, daß ein langfristiges Gleichgewicht im Diamond-Modell dynamisch effizient oder ineffizient sein kann, erzeugt eine Vielfalt von möglichen langfristigen Wohlfahrtseffekten der weltwirtschaftlichen Integration. Es ist also keine eindeutige Aussage wie im vorliegenden Modell möglich. In dem $\mathrm{Zu}-$ sammenhang sei auch auf die unterschiedliche Bedeutung der Auslandsinvestitionen in den beiden Modellen hingewiesen. Im hier entwickelten Modell bedeutet eine Auslandsinvestition einen Teilerwerb an der Produktionstechnik des anderen Landes. Dieser berechtigt zu einem Teilbezug der Dividende und des Verkaufserlöses. Das Arbeitseinkommen im anderen Land bleibt von der Auslandsinvestition unberührt, da sich von der Produktionsseite her nichts geändert hat. Anders im Diamond-Modell: Hier ist die Auslandsinvestition eine Direktinvestition. Sie ist mit einem Kapitalexport verbunden. Durch die Auslandsinvestition erhöht sich der Kapitalstock im anderen Land. Dies erhöht zum einen die Arbeitseinkommen, da die Kapitalintensität steigt, und reduziert die Kapitalkosten (den Zinssatz) aus dem selben Grund (es sei daran erinnert, daß eine neoklassische Produktionsfunktion unterstellt wird). Das Kapital importierende Land (das Schuldnerland) profitiert von den niedrigeren Kapitalkosten, das Kapital exportierende Land (das Gläubigerland) durch den aus seiner Sicht gestiegenen Zinssatz (das Kapital kann durch den Export einer ertragsreicheren Verwendung zugeführt werden). ${ }^{1}$

\subsection{Die Auswirkungen einer Öffnung zum Weltmarkt}

Im vorhergehenden Abschnitt wurden die langfristigen Konsequenzen von Freihandel untersucht. Im folgenden soll analysiert werden, was in der Übergangsphase zwischen Autarkiesituation und langfristigem Gleichgewicht der integrierten Weltwirtschaft passiert. Es sei also angenommen, daß das Inland und das Ausland bis zur Periode 0 autark sind, und in Periode 1 eine von den Akteuren nicht erwartete Öffnung der beiden Länder hin zum Weltmarkt stattfindet. Die in Periode 1 stattfindende Integration der beiden Länder in den Weltmarkt hat für alle folgenden Perioden Bestand, was ab der Periode 1 allen

\footnotetext{
' Vgl. dazu auch Ruffin und Yoon (1993), S. 126ff. Siehe auch Kemp und Wong (1995) für eine Diskussion der Wohlfahrtseffekte von Außenhandel im OLG-Modell.
} 
Akteuren bekannt ist. Es wird wieder der Fall des determinierten Gleichgewichtspfades betrachtet und es gilt wieder $\delta>\Delta$ und damit $\delta>\bar{\delta}>\Delta$.

Wie aus der Diskussion der Gleichgewichtsdynamik der integrierten Weltwirtschaft bekannt ist, ist der einzige durchführbare Gleichgewichtspfad der, daß sich die Weltwirtschaft von Anfang an im langfristigen Gleichgewicht befindet. Somit muß sich die Weltwirtschaft unmittelbar nach dem in Periode 1 eintretenden Strukturbruch (die Integration der beiden Länder), also ab Periode 2, im langfristigen Gleichgewicht $(2-2 \cdot \varepsilon /(1-\bar{\delta}), 2 \cdot \varepsilon /(1-\bar{\delta}))$ befinden. Damit sich die Weltwirtschaft ab Periode 2 im langfristigen Gleichgewicht befindet, muß der aggregierte Konsum der Alten in Periode 2 gleich dem aggregierten Konsum der Alten der folgenden Perioden sein, also $\bar{c}_{2}^{1}=2 \cdot \varepsilon /(1-\bar{\delta})=\bar{c}_{t+1}^{\prime}$ mit $t=2,3,4, \ldots$. Der Diskontfaktor, der gewährleistet, daß die individuellen Konsumpläne der Akteure der Generation 1 zu einem aggregierten Alterskonsum von $2 \cdot \varepsilon /(1-\bar{\delta})$ führt, ist natürlich $\bar{\delta}$. Der Preis der Vermögenstitel in Periode 2 ist $\bar{p}$, da sich die Weltwirtschaft schon ab dieser Periode im langfristigen Gleichgewicht befindet. Soll nun $\bar{\delta}_{2}=\bar{\delta}$ gelten, muß der Preis der Vermögenstitel schon in Periode $1 \bar{p}$ betragen. Somit werden die Vermögenstitel schon in Periode $1 \mathrm{zu}$ dem Preis gehandelt, den sie im langfristigen Gleichgewicht der Weltwirtschaft besitzen. Die Handelsstruktur der Periode 1 wird in Darstellung 5.6 gezeigt.

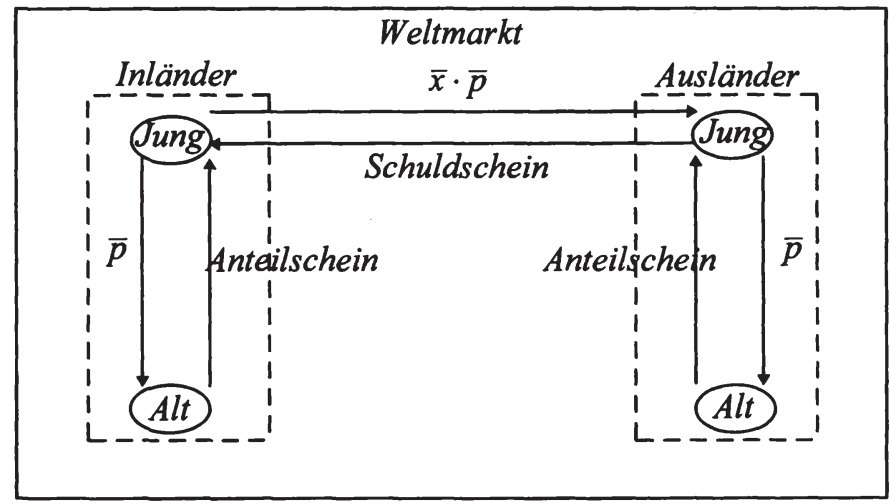

Darst. 5.6: Handelsströme in Periode 1 
Schon in Periode 1 beginnt die von da an in jeder Periode stattfindende Emission von Schuldscheinen durch die Ausländer. Nur so kann erreicht werden, daß das Aggregat der optimalen individuellen Konsumpläne der Akteure der Generation $1 \mathrm{zu}$ einem Alterskonsum in Periode 2 führt, der dem des langfristigen Gleichgewichts der Weltwirtschaft entspricht. Natürlich besteht zwischen den Akteuren der Generation 0 keine Kreditbeziehung. In Periode 0 herrschte noch der Autarkiezustand, der erst in Periode 1 überraschend aufgehoben wurde. $\mathrm{Ab}$ Periode 2 herrscht dann das aus Darstellung 5.4 bekannte Handelsmuster.

\subsubsection{Die Entwicklung der Zinssätze}

Um die Auswirkungen auf die einzelnen von der strukturellen Veränderung (Autarkie vs. Freihandel) betroffenen Generationen der beiden Länder zu eruieren, verwendet man die aus Gleichung (3.2) bekannte Aufspaltung der kurzfristigen Rendite der Vermögenstitel in den Ertrag und den Kapitalgewinn bzw. den Kapitalverlust. Da sich der inländische und der ausländische Autarkiezinssatz unterscheiden, ist es notwendig die Entwicklung aus der Sicht der Inländer und aus der Sicht der Ausländer gesondert zu analysieren.

\subsubsection{Die Entwicklung der Zinssätze aus inländischer Sicht}

Wegen $\delta>\bar{\delta}$ reduziert sich der Wert des vom inländischen Akteur 0 gehaltenen Anteilscheins in Periode 1 gegenüber der Situation, in der in Periode 1 weiterhin Autarkie herrscht. ${ }^{1}$ Es gilt $\bar{p}=\bar{\delta} \cdot \varepsilon /(1-\bar{\delta})<p=\delta \cdot \varepsilon /(1-\delta)$ und damit:

\footnotetext{
${ }^{1}$ Durch die weltwirtschaftliche Integration erhöht sich der Zinssatz aus der Sicht der Inländer langfristig. Dies ist äquivalent zu einer Reduktion des inländischen Anteilscheinpreises. Aus der Sicht der Ausländer reduziert sich der Zinssatz. Dies ist äquivalent zu einer Erhöhung des ausländischen Anteilscheinpreises (der Preis der Anteilscheine ist gleich dem mit dem langfristigen Zinssatz kapitalisierten Wert des unendlichen Dividendenstroms, somit verhalten sich Preis und Zinssatz invers zueinander).
} 
$\bar{r}_{1}=\frac{\varepsilon}{p}+\underbrace{\frac{\bar{p}-p}{p}}_{<0}<\frac{\varepsilon}{p}=r$.

Der durch die Öffnung zum Weltmarkt induzierte Preisverfall des inländischen Anteilscheins erzeugt einen Kapitalverlust, den der inländische Akteur 0 hinnehmen muß, so daß die tatsächliche Rendite seiner Ersparnis niedriger ist, als die von ihm erwartete. Es gilt $\delta>\bar{\delta}$ was äquivalent $\mathrm{zu} r<\bar{r}$ ist (der Zinssatz im langfristigen Autarkiegleichgewicht des Inlands ist niedriger als der im langfristigen Gleichgewicht der integrierten Weltwirtschaft). Aus der Sicht des Inlands ergibt sich damit folgende Entwicklung des kurzfristigen Zinssatzes: Bis Periode 0 ist der Zinssatz auf dem Niveau $r$. In Periode 1 sinkt er vorübergehend unter $r$ auf $\bar{r}_{1}$ um dann schließlich ab Periode 2 auf dem über $r$ liegenden Niveau von $\bar{r}$ zu verharren. Es gilt also $\overline{r_{1}}<r<\bar{r}$.

Um die Entwicklung der Zinsstruktur zu eruieren, verwendet man die Zinsarbitragebedingung (3.2):

$$
\begin{aligned}
& r_{0}=\frac{\varepsilon}{p}+\underbrace{\frac{p-p}{p}}_{=0}=r_{0}^{l}-\underbrace{\frac{r_{1}^{l}-r_{0}^{l}}{r_{1}^{l}}}_{=0} \Leftrightarrow r_{0}=r_{0}^{l}=r, \\
& \bar{r}_{1}=\frac{\varepsilon}{p}+\underbrace{\frac{\bar{p}-p}{p}}_{<0}=r_{1}^{l}-\underbrace{\frac{\bar{r}_{2}^{l}-r_{1}^{l}}{\bar{r}_{2}^{l}}}_{>0} \Leftrightarrow \bar{r}_{1}<r_{1}^{l}, \\
& \bar{r}_{2}=\frac{\varepsilon}{\bar{p}}+\underbrace{\frac{\bar{p}-\bar{p}}{\bar{p}}}_{=0}=\bar{r}_{2}^{l}-\underbrace{\frac{\bar{r}_{3}^{l}-\bar{r}_{2}^{l}}{\bar{r}_{3}^{l}}}_{=0} \Leftrightarrow \bar{r}_{2}=\bar{r}_{2}^{l}=\bar{r},
\end{aligned}
$$

Bis Periode 0 befindet sich das Inland im langfristigen Autarkiegleichgewicht. Hier beträgt das Zinsniveau in jeder Periode $r$, und die Zinsstruktur ist flach. In Periode 1 erzeugt die nicht antizipierte Integration in den Weltmarkt eine Reduktion des Kurswertes des inländischen Anteilscheins $(\bar{p}<p)$. Dieser Kapitalverlust spiegelt sich in einer normalen Zinsstruktur wieder $\left(\bar{r}_{1}<r_{1}^{l}\right)$. Die nor- 
male Zinsstruktur wird durch das Absinken des kurzfristigen Zinssatzes der Periode 1 unter das Zinsniveau des langfristigen inländischen Autarkiegleichgewichts erzeugt. Schon ab Periode 2 befindet sich die integrierte Weltwirtschaft im langfristigen Gleichgewicht, so daß das Zinsniveau den Wert $\bar{r}$ annimmt. Die Zinsstruktur ist ab Periode 2 wieder flach.

Die Zinsdynamik läßt sich graphisch veranschaulichen.

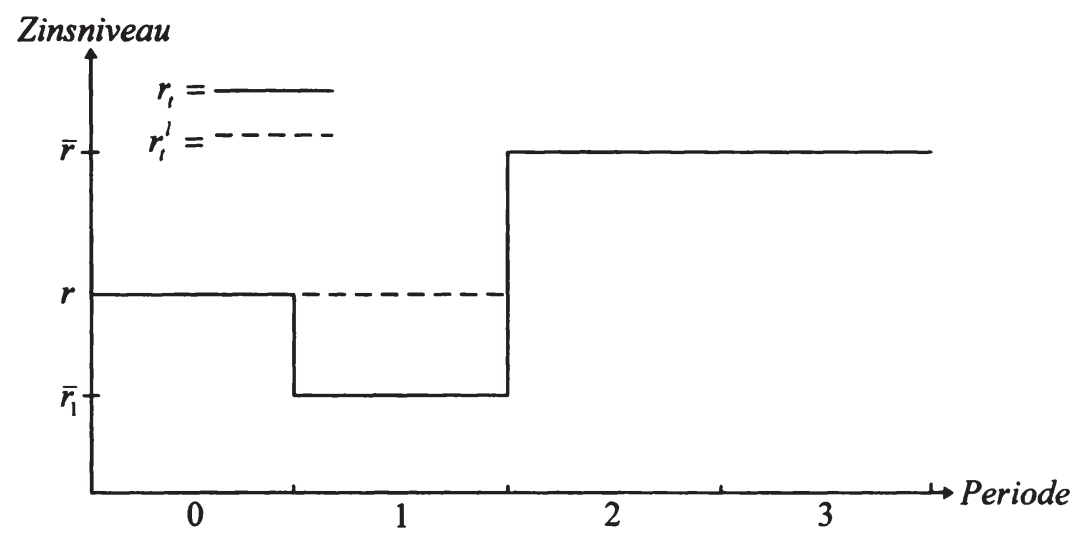

Darst. 5.7: Zinsdynamik aus inländischer Sicht

\subsubsection{Die Entwicklung der Zinssätze aus ausländischer Sicht}

Es gilt $\Delta<\bar{\delta} \Leftrightarrow P=\Delta \cdot \varepsilon /(1-\Delta)<\bar{p}=\bar{\delta} \cdot \varepsilon /(1-\bar{\delta})$ und damit

$$
\bar{R}_{1}=\frac{\varepsilon}{P}+\underbrace{\frac{\bar{p}-P}{P}}_{>0}>\frac{\varepsilon}{P}=R .
$$

Die durch die Öffnung zum Weltmarkt induzierte Kurswertsteigerung des ausländischen Anteilscheins erzeugt ein Kapitalgewinn, von dem der ausländische Akteur 0 profitiert, so daß die tatsächliche Rendite seiner Ersparnis höher als 
die von ihm erwartete ist. Es gilt $\Delta<\bar{\delta} \Leftrightarrow R>\bar{r}$ (der ausländische Zinssatz ist im langfristigen Autarkiegleichgewicht höher als im langfristigen Freihandelsgleichgewicht). Aus der Sicht des Auslands ergibt sich somit folgende Entwicklung des kurzfristigen Zinssatzes: Bis Periode 0 ist der Zinssatz auf dem Niveau $R$. In Periode 1 steigt er vorübergehend über $R$ auf $\bar{R}_{1}$ um dann schließlich ab der Periode 2 auf das unter $R$ liegende Niveau $\bar{r}$ abzusinken. Es gilt also $\bar{r}<R$ $<\bar{R}_{1}$.

Es ist klar, daß sich der kurzfristige Zinssatz der Periode 1 in den beiden Ländern unterscheiden muß (es gilt $\bar{r}_{1}<\bar{r}<\bar{R}_{1}$ ). Beide differieren nicht nur in der Ertragskomponente, sondern auch in der Kapitalgewinn- bzw. Kapitalverlustkomponente. Die Ertragskomponente ist im Inland kleiner als im Ausland $(\varepsilon / p<\varepsilon / P)$. Zusätzlich erhöht sich die Rendite im Ausland durch den auf dem Weltmarkt der Periode 1 erzeugten Kapitalgewinn bzw. reduziert sich die Rendite im Inland durch den Kapitalverlust.

Mit Hilfe der Zinsarbitragebedingung erhält man:

$$
\begin{aligned}
& R_{0}=\frac{\varepsilon}{P}+\underbrace{\frac{P-P}{P}}_{=0}=R_{0}^{l}-\underbrace{\frac{R_{1}^{l}-R_{0}^{l}}{R_{1}^{l}}}_{=0} \Leftrightarrow R_{0}=R_{0}^{l}=R, \\
& \bar{R}_{1}=\frac{\varepsilon}{P}+\underbrace{\frac{\bar{p}-P}{P}}_{>0}=R_{1}^{l}-\underbrace{\frac{\bar{R}_{2}^{l}-R_{1}^{l}}{\bar{R}_{2}^{l}}}_{<0} \Leftrightarrow \bar{R}_{1}>R_{1}^{l} \\
& \bar{R}_{2}=\frac{\varepsilon}{\bar{p}}+\underbrace{\frac{\bar{p}-\bar{p}}{\bar{p}}}_{=0}=\bar{R}_{2}^{l}-\underbrace{\frac{\bar{R}_{3}^{l}-\bar{R}_{2}^{l}}{\bar{R}_{3}^{l}}}_{=0} \Leftrightarrow \bar{R}_{2}=\bar{R}_{2}^{l}=\bar{r} .
\end{aligned}
$$

Bis Periode 0 befindet sich das Ausland im langfristigen Autarkiegleichgewicht. Hier beträgt das Zinsniveau $R$, und die Zinsstruktur ist flach. In Periode 1 erzeugt die von den Akteuren nicht antizipierte Integration der Weltwirtschaft eine Kurswertsteigerung des ausländischen Anteilscheins $(\bar{p}>P)$. Dieser Kapitalgewinn spiegelt sich in einer inversen Zinsstruktur wider $\left(\bar{R}_{1}>R_{1}^{l}\right)$. Die in- 
verse Zinsstruktur wird durch das Ansteigen des kurzfristigen Zinssatzes der Periode 1 über das Zinsniveau des langfristigen ausländischen Autarkiegleichgewichts erzeugt. Schon ab Periode 2 befindet sich die integrierte Weltwirtschaft in ihrem langfristigen Gleichgewicht, so daß das Zinsniveau den Wert $\bar{r}$ annimmt. Die Zinsstruktur ist ab Periode 2 wieder flach.

Die Zinsdynamik läßt sich wieder graphisch darstellen.

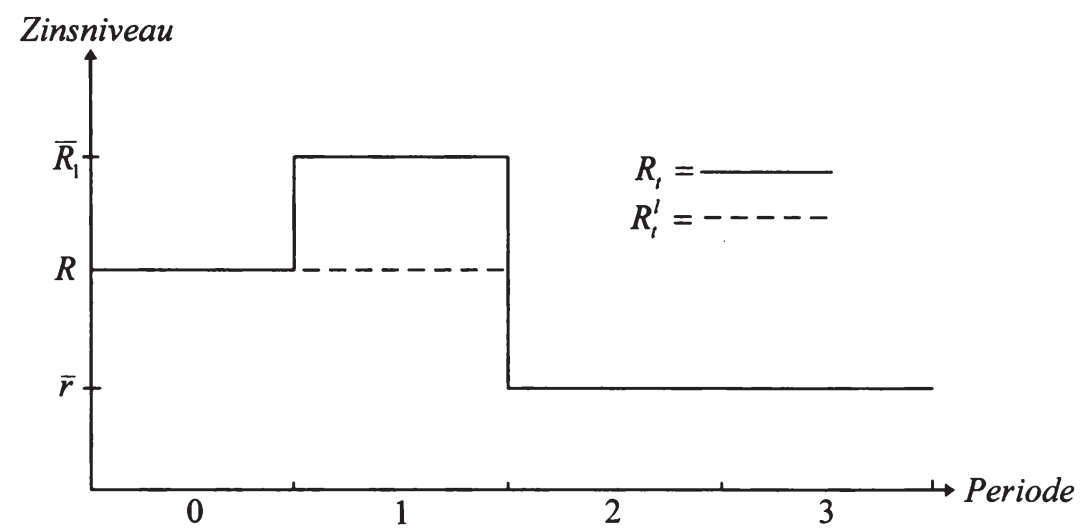

Darst. 5.8: Zinsdynamik aus ausländischer Sicht

\subsubsection{Die Auswirkung auf die Wohlfahrt}

Wie wirkt sich nun der Integrationsproze $\beta$ auf die Wohlfahrt der von ihm betroffenen Generationen aus? Generation 0 wird in Periode 1 von der unerwarteten Integration überrascht. $c_{0}^{0}$ und $C_{0}^{0}$ bleiben von der Integration unberührt, da diese nicht antizipiert wird. Der inländische Akteur 0 muß im Alter einen Kapitalverlust hinnehmen, so daß sein Konsum im Vergleich zur Autarkiesituation geringer ist $(\varepsilon+\bar{p}<\varepsilon+p)$. Seine Wohlfahrt wird somit verringert. Der ausländische Akteur 0 kommt im Alter in den Genuß eines Kapitalgewinns, so da $\beta$ sich sein Konsum im Vergleich zur Autarkiesituation erhöht $(\varepsilon+\bar{p}>\varepsilon+P)$. 
Seine Wohlfahrt erhöht sich somit. ${ }^{1}$ Genau umgekehrt sieht es mit der Wohlfahrt der folgenden Generationen aus. Wie bei der Analyse der langfristigen Auswirkungen des Außenhandels gezeigt wurde, stellt sich das Inland durch die weltwirtschaftliche Integration besser und das Ausland schlechter. ${ }^{2}$ Somit profitieren die inländischen Akteure 1, 2, 3, ... durch den Freihandel, und die ausländischen Akteure 1, 2, 3,... müssen eine Wohlfahrtsreduktion hinnehmen.

Sei nun wieder kurz das Diamond-Modell betrachtet. ${ }^{3}$ Es wird der Fall skizziert, in dem der bis zum Beginn der Öffnungsperiode akkumulierte Kapitalstock international immobil ist. Somit sind die Kapitalerträge und die Arbeitseinkommen dieser Periode prädeterminiert, und es ist irrelevant, ob die Öffnung antizipiert wurde oder nicht. Die Wohlfahrt der alten Akteure ist von der wirtschaftlichen Integration unberührt. Der im vorliegenden Modell auftretende Kapitalgewinn- und Kapitalverlusteffekt ist im Diamond-Modell nicht möglich. Die Erträge der alten Generation werden von den gegebenen nationalen Kapitalstökken determiniert. Die junge Generation des Landes mit dem niedrigeren Autarkiezinssatz profitiert von der Öffnung, und die junge Generation des Landes mit dem höheren Autarkiezinssatz wird durch die Öffnung schlechter gestellt: Nach der Öffnung fließt das Kapital vom Land mit dem niedrigeren Zinssatz in das Land mit dem höheren Zinssatz. Dies vergrößert die Konsummöglichkeiten der jungen Generation des Kapital exportierenden Landes und reduziert die Konsummöglichkeiten der jungen Generation des Kapital importierenden Landes (im ersten Land verbessern sich die intertemporalen Terms of Trade, und im zweiten Land verschlechtern sich die intertemporalen Terms of Trade). Somit profitiert das Kapital exportierende Land kurzfristig durch die Öffnung zum Weltmarkt und das Kapital importierende Land stellt sich schlechter.

Im hier entwickelten Modell sind die kurzfristigen Auswirkungen der Öffnung zum Weltmarkt zweideutig. In einem Land verliert der junge Akteur, und der

\footnotetext{
${ }^{\prime}$ Man erkennt, daß der Alterskonsum des Inländers 0 gleich dem Alterskonsum des Ausländers 0 ist. Es gilt: $c_{1}^{0}=C_{1}^{0}=\varepsilon+\bar{p}=\varepsilon /(1-\bar{\delta})$. Bedenkt man, daß die Ersparnis des Ausländers 0 geringer als die des Inländers 0 ist $\left(s^{0}=1-\varepsilon-P<S^{0}=1-\varepsilon-p\right.$, da $P>p$ ), so erkennt man, daß der Inländer 0 im Vergleich zum Ausländer 0 schlechter gestellt ist $\left(\bar{r}_{1}<\bar{R}_{1}\right)$.

${ }^{2}$ Siehe Darstellung 5.5 und die anschließende Diskussion.

${ }^{3}$ Vgl. Buiter (1981), S. 788ff.
} 
alte Akteur gewinnt, und im anderen Land gewinnt der junge Akteur, und der alte Akteur verliert. Das im hier entwickelten Modell auftretende Phänomen, daß der Zinssatz kurzfristig eine der langfristigen Entwicklung entgegengesetzte Veränderung aufweist, kann im Diamond-Modell nicht auftreten. Dort bewegt sich der Zinssatz monoton in die Richtung des langfristigen Wertes (aus der Sicht des einen Landes steigt er monoton, und aus der Sicht des anderen Landes fällt er monoton). ${ }^{1}$

${ }^{1}$ Es sei hier auch auf Eaton (1988) verwiesen. Im Modell von Eaton gibt es neben der Arbeitskraft sowohl Kapital als auch Boden als Produktionsfaktoren. Eaton untersucht nun den Einfluß der spezifischen Charakteristika der beiden Produktionsfaktoren auf die Wirkungen von Auslandsinvestitionen. Auch im Modell von Kim und Lee (1997) sind die Produktionsfaktoren Arbeit, Kapital und Boden. Kim und Lee zeigen, daß die Erhebung einer Grundsteuer zur dynamischen Ineffizienz führen kann. Bei stationärer Bevölkerungsgröße kann der Zinssatz negativ werden und bei einer wachsenden Bevölkerung kleiner als die Wachstumsrate des Bevölkerung. Crettez et al. (1998) untersuchen die langfristigen Auswirkungen der internationalen Faktormobilität auf den Zinssatz und die Wohlfahrt. Das Vorliegen eines nicht reproduzierbaren international immobilen Produktionsfaktors (Boden) bedingt, daß Kapitalmobilität und Arbeitskräftemobilität langfristig unterschiedlich wirken (die Wohlfahrtseffekte und der Zinssatz bei Kapitalmobilität unterscheiden sich von denen bei Arbeitskräftemobilität). 
Michael Ganske - 978-3-631-75472-6

Downloaded from PubFactory at 01/11/2019 04:35:58AM

via free access 


\section{Staatsverschuldung im Zwei-Länder-Modell}

In diesem Kapitel sollen die Auswirkungen von Staatsverschuldung in der integrierten Weltwirtschaft untersucht werden. Dabei interessieren zwei Fragestellungen. Unterscheiden sich die Auswirkungen der Staatsverschuldung in einer offenen Volkswirtschaft von denen in einer geschlossenen und wenn ja, inwiefern? Die Ergebnisse aus Kapital 4 dienen hierbei als Referenz. Die zweite Frage ist die, wie sich die in einem Land stattfindende staatliche Schuldenfinanzierung auf andere Länder auswirkt. Der Analyserahmen ist der aus Kapitel 5 bekannte. Es wird also eine integrierte Weltwirtschaft betrachtet, die sich dadurch auszeichnet, daß die Akteure, die das Ausland bevölkern, eine höhere Zeitpräferenz besitzen als die Inländer.

Um beide Fragekomplexe zu beantworten, ist es sinnvoll eine Weltwirtschaft zu analysieren, in der nur in einem Land eine staatliche Schuldenpolitik betrieben wird, während im anderen Land ein völlig passiver Staat unterstellt wird. Es wird darum angenommen, daß sich nur der inländische Staat verschuldet, währenddessen der Haushalt des ausländischen Staates in jeder Periode ein ausgeglichenes Budget aufweist. Bei Fragestellungen, in denen die relative Zeitpräferenz der Bevölkerung für die Ergebnisse von Bedeutung ist, wird auch der Fall diskutiert, daß sich der ausländische Staat verschuldet. Die Staatsverschuldung entsteht wie in Kapitel 4 aus einem Umverteilungsmotiv heraus. Der von der inländischen Regierung in einer Periode vorgenommene Transfer an einen inländischen Akteur erzeugt die Notwendigkeit der Emission von inländischen Staatsanleihen (vgl. Budgetrestriktion (4.1)). Die emittierten Staatsschuldverschreibungen sind wie in Kapitel 4 ewige Anleihen und eine Staatsanleihe wird in jeder Periode mit einer Couponzahlung von $\varepsilon$ Gütereinheiten verzinst. Den Zinsendienst der folgenden Perioden finanziert die inländische Regierung durch die Besteuerung der Arbeitseinkommen der folgenden inländischen Generationen. ${ }^{1} \mathrm{Da}$ die inländische Regierung nur das Arbeitseinkommen und nicht das

${ }^{1}$ Da der Staat sich über eine Lohnsteuer finanziert und nicht über eine Kapitalertragsteuer, entsteht keine Notwendigkeit ein Besteuerungsprinzip zu spezifizieren. Bei der direkten Besteuerung gibt es zwei Prinzipien der internationalen Besteuerung (vgl. Frenkel u.a. (1991), S. 22ff). Das eine ist das Wohnsitzstaatprinzip (auch Universalprinzip oder Weltprinzip ge- 
Spareinkommen der inländischen Akteure besteuert, ist die effektive Verzinsung der Ersparnis für die Inländer und die Ausländer gleich. Die Gleichgewichtsbedingung für den Weltkapitalmarkt der integrierten Weltwirtschaft mit inländischer Staatsverschuldung lautet:

$$
\begin{aligned}
\bar{s}^{t}\left(\bar{\delta}_{t+1}, 1-\varepsilon-\tau_{t}, 1-\varepsilon\right) & =\underbrace{\bar{p}_{t} \cdot\left(2+b_{t-1}\right)}_{\text {Angebot alte Generation }}+\underbrace{\bar{p}_{t} \cdot\left(b_{t}-b_{t-1}\right)}_{\text {Angebot inländischer Staat }} \\
& =\bar{p}_{t} \cdot\left(2+b_{t}\right), \forall t .
\end{aligned}
$$

Damit sich der Weltkapitalmarkt im Gleichgewicht befindet, muß die aggregierte Ersparnis der jungen Generation in jeder Periode gleich dem Kurswert der von der alten Generation und dem inländischen Staat angebotenen Vermögenstitel sein. Durch die Emission von Staatsanleihen erhöht sich der Weltbestand an Vermögenstiteln. Das aggregierte verfügbare Einkommen einer Generation reduziert sich durch die Besteuerung des inländischen Akteurs (wenn $\tau_{t}>0$ ) bzw. erhöht sich durch den ihm zukommenden Transfer (wenn $\tau_{t}<0$ ). Die aggregierte Ersparnis einer Generation wird durch die Differenz zwischen ihrem aggregierten verfügbaren Einkommen und ihrem aggregierten Jugendkonsum gegeben. Die aggregierte Konsumnachfrage der Jungen einer Periode wird durch den auf dem Weltmarkt determinierten Diskontfaktor und durch ihr verfügbares Einkommen determiniert. Wenn $\tau_{t} \neq 0$ gilt, unterscheiden sich die verfügbaren Einkommen des jungen Inländers $t$ und des jungen Ausländers $t$. Die Höhe von $\tau_{t}$ beeinflußt das verfügbare Einkommen und das Konsumver-

nannt). Nach diesem Prinzip findet die Besteuerung nach dem Wohnsitz des Steuerschuldners statt, unabhängig davon in welchem Land das zu versteuernde Einkommen entsteht. Das zweite Prinzip ist das Quellenstaatprinzip (auch Territorialprinzip genannt). Hier findet die Besteuerung in dem Land statt, in dem das Einkommen entsteht. Würde sich der Staat durch eine Kapitalertragsteuer finanzieren, wäre es notwendig ein Besteuerungsprinzip festzulegen, da das Inland im langfristigen Gleichgewicht der integrierten Weltwirtschaft ein Gläubigerland ist, und die inländischen Akteure Auslandsinvestitionen tätigen (sie erlangen ein Teileigentum an der ausländischen Produktionstechnik). Somit entsteht ein Teil der Spareinkommen der Inländer im Ausland (sie erhalten einen Teil des ausländischen Kapitalertrags). Würde die inländische Regierung die Spareinkommen nach dem Quellenstaatprinzip besteuern, wäre nur der Ertrag der inländischen Produktionstechnik Steuerobjekt. Würde die Besteuerung nach dem Wohnsitzstaatprinzip erfolgen, wäre das gesamte Spareinkommen der Inländer Steuerobjekt. Zum Wohnsitzstaatprinzip vs. Quellenstaatprinzip siehe auch Abschnitt 2.2.2.3, Bovenberg (1992), S. 275ff, Bovenberg (1994), S. 123ff, und Bianconi (1995), S. $402 \mathrm{ff}$. 
halten des Inländers $t$ und damit auch das aggregierte Sparverhalten der Generation $t$.

Da in keinem Land ein öffentlicher Verbrauch von Gütern stattfindet, erfordert die Gleichgewichtsbedingung des Weltgütermarktes weiterhin, daß die Summe aus aggregiertem Konsum der Jungen und aggregiertem Konsum der Alten in jeder Periode 2 beträgt (die Weltgüterproduktion beträgt weiterhin 2 Gütereinheiten pro Periode):

$$
\bar{c}_{t}^{t}\left(\bar{\delta}_{t+1}, 1-\varepsilon-\tau_{t}, 1-\varepsilon\right)+\bar{c}_{t}^{t-1}\left(\bar{\delta}_{t}, 1-\varepsilon-\tau_{t-1}, 1-\varepsilon\right)=2, \forall t .
$$

Nun läßt sich ein Walras-Gleichgewicht der integrierten Weltwirtschaft mit inländischer Staatsverschuldung wie folgt beschreiben:

Eine Folge $\left\{\bar{p}_{t}, \tau_{t}, b_{t},\left(c_{t}^{t}, c_{t+1}^{t}\right),\left(C_{t}^{t}, C_{t+1}^{t}\right)\right\}_{t \in \mathrm{Z}}$ beschreibt ein Walras-Gleichgewicht der integrierten Weltwirtschaft bei vollkommener Voraussicht und mit inländischer Staatsverschuldung, wenn $\left\{\tau_{t}, b_{t}\right\}_{t \in Z}$ die staatliche Budgetrestriktion (4.1) erfüllt, die nationalen Konsumpläne $\left\{\left(c_{t}^{t}, c_{t+1}^{t}\right)\right\}_{t \in \mathcal{Z}}$ bzw. $\left\{\left(C_{t}^{t}, C_{t+1}^{t}\right)\right\}_{t \in \mathcal{Z}}$ den Optimalitätsbedingungen (3.9) bzw. (5.2) genügen und der Weltkapitalmarkt sowie der Weltgütermarkt in jeder Periode geräumt werden, also die Gleichungen (6.1) und (6.2) erfüllt sind.

\subsection{Die langfristigen Auswirkungen von Staatsverschuldung}

Ein langfristiges Gleichgewicht der integrierten Weltwirtschaft zeichnet sich dadurch aus, daß die Anzahl der sich im Umlauf befindlichen inländischen Staatsanleihen über die Zeit konstant ist, und der inländische Staat seinen Zinsendienst durch eine über die Zeit konstante Besteuerung des inländischen Arbeitseinkommens bestreitet. Aus der staatlichen Budgetrestriktion (4.1) folgt $\tau=\varepsilon \cdot b$. Der Preis der Vermögenstitel und der Diskontfaktor sind im langfristigen Gleichgewicht konstant. Aus Beziehung (5.5) folgt $\bar{p}=\bar{\delta} \cdot \varepsilon /(1-\bar{\delta})$. Der 
langfristige Weltkonsumplan der integrierten Weltwirtschaft mit inländischer Staatsverschuldung ist somit:

$\bar{c}_{t}^{t}(\bar{\delta}, 1-\varepsilon-\tau, 1-\varepsilon)=\underbrace{2-2 \cdot \varepsilon-\tau}_{\begin{array}{c}\text { aggregierles } \\ \text { verfigbares Eintommen }\end{array}}-\underbrace{\bar{p} \cdot(2+b)}_{\begin{array}{c}\text { Kurswert } \\ \text { Weltvermogenstitelbest. }\end{array}}=2-\frac{\varepsilon \cdot(2+b)}{1-\bar{\delta}}$,

$\bar{c}_{t+1}^{t}(\bar{\delta}, 1-\varepsilon-\tau, 1-\varepsilon)=\underbrace{\varepsilon \cdot(2+b)}_{\begin{array}{c}\text { Dividenden } \\ \text { Coupons }\end{array}}+\underbrace{\bar{p} \cdot(2+b)}_{\begin{array}{c}\text { Kurswert } \\ \text { Welvermogensitielbest. }\end{array}}=\frac{\varepsilon \cdot(2+b)}{1-\bar{\delta}}$.

Der Weltjugendkonsum wird durch die Differenz zwischen dem um die Besteuerung der jungen Inländer reduzierten Weltarbeitseinkommen und dem Kurswert des Weltvermögenstitelbestandes gegeben. Der Weltalterskonsum ist gleich der Summe aus Coupon- und Dividendenzahlungen plus dem Kurswert des Weltvermögenstitelbestandes. Somit ist der Weltkonsumvektor im langfristigen Gleichgewicht der integrierten Weltwirtschaft mit inländischer Staatsverschuldung $(2-\varepsilon \cdot(2+b) /(1-\bar{\delta}), \varepsilon \cdot(2+b) /(1-\bar{\delta}))$.

Das Walras-Gesetz kommt wieder zur Anwendung: Die langfristigen Auswirkungen der inländischen Staatsverschuldung auf den Weltkonsumplan lassen sich durch Betrachtung des Weltgütermarktes analysieren. ${ }^{1}$ Hierbei wird, wie auch schon in den Kapiteln 4 und 5, wieder der determinierte (reguläre) Fall betrachtet. In Darstellung 6.1 sind das langfristige Gleichgewicht der integrierten Weltwirtschaft bei unverschuldeten Staaten (Situation $a$ ) und bei einem verschuldeten inländischen Staat (Situation $b$ ) eingezeichnet. Die Welttauschkurven beider Situationen erhält man entsprechend der in Darstellung 5.1 beschriebenen Prozedur. ${ }^{2}$

\footnotetext{
${ }^{1}$ Es existieren weiterhin zwei Märkte. Dies sind der integrierte Gütermarkt und der integrierte Kapitalmarkt. Auf dem Weltkapitalmarkt werden nun zusätzlich zu den nationalen Anteilscheinen und den Schuldscheinen die inländischen Staatsanleihen gehandelt. Aufgrund ihrer Ausgestaltung sind alle drei Arten von Vermögenstiteln allerdings vollkommene Substitute im Portfolio der Anleger.

2 Der Unterschied der Situation mit inländischer Staatsverschuldung zur Situation ohne Staatsschuld liegt darin, daß die nationalen Tauschkurven im ersten Fall die Ordinate nicht im gleichen Punkt schneiden. Die Aggregationsprozedur bleibt aber die gleiche. Die zu den ein-
} 


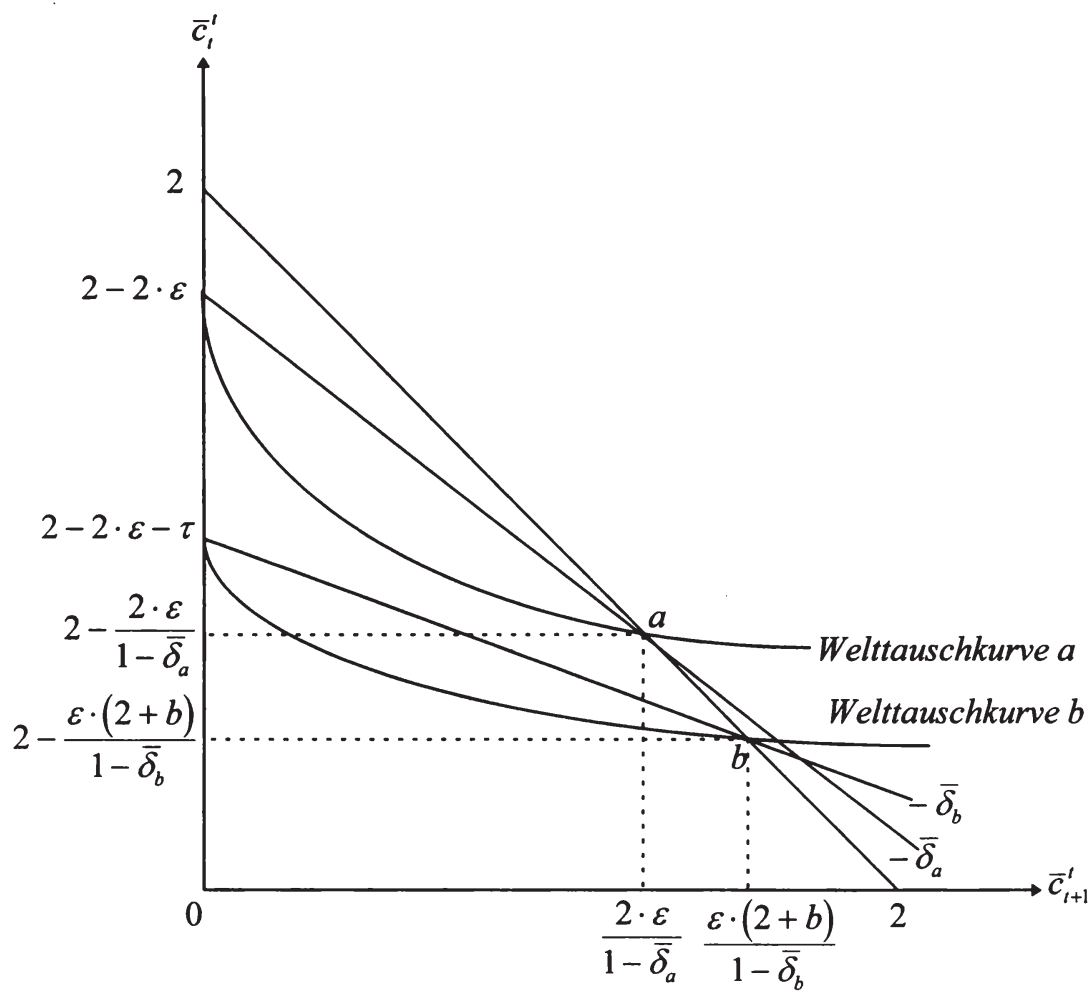

Darst. 6.1: Auswirkungen der Staatsverschuldung

Die Staatsverschuldung erzeugt, wie im folgenden Abschnitt gezeigt wird, eine Reduktion des Diskontfaktors bzw. eine Erhöhung des Zinssatzes. Dies bewirkt eine Reduktion des Weltkonsums der Jungen und eine Erhöhung des Weltkonsums der Alten. Das Argument ist das gleiche wie für die Auswirkung der Staatsverschuldung auf den Konsumplan der Akteure in der geschlossenen Ökonomie. Aufgrund der Besteuerung des inländischen Arbeitseinkommens

zelnen Diskontfaktoren gehörenden optimalen nationalen Konsumvektoren werden zur Welttauschkurve aggregiert. 
reduziert sich das aggregierte verfügbare Einkommen der Jungen. Dies bewirkt eine Reduktion der Weltnachfrage der Jungen und der Alten (NormalgutAnnahme). Die Erhöhung des Zinssatzes wirkt in Richtung einer Erhöhung des Weltkonsums der Alten. Da der Weltkonsumplan auf der die Weltgütermarktgleichgewichte beschreibenden Diagonalen liegen muß, ist die einzige mögliche Entwicklung dadurch gegeben, daß er mit steigender Verschuldung nach rechts unten wandert. ${ }^{1}$

\subsubsection{Der Zinssatz}

Wie schon im Fall der geschlossenen Ökonomie reduziert die Staatsverschuldung den Diskontfaktor bzw. erhöht den Zinssatz. Daß dies der Fall sein muß, läßt sich analytisch leicht zeigen. ${ }^{2}$ Schreibt man die Gleichgewichtsbedingung des Weltgütermarktes (6.2) in impliziter Form und berücksichtigt, daß im langfristigen Gleichgewicht $\tau=\varepsilon \cdot b$ gilt, erhält man:

$$
E(\bar{\delta}(b), b):=\bar{c}_{t}^{t}(\bar{\delta}, 1-\varepsilon-\varepsilon \cdot b, 1-\varepsilon)+\bar{c}_{t-1}^{t}(\bar{\delta}, 1-\varepsilon-\varepsilon \cdot b, 1-\varepsilon)-2=0
$$

Es gilt $d E / d b=\partial E / \partial \bar{\delta} \cdot d \bar{\delta} / d b+\partial E / \partial b$. Setzt man diesen Ausdruck gleich null, erhält man nach Umformung:

$$
\frac{d \bar{\delta}}{d b}=-\frac{\partial E / \partial b}{\partial E / \partial \bar{\delta}}
$$

\footnotetext{
${ }^{1}$ Der ISE der Erhöhung des Zinssatzes wirkt in Richtung einer Reduktion des Weltjugendkonsums und einer Erhöhung des Weltalterskonsums. Der EE wirkt in Richtung einer Erhöhung des Weltkonsums in beiden Lebensabschnitten und damit entgegengesetzt dem EE, der aus der Reduktion des aggregierten verfügbaren Einkommens resultiert. Selbst wenn der EE der Erhöhung des Zinssatzes den EE der Reduktion des verfügbaren Einkommens überkompensiert, kann es nicht sein, daß der Weltkonsumplan nach links oben wandert, da sich dann der Weltalterskonsum reduzieren würde.

${ }^{2}$ Vgl. Fried und Howitt (1988), S. 11.
} 
(6.6) ist die Differentiationsregel für implizite Funktionen (implicit-functionrule). ${ }^{1}$ Definiert man $w:=1-\varepsilon-\varepsilon \cdot b$ (= verfügbares Einkommen der jungen Inländer), dann folgt aus der intertemporalen Budgetrestriktion der Inländer bzw. der Ausländer:

$$
\begin{aligned}
& \frac{\partial c_{t}^{t}}{\partial w}+\bar{\delta} \cdot \frac{\partial c_{t+1}^{t}}{\partial w}=1 \\
& \Leftrightarrow \frac{\partial c_{t}^{t}}{\partial w}+\frac{\partial c_{t+1}^{t}}{\partial w}=1+(1-\bar{\delta}) \cdot \underbrace{\frac{\partial c_{t+1}^{t}}{\partial w}}_{>0}>0, \text { für } \bar{\delta} \leq 1, \\
& \frac{\partial c_{t}^{t}}{\partial \bar{\delta}}+\bar{\delta} \cdot \frac{\partial c_{t+1}^{t}}{\partial \bar{\delta}}+c_{t+1}^{\prime}=0 \\
& \Leftrightarrow \frac{\partial c_{t}^{t}}{\partial \bar{\delta}}+\frac{\partial c_{t+1}^{t}}{\partial \bar{\delta}}=-c_{t+1}^{t}+(1-\bar{\delta}) \cdot \underbrace{\frac{\partial c_{t+1}^{t}}{\partial \bar{\delta}}}_{<0}<0 \text {, für } \bar{\delta} \leq 1, \\
& \frac{\partial C_{t}^{t}}{\partial \bar{\delta}}+\bar{\delta} \cdot \frac{\partial C_{t+1}^{t}}{\partial \bar{\delta}}+C_{t+1}^{t}=0 \\
& \Leftrightarrow \frac{\partial C_{t}^{t}}{\partial \bar{\delta}}+\frac{\partial C_{t+1}^{t}}{\partial \bar{\delta}}=-C_{t+1}^{t}+(1-\bar{\delta}) \cdot \underbrace{\frac{\partial C_{t+1}^{t}}{\partial \bar{\delta}}}_{<0}<0, \text { für } \bar{\delta} \leq 1 \text {. }
\end{aligned}
$$

Gleichung (6.7) ist die Engel-Aggregation und Gleichung (6.8) bzw. (6.9) die Cournot-Aggregation. ${ }^{2}$ Die Engel-Aggregation manifestiert die Tatsache, daß die aus einer Veränderung des verfügbaren Einkommens resultierende Veränderung der Konsumausgaben mit der Veränderung des Einkommens übereinstimmen muß. Die Cournot-Aggregation gibt den Umstand wieder, daß sich die Konsumausgaben, bei gegebenem Einkommen, als Reaktion auf eine Preisveränderung nicht verändern können.

\footnotetext{
${ }^{1}$ Vgl. z.B. Chiang (1984), S. $207 \mathrm{ff}$.

${ }^{2}$ Siehe dazu Barten und Böhm (1982), S. 416.
} 
Die Erhöhung der inländischen Staatsschuld bedingt zum einen eine Erhöhung des Zinsendienstes, welcher durch eine erhöhte Besteuerung des Arbeitseinkommens der Inländer finanziert wird. Zum anderen wirkt die Staatsverschuldung auf den Zinssatz. Die ausländischen Akteure werden nur vom zweiten Effekt betroffen. Berücksichtigt man diesen Umstand, kann man (6.6), unter Verwendung der Definitionen (5.6), (5.7) und (6.5), wie folgt umformen:

$$
\frac{d \bar{\delta}}{d b}=\frac{\varepsilon \cdot\left(\partial c_{t}^{t} / \partial w+\partial c_{t-1}^{t} / \partial w\right)}{\partial c_{t}^{t} / \partial \bar{\delta}+\partial C_{t}^{t} / \partial \bar{\delta}+\partial c_{t-1}^{t} / \partial \bar{\delta}+\partial C_{t-1}^{t} / \partial \bar{\delta}}<0 .
$$

Im langfristigen Gleichgewicht sind die individuellen Konsummengen der Jungen und der Alten über die Zeit konstant. Der Zähler von (6.10) ist somit wegen (6.7) größer als null, und der Nenner ist wegen (6.8) und (6.9) kleiner als Null. Somit nimmt der Diskontfaktor im langfristigen Gleichgewicht der integrierten Weltwirtschaft mit zunehmender Staatsverschuldung ab. Da $\bar{p}=\bar{\delta} \cdot \varepsilon /(1-\bar{\delta})$ gilt, nimmt auch der Kurswert der Vermögenstitel mit zunehmender Staatsverschuldung ab. Qualitativ unterscheiden sich die langfristigen Auswirkungen der Staatsverschuldung auf den Zinssatz und die Preise der Vermögenstitel somit nicht von denen in einer geschlossenen Ökonomie.

Ökonomisch läßt sich das Absinken des Diskontfaktors wie folgt interpretieren: Wie aus Kapitel 4 bekannt ist, reduziert sich durch die Staatsverschuldung der Diskontfaktor im langfristigen Gleichgewicht einer geschlossenen Volkswirtschaft. Im langfristigen Gleichgewicht der integrierten Weltwirtschaft ist es ohne Bedeutung, ob die beiden Länder sich dem Außenhandel geöffnet haben und sich dann die Staatsverschuldung in einem Land erhöht hat, oder ob die Staatsschuld schon im Zeitpunkt der Öffnung vorhanden war. Im betrachteten Beispiel reduziert die inländische Staatsverschuldung den inländischen Autarkiediskontfaktor und reduziert damit den Unterschied zwischen Inland und Ausland (der Autarkiediskontfaktor des Auslands ohne Staatsverschuldung ist geringer als der des Inlands ohne Staatsverschuldung). Daher ist es nur natürlich, daß sich auch der Diskontfaktor im langfristigen Gleichgewicht der integrierten Weltwirtschaft reduziert. Im Fall, daß sich der ausländische Staat verschuldet, gilt dasselbe. Auch im Ausland würde sich in Autarkie der Diskontfaktor durch die Staatsverschuldung reduzieren. In diesem Fall wird der Unterschied zwi- 
schen dem Inland und dem Ausland aber noch akzentuiert (die Differenz der Autarkiediskontfaktoren nimmt zu).

Wie bei der Herleitung des langfristigen Gleichgewichts der integrierten Weltwirtschaft in Kapitel 5 gezeigt wurde, schneidet die Welttauschkurve diejenige Diagonale genau einmal, welche die Weltallokationen, die den Weltgütermarkt räumen, beschreibt. Weltwirtschaften mit unterschiedlichen staatlichen Verschuldungsgraden zeichnen sich durch sich unterscheidende Welttauschkurven aus, welche die Diagonale aber alle genau einmal schneiden müssen. Wie schon im Fall der geschlossenen Ökonomie, existiert für alle $b \in[0,(1-\varepsilon) / \varepsilon)$ genau ein langfristiges Gleichgewicht der integrierten Weltwirtschaft, wobei jedes dieser Gleichgewichte mit genau einem Diskontfaktor $\bar{\delta}$, mit $0<\bar{\delta}<1$, verbunden ist. $^{1} \bar{\delta}$ ist hierbei eine injektive Funktion von $b$, also $\bar{\delta}(b)$.

\subsubsection{Die nationalen Konsumpläne}

Wie sich die inländische Staatsverschuldung auf die langfristigen nationalen Konsumvektoren auswirkt, soll mit Hilfe von Darstellung 6.2 untersucht werden. Der langfristige Konsumvektor des Auslands ist mit $a$ bezeichnet und der des Inlands mit $d$. Da der Diskontfaktor durch die inländische Staatsverschuldung gegenüber der Situation ohne Staatsverschuldung gesunken ist, erhöht sich der Lebenskonsum der ausländischen Akteure. Dies ergibt sich aus der Cournot-Aggregation (6.9), läßt sich aber auch geometrisch zeigen: Das Absinken des Diskontfaktors dreht die Budgetgerade der ausländischen Akteure gegen den Uhrzeigersins, was ihre Budgetmenge vergrößert. Aufgrund des elastischen Verlaufs der Tauschkurve vergrößert sich der Alterskonsum der Ausländer. Ihr Konsum in der Jugend sinkt (bei Bruttosubstituten) oder steigt (bei Bruttokomplementen). Die Summe aus beiden muß aber steigen (aufgrund des

\footnotetext{
${ }^{\prime}$ Auch in der integrierten Weltwirtschaft gilt, daß ein Staat seinen Akteuren durch die Besteuerung nicht das gesamte Arbeitseinkommen entziehen kann (es muß also $\tau<1-\varepsilon$ und damit $b<(1-\varepsilon) / \varepsilon$ gelten). Würde er dies nämlich tun, wäre der Autarkiezinssatz unendlich (der Preis der Vermögenstitel wäre null). In diesem Fall würde kein langfristiges Gleichgewicht der integrierten Weltwirtschaft existieren.
} 
durch die inländische Staatsverschuldung induzierten positiven Vermögenseffekts, der sich in einer Vergrößerung der Budgetmenge niederschlägt).

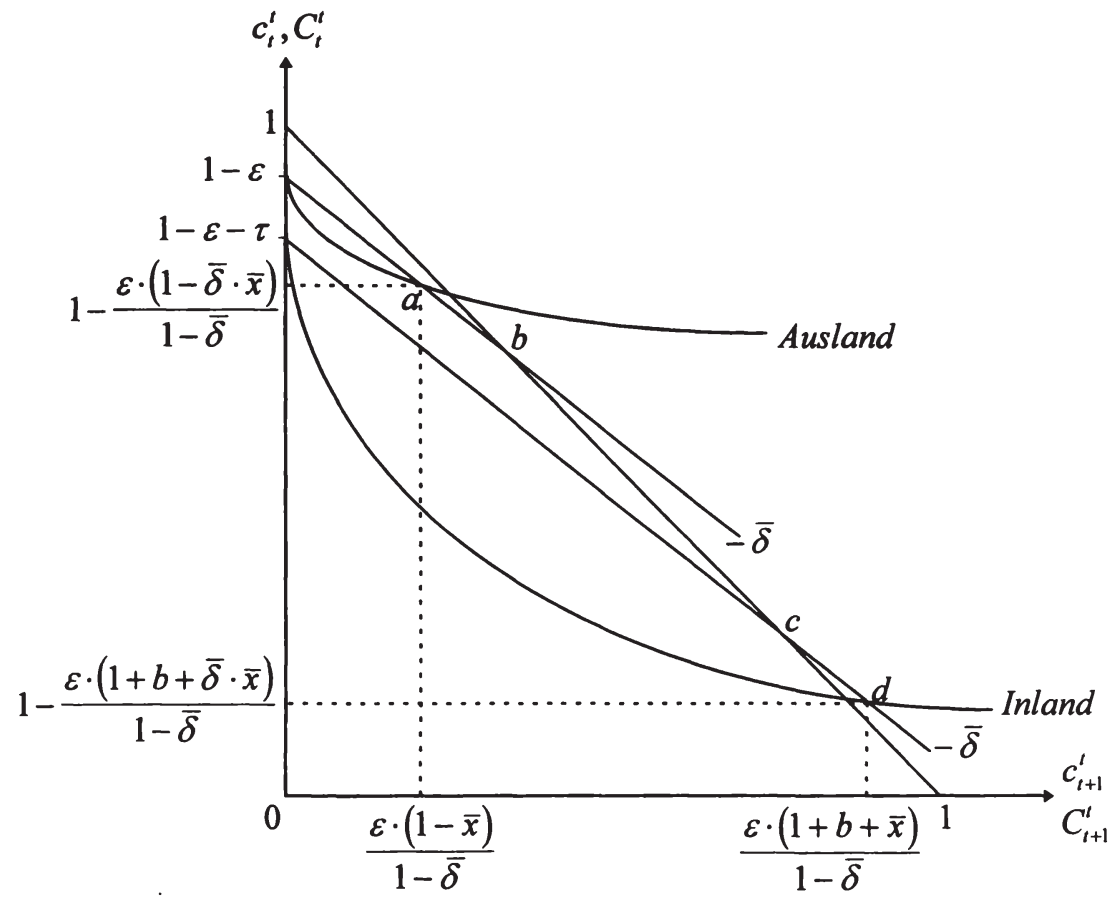

Darst. 6.2: Auswirkung auf die nationalen Konsumvektoren

Da der ausländische Konsum langfristig zunimmt, muß der inländische Konsum folglich abnehmen (Weltgütermarkträumung). Aus der Normalgut-Annahme folgt dabei, daß der Jugendkonsum der Inländer sinken muß: Das verfügbare Einkommen der Inländer sinkt durch die Besteuerung ihres Arbeitseinkommens. Dies wirkt in Richtung einer Reduktion ihres Jugendkonsums und ihres Alterskonsums (Normalgut-Annahme). Die inländische Staatsverschuldung reduziert auch den Diskontfaktor. Der ISE und der EE wirken im Alter in Richtung einer Erhöhung des Konsums, in der Jugend wirkt der EE in Richtung einer Erhöhung 
und der ISE in Richtung einer Reduktion. Der EE der Einkommensreduktion und der EE der Reduktion des Diskontfaktors wirken entgegengesetzt. Aufgrund des ISE nimmt das Verhältnis von Jugendkonsum und Alterskonsum somit ab. Da der Inlandskonsum sinkt, muß der Jugendkonsum der Inländer folglich abnehmen. Der Alterskonsum der Inländer erhöht sich oder sinkt. Die mögliche Reduktion des Alterskonsums muß allerdings geringer sein als die des Jugendkonsum, da das Verhältnis von beiden abnimmt.

Für die beschriebenen Auswirkungen ist es nicht von Bedeutung, ob sich der Staat des Gläubigerlandes oder der des Schuldnerlandes verschuldet. In beiden Fällen reduziert die Staatsverschuldung den Diskontfaktor der integrierten Weltwirtschaft. Die Akteure des Landes, in dem keine Staatsverschuldung stattfindet, sind nur durch die Reduktion des Diskontfaktors betroffen. Diese vergrößert ihre Budgetmenge und erhöht die Summe aus Jugend- und Alterskonsum (Cournot-Aggregation). Die Akteure des Landes, dessen Regierung sich verschuldet, sind zusätzlich durch die Besteuerung des Arbeitseinkommens betroffen. Hier gilt, daß die Summe aus Jugend- und Alterskonsum und der Jugendkonsum abnehmen.

Mit $\tau=\varepsilon \cdot b$ und $\bar{p}=\bar{\delta} \cdot \varepsilon /(1-\bar{\delta})$ ergeben sich die langfristigen nationalen Konsumvektoren in der integrierten Weltwirtschaft mit inländischer Staatsverschuldung wie folgt:

$$
\begin{gathered}
c_{t}^{t}(\bar{\delta}, 1-\varepsilon-\tau)=1-\varepsilon-\varepsilon \cdot b-\frac{\bar{\delta} \cdot \varepsilon}{(1-\bar{\delta})} \cdot(1+b+\bar{x})=1-\frac{\varepsilon \cdot(1+b+\bar{\delta} \cdot \bar{x})}{1-\bar{\delta}} \\
c_{t+1}^{t}(\bar{\delta}, 1-\varepsilon-\tau)=\left(\frac{\bar{\delta} \cdot \varepsilon}{1-\bar{\delta}}+\varepsilon\right) \cdot(1+b+\bar{x})=\frac{\varepsilon \cdot(1+b+\bar{x})}{1-\bar{\delta}} \\
C_{t}^{t}(\bar{\delta}, 1-\varepsilon)=1-\varepsilon-\frac{\bar{\delta} \cdot \varepsilon}{1-\bar{\delta}} \cdot(1-\bar{x})=1-\frac{\varepsilon \cdot(1-\bar{\delta} \cdot \bar{x})}{1-\bar{\delta}} \\
C_{t+1}^{t}(\bar{\delta}, 1-\varepsilon)=\left(\frac{\bar{\delta} \cdot \varepsilon}{1-\bar{\delta}}+\varepsilon\right) \cdot(1-\bar{x})=\frac{\varepsilon \cdot(1-\bar{x})}{1-\bar{\delta}}
\end{gathered}
$$


Die Gleichungen (6.11) und (6.12) geben den langfristigen Konsumvektor der Inländer an und (6.13) und (6.14) den langfristigen Konsumvektor der Ausländer. Die Gleichungen (6.13) und (6.14) stimmen hierbei mit den Gleichungen (5.21) und (5.22) überein, da das ausländische Konsumverhalten nur durch die Veränderung des Diskontfaktors beeinflußt wird (natürlich haben sich $\bar{x}$ und $\bar{\delta}$ durch die Staatsverschuldung verändert). Die nationalen Konsumvektoren sind in Darstellung 6.2 eingetragen.

\subsubsection{Die Handelsstruktur}

Für die Handelsbeziehungen zwischen den beiden Ländern ist es von entscheidender Bedeutung, in welchem der beiden Länder, Gläubiger- oder Schuldnerland, die Staatsverschuldung stattfindet. Darstellung 6.2 zeigt den Fall, in dem sich der Staat des Gläubigerlandes verschuldet. Man erkennt, daß sich die Handelsströme zwischen den beiden Ländern mit zunehmender Staatsverschuldung reduzieren. $b$ ist der langfristige ausländische Konsumplan, würde kein Außenhandel stattfinden, und $c$ der inländische. Mit steigender inländischer Staatsverschuldung dreht sich die ausländische Budgetgerade gegen den Uhrzeigersinn. $a$ (der langfristige gleichgewichtige Konsumvektor der Ausländer) und $b$ rücken hierbei immer dichter zusammen. Dies bedeutet, daß die Auslandsinvestitionen des Inlands und damit der Handelsbilanzüberschuß des Auslandes mit steigender inländischer Staatsverschuldung abnimmt. Treffen $a$ und $b$ zusammen, ist die Handelsbilanz ausgeglichen. In dieser Situation verhält sich die integrierte Weltwirtschaft allerdings wie eine Welt zweier sich in Autarkie befindender Länder, da nun keinerlei Handelsbeziehungen zwischen den Ländern existieren. Jetzt stimmen der inländische und der ausländische Autarkiezinssatz überein, und dieser ist damit auch der Zinssatz der integrierten Weltwirtschaft. Steigt die Staatsverschuldung im Inland weiter an, wird dieses zu einem Schuldnerland und das Ausland zum Gläubigerland. Dies ist deswegen der Fall, da nun der Autarkiezinssatz im Inland höher als im Ausland ist.

Im Fall, daß sich der ausländische Staat verschuldet, gilt folgendes: Je höher die Staatsverschuldung im Ausland ist, desto höher ist der ausländische Autarkie- 
zinssatz. Hier akzentuiert die Staatsverschuldung den Unterschied zwischen Inland und Ausland. Somit geht eine erhöhte Staatsverschuldung mit einer erhöhten Auslandsverschuldung einher (die Auslandsinvestitionen des Inlands nehmen mit der ausländischen Staatsverschuldung zu). Folglich erhöht sich auch der Handelsbilanzüberschuß des Auslands mit der Staatsverschuldung.

\subsubsection{Die Wohlfahrt}

Wie wirkt sich nun die inländische Staatsverschuldung auf die Wohlfahrt der Inländer und die der Ausländer aus? Durch die inländische Staatsverschuldung vergrößert sich die Budgetmenge der Ausländer (ihre Budgetgerade dreht sich bei wachsender inländischer Staatsverschuldung um den Einkommenspunkt gegen den Uhrzeigersinn). Wie man in Darstellung 6.2 erkennt, wählen die ausländischen Akteure den Konsumplan $a$ und nicht einen der Konsumpläne, den sie im langfristigen Gleichgewicht mit keiner oder einer geringeren inländischen Staatsverschuldung wählen würden (es ist kein solcher Konsumplan eingezeichnet, sie liegen aber alle innerhalb der aktuellen Budgetmenge). Somit wird $a$ gegenüber diesen Konsumplänen streng offenbart präferiert. Die Argumentation (Theorie der offenbarten Präferenz) zeigt, daß mit steigender Staatsverschuldung in einem Land die Wohlfahrt der Akteure im anderen Land zunimmt. Dieser Effekt ist dabei unabhängig davon, ob es sich um ein Schuldnerland oder ein Gläubigerland handelt. Entscheidend ist, daß sich durch die öffentliche Verschuldung in einem Land die Budgetmenge der Akteure des anderen Landes durch das Absinken des Diskontfaktors vergrößert (Verbesserung der intertemporalen Terms of Trade), was nutzenstiftendere Konsumpläne durchführbar werden läßt.

Die Wohlfahrt der Inländer wird durch die Staatsverschuldung reduziert. Dies läßt sich mit Hilfe von Roy's Identität zeigen. ${ }^{1}$ Sei $v(\bar{\delta}, w)$ die indirekte Nutzenfunktion der inländischen Akteure im langfristigen Gleichgewicht der integrierten Weltwirtschaft. Die indirekte Nutzenfunktion gibt den bei gegebenem

${ }^{1}$ Zur Royschen Identität siehe Mas-Colell et al. (1995), S. 73ff. 
Diskontfaktor und verfügbarem Einkommen maximal erreichbaren Nutzen an. Es gilt folgender Zusammenhang:

$$
c_{t+1}^{t}(\bar{\delta}, w)=-\frac{\partial v(\bar{\delta}, w) / \partial \bar{\delta}}{\partial v(\bar{\delta}, w) / \partial w} . \quad \text { (Roy's Identität) }
$$

Roy's Identität besagt, daß der negative Wert des Quotienten aus der Ableitung der indirekten Nutzenfunktion nach dem Güterpreis ( $\bar{\delta}$ ist der Gegenwartspreis des Zukunftskonsums) und der Ableitung der indirekten Nutzenfunktion nach dem verfügbaren Einkommen gleich der (Marshall'schen) Nachfragefunktion des betreffenden Gutes ist (es gilt hierbei $\partial v / \partial \bar{\delta}<0$ und $\partial v / \partial w>0$ ). ${ }^{1}$

Bedenkt man, daß $\bar{\delta}$ von $b$ determiniert wird und daß $w=1-\varepsilon-\varepsilon \cdot b$ gilt, folgt unter Verwendung von Gleichung (6.15):

$$
\begin{aligned}
\frac{d v(\bar{\delta}(b), 1-\varepsilon-\varepsilon \cdot b)}{d b} & =\frac{\partial v}{\partial \bar{\delta}} \cdot \frac{d \bar{\delta}}{d b}-\varepsilon \cdot \frac{\partial v}{\partial w}= \\
& =-\frac{\partial v}{\partial w} \cdot\left(c_{t+1}^{t}(\bar{\delta}(b), 1-\varepsilon-\varepsilon \cdot b) \cdot \frac{d \bar{\delta}}{d b}+\varepsilon\right) .
\end{aligned}
$$

Im langfristigen Gleichgewicht sind die individuellen Konsummengen der Jungen und der Alten über die Zeit konstant. Somit läßt sich Gleichung (6.10), unter Verwendung der Gleichungen (6.7)-(6.9), wie folgt schreiben:

$$
\frac{d \bar{\delta}}{d b}=\frac{\varepsilon+\varepsilon \cdot(1-\bar{\delta}) \cdot \partial c_{t+1}^{t} / \partial w}{-c_{t+1}^{t}-C_{t+1}^{t}+(1-\bar{\delta}) \cdot\left(\partial c_{t+1}^{t} / \partial \bar{\delta}+\partial C_{t+1}^{t} / \partial \bar{\delta}\right)}
$$

Aus der Slutsky-Gleichung folgt: ${ }^{2}$

$$
\partial c_{t+1}^{t} / \partial \bar{\delta}<-c_{t+1}^{t} \cdot \partial c_{t+1}^{t} / \partial w<0
$$

${ }^{1} \mathrm{Da}$ (6.15) gilt, läßt sich mit dem Enveloppen-Theorem beweisen; siehe dazu Mas-Colell et al. (1995), S.74.

${ }^{2}$ Zur Slutsky-Gleichung siehe z.B. Mas-Colell et al. (1995), S. 71 . 
(6.18) besagt, daß die Gesamtauswirkung einer Preisveränderung auf das Konsumverhalten betragsmäßig größer ist, als der isolierte Einkommenseffekt. In Verbindung mit $C_{t+1}^{t} \geq 0, \partial C_{t+1}^{t} / \partial \bar{\delta}<0, \varepsilon \in(0,1)$ und $\bar{\delta} \in(0,1)$ folgt daraus:

$$
\begin{aligned}
\varepsilon \cdot\left[-C_{t+1}^{t}+(1-\bar{\delta}) \cdot\left(\partial c_{t+1}^{t} / \partial \bar{\delta}+\partial C_{t+1}^{t} / \partial \bar{\delta}\right)\right] \\
<-c_{t+1}^{t} \cdot \varepsilon \cdot(1-\bar{\delta}) \cdot \partial c_{t+1}^{t} / \partial w<0
\end{aligned}
$$

Mit dieser Erkenntnis folgt aus Gleichung (6.17), daß $d \bar{\delta} / d b>-\varepsilon / c_{t+1}^{t}$ gilt. Somit ist in Gleichung (6.16) der Ausdruck in Klammern positiv. Da der indirekte Nutzen mit dem verfügbaren Einkommen zunimmt, sinkt der indirekte Nutzen der Akteure mit dem Verschuldungsgrad ihrer Regierung $(d v / d b<0)$. Somit reduziert die Staatsverschuldung, wie schon im Fall der geschlossenen Ökonomie, die Wohlfahrt der Akteure. Auch in der integrierten Weltwirtschaft überkompensiert der negative Effekt der Reduktion des verfügbaren Einkommens den positiven Effekt der Erhöhung der Verzinsung der Ersparnis. Dieses Ergebnis ist wieder unabhängig davon, ob das betrachtete Land ein Schuldneroder ein Gläubigerland ist.

Vergleicht man die Ergebnisse bezüglich der Wohlfahrt mit denen, die im auf zwei Länder erweiterten Diamond-Modell resultieren, so werden fundamentale Unterschiede offenbar. Wie Persson (1985) zeigt, hängen die Wohlfahrtseffekte entscheidend davon ab, ob das Land, in dem die öffentliche Verschuldung stattfindet, ein Gläubiger- oder ein Schuldnerland ist. ${ }^{1}$ Wie im vorliegenden Modell erhöht die nationale Staatsverschuldung den Zinssatz im langfristigen Gleichgewicht der integrierten Weltwirtschaft. Ein erhöhter Zinssatz geht mit einer verminderten Kapitalintensität einher, was den Kapitalertrag erhöht und den Lohnsatz reduziert. Handelt es sich bei der betrachteten Ökonomie um ein Schuldnerland, so wird ein Teil des nationalen Kapitalstocks von Ausländern gehalten. Als Folge der Erhöhung des Zinssatzes reduzieren sich somit die Konsummöglichkeiten der betreffenden Ökonomie. Handelt es sich bei der betrachteten Ökonomie allerdings um ein Gläubigerland, so vergrößert der durch die Erhöhung des Zinssatzes induzierte intertemporale Terms of Trade Effekt die Konsummöglichkeiten. Die Besteuerung des Arbeitseinkommens und die Re-

\footnotetext{
${ }^{1}$ Vgl. Persson (1985), S. 80ff.
} 
duktion des Lohns reduzieren das verfügbare Einkommen der jungen Akteure. Ist im Fall des Gläubigerlandes der positive intertemporale Terms of Trade Effekt hinreichend groß, so ist es möglich, daß er die negative Auswirkung der Reduktion des verfügbaren Arbeitseinkommens überkompensiert, so daß die Wohlfahrt der Akteure im langfristigen Gleichgewicht mit der nationalen Staatsverschuldung zunimmt. Im Fall des Schuldnerlandes reduziert sich die Wohlfahrt der Akteure, da sich hier der Terms of Trade Effekt negativ auswirkt. $^{1}$

Auch die Wohlfahrt der Akteure des anderen Landes hängt davon ab, ob es sich bei diesem um ein Schuldner- oder um ein Gläubigerland handelt. Der einzige Unterschied besteht darin, daß das verfügbare Einkommen der jungen Akteure dieses Landes nur durch die Reduktion des Lohnsatzes nicht aber durch eine Besteuerung reduziert wird. Der intertemporale Terms of Trade Effekt wirkt im Fall eines Gläubigerlandes wieder positiv und im Fall eines Schuldnerlandes wieder negativ. Im ersten Fall erhöht oder reduziert sich die Wohlfahrt der Akteure mit einer Erhöhung der Staatsverschuldung im anderen Land, im zweiten Fall reduziert sie sich. Anders als im vorliegenden Modell ist es also möglich, daß die in einem Land stattfindende Staatsverschuldung die Wohlfahrt der Akteure des anderen Landes langfristig reduziert.

Die Unterschiede zwischen den Ergebnissen des hier entwickelten Modells und den Resultaten im auf zwei Länder erweiterten Diamond-Modell resultieren daraus, daß sich im letzteren mit der Erhöhung des Zinssatzes das Verhältnis der Faktorerträge verändert und damit über die Gläubiger- bzw. Schuldnerposition eine internationale Umverteilung der Konsummöglichkeiten stattfindet. Im

${ }^{1}$ Persson (1985) betrachtet den Fall des dynamisch effizienten Gleichgewichts. Betrachtet man den Fall der Überkapitalisierung (der Zinssatz ist geringer als die Wachstumsrate der Bevölkerung), so reduziert die Staatsverschuldung den Kapitalstock in Richtung des optimalen Wertes. Damit ist es möglich, daß sich auch die Wohlfahrt der Akteure eines Schuldnerlandes mit der öffentlichen Verschuldung erhöht. Die positive Auswirkung der durch die Reduktion des Kapitalstocks resultierenden Vergrößerung der Konsummöglichkeiten muß hierfür die negativen Auswirkungen der Reduktion des verfügbaren Arbeitseinkommens und der internationalen Einkommensumverteilung überkompensieren. Zur Auswirkung der Staatsverschuldung im Zwei-Länder-Diamond-Modell siehe auch Abschnitt 2.2.2.1 und Schmid (1988), S. $101 \mathrm{ff}$. 
hier entwickelten Modell wird durch die Staatsverschuldung in einem Land das Arbeitseinkommen der jungen Akteure des anderen Landes nicht reduziert. Auf ihre Wohlfahrt wirkt nur der durch die Erhöhung des Zinssatzes erzeugte positive intertemporale Terms of Trade Effekt, welcher die Rendite ihrer Ersparnis erhöht. Damit steigt ihre Wohlfahrt.

\subsection{Die Auswirkungen eines staatlichen Budgetdefizits}

Im vorherigen Abschnitt wurden die langfristigen Auswirkungen der nationalen Staatsverschuldung analysiert. Der Diskontfaktor wird reduziert bzw. der Zinssatz erhöht, und der Preis der Vermögenstitel nimmt ab. Die Wohlfahrt der Akteure des Landes, in dem sich der Staat verschuldet, wird reduziert und die Wohlfahrt der Akteure des anderen Landes wird erhöht. In diesem Abschnitt soll nun untersucht werden, was die kurzfristigen Auswirkungen eines nationalen staatlichen Budgetdefizits sind, was also auf dem Weg hin zum langfristigen Gleichgewicht mit nationaler Staatsverschuldung passiert. Es wird angenommen, daß sich die integrierte Weltwirtschaft bis Periode 0 in einem langfristigen Gleichgewicht ohne Staatsverschuldung befindet. In Periode 1 läßt die inländische Regierung dem inländischen Akteur 1 einen von den Akteuren nicht erwarteten Transfer $\left(\tau_{1}<0\right)$ zukommen. Dies erfordert gemäß der staatlichen Budgetrestriktion (4.1) eine Emission von $b=-\tau_{1} / \bar{p}_{1}$ Anleihen. Der Bestand an Staatsanleihen wird in den folgenden Perioden konstant gehalten, was in den folgenden Perioden eine Besteuerung des inländischen Arbeitseinkommens notwendig macht (Zinsendienst). Die Steuer beträgt hierbei $\tau_{t}=\varepsilon \cdot b, t=2,3, \ldots$. Da der determinierte (reguläre) Fall betrachtet wird, dauert die Übergangsphase zum neuen langfristigen Gleichgewicht nur eine Periode an. Schon in Periode 2 befindet sich die Weltwirtschaft in ihrem neuen langfristigen Gleichgewicht. 
Die Emission der inländischen Staatsanleihen erhöht die Anzahl der sich im Umlauf befindenden Vermögenstitel von 2 auf $2+b .{ }^{1}$ Die Gleichgewichtsbedingung des Weltkapitalmarktes der Periode 1 lautet somit:

$$
\bar{s}^{1}\left(\bar{\delta}_{2}, 1-\varepsilon-\varepsilon \cdot b, 1-\varepsilon\right)=\underbrace{\bar{p}_{1} \cdot 2}_{\begin{array}{c}
\text { Angebot } \\
\text { Generation } 0
\end{array}}+\underbrace{\bar{p}_{1} \cdot b}_{\begin{array}{c}
\text { Angebot } \\
\text { inlind. Staat }
\end{array}}=\bar{p}_{1} \cdot(2+b) .
$$

Die aggregierte Ersparnis der Generation 1 muß gleich dem Kurswert der ihr angebotenen Vermögenstitel sein. Die Weltgütermarkträumung in Periode 2 erfordert:

$$
\bar{c}_{2}^{2}\left(\bar{\delta}_{3}, 1-\varepsilon-\varepsilon \cdot b, 1-\varepsilon\right)+\bar{c}_{2}^{1}\left(\bar{\delta}_{2}, 1-\varepsilon-\tau_{1}, 1-\varepsilon\right)=2 .
$$

Die Summe aus Weltjugendkonsum der Generation 2 und Weltalterskonsum der Generation 1 muß gleich der Weltgüterproduktion sein.

\subsubsection{Die Entwicklung der Zinssätze}

Da sich die Weltwirtschaft bereits in Periode 2 in ihrem neuen langfristigen Gleichgewicht befindet, muß der aggregierte Konsum der Alten dieser Periode gleich dem aggregierten Konsum der Alten der folgenden Perioden sein $\left(\bar{c}_{2}^{1}\right.$ $\left.=\bar{c}_{t+1}^{t}, t=2,3,4 \ldots\right)$. Dies kann nur dann der Fall sein, wenn die Verzinsung der Ersparnis der Generation 1 geringer ist als die Verzinsung der Ersparnis der folgenden Perioden. Es gilt also $\bar{r}_{2}<\bar{r}_{3}$. Das Argument ist das gleiche wie schon im Fall der geschlossenen Ökonomie: Das Arbeitseinkommen der Ausländer wird von der inländischen Fiskalpolitik nicht berührt. Das (verfügbare Einkommen des inländischen Akteurs 1 ist, wie im Fall der geschlossenen Ökonomie, höher als das der inländischen Akteure der folgenden Generationen $\left(1-\varepsilon-\tau_{1}\right.$

\footnotetext{
${ }^{1}$ Wie man aus Kapitel 5 weiß, befinden sich bis Periode 1 tatsächlich 4 Vermögenstitel in Umlauf. Dies sind die zwei Anteilscheine an den nationalen Produktionstechniken und die zweiperiodigen Schuldscheine, die von den jungen Ausländern emittiert werden. Wie schon darauf hingewiesen wurde, heben sich die Schuldscheine makroökonomisch auf. In jeder Periode kommt auf einen emittierten Schuldschein ein eingelöster Schuldschein.
} 
$>1-\varepsilon-\varepsilon \cdot b$ ). Somit ist das aggregierte verfügbare Einkommen der Generation 1 höher als das aggregierte verfügbare Einkommen der folgenden Generationen. Ein erhöhtes Einkommen erhöht den Konsum in der Jugend und im Alter (Normalgut-Annahme). Da $\partial c_{t+1}^{t} / \partial \bar{\delta}_{t+1}<0$ und $\partial C_{t+1}^{t} / \partial \bar{\delta}_{t+1}<0$ gilt (unabhängig davon, ob die Güter Bruttosubstitute sind oder nicht), muß $\bar{\delta}_{2}>\bar{\delta}_{3}$ gelten.

Dies zeigt aber auch, daß zwar der aggregierte Alterskonsum der Generation 1 gleich dem Weltalterskonsum der folgenden Perioden ist, dies aber nicht für die nationalen Konsumpläne zutrifft. Der ausländische Akteur 1 wird mit einem höheren Diskontfaktor konfrontiert, als die Ausländer der folgenden Generationen. Somit ist sein Alterskonsum geringer als der Alterskonsum der Ausländer im neuen langfristigen Gleichgewicht der integrierten Weltwirtschaft. Der Alterskonsum des Inländers 1 ist entsprechend höher als der Alterskonsum der Inländer der folgenden Generationen, da der aggregierte Alterskonsum der Generation 1 mit dem aggregierten Alterskonsum der folgenden Generationen übereinstimmen muß.

Wie schon im Fall der geschlossenen Ökonomie kann das Modell keine Vorhersage darüber machen, ob der kurzfristige Zinssatz eine Periode nach Auftreten des staatlichen Budgetdefizits unter seinem alten langfristigen Niveau verbleibt oder über dieses steigt. Die Entwicklung des kurzfristigen Zinssatzes hängt vom Verlauf des Einkommensexpansionspfades der inländischen Akteure ab. Der Umstand, daß $\bar{r}_{2}<\bar{r}_{0}<\bar{r}_{3}$ oder $\bar{r}_{0}<\bar{r}_{2}<\bar{r}_{3}$ gelten kann, läßt sich graphisch veranschaulichen.

Darstellung 6.3 zeigt den inländischen Konsumplan (mit $A$ bezeichnet) im alten langfristigen, bis Periode 0 realisierten Gleichgewicht der integrierten Weltwirtschaft und den mit ihm einhergehenden Diskontfaktor $\bar{\delta}_{0}$. Die emittierte Anleihemenge $b$ wird durch die Erfordernis $b=-\tau_{1} / \bar{p}_{1}$ determiniert. Sei $b$ auf diesen Wert fixiert und $p_{1}$ derart gewählt, daß $\bar{\delta}_{2}=\bar{\delta}_{0}$ gilt. Dieser Preis werde mit $\widetilde{p}_{1}$ bezeichnet $\left(\widetilde{p}_{1}=\bar{\delta}_{0} \cdot\left(\bar{p}_{2}+\varepsilon\right)\right)$ Bei $\widetilde{p}_{1}$ verläuft die unterbrochen eingezeichnete Budgetgerade des inländischen Akteurs 1 parallel zur Budgetgeraden der inländischen Akteure im alten langfristigen Gleichgewicht. Die vertikale gestrichelte Gerade gibt die Konsummenge des alten Inländers 1 an, die gewährleistet, daß der aggregierte Alterskonsum der Generation 1, bei gegebenem Dis- 
kontfaktor $\bar{\delta}_{2}\left(=\bar{\delta}_{0}\right)$, dem Weltalterskonsum im neuen langfristigen Gleichgewicht entspricht. Da die Höhe von $\bar{\delta}_{2}$ den Alterskonsum des Ausländers 1 beeinflußt, verändert sich mit ihr auch der für ein Gleichgewicht notwendige Alterskonsum des Inländers 1 . Mit steigendem $\bar{\delta}_{2}$ reduziert sich der Alterskonsum des Ausländers $1\left(\partial C_{2}^{1} / \partial \bar{\delta}_{2}<0\right)$. Mit sinkendem $\bar{\delta}_{2}$ erhöht sich sein Alterskonsum. Entsprechend erhöht sich der für ein Gleichgewicht notwendige Alterskonsum des Inländers 1 im ersten Fall (die vertikale gestrichelte wandert nach rechts) und reduziert sich im zweiten Fall (die vertikale gestrichelte Linie wandert nach links).

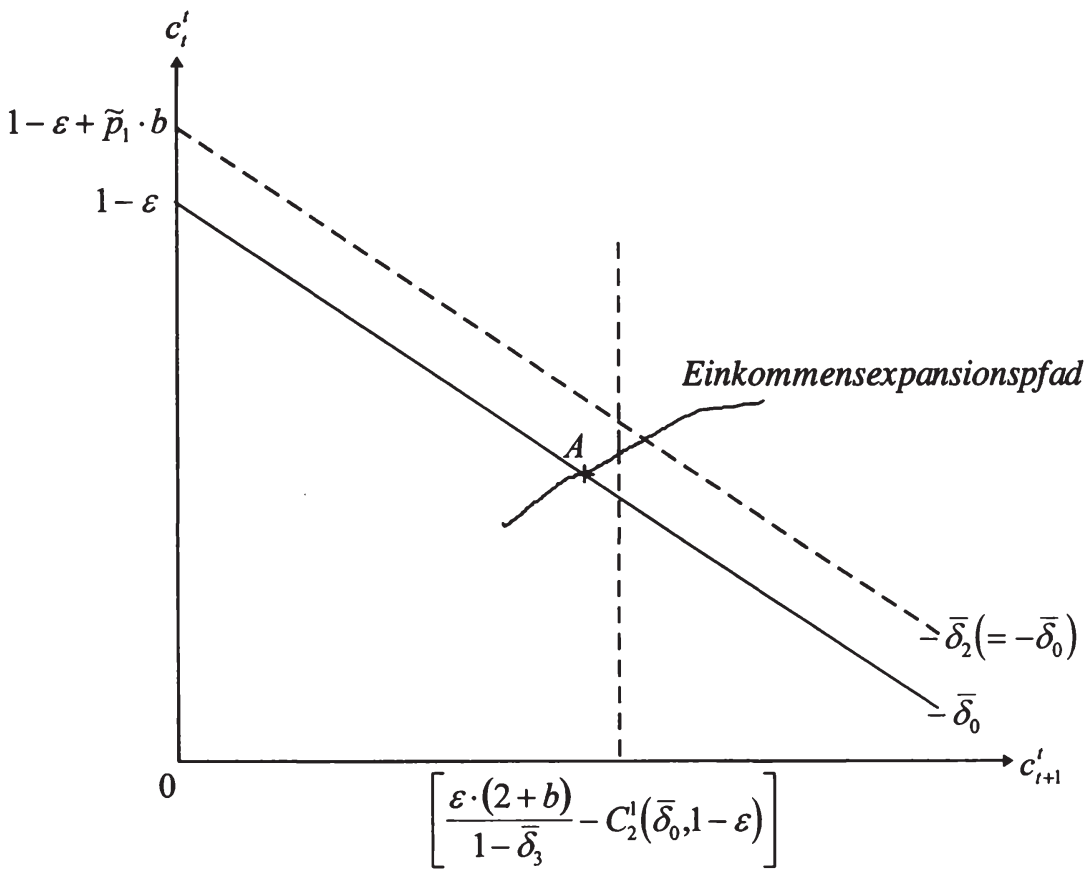

Darst. 6.3: Kurzfristiger Zinseffekt 
Die Lage des optimalen Konsumprogramms von Inländer 1, auf der unterbrochen gezeichneten Budgetgeraden, wird durch den Verlauf seines Einkommensexpansionspfades bestimmt. Darstellung (6.3) zeigt den Fall, daß dieses Programm rechts von der gestrichelten vertikalen Geraden liegt. Der alte Inländer 1 möchte bei $\bar{\delta}_{2}\left(=\bar{\delta}_{0}\right)$ somit mehr konsumieren, als das Gleichgewicht der Weltwirtschaft zuläßt. Damit Inländer $1 \mathrm{im}$ Alter weniger konsumiert, ist es notwendig, daß $\bar{\delta}_{2}$ steigt (wegen $\bar{\delta}_{2}=\widetilde{p}_{1} /\left(\varepsilon+\bar{p}_{2}\right)$ ist dies äquivalent dazu, da $\bar{p}_{1}$ über $\tilde{p}_{1}$ steigt, da $\bar{p}_{2}$ auf seinen neuen langfristigen Wert determiniert ist) bzw. die Verzinsung fällt, da $\partial c_{2}^{1} / \partial \bar{\delta}_{2}<0$ gilt. Bei steigendem $\bar{\delta}_{2}$ bewegen sich der gleichgewichtige und der vom Akteur 1 gewünschte Alterskonsum aufeinander zu. Somit ist der kurzfristige Zinssatz eine Periode nach Auftreten des inländischen staatlichen Budgetdefizits niedriger als der Zinssatz im alten langfristigen Gleichgewicht (es gilt $\bar{r}_{2}<\bar{r}_{0}<\bar{r}_{3} \Leftrightarrow \bar{\delta}_{2}>\bar{\delta}_{0}>\bar{\delta}_{3}$ ). Ganz anders verhält es sich, wenn der Einkommensexpansionspfad derart verläuft, daß der Konsumplan von Inländer 1 bei $\widetilde{p}_{1}$ links von der gestrichelten vertikalen Linie liegt. In diesem Fall konsumiert der alte Inländer $1 \mathrm{zu}$ wenig. Jetzt muß $\bar{\delta}_{2}$ sinken bzw. die Verzinsung steigen. In diesem Fall gilt $\bar{r}_{0}<\bar{r}_{2}<\bar{r}_{3} \Leftrightarrow \bar{\delta}_{0}>\bar{\delta}_{2}>\bar{\delta}_{3}$.

Vergleicht man die in Darstellung 6.3 geführte Diskussion mit der aus Darstellung 4.2, so erkennt man, daß der durch ein staatliches Budgetdefizit induzierte Zinseffekt durch die weltwirtschaftliche Integration abgeschwächt wird. Ein staatliches Budgetdefizit bewirkt, über die Veränderung des Zinssatzes, eine Veränderung im Konsumverhalten der Akteure des anderen Landes. Der Anpassungsbedarf im Konsumverhalten der Akteure des Landes, dessen Staat sich verschuldet, reduziert sich damit.

Der Effekt des Budgetdefizits auf den langfristigen Zinssatz bzw. den Preis der Vermögenstitel ist wie folgt (man erinnere sich, da $\bar{r}_{t+1}^{l}=\varepsilon / p_{t}$ gilt): $\mathrm{Da} \beta$ $\bar{p}_{0}>\bar{p}_{2}$ bzw. $\bar{r}_{1}^{l}<\bar{r}_{3}^{l}$ gilt ist aus der Analyse der langfristigen Auswirkungen der Staatsverschuldung bekannt. Des weiteren ist der Preis der Vermögenstitel in der Übergangsphase zum neuen langfristigen Gleichgewicht höher als in diesem bzw. der langfristige Zinssatz geringer. Es gilt also $\bar{p}_{1}>\bar{p}_{2}$ bzw. $\bar{r}_{2}^{l}<\bar{r}_{3}^{l}$. Dies folgt aus Beziehung (5.5) und der Tatsache, daß $\bar{\delta}_{2}>\bar{\delta}_{3}$ gilt, denn $\bar{\delta}_{2}$ $=\bar{p}_{1} /\left(\varepsilon+\bar{p}_{2}\right)$ und $\bar{\delta}_{3}=\bar{p}_{2} /\left(\varepsilon+\bar{p}_{2}\right)$. 
Wie schon im Fall der geschlossenen Ökonomie, ist eine Aussage über die Entwicklung des Preises der Vermögenstitel im Verhältnis zu ihrem Preis im alten langfristigen Gleichgewicht, ohne Annahme an die Beziehung zwischen dem Konsum in der Jugend und dem Konsum im Alter, nicht möglich. Nimmt man an, daß der Konsum in der Jugend und der Konsum im Alter in beiden Ländern Bruttosubstitute sind, also $\partial c_{t}^{t} / \partial \bar{\delta}_{t+1}>0$ und $\partial C_{t}^{t} / \partial \bar{\delta}_{t+1}>0$ gilt, läßt sich, wie schon im Fall der geschlossenen Ökonomie, durch einen Widerspruchsbeweis leicht zeigen, daß der Preis der Vermögenstitel in der Übergangsphase hin zum neuen langfristigen Gleichgewicht unter den Preis im alten langfristigen Gleichgewicht sinkt, also $\bar{p}_{0}>\bar{p}_{1}$ bzw. $\bar{r}_{1}^{l}<\bar{r}_{2}^{l}$ gilt: $^{1}$

Sei angenommen, daß $\bar{p}_{0} \leq \bar{p}_{1}$. Damit gilt $\bar{c}_{1}^{0}\left(=2 \cdot\left(\varepsilon+\bar{p}_{1}\right)\right) \geq \bar{c}_{0}^{-1}\left(=2 \cdot\left(\varepsilon+\bar{p}_{0}\right)\right)$. Gemäß der Gleichgewichtsbedingung für den Weltkapitalmarkt (6.2) ist dies äquivalent zu $\bar{c}_{0}^{0}\left(\bar{\delta}_{0}, 1-\varepsilon, 1-\varepsilon\right) \geq \bar{c}_{1}^{1}\left(\bar{\delta}_{2}, 1-\varepsilon-\tau_{1}, 1-\varepsilon\right)$ Durch den dem inländischen Akteur 1 zukommenden Transfer ist das aggregierte Einkommen der Generation 1 höher als das der Generation 0. Ein erhöhtes aggregiertes Einkommen wirkt aufgrund der Normalgut-Annahme in Richtung einer Erhöhung des Weltkonsums in der Jugend und im Alter. Eine notwendige Bedingung für $\bar{c}_{0}^{0} \geq \bar{c}_{1}^{1}$ ist somit, daß der von Generation 0 erwartete Diskontfaktor höher ist, als der von Generation 1 erwartete. $\bar{c}_{0}^{0} \geq \bar{c}_{1}^{1}$ impliziert also $\bar{\delta}_{0}>\bar{\delta}_{2}$. Dies stellt jedoch einen Widerspruch zu $\bar{p}_{0}>\bar{p}_{2}$ und der Annahme $\bar{p}_{0} \leq \bar{p}_{1}$ dar, denn $\bar{\delta}_{2}$ $=\bar{p}_{1} /\left(\bar{p}_{2}+\varepsilon\right) \geq \bar{p}_{0} /\left(\bar{p}_{2}+\varepsilon\right)>\bar{p}_{0} /\left(\bar{p}_{0}+\varepsilon\right)=\bar{\delta}_{0}$.

Damit zeigt sich, daß unter der von nun an geltenden Annahme, daß die Güter in beiden Ländern Bruttosubstitute sind, der Preis der Vermögenstitel auf dem Weg hin zum neuen langfristigen Gleichgewicht monoton abnimmt bzw. der langfristige Zinssatz monoton steigt. Mit der Erkenntnis, daß $\bar{p}_{-1}=\bar{p}_{0}>\bar{p}_{1}>\bar{p}_{2}$ $=\bar{p}_{3} \Leftrightarrow \bar{r}_{0}^{l}=\bar{r}_{1}^{l}<\bar{r}_{2}^{l}<\bar{r}_{3}^{l}=\bar{r}_{4}^{l}$ gilt, läßt sich eine vollständige Beschreibung der durch das Budgetdefizit induzierten Zinsdynamik durchführen. Hierfür formt man die Beziehung (5.5) entsprechend der Zinsarbitragebedingung (3.2) um und erhält damit:

${ }^{1}$ Die Eigenschaft der Güter Bruttosubstitute zu sein ist also eine hinreichende Bedingung dafür, eine eindeutige Aussage über die Entwicklung des Preises der Vermögenstitel bzw. des langfristigen Zinssatzes treffen zu können. Die Annahme ist stärker als die der Determiniertheit; vgl. Abschnitt 3.2. 


$$
\begin{aligned}
& \bar{r}_{0}=\frac{\varepsilon}{\bar{p}_{-1}}+\underbrace{\frac{\bar{p}_{0}-\bar{p}_{-1}}{\bar{p}_{-1}}}_{=0}=\bar{r}_{0}^{l}-\underbrace{\frac{\bar{r}_{1}^{l}-\bar{r}_{0}^{l}}{\bar{r}_{1}^{l}}}_{=0} \Leftrightarrow \bar{r}_{0}=\bar{r}_{0}^{l}, \\
& \bar{r}_{1}=\frac{\varepsilon}{\bar{p}_{0}}+\underbrace{\frac{\bar{p}_{1}-\bar{p}_{0}}{\bar{p}_{0}}}_{<0}=\bar{r}_{1}^{l}-\underbrace{\frac{\bar{r}_{2}^{l}-\bar{r}_{1}^{l}}{\bar{r}_{2}^{l}}}_{>0} \Leftrightarrow \bar{r}_{1}<\bar{r}_{1}^{l}, \\
& \bar{r}_{2}=\frac{\varepsilon}{\bar{p}_{1}}+\underbrace{\frac{\bar{p}_{2}-\bar{p}_{1}}{\bar{p}_{1}}}_{<0}=\bar{r}_{2}^{l}-\underbrace{\frac{\bar{r}_{3}^{l}-\bar{r}_{2}^{l}}{\bar{r}_{3}^{l}}}_{>0} \Leftrightarrow \bar{r}_{2}<\bar{r}_{2}^{l}, \\
& \bar{r}_{3}=\frac{\varepsilon}{\bar{p}_{2}}+\underbrace{\frac{\bar{p}_{3}-\bar{p}_{2}}{\bar{p}_{2}}}_{=0}=\bar{r}_{3}^{l}-\underbrace{\frac{\bar{r}_{4}^{l}-\bar{r}_{3}^{l}}{\bar{r}_{4}^{l}}}_{=0} \Leftrightarrow \bar{r}_{3}=\bar{r}_{3}^{l} .
\end{aligned}
$$

Bis Periode 0 sind der kurzfristige und der langfristige Zinssatz auf ihrem alten langfristigen Niveau, wobei die Zinsstruktur flach ist. In Periode 1 erzeugt die nicht antizipierte Emission der inländischen Staatsanleihen ein Absinken des Preises der Vermögenstitel. Dies erzeugt ein Kapitalverlust, den die Generation 0 vollständig tragen muß (die von ihr gehaltenen Vermögenstitel verlieren an Kurswert). Somit sinkt der kurzfristige Zinssatz zwischen Periode 0 und Periode 1 auf $\bar{r}_{1}\left(<\bar{r}_{0}\right)$, wobei die Zinsstruktur in Periode 1 normal ist. Da das Modell keine Aussage dazu machen kann, ob der kurzfristige Zinssatz in Periode 2 geringer oder höher ist als der kurzfristige Zinssatz im alten langfristigen Gleichgewicht, sind zwei Fälle zu unterscheiden: Im Fall, daß $\bar{r}_{2}<\bar{r}_{0}$ gilt, ist nicht klar, ob der kurzfristige Zinssatz zwischen Periode 1 und Periode 2 sinkt oder steigt. Dies folgt aus den Gleichungen (6.23) und (6.24). Zwar ist die Ertragskomponente der Rendite in Periode 2 größer als in Periode $1\left(\bar{p}_{1}<\bar{p}_{0} \Leftrightarrow \varepsilon / \bar{p}_{1}\right.$ $>\varepsilon / \bar{p}_{0}$ ), das Modell macht aber keine Aussagen über die Ausprägung der Kapitalverlustkomponente in den beiden Perioden. Der zweite Fall ist der, daß $\bar{r}_{2}>\bar{r}_{0}$ gilt. In diesem Fall läßt sich eine Aussage darüber machen, wie sich der kurzfristige Zinssatz zwischen der Periode 1 und der Periode 2 entwickelt. Da $\bar{r}_{1}<\bar{r}_{0}$ gilt, muß der kurzfristige Zinssatz zwischen Periode 1 und Periode 2 
Egal, welcher Fall vorliegt, ist die Zinsstruktur in Periode 2 normal. Dies muß deshalb der Fall sein, da die Vermögenstitel zwischen Periode 1 und Periode 2 an Kurswert verlieren. In Periode 3 stimmen der kurzfristige und der langfristige Zinssatz wieder überein. Der Kurswert der Vermögenstitel bleibt ab Periode 2 konstant, daß sich die Weltwirtschaft ab dieser Periode in ihrem neuen langfristigen Gleichgewicht befindet. Der kurzfristige und der langfristige Zinssatz steigen zwischen Periode 2 und Periode 3, wobei die Veränderung des kurzfristigen Zinssatzes ausgeprägter ist als die des langfristigen Zinssatzes (die Zinsstruktur war in Periode 2 noch normal, in Periode 3 ist sie flach). Zum einen erhöht sich der kurzfristige Zinssatz in Periode 3 durch seine vergrößerte Ertragskomponente $\left(\bar{p}_{2}<\bar{p}_{1} \Leftrightarrow \varepsilon / \bar{p}_{2}>\varepsilon / \bar{p}_{1}\right)$, zum anderen findet in Periode 3 kein Kapitalverlust statt. Die möglichen Zinspfade lassen sich wieder graphisch veranschaulichen, was in Darstellung 6.4 geschieht.

Vergleicht man die Resultate mit denen aus Abschnitt 4.2.1, erkennt man, daß sich die durch ein staatliches Budgetdefizit induzierte Zinsdynamik, die in der integrierten Weltwirtschaft resultiert, in qualitativer Hinsicht nicht von der in der geschlossenen Ökonomie unterscheidet. Dies war zu erwarten. Es gilt wie im Fall der geschlossenen Ökonomie $\left.\bar{p}_{0}>\bar{p}_{1}\right\rangle \bar{p}_{2} \Leftrightarrow \bar{r}_{1}^{l}<\bar{r}_{2}^{l}<\bar{r}_{3}^{l}$. Da sich auch die mögliche Entwicklung des kurzfristigen Zinssatzes nicht von der in der geschlossenen Ökonomie unterscheidet (es gilt $\bar{r}_{2}<\bar{r}_{0}<\bar{r}_{3}$ oder $\bar{r}_{0}<\bar{r}_{2}<\bar{r}_{3}$ ), stimmen die Gleichungen (6.22)-(6.25) mit den Gleichungen (4.9)-(4.12) überein. In (6.22)-(6.25) sind nur alle Werte mit einem Querstrich versehen, da sie auf den Weltmärkten determiniert werden.

Die qualitative Übereinstimmung der Ergebnisse ist nicht verwunderlich, ist doch eine integrierte Weltwirtschaft nichts anderes als eine Welt einer einzelnen geschlossenen Ökonomie. Betrachtet man die integrierte Weltwirtschaft als solche, so sieht man sich einer geschlossenen Ökonomie gegenüber, die von zwei sich durch die Zeitpräferenz unterscheidenden Gruppen bevölkert wird, deren Generationen jeweils durch einen einzelnen repräsentativen Akteur beschrieben werden. Dies zeigt, daß die eruierte Zinsdynamik kein Artefakt ist, welches aus der Annahme eines einzelnen repräsentativen Akteurs resultiert, der die jeweilige Generation verkörpern soll. 
Zinsniveau

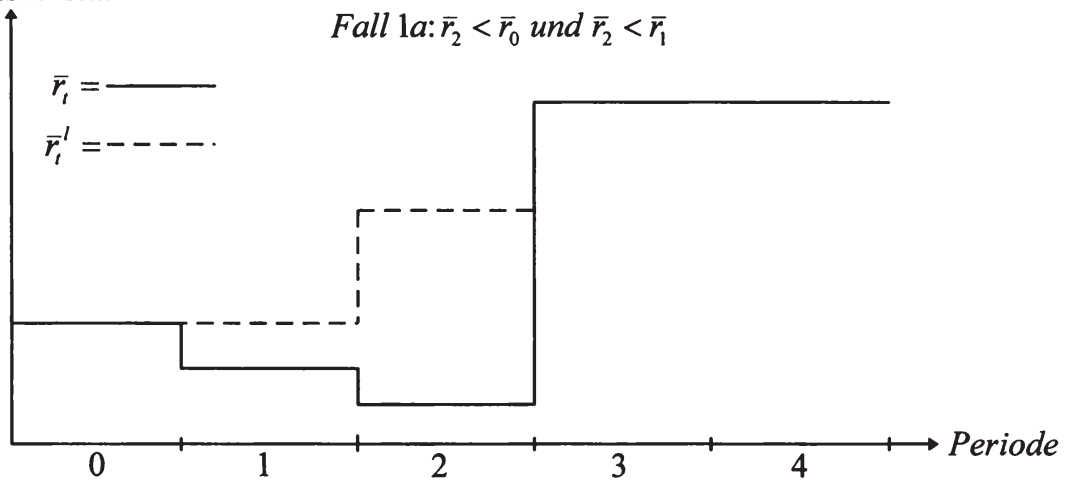

Zinsniveau

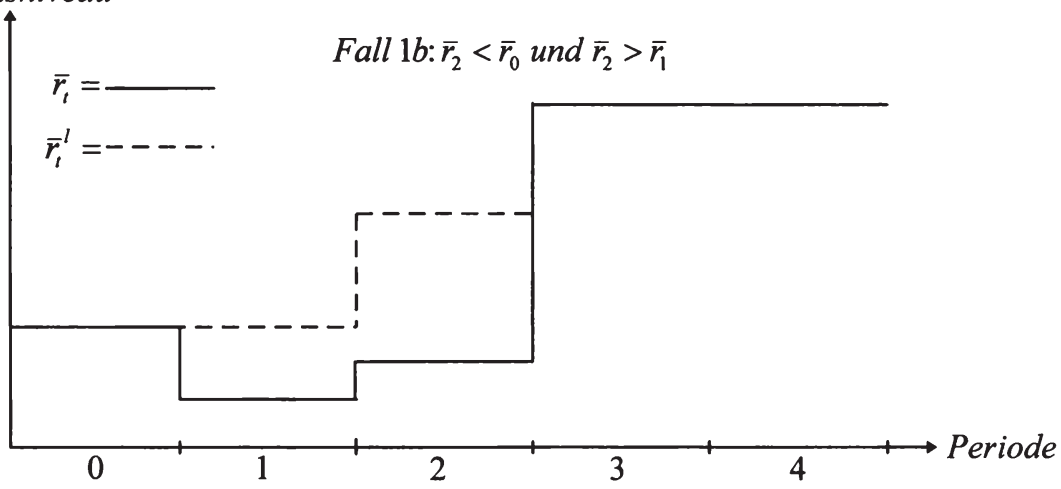

Zinsniveau

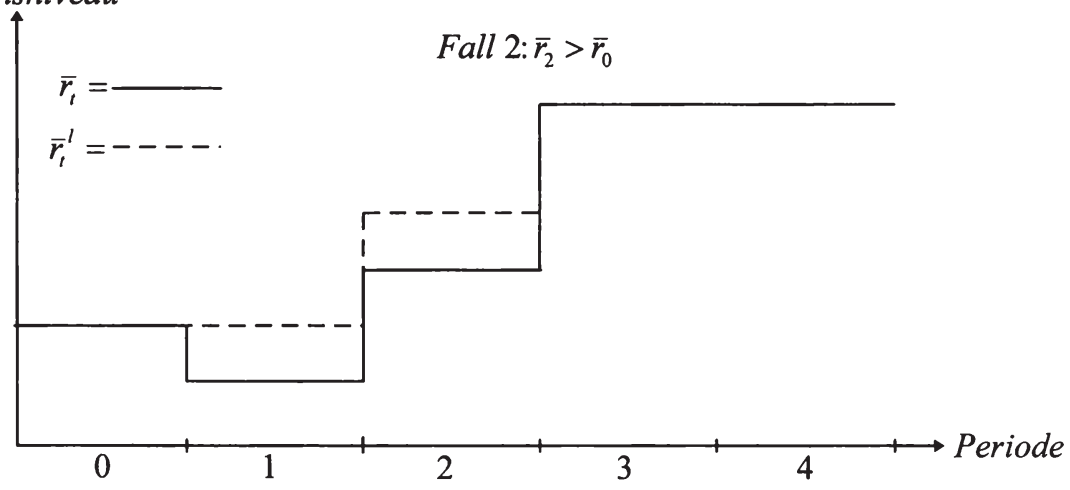

Darst. 6.4: Mögliche Zinsdynamik 


\subsubsection{Die Auswirkung auf die Wohlfahrt}

Wie wirkt sich das Budgetdefizit des inländischen Staates auf die Wohlfahrt der von ihm betroffenen Generationen aus? Generation 0 wird in Periode 1 von dem unerwarteten Budgetdefizit überrascht. $c_{0}^{0}$ und $C_{0}^{0}$ bleiben von dem Budgetdefizit unberührt, da es von den Akteuren nicht antizipiert wird. In Periode 1 muß Generation 0 allerdings ein Kapitalverlust hinnehmen, da der Preis der von ihr gehaltenen Vermögenstitel durch das Budgetdefizit reduziert wird. Somit sinkt $c_{1}^{0}$ und $C_{1}^{0}$ unter den Wert des alten langfristigen Gleichgewichts und die Wohlfahrt der Generation 0 wird in beiden Ländern reduziert.

Will man den durch das inländische staatliche Budgetdefizit induzierten Wohlfahrtseffekt auf die Generation 1 eruieren, muß man die Veränderung des Konsumverhaltens des inländischen und des ausländischen Akteurs 1 separat analysieren. Es wird nun gezeigt, daß sich sowohl der Jugendkonsum als auch der Alterskonsum des inländischen Akteurs 1 durch das Budgetdefizit gegenüber dem inländischen Konsumplan im alten langfristigen Gleichgewicht erhöht: Es gilt $\bar{c}_{0}^{-1}+\bar{c}_{0}^{0}=\bar{c}_{1}^{0}+\bar{c}_{1}^{1}=2$ (Gütermarkträumung). Aufgrund des Kapitalverlustes, den die Generation 0 hinnehmen muß, gilt $\bar{c}_{0}^{-1}>\bar{c}_{1}^{0}$. Aus der Verbindung beider Erkenntnisse folgt $\bar{c}_{0}^{-1}-\bar{c}_{1}^{0}=\bar{c}_{1}^{1}-\bar{c}_{0}^{0}=\left(c_{1}^{1}-c_{0}^{0}\right)+\left(C_{1}^{1}-C_{0}^{0}\right)>0$. Gilt $C_{1}^{1} \leq C_{0}^{0}$, impliziert dies $c_{1}^{1}>c_{0}^{0}$. Gilt $C_{1}^{1}>C_{0}^{0}$, ist dies äquivalent zu $\bar{\delta}_{2}>\bar{\delta}_{0}$ (Bruttosubstitute). ${ }^{1} \bar{\delta}_{2}>\bar{\delta}_{0}$ impliziert, daß $c_{1}^{1}>c_{0}^{0}$ gilt. Das verfügbare Einkommen des inländischen Akteurs 1 ist durch dem ihm zukommenden Transfer höher als das des inländischen Akteurs 0. Dies verstärkt aufgrund der Normalgut-Eigenschaft den durch die Erhöhung des Diskontfaktors erzeugten positiven Effekt auf den Jugendkonsum zusätzlich. Somit erhöht sich der Jugendkonsum des inländischen Akteurs 1 gegenüber dem Jugendkonsum der inländischen Akteure im alten langfristigen Gleichgewicht in jedem Fall.

Aus der Analyse der langfristigen Effekte der nationalen Staatsverschuldung weiß man, daß diese eine Erhöhung des aggregierten Konsums der Alten be-

\footnotetext{
${ }^{1}$ Man beachte, daß Generation 0 ihre Sparentscheidung aufgrund des von ihr erwarteten Diskontfaktors trifft. Da sie das Budgetdefizit der Periode 1 nicht antizipiert, rechnet sie für diese Periode mit einem Diskontfaktor, der dem der Periode 0 entspricht (in Periode 0 befindet sich die Weltwirtschaft noch im alten langfristigen Gleichgewicht).
} 
wirkt. Es gilt also $c_{2}^{1}+C_{2}^{1}>c_{0}^{-1}+C_{0}^{-1}>0$. Dies ist äquivalent zu $\left(c_{2}^{1}-c_{0}^{-1}\right)$ $+\left(C_{2}^{1}-C_{0}^{-1}\right)>0$. Wenn $C_{2}^{1} \leq C_{0}^{-1}$ gilt, folgt daraus $c_{2}^{1}>c_{0}^{-1}$. Gilt $C_{2}^{1}>C_{0}^{-1}$, ist dies äquivalent zu $\bar{\delta}_{0}>\bar{\delta}_{2}$ (der Alterskonsum nimmt mit steigendem Diskontfaktor ab). Aus $\bar{\delta}_{0}>\bar{\delta}_{2}$ und aus dem durch den Transfer erhöhten verfügbaren Einkommen des inländischen Akteurs 1 folgt, daß auch in diesem Fall $c_{2}^{1}>c_{0}^{-1}$ gilt. Somit erhöht sich auch der Alterskonsum des inländischen Akteurs 1 gegenüber dem Alterskonsum der inländischen Akteure im alten langfristigen Gleichgewicht in jedem Fall. Dies bedeutet, daß die Wohlfahrt des inländischen Akteurs 1 durch das Budgetdefizit des inländischen Staates erhöht wird. Dieses Ergebnis war zu erwarten, da ihm ein Transfer zukommt.

Das Konsumverhalten des ausländischen Akteurs 1 im Vergleich zum Konsumverhalten der ausländischen Akteure im alten langfristigen Gleichgewicht hängt davon ab, ob der Diskontfaktor eine Periode nach Auftreten des inländischen staatlichen Budgetdefizits über seinem alten langfristigen Niveau verharrt, oder unter dieses sinkt, ob also $\bar{\delta}_{0}<\bar{\delta}_{2}$ oder $\bar{\delta}_{0}>\bar{\delta}_{2}$ gilt. Im Fall, daß $\bar{\delta}_{0}<\bar{\delta}_{2}$ gilt, reduziert sich der Alterskonsum und erhöht sich wegen der BruttosubstitutEigenschaft der Güter der Jugendkonsum. Gilt $\bar{\delta}_{0}>\bar{\delta}_{2}$, resultiert eine Erhöhung des Alterskonsums und eine Reduktion des Jugendkonsums.

Im Fall, daß $\bar{\delta}_{0}<\bar{\delta}_{2}$ gilt, verkleinert sich die Budgetmenge des ausländischen Akteurs 1 gegenüber der Budgetmenge der ausländischen Akteure im alten langfristigen Gleichgewicht. Der Konsumplan, den die ausländischen Akteure im alten langfristigen Gleichgewicht wählen, ist für den ausländischen Akteur 1 nicht mehr durchführbar. Sein Konsumplan liegt innerhalb der Budgetmenge der ausländischen Akteure im alten langfristigen Gleichgewicht. Somit wird der Konsumplan des alten langfristigen Gleichgewichts gegenüber dem Konsumplan des Akteurs 1 von den ausländischen Akteuren streng offenbart präferiert. Dies impliziert, daß der ausländische Konsumplan im alten langfristigen Gleichgewicht echt nutzenstiftender ist als der des ausländischen Akteurs 1. Im Fall, daß $\bar{\delta}_{0}<\bar{\delta}_{2}$ gilt, reduziert das Budgetdefizit des inländischen Staates somit die Wohlfahrt des ausländischen Akteurs 1.

Gilt $\bar{\delta}_{0}>\bar{\delta}_{2}$, vergrößert das Budgetdefizit des inländischen Staates die Budgetmenge des ausländischen Akteurs 1 gegenüber der Budgetmenge der ausländi- 
schen Akteure im alten langfristigen Gleichgewicht. Der ausländische Konsumplan im alten langfristigen Gleichgewicht liegt innerhalb der Budgetmenge des ausländischen Akteurs 1. Der von ihm gewählte Konsumplan wird von ihm gegenüber dem ausländischen Konsumplan des alten langfristigen Gleichgewichts streng offenbart präferiert. Somit ist der Konsumplan des ausländischen Akteurs 1 echt nutzenstiftender als der Konsumplan der ausländischen Akteure im alten langfristigen Gleichgewicht. Im Fall, daß $\bar{\delta}_{0}>\bar{\delta}_{2}$ gilt, erhöht das Budgetdefizit des ausländischen Staates somit die Wohlfahrt des ausländischen Akteurs 1.

Die Auswirkungen auf die folgenden Generationen wurden schon bei der Analyse der langfristigen Auswirkungen der Staatsverschuldung gezeigt. Die Wohlfahrt der ausländischen Akteure der folgenden Generationen nimmt zu, die der inländischen Akteure der folgenden Generationen nimmt ab.

Vergleicht man die Ergebnisse mit denen im Zwei-Länder-Diamond-Modell, so werden wieder fundamentale Unterschiede offenbar. ${ }^{1}$ Die Generation, die in der Periode eines staatlichen Budgetdefizits alt ist, bleibt wie im Fall der geschlossenen Ökonomie von diesem unberührt. Der Grund ist wieder der, daß es innerhalb des Diamond-Modells keine Kapitalverluste bzw. Kapitalgewinne gibt. Die Akteure, die in der Periode des Budgetdefizits jung sind, profitieren in beiden Ländern vom Budgetdefizit. Der Grund ist der, daß der Zinssatz auf dem Pfad hin zum neuen langfristigen Gleichgewicht monoton steigt, was einen positiven intertemporalen Terms of Trade Effekt bewirkt, und der Lohn vom Budgetdefizit unberührt bleibt, da er vom existierenden Kapitalstock determiniert wird. Zusätzlich profitieren die Akteure des Landes, in dem das staatliche Budgetdefizit auftritt, durch den ihnen zukommenden Transfer.

Im hier entwickelten Modell erhöht sich oder reduziert sich die Wohlfahrt des ausländischen Akteurs 1, je nachdem, ob der kurzfristige Zinssatz im Übergang hin zum neuen langfristigen Gleichgewicht im Verhältnis zum Zinssatz im alten langfristigen Gleichgewicht steigt oder sinkt. Die Wohlfahrt der Akteure, die in der Periode des Budgetdefizits jung sind, wird wie im Diamond-Modell in beiden Ländern genau dann erhöht, wenn der Zinssatz steigt. Daß der Zinssatz fällt

' Siehe dazu Persson (1985), S. 80ff. 
und somit die Akteure in beiden Ländern gegenläufig betroffen sind, ist im Diamond-Modell nicht möglich.

\subsection{Die Entwicklung der Zahlungsbilanz}

Zwar befindet sich die integrierte Weltwirtschaft ab Periode 2 in ihrem neuen langfristigen Gleichgewicht, der Allokationsvektor und der Preis der Vermögenstitel haben ihren neuen stationären Wert angenommen, die Zahlungsbilanz dieser Periode wird allerdings noch von der Übergangsdynamik beeinflußt. In der Zahlungsbilanz werden alle innerhalb einer Periode stattfindenden ökonomischen Transaktionen zwischen inländischen und ausländischen Wirtschaftseinheiten statistisch erfaßt. ${ }^{1}$ Im folgenden interessiert die Entwicklung der Leistungsbilanz und die der Handelsbilanz.

Die Leistungsbilanz eines Landes gibt Auskunft über die zwischen zwei Perioden stattfindende Veränderung seines Nettoauslandsvermögens. Die Leistungsbilanz der Periode $t+1$ ist hierbei als Differenz des Nettoauslandsvermögens der Periode $t+1$ und dem der Periode $t$ definiert. $^{2}$ Die Leistungsbilanz weist einen Überschuß auf, wenn das Land ein Nettokreditgeber ist. Sie weist ein Defizit auf, wenn das Land ein Nettokreditnehmer ist. Anders ausgedrückt, ist die Leistungsbilanz die Differenz zwischen dem Volkseinkommen und der inländischen Absorption oder einfach die Nettoauslandsinvestition. Das Volkseinkommen ist hierbei die Summe aus dem inländischen Produktionsergebnis (die Inlandsproduktion) und dem aus dem bestehenden Nettoauslandsvermögen erzielten Faktoreinkommen.

${ }^{1}$ Vgl. z.B. Siebert (1991), S.191ff.

${ }^{2}$ Akteur $t$ erwirbt in Periode $t$ einen Anteil an der ausländischen Produktionstechnik. In Periode $t+1$ überträgt er diesen durch Einlösung des Schuldscheins wieder zurück. In derselben Periode erwirbt Akteur $t+1$ gegebenenfalls einen Anteil an der ausländischen Produktionstechnik. Je nachdem, ob der von Akteur $t+1$ erworbene Anteil größer oder kleiner als der von Akteur $t$ zurückgegebene ist, erhöht sich oder senkt sich das Nettoauslandsvermögen in Periode $t+1$. 
In der Handelsbilanz werden die innerhalb einer Periode stattfindenden Güterexporte und Güterimporte gegenübergestellt. Die Handelsbilanz eines Landes weist einen Überschuß auf, wenn es mehr Güter exportiert als importiert (man spricht auch von einer aktiven Handelsbilanz). Die Handelsbilanz weist ein Defizit auf, wenn das Land mehr Güter importiert als exportiert (man spricht auch von einer passiven Handelsbilanz). Anders ausgedrückt ist die Handelsbilanz die Differenz zwischen Inlandsproduktion und inländischer Absorption. ${ }^{1}$

Da ein Zwei-Länder-Modell betrachtet wird, ist der Leistungsbilanzüberschuß eines Landes gleichzeitig das Leistungsbilanzdefizit des anderen Landes, und der Handelsbilanzüberschuß des einen Landes ist gleichzeitig das Handelsbilanzdefizit des anderen Landes.

\subsubsection{Die Entwicklung der Zahlungsbilanz bei}

\section{inländischer Staatsverschuldung}

In diesem Abschnitt soll die aus einem inländischen Budgetdefizit folgende Dynamik der Zahlungsbilanz untersucht werden. Es wird also die Entwicklung des Abschnitts 6.2 betrachtet, also der Fall, in dem sich der Staat des Landes verschuldet, welches von den Akteuren mit der niedrigeren Zeitpräferenz bevölkert wird.

Die Leistungsbilanz der Periode 1 hängt davon ab, ob der kurzfristige Zinssatz in der folgenden Periode über sein altes langfristiges Niveau steigt oder unter diesem verbleibt. Im Fall, da $\beta \bar{r}_{0} \leq \bar{r}_{2} \Leftrightarrow \bar{\delta}_{0} \geq \bar{\delta}_{2}$ gilt, weist die Zahlungsbilanz des Auslands in der Periode 1 einen Leistungsbilanzüberschuß auf bzw. die des Inlands ein Leistungsbilanzdefizit. Der Grund ist folgender: Da $\bar{\delta}_{0} \geq \bar{\delta}_{2}$ gilt, und die Güter Bruttosubstitute sind, ist die gewünschte Ersparnis des ausländischen

${ }^{1}$ Die Handelsbilanz stellt somit auf den Handel innerhalb einer Periode ab. Dies ist eine zeitpunktbezogene Betrachtungsweise. Die Leistungsbilanz dagegen resultiert aus einer zeitraumbezogenen Betrachtungsweise. Sie stellt auf die zwischen zwei Perioden stattfindende Veränderung der Auslandsposition ab. Siehe auch Obstfeld und Rogoff (1996), S. 5f, zu diesem Themenkomplex. 
Akteurs 1 mindestens so hoch wie die Ersparnis des ausländischen Akteurs 0. ${ }^{1}$ In Verbindung mit $\bar{p}_{0}>\bar{p}_{1}$ folgt, daß der Anteil an der ausländischen Produktionstechnik, den der ausländische Akteur 1 erwerben möchte, größer ist als der vom ausländischen Akteur 0 gehaltene. Somit reduziert sich der von den Inländern gehaltene Anteil an der ausländischen Produktionstechnik in Periode 1 gegenüber dem Anteil der Periode 0. Die Zahlungsbilanz des Auslands weist in Periode 1 somit einen Leistungsbilanzüberschuß auf bzw. die des Inlandes ein Leistungsbilanzdefizit. ${ }^{2}$ Im Fall, daß $\bar{r}_{0}>\bar{r}_{2} \Leftrightarrow \bar{\delta}_{0}<\bar{\delta}_{2}$ gilt, läßt sich keine eindeutige Aussage über die Struktur der Leistungsbilanz in Periode 1 machen: Zwar ist nun die gewünschte Ersparnis des ausländischen Akteurs 1 wegen $\bar{\delta}_{0}<\bar{\delta}_{2}$ und der Bruttosubstitut-Eigenschaft der Güter geringer als die Ersparnis des ausländischen Akteurs 0 , wegen $\bar{p}_{0}>\bar{p}_{1}$ ist es dennoch möglich, daß der Anteil an der ausländischen Produktionstechnik, den der ausländische Akteur 1 erwerben möchte, größer als der vom ausländischen Akteur 0 gehaltene ist.

Da $\bar{\delta}_{2}>\bar{\delta}_{3}$ gilt, und die Güter Bruttosubstitute sind, muß die gewünschte Ersparnis des ausländischen Akteurs 2 höher sein als die Ersparnis des ausländischen Akteurs 1. In Verbindung mit $\bar{p}_{1}>\bar{p}_{2}$ folgt, daß der Anteil an der ausländischen Produktionstechnik, den der ausländische Akteur 2 erwerben möchte, größer ist, als der vom ausländischen Akteur 1 gehaltene. Somit reduziert sich der von den Inländern gehaltene Anteil an der ausländischen Produktionstechnik in Periode 2 gegenüber dem Anteil der Periode 1. Die Zahlungsbilanz des Auslands weist in Periode 2 somit einen Leistungsbilanzüberschuß auf bzw. die des Inlands ein Leistungsbilanzdefizit.

Ab Periode 3 ist die Leistungsbilanz wieder ausgeglichen. Die Auslandsinvestition des Inlands hat sich gegenüber der Situation ohne inländische Staatsverschuldung reduziert, ist aber ab Periode 2 konstant. Entsprechend hat sich der

\footnotetext{
${ }^{1}$ Generation 1 erwartet für Periode 1 den Diskontfaktor $\bar{\delta}_{0}$, da sie das staatliche Budgetdefizit der Periode 1 nicht antizipiert.

${ }^{2}$ Die Nettoauslandsinvestition des Auslands in Periode 1 ist positiv und die des Inlands ist negativ (die Auslandsinvestition des Inländers 1 ist niedriger als die des Inländers 0 ). $\mathrm{Be}-$ merkbar macht sich das Leistungsbilanzungleichgewicht durch eine Veränderung des von den Inländern gehaltenen Anteils an der ausländischen Produktionstechnik. Das $\bar{x}$ der Periode 1 ist kleiner als das der Periode 0.
} 
ausländische Handelsbilanzüberschuß gegenüber der Situation ohne inländische Staatsverschuldung reduziert. Auch ist der ausländische Handelsbilanzüberschuß der Periode 3 geringer als der in Periode 2. Der Grund liegt in der gegenüber Periode 1 verringerten Auslandsinvestition des Inlands in Periode 2. Es gilt:

$$
\begin{aligned}
& \underbrace{\bar{x}_{1} \cdot\left(\bar{p}_{2}+\varepsilon\right)-\bar{x}_{2} \cdot \bar{p}_{2}}_{\text {ausl. Handelsbilanz Periode } 2}>\underbrace{\bar{x}_{2} \cdot\left(\bar{p}_{3}+\varepsilon\right)-\bar{x}_{3} \cdot \bar{p}_{3}}_{\text {ausl. Handelsbilanz Periode } 3}, \\
& \text { da } \bar{x}_{1}>\bar{x}_{2}=\bar{x}_{3} \text { und } \bar{p}_{2}=\bar{p}_{3} .
\end{aligned}
$$

$\bar{x}_{t}$ ist hierbei der Anteil an der ausländischen Produktionstechnik, den der inländische Akteur $t$ durch den Kauf des durch den ausländischen Akteur $t$ emittierten Schuldscheins erwirbt.

Im Fall, daß die Zahlungsbilanz des Auslands in Periode 1 einen Leistungsbilanzüberschuß aufweist bzw. die des Inlands ein Leistungsbilanzdefizit, besitzt das Ausland in dieser Periode einen Handelsbilanzüberschuß, der höher ist als der im neuen langfristigen Gleichgewicht. Es gilt:

$$
\begin{aligned}
& \underbrace{\bar{x}_{0} \cdot\left(\bar{p}_{1}+\varepsilon\right)-\bar{x}_{1} \cdot \bar{p}_{1}}_{\text {ausl. Handelsbilanz Periode } 1}>\underbrace{\bar{x}_{2} \cdot\left(\bar{p}_{3}+\varepsilon\right)-\bar{x}_{2} \cdot \bar{p}_{3}}_{\text {ausl. Handelsbilanz Periode } 3}, \\
& \text { da } \bar{x}_{0}>\bar{x}_{1}>\bar{x}_{2}=\bar{x}_{3} \text { und } \bar{p}_{1}>\bar{p}_{3} .
\end{aligned}
$$

Das Modell macht aber keine Aussagen darüber, ob der Handelsbilanzüberschuß in Periode 1 höher oder niedriger als der in Periode 2 ist. Im Fall, daß die Zahlungsbilanz des Auslands in Periode 1 ein Leistungsbilanzdefizit aufweist bzw. die des Inlands einen Leistungsbilanzüberschuß, besitzt das Ausland in dieser Periode einen Handelsbilanzsaldo, der geringer ist als der in Periode 2. Hier gilt:

$$
\begin{aligned}
& \underbrace{\bar{x}_{0} \cdot\left(\bar{p}_{1}+\varepsilon\right)-\bar{x}_{1} \cdot \bar{p}_{1}}_{\text {ausl. Handelsbilanz Periode } 1}<\underbrace{\bar{x}_{1} \cdot\left(\bar{p}_{2}+\varepsilon\right)-\bar{x}_{2} \cdot \bar{p}_{2}}_{\text {ausl. Handelsbilanz Periode } 2}, \\
& \text { da } \bar{x}_{1}>\bar{x}_{0}>\bar{x}_{2} \text { und } \bar{p}_{1}>\bar{p}_{2} .
\end{aligned}
$$


Das Modell macht aber keine Aussagen darüber, ob der Saldo der Handelsbilanz in Periode 1 größer oder kleiner ist als der im neuen langfristigen Gleichgewicht. Es ist sogar möglich, daß die positive Nettoauslandsinvestition des Inlands in Periode 1 ein Handelsbilanzdefizit des Auslands bewirkt. Die Bedingung hierfür ist $\bar{x}_{0} \cdot\left(\bar{p}_{1}+\varepsilon\right)<\bar{x}_{1} \cdot \bar{p}_{1} \Leftrightarrow \bar{x}_{0} / \bar{x}_{1}<\bar{p}_{1} /\left(\bar{p}_{1}+\varepsilon\right)$.

\subsubsection{Die Entwicklung der Zahlungsbilanz bei}

\section{ausländischer Staatsverschuldung}

Die Entwicklung der Zahlungsbilanz, die resultiert, wenn sich der Staat des Landes verschuldet, dessen Akteure im Verhältnis zu den Akteuren des anderen Landes relativ ungeduldig sind, sieht wie folgt aus: Im Fall, daß $\bar{r}_{0} \leq \bar{r}_{2} \Leftrightarrow \bar{\delta}_{0}$ $\geq \bar{\delta}_{2}$ gilt, weist nun die Zahlungsbilanz des Inlands in Periode 1 einen Leistungsbilanzüberschuß auf bzw. die des Auslands ein Leistungsbilanzdefizit. Der Grund ist folgender: In Verbindung mit der Bruttosubstitut-Eigenschaft der Güter folgt aus $\bar{\delta}_{0} \geq \bar{\delta}_{2}$, daß die gewünschte Ersparnis des inländischen Akteurs 1 mindestens so hoch ist wie die Ersparnis des inländischen Akteurs 0. In Verbindung mit $\bar{p}_{0}>\bar{p}_{1}$ folgt dann, daß der Anteil an der ausländischen Produktionstechnik, den der inländische Akteur 1 erwerben möchte, größer ist als der vom inländischen Akteur 0 gehaltene. Somit erhöht sich der von den Inländern gehaltene Anteil an der ausländischen Produktionstechnik in Periode 1 gegenüber dem Anteil der Periode 0. Die Zahlungsbilanz des Inlands weist in Periode 1 somit einen Leistungsbilanzüberschuß auf bzw. die des Auslands ein Leistungsbilanzdefizit. Im Fall, da $\bar{r}_{0}>\bar{r}_{2} \Leftrightarrow \bar{\delta}_{0}<\bar{\delta}_{2}$ gilt, läßt sich wieder keine eindeutige Aussage über die Struktur der Leistungsbilanz in Periode 1 machen: Die gewünschte Ersparnis des inländischen Akteurs 1 ist wegen $\bar{\delta}_{0}<\bar{\delta}_{2}$ und der Bruttosubstitut-Eigenschaft der Güter geringer als die Ersparnis des inländischen Akteurs 0 . Wegen $\bar{p}_{0}>\bar{p}_{1}$ ist es dennoch möglich, daß der Anteil an der ausländischen Produktionstechnik, den der inländische Akteur 1 erwerben möchte, größer als der vom inländischen Akteur 0 gehaltene ist.

In Periode 2 weist die Zahlungsbilanz des Inlands einen Leistungsbilanzüberschuß auf bzw. die des Auslands ein Leistungsbilanzdefizit. Der Grund liegt in 
$\bar{\delta}_{2}>\bar{\delta}_{3}$ und $\bar{p}_{1}>\bar{p}_{2}$ in Verbindung mit der Bruttosubstitut-Eigenschaft der Güter: Wegen $\bar{\delta}_{2}>\bar{\delta}_{3}$ und der Bruttosubstitut-Eigenschaft ist die gewünschte Ersparnis des inländischen Akteurs 2 höher als die Ersparnis des inländischen Akteurs 1. In Verbindung mit $\bar{p}_{1}>\bar{p}_{2}$ folgt, daß der Anteil an der ausländischen Produktionstechnik, den der inländische Akteur 2 erwerben möchte, größer als der vom inländischen Akteur 1 gehaltene ist. Der von den Inländern gehaltene Anteil an der ausländischen Produktionstechnik erhöht sich somit in Periode 2 gegenüber dem Anteil in Periodel.

Ab Periode 3 ist die Leistungsbilanz wieder ausgeglichen. Die Auslandsinvestition des Inlands hat sich gegenüber der Situation ohne ausländische Staatsverschuldung erhöht, ist aber ab Periode 2 konstant. Der ausländische Handelsbilanzüberschuß hat sich gegenüber der Situation ohne ausländische Staatsverschuldung entsprechend erhöht. Er ist ab Periode 3 konstant und höher als der Handelsbilanzsaldo in Periode 2. Der Grund liegt in der gegenüber der Periode 1 erhöhten Auslandsinvestition des Inlands. Es gilt also:

$$
\begin{aligned}
\underbrace{\bar{x}_{1} \cdot\left(\bar{p}_{2}+\varepsilon\right)-\bar{x}_{2} \cdot \bar{p}_{2}}_{\text {ausl. Handelsbilanz Periode } 2}<\underbrace{\bar{x}_{2} \cdot\left(\bar{p}_{3}+\varepsilon\right)-\bar{x}_{3} \cdot \bar{p}_{3}}_{\text {ausl. Handelsbilanz Periode } 3}, \\
\text { da } \bar{x}_{1}<\bar{x}_{2}=\bar{x}_{3} \text { und } \bar{p}_{2}=\bar{p}_{3} .
\end{aligned}
$$

Im Fall, daß die Zahlungsbilanz des Inlands in Periode 1 einen Leistungsbilanzüberschuß bzw. die des Auslands ein Leistungsbilanzdefizit aufweist, besitzt das Ausland in Periode 1 einen Saldo der Handelsbilanz, der geringer ist als der im neuen langfristigen Gleichgewicht. Es gilt:

$$
\begin{aligned}
& \underbrace{\bar{x}_{0} \cdot\left(\bar{p}_{1}+\varepsilon\right)-\bar{x}_{1} \cdot \bar{p}_{1}}_{\text {ausl. Handelsbilanz Periode } 1}<\underbrace{\bar{x}_{2} \cdot\left(\bar{p}_{3}+\varepsilon\right)-\bar{x}_{3} \cdot \bar{p}_{3}}_{\text {ausl. Handelsbilanz Periode } 3}, \\
& \text { da } \bar{x}_{0}<\bar{x}_{1}<\bar{x}_{2}=\bar{x}_{3} \text { und } \bar{p}_{1}>\bar{p}_{3} .
\end{aligned}
$$

Das Modell macht aber keine Aussagen darüber, ob der Saldo der Handelsbilanz in Periode 1 größer oder kleiner ist als der in Periode 2: Durch die positiven Nettoauslandsinvestitionen des Inlands in den Perioden 1 und 2 ist es möglich, daß die Handelsbilanz des Auslands in diesen Perioden ein Defizit auf- 
weist. In Periode 1 ist die Bedingung hierfür $\bar{x}_{0} \cdot\left(\bar{p}_{1}+\varepsilon\right)<\bar{x}_{1} \cdot \bar{p}_{1} \Leftrightarrow \bar{x}_{0} / \bar{x}_{1}$ $<\bar{p}_{1} /\left(\bar{p}_{1}+\varepsilon\right)$ und in Periode $2 \bar{x}_{1} \cdot\left(\bar{p}_{2}+\varepsilon\right)<\bar{x}_{2} \cdot \bar{p}_{2} \Leftrightarrow \bar{x}_{1} / \bar{x}_{2}<\bar{p}_{2} /\left(\bar{p}_{2}+\varepsilon\right)$.

Im Fall, daß die Zahlungsbilanz des Inlands in Periode 1 ein Leistungsbilanzdefizit aufweist bzw. die des Auslands einen Leistungsbilanzüberschuß, besitzt das Ausland in Periode 1 einen Handelsbilanzüberschuß der höher ist als der Handelsbilanzsaldo in Periode 2. Es gilt:

$$
\begin{aligned}
\underbrace{\bar{x}_{0} \cdot\left(\bar{p}_{1}+\varepsilon\right)-\bar{x}_{1} \cdot \bar{p}_{1}}_{\text {ausl. Handelsbilanz Periode } 1}>\underbrace{\bar{x}_{1} \cdot\left(\bar{p}_{2}+\varepsilon\right)-\bar{x}_{2} \cdot \bar{p}_{2}}_{\text {ausl. Handelsbilanz Periode } 2}, \\
\text { da } \bar{x}_{1}<\bar{x}_{0}<\bar{x}_{2} \text { und } \bar{p}_{1}>\bar{p}_{2} .
\end{aligned}
$$

Das Modell macht aber keine Aussage darüber, ob der ausländische Handelsbilanzüberschuß in Periode 1 höher oder niedriger ist als der im neuen langfristigen Gleichgewicht.

Vergleicht man die Ergebnisse aus den Unterabschnitten 6.3.1 und 6.3.2 miteinander so erkennt man, daß es für die Struktur der durch ein staatliches Budgetdefizit induzierten Leistungsbilanzdynamik unerheblich ist, ob dieses in einem Schuldnerland oder in einem Gläubigerland auftritt. Für die Struktur, der durch ein staatliches Budgetdefizit induzierten Handelsbilanzdynamik, ist die Auslandsposition des betreffenden Landes allerdings von entscheidender Bedeutung. 
Michael Ganske - 978-3-631-75472-6

Downloaded from PubFactory at 01/11/2019 04:35:58AM

via free access 


\section{Zusammenfassung und Fazit}

Im Verlauf der Abhandlung wurden OLG-Modelle der Weltwirtschaft diskutiert, anhand derer intertemporale Aspekte der Finanzierungsentscheidung des Staates und des Außenhandels aufgezeigt wurden. Den in den Kapiteln 3 bis 6 entwickelten Modellen wurde bewußt nur die Struktur gegeben, die für das Vorhaben notwendig war. Es gibt zum Beispiel keine intratemporalen Relativpreise wie den Wechselkurs (also das Verhältnis der nationalen Preisniveaus) oder die intratemporalen Terms of Trade (also der Relativpreis von Importen ausgedrückt in Exporten), sondern nur die intertemporalen Terms of Trade (also die Zinssätze). Die Produktionsseite wurde auf eine Art und Weise modelliert, die eine Diskussion der Dynamik der Zinsstruktur ermöglicht (es gibt in jeder Periode einen kurzfristigen und einen langfristigen Zinssatz). Auch wurden die Länderunterschiede in einer intertemporalen Dimension formuliert. So wurde angenommen, daß sich die beiden die Weltwirtschaft konstituierenden Länder nur bezüglich der Zeitpräferenz der sie bevölkernden Akteure unterscheiden. Diese Annahme führte, wie schon in Kapitel 1 darauf hingewiesen wurde, zur Verwendung des OLG-Modells und machte die Verwendung des RA-Modells wenig sinnvoll. ${ }^{1}$

Abschnitt 7.1 beinhaltet eine komprimierte Darstellung der in den Kapiteln 3 bis 6 hergeleiteten Resultate. In Abschnitt 7.2 wird dann ein Fazit aus den in der Abhandlung durchgeführten Analysen gezogen.

\footnotetext{
' Ganz abgesehen davon, daß im RA-Modell das Ricardo-Äquivalenztheorem Gültigkeit besitzt und damit keine sinnvolle Diskussion der Auswirkungen staatlicher Budgetdefizite möglich ist. Die Notwendigkeit der Verwendung des OLG-Modells ist allerdings nur dann gegeben, wenn man sich auf mikroökonomisch fundierte makroökonomische Modelle beschränkt. Dies sollte man aber tun, nimmt man die Lucas-Kritik ernst. Lucas (1976) zeigt, daß wenn die Erwartungen rational gebildet werden, die Bewertung von politischen Maßnahmen nur dann korrekt ist, wenn das verwendete Modell nur aus wirklichen Struktur- und Verhaltensgleichungen besteht; vgl. Lucas (1976), S. 24ff. Eine mögliche Konsequenz aus der Lucas-Kritik besteht darin, Modelle zu verwenden, deren einzige als invariant gegenüber alternativen Politikregimen angenommenen Parameter jene sind, welche die Tiefenstruktur (also Präferenzen und Technologie) determinieren. Diese führt zur Verwendung von Optimierungsmodellen. Siehe dazu auch Minford (1992), S. 87ff.
} 


\subsection{Zusammenfassung}

In Kapitel 3 wurde der Modellrahmen entwickelt, innerhalb dessen die in den folgenden Kapiteln durchgeführte Analyse stattfand. Es zeigte sich, daß zwei Fälle zu unterscheiden sind: Entweder das Modell ist regulär, dann besitzt die Ökonomie nur einen Gleichgewichtspfad (sie muß sich von Anfang an im langfristigen Gleichgewicht befinden und dieses ist instabil), oder es ist irregulär, dann besitzt die Ökonomie unendlich viele Gleichgewichtspfade, die in der Nähe des langfristigen Gleichgewichts beginnen und zu diesem hin konvergieren (das langfristige Gleichgewicht ist in diesem Fall lokal asymptotisch stabil und indeterminiert). Ursache für die Irregularität des Modells ist der Einkommenseffekt, der den intertemporalen Substitutionseffekt in der Jugend so stark überkompensiert, daß die Steigung der Tauschkurve im langfristigen Gleichgewicht größer als eins ist. Es ist sinnvoll bei der Diskussion der Auswirkungen der Staatsverschuldung und des Außenhandels den regulären Fall zu betrachten. Im irregulären Fall ist keine Aussage darüber zu treffen, in welchem Zustand sich die Ökonomie unmittelbar nach einem Strukturbruch (nicht antizipiertes Budgetdefizit oder nicht antizipierte Öffnung zum Weltmarkt) befindet, da in diesem Fall unendlich viele Folgen nicht stationärer Gleichgewichte, die hin zum neuen langfristigen Gleichgewicht konvergieren, existieren. Unabhängig von der Regularität des Modells ist das langfristige Gleichgewicht intertemporal optimal. Die Zinsdynamik, die im irregulären Fall auf dem Weg hin zum langfristigen Gleichgewicht resultiert, weist abwechselnd Perioden inverser und normaler Zinsstruktur auf, wobei die Zinsspreizung immer geringer wird je näher die Ökonomie an ihrem langfristigen Gleichgewicht ist. Im irregulären Fall ist die Existenz zyklischer Gleichgewichte möglich, was am Beispiel eines zweiperiodigen Zyklus exemplifiziert wurde. Auch der zweiperiodige Zyklus ist intertemporal optimal, und in ihm wechseln sich Perioden inverser und normaler Zinsstruktur ab. 


\subsubsection{Staatsverschuldung in der geschlossenen Ökonomie}

In Kapitel 4 wurde ein Staat in das Modell eingeführt, der Umverteilungspolitik betreibt. Der staatliche Transfer wird hierbei durch den Verkauf von ewigen Staatsanleihen finanziert, welche im Portfolio der Anleger vollkommene Substitute zum Anteilschein an der Produktionstechnik darstellen. Der mit den emittierten Staatsanleihen verbundene Zinsendienst wird durch die Besteuerung des Arbeitseinkommens der zukünftigen Generationen bestritten, deren verfügbares Einkommen sich dadurch reduziert.

\section{Permanente Wirkungen}

Die langfristigen Auswirkungen der Staatsverschuldung sind die Erhöhung des Zinssatzes, eine Reduktion des Jugendkonsums und eine Erhöhung des Alterskonsums, sowie die Reduktion der Wohlfahrt der Akteure. Zwar bewirkt die Staatsverschuldung durch die Erhöhung des Zinssatzes einen positiven intertemporalen Terms of Trade Effekt (die Verzinsung der Ersparnis erhöht sich), die aus der Besteuerung resultierende Reduktion des in der Jugend verfügbaren Einkommens überkompensiert allerdings den Terms of Trade Effekt in seiner Wohlfahrtswirkung.

\section{Transitorische Wirkungen}

Die in Folge eines aus einem nicht antizipierten staatlichen Budgetdefizit resultierende Anpassungsdynamik der Ökonomie sieht wie folgt aus: Die Emission der Staatsanleihen erzeugt einen Kapitalverlust, den der in der Periode des Budgetdefizits alte Akteur hinnehmen muß. Der kurzfristige Zinssatz sinkt in dieser Periode unter den Zinssatz im alten langfristigen Gleichgewicht, und der von ihm gehaltene Anteilschein verliert an Wert. Ob der kurzfristige Zinssatz in der folgenden Periode unter den Zinssatz im alten langfristigen Gleichgewicht fällt oder über diesen steigt, hängt vom Verlauf des Einkommensexpansionspfades der Akteure ab. Beide Fälle sind möglich. Im ersten Fall kann es sogar sein, daß der kurzfristige Zinssatz unter das Niveau absinkt, das er in der Periode des 
Budgetdefizits hat. Erst zwei Perioden nach Auftreten des Budgetdefizits nimmt der Zinssatz seinen neuen durch die Staatsverschuldung erhöhten stationären Wert ein.

Der langfristige Zinssatz (der Kapitalmarktzins) steigt auf dem Pfad hin zum neuen langfristigen Gleichgewicht monoton an, und da sich der langfristige Zinssatz und der Preis der Vermögenstitel invers zueinander verhalten, sinkt letzterer monoton. Der Preis der Vermögenstitel nimmt schon eine Periode nach Auftreten des Budgetdefizits seinen neuen stationären Wert an. Die Ökonomie befindet sich ab dieser Periode im neuen langfristigen Gleichgewicht und der langfristige Zinssatz zwei Perioden danach. Für die Zinsdynamik bedeutet dies, daß die Zinsstruktur in der Periode des Budgetdefizits und in der ihr folgenden normal ist, zwei Perioden nach Auftreten des Budgetdefizits ist sie wieder flach.

Die Wohlfahrt des Akteurs, der in der Periode des Budgetdefizits alt ist, wird durch den Kapitalverlust, den er hinnehmen muß, reduziert. Sein Alterskonsum nimmt ab. Die Wohlfahrt des Akteurs, dem der Transfer zugute kommt, erhöht sich. Sein Konsum ist in beiden Lebensabschnitten höher als der Konsum der Akteure im alten langfristigen Gleichgewicht. Wie die Analyse der langfristigen Auswirkungen der Staatsverschuldung gezeigt hat, reduziert sich die Wohlfahrt der folgenden Generationen. Dabei hat sich ihr Jugendkonsum gegenüber dem Jugendkonsum der Akteure im Gleichgewicht ohne Staatsverschuldung reduziert, und der Alterskonsum hat sich erhöht.

\subsubsection{Außenhandel}

In Kapitel 5 wurde das Modell auf zwei Länder erweitert. Dabei wurde angenommen, daß sich das Inland und das Ausland nur bezüglich der Zeitpräferenz der sie bevölkernden Akteure unterscheiden. Die Ausländer sind per Annahme ungeduldiger als die Inländer, sie besitzen eine höhere Zeitpräferenz. Aus diesem Grund ist der ausländische Autarkiezinssatz höher als der inländische. 


\section{Permanente Wirkungen}

Öffnen sich beide Länder dem Weltmarkt, so liegt der Zinssatz im langfristigen Gleichgewicht der integrierten Weltwirtschaft zwischen den nationalen Autarkiezinssätzen. Aus Sicht der Inländer erhöht sich der Zinssatz langfristig durch die Integration, aus Sicht der Ausländer sinkt er. Verglichen mit den Autarkiegleichgewichten erhöht sich der Alterskonsum im Inland und reduziert sich im Ausland. Sind der Konsum in der Jugend und im Alter im Inland Bruttosubstitute, dann sinkt hier der Jugendkonsum im Vergleich zum Autarkiegleichgewicht. Sind sie Bruttokomplemente, dann steigt er. Im Ausland gilt das genau umgekehrte: Hier erhöht sich der Jugendkonsum bei Bruttosubstituten und senkt sich bei Bruttokomplementen. Im Sonderfall, daß der Preis der Vermögenstitel in der integrierten Weltwirtschaft gleich dem arithmetischen Mittel der Autarkiepreise ist, unterscheiden sich der Weltjugendkonsum und der Weltalterskonsum bei Autarkie nicht von denen bei Freihandel.

Damit die gerade beschriebene Gleichgewichtsallokation erreicht werden kann ist es notwendig, daß die Inländer Auslandsinvestitionen tätigen. Sie erwerben ein Teileigentum an der ausländischen Produktionstechnik. Hierfür emittieren die jungen Ausländer in jeder Periode Schuldscheine, die nach einer Periode eingelöst werden. Zwar entsteht somit jede Periode eine neue Kreditbeziehung zwischen den Akteuren einer Generation, makroökonomisch betrachtet erzeugt dies aber eine konstante Auslandsinvestition des Inlands. Das Inland ist somit ein Gläubigerland, das Ausland ein Schuldnerland. Diese Tatsache bedingt, daß die Handelsbilanz des Auslands in jeder Periode einen Überschuß aufweist bzw. die des Inlands ein Defizit. Das Handelsbilanzungleichgewicht entspricht hierbei der Gewinnbeteiligung der Inländer an der ausländischen Produktionstechnik.

Die Wohlfahrt der Inländer erhöht sich durch die weltwirtschaftliche Integration, die der Ausländer reduziert sich. Die intertemporalen Terms of Trade der Inländer verbessern sich (aus ihrer Sicht steigt die Verzinsung der Ersparnis) und die der Ausländer verschlechtern sich (aus ihrer Sicht reduziert sich die Verzinsung der Ersparnis). Abgesehen davon, reduziert sich die im Ausland in 
jeder Periode verfügbare Gütermenge durch den Handelsbilanzüberschuß bzw. erhöht sich im Inland.

\section{Transitorische Wirkungen}

Die Auswirkungen der Öffnung der Ökonomien hin zum Weltmarkt sehen wie folgt aus: Schon in der Periode der von den Akteuren nicht antizipierten Öffnung investiert der junge Inländer im Ausland, in dem er den vom jungen Ausländer emittierten Schuldschein erwirbt. Ab dieser Periode werden die Vermögenstitel zu einem auf dem Weltkapitalmarkt determinierten Preis gehandelt. Der Preis ist dabei schon in der Öffnungsperiode auf dem Niveau, das er im langfristigen Gleichgewicht der integrierten Weltwirtschaft annimmt. Aus Sicht der Inländer hat sich der Preis durch die Öffnung reduziert, aus Sicht der Ausländer hat er sich erhöht. Eine Periode nach Öffnung zum Weltmarkt befindet sich die integrierte Weltwirtschaft in ihrem langfristigen Gleichgewicht.

Aus inländischer Sicht sinkt der kurzfristige Zinssatz in der Periode der Öffnung unter den Zinssatz im Autarkiegleichgewicht. Schon eine Periode danach nimmt er seinen neuen langfristigen erhöhten Wert an. Der langfristige Zinssatz ist bis einschließlich der Periode der Öffnung auf seinem Autarkieniveau, und eine Periode nach der Öffnung nimmt er seinen neuen erhöhten langfristigen Wert an. Die Zinsstruktur ist somit in der Periode der Öffnung normal, danach ist sie wieder flach.

Aus ausländischer Sicht steigt der kurzfristige Zinssatz in der Periode der Öffnung über sein Autarkieniveau. In der folgenden Periode sinkt er auf seinen neuen langfristigen reduzierten Wert. Der langfristige Zinssatz ist bis einschließlich der Öffnungsperiode auf seinem Autarkieniveau und sinkt in der der Öffnung folgenden Periode auf seinen neuen langfristigen Wert. Die Zinsstruktur ist in der Öffnungsperiode somit invers und danach wieder flach. Folglich stimmen die Zinssätze erst eine Periode nach der Integration weltweit überein.

Über die Wohlfahrt der von der Integration betroffenen Generationen läßt sich folgendes sagen: Der in der Periode der Öffnung alte Inländer muß einen Kapi- 
talverlust hinnehmen, da der von ihm gehaltene Anteilschein an Kurswert verliert. Der alte Ausländer profitiert von einem Kapitalgewinn, da sich der Kurswert des von ihm gehaltenen Anteilscheins aus seiner Sicht erhöht. Der Alterskonsum des Inländers nimmt gegenüber der Autarkiesituation ab, seine Wohlfahrt reduziert sich also, der des Ausländers nimmt zu, seine Wohlfahrt erhöht sich damit. Die Wohlfahrt der in der Öffnungsperiode jungen Akteure entspricht derjenigen der Akteure der folgenden Generationen. Die Wohlfahrt der Inländer erhöht sich und die der Ausländer reduziert sich bedingt durch die weltwirtschaftliche Integration. Als Folge ist das Verhältnis von Gewinnern und Verlierern in der Öffnungsperiode in den beiden Ländern ausgeglichen.

\subsubsection{Staatsverschuldung in der integrierten Weltwirtschaft}

In Kapitel 6 wurden die Auswirkungen der Staatsverschuldung in der integrierten Weltwirtschaft analysiert. Es wurde hierbei untersucht, wie sich die Verschuldung des inländischen Staates auf die Weltwirtschaft auswirkt. Hierfür wurde angenommen, daß sich der ausländische Staat völlig passiv verhält, sein Haushalt also in jeder Periode ein ausgeglichenes Budget aufweist. Wenn die Eigenschaft der relativen Zeitpräferenz der Akteure des Landes, dessen Staat sich verschuldet, für die Resultate von Bedeutung ist, wurde auch diskutiert, wie sich die ausländische Staatsverschuldung auswirken würde.

\section{Permanente Wirkungen}

Die langfristigen Auswirkungen der inländischen Staatsverschuldung in der integrierten Weltwirtschaft sehen wie folgt aus: Der Zinssatz erhöht sich wie im Fall der geschlossenen Ökonomie. Die Weltnachfrage der Jungen reduziert sich und die der Alten erhöht sich. Der inländische Jugendkonsum sinkt und der Alterskonsum sinkt oder steigt. Allerdings muß das Verhältnis von Jugendkonsum und Alterskonsum sinken, und die Summe aus beiden muß sich reduzieren. Der Alterskonsum der Ausländer erhöht sich, ihr Jugendkonsum reduziert sich bei Bruttosubstituten und erhöht sich bei Bruttokomplementen. Die Summe aus beiden muß sich aber erhöhen. Die Wohlfahrt der inländischen Akteure wird 
durch die inländische Staatsverschuldung reduziert, die der Ausländer wird erhöht.

Für die langfristige Handelsstruktur ist es von entscheidender Bedeutung, ob sich der Staat des Gläubigerlandes oder der des Schuldnerlandes verschuldet. Verschuldet sich der Staat des Gläubigerlandes, so reduzieren sich dessen Auslandsinvestitionen. Das Handelsbilanzungleichgewicht nimmt ab. Bei steigender Staatsverschuldung ist es möglich, daß das Gläubigerland zum Schuldnerland wird und vice versa. Das Handelsbilanzungleichgewicht nimmt dann wieder zu, allerdings mit umgekehrten Vorzeichen. Die öffentliche Verschuldung im Schuldnerland erhöht dessen Auslandsverschuldung und damit seinen Handelsbilanzüberschuß.

\section{Transitorische Wirkungen}

Im Folgenden sollen die Auswirkungen eines von den Akteuren nicht antizipierten inländischen staatlichen Budgetdefizits, welches als Folge eines dem inländischen Akteur zukommenden Transfers auftritt, zusammengefaßt werden. Um eine sinnvolle Diskussion der Dynamik zu ermöglichen wurde, wie schon im Fall der geschlossenen Ökonomie, die Annahme der Regularität des Modells verstärkt. Es wurde unterstellt, daß der Konsum in der Jugend und im Alter Bruttosubstitute sind. Die durch das Budgetdefizit induzierte Zinsdynamik stimmt in qualitativer Hinsicht mit der überein, die in der geschlossenen Ökonomie resultiert. Dieses Ergebnis ist ökonomisch plausibel, ist doch die integrierte Weltwirtschaft nichts anderes als eine einzelne geschlossene Ökonomie, mit zwei, sich durch die Zeitpräferenz unterscheidenden, Bevölkerungsgruppen.

\section{Die Zinssätze:}

Die Emission der inländischen Staatsanleihen erzeugt einen Kapitalverlust, den die in der Periode des Budgetdefizits alte Generation hinnehmen muß. Der kurzfristige Zinssatz dieser Periode sinkt unter sein altes langfristiges Niveau. Eine Aussage darüber, ob der kurzfristige Zinssatz der Folgeperiode höher oder niedriger als jener im alten langfristigen Gleichgewicht ist, ist nicht möglich. Im 
ersten Fall kann es sogar sein, daß er unter sein Niveau in der Periode des Budgetdefizits sinkt. Zwei Perioden nach dem Budgetdefizit nimmt der kurzfristige Zinssatz seinen neuen erhöhten stationären Wert an. Der langfristige Zinssatz steigt auf dem Pfad zu seinem neuen stationären Wert monoton an, bzw. der Preis der Vermögenstitel reduziert sich monoton. Die Zinsstruktur ist hierbei in der Periode des Budgetdefizits und in der ihr folgenden normal. In den nachfolgenden Perioden ist sie dann wieder flach. Der Preis der Vermögenstitel nimmt schon eine Periode nach Auftreten des Budgetdefizits seinen neuen reduzierten stationären Wert an.

\section{Die Wohlfahrt:}

Die Auswirkung auf die Wohlfahrt der vom inländischen Budgetdefizit betroffenen Generationen sieht folgendermaßen aus: Die Wohlfahrt der in der Periode des Budgetdefizits alten Akteure wird durch den Kapitalverlust reduziert. Die von ihnen gehaltenen Vermögenstitel verlieren an Kurswert, somit reduziert sich ihr Alterskonsum. Der inländische Akteur der Folgegeneration profitiert von dem ihm zukommenden Transfer. Sein Jugendkonsum und sein Alterskonsum erhöhen sich. Die Entwicklung der Wohlfahrt des ausländischen Akteurs derselben Generation hängt davon ab, ob der kurzfristige Zinssatz eine Periode nach auftreten des Budgetdefizits unter sein Niveau im alten langfristigen Gleichgewicht sinkt oder ob er über dieses steigt. Im ersten Fall verkleinert sich die Budgetmenge des ausländischen Akteurs gegenüber der Budgetmenge der ausländischen Akteure im langfristigen Gleichgewicht ohne Staatsverschuldung. Seine Wohlfahrt wird somit reduziert. Im zweiten Fall vergrößert sich seine Budgetmenge und seine Wohlfahrt wird erhöht. Die Wohlfahrt der Akteure der folgenden Generationen erhöht sich im Ausland und reduziert sich im Inland.

\section{Die Zahlungsbilanz:}

Die durch ein staatliches Budgetdefizit induzierte Zahlungsbilanzdynamik hängt davon $\mathrm{ab}$, ob dieses in einem Gläubigerland oder in einem Schuldnerland auftritt. Die Dynamik der Leistungsbilanz gibt hierbei die Entwicklung des 
Auslandsvermögens der Ökonomie wieder, die Dynamik der Handelsbilanz die Entwicklung des Außenhandels.

Verschuldet sich der Staat des Gläubigerlandes, sieht die Zahlungsbilanzdynamik wie folgt aus: Ist der kurzfristige Zinssatz eine Periode nach dem Budgetdefizit mindestens so hoch wie im alten langfristigen Gleichgewicht, so weist die Zahlungsbilanz des Gläubigerlandes in der Periode des Budgetdefizits ein Leistungsbilanzdefizit auf bzw. die des Schuldnerlandes einen Leistungsbilanzüberschuß. Im Fall, daß der Zinssatz unter seinem alten langfristigen Niveau verharrt, läßt sich keine Aussage über die Struktur der Leistungsbilanz treffen. Die Zahlungsbilanz der folgenden Periode weist im Gläubigerland ein Leistungsbilanzdefizit auf und im Schuldnerland einen Überschuß. Schon zwei Perioden nach Auftreten des Budgetdefizits ist die Leistungsbilanz wieder ausgeglichen.

Weist die Zahlungsbilanz des Gläubigerlandes in der Periode des Budgetdefizits ein Leistungsbilanzdefizit auf bzw. die des Schuldnerlandes einen Überschuß, besitzt letzteres in dieser Periode einen Handelsbilanzüberschuß, der höher ist als der im neuen langfristigen Gleichgewicht. Weist die Zahlungsbilanz des Gläubigerlandes einen Leistungsbilanzüberschuß auf bzw. die des Schuldnerlandes ein Defizit, besitzt letzteres einen Handelsbilanzsaldo, der geringer ist als jener der folgenden Periode. Das Schuldnerland kann in dieser Periode sogar ein Handelsbilanzdefizit besitzen. Schon zwei Perioden nach Auftreten des Budgetdefizits hat der Handelsbilanzsaldo sein neues, gegenüber dem alten langfristigen Gleichgewicht im Betrag reduziertes stationäres Niveau erreicht. Auch ist der Betrag des Handelsbilanzsaldos geringer als jener des Handelsbilanzsaldos der vorhergehenden Periode.

Ein staatliches Budgetdefizit im Schuldnerland erzeugt folgende Zahlungsbilanzdynamik: Ist der kurzfristige Zinssatz eine Periode nach Auftreten des Budgetdefizits mindestens so hoch wie im alten langfristigen Gleichgewicht, weist die Zahlungsbilanz des Schuldnerlandes in der Periode des Budgetdefizits ein Leistungsbilanzdefizit auf und die des Gläubigerlandes einen Überschuß. Im Fall, daß der kurzfristige Zinssatz auch eine Periode nach Auftreten des Budgetdefizits unter seinem alten langfristigen Niveau verharrt, läßt sich wieder 
keine Aussage über die Struktur der Leistungsbilanz treffen. Eine Periode nach Auftreten des Budgetdefizits weist die Zahlungsbilanz des Schuldnerlandes ein Leistungsbilanzdefizit auf und die des Gläubigerlandes einen Überschuß. Schon zwei Perioden nach Auftreten des Budgetdefizits ist die Leistungsbilanz wieder ausgeglichen.

Weist die Zahlungsbilanz des Schuldnerlandes in der Periode des Budgetdefizits ein Leistungsbilanzdefizit auf bzw. die des Gläubigerlandes einen Überschuß, besitzt das Schuldnerland in dieser Periode einen Saldo der Handelsbilanz, der geringer ist als der im neuen langfristigen Gleichgewicht (ein Handelsbilanzdefizit ist möglich). Weist die Zahlungsbilanz des Schuldnerlandes einen Leistungsbilanzüberschuß auf bzw. die des Gläubigerlandes ein Defizit, besitzt das Schuldnerland einen Handelsbilanzüberschuß, der höher ist als der Saldo seiner Handelsbilanz der folgenden Periode (dieser ist unbestimmt). Schon zwei Perioden nach Auftreten des Budgetdefizits hat der Handelsbilanzsaldo sein gegenüber dem alten langfristigen Gleichgewicht im Betrag erhöhtes stationäres Niveau erreicht. Der neue stationäre Handelsbilanzüberschuß des Schuldnerlandes ist hierbei höher als der Saldo seiner Handelsbilanz eine Periode nach Auftreten des Budgetdefizits.

\subsection{Fazit}

Wie die entwickelten Modelle und die skizzierten Ergebnisse aus der Literatur zeigen, können der Außenhandel und die Finanzierungsentscheidung des Staates auf verschiedenartige Weise die Entwicklung einer Volkswirtschaft beeinflussen. Es ist dabei zwischen den transitorischen und den permanenten Effekten zu unterscheiden, da beide durchaus gegenläufig sein können.

Eine Öffnung zum Weltmarkt kann sich für die Individuen wohlfahrtsmindernd auswirken. Unterschiedliche Generationen werden unterschiedlich betroffen, und ein Land als ganzes kann durch Freihandel schlechter gestellt werden. Die Aufnahme von Außenhandel setzt ökonomische Anpassungsprozesse in Kraft, die sich international unterscheiden. Kapitalakkumulationsraten verändern sich, 
nationale sowie internationale Einkommensumverteilungsprozesse werden initiiert, und die Zinsstruktur wird beeinflußt. In Übergangsgleichgewichten als normal erachtete Handels- und Leistungsbilanzungleichgewichte sind auch langfristig eher die Regel als die Ausnahme. Sie manifestieren den permanenten Güteraustausch zwischen den Ländern und die in einem Laissez-Faire-Regime eröffneten internationalen Investitionsmöglichkeiten.

Die Finanzierungsentscheidung des Staates ist für die Entwicklung einer Volkswirtschaft von entscheidender Bedeutung. Staatsverschuldung wirkt sich auf die Akkumulation von Produktivkapital, die Güterallokation und die Zinssätze aus. Sie erzeugt einen nationalen und internationalen Einkommensumverteilungsprozeß, der sich auf die Wohlfahrt einzelner Generationen unterschiedlich auswirkt. In einer geschlossenen Volkswirtschaft und bei großen offenen Volkswirtschaften wirkt die Staatsverschuldung reduzierend auf den Kapitalstock, und im zweiten Fall besitzt sie auch internationale Übertragungswirkungen. Die staatliche Anleiheemission wirkt auf die Zinsstruktur und auf die Güterallokation. Der mit einer positiven Staatsschuld verbundene Zinsendienst trifft mitunter noch nicht geborene Generationen, was eine intertemporale Steuerüberwälzung impliziert. Aufgrund der internationalen Übertragungswirkungen der Staatsverschuldung beeinflußt die nationale staatliche Finanzierungsentscheidung auch die Entwicklung anderer Länder.

Bei den durch Außenhandel und Staatsverschuldung induzierten Effekten lassen sich zwei Wirkungskanäle erkennen: Zum einen wird die Akkumulationsrate des Produktivkapitals beeinflußt und zum anderen der Kurswert der Vermögenstitel. Beide Wirkungskanäle manifestieren sich in einer Veränderung der Zinssätze. Die Zinssätze sind die intertemporalen Relativpreise, die für die Lebensplanung der Individuen determinierend sind. Die Veränderung der Akkumulationsrate des Produktivkapitals beeinflußt auf der Produktionsseite zum einen das Produktionsniveau und zum anderen die primäre Einkommensverteilung. Die Veränderung des Kurswertes der Vermögenstitel erzeugt einen Vermögenseffekt, der Umverteilungsprozesse induziert.

Die beiden in realen Volkswirtschaften simultan auftretenden Wirkungskanäle wurden aus analytischen Gründen separiert. Nur so war es möglich, die Wir- 
kungsmechanismen exakt zu eruieren. Es bestehen natürlich Wechselwirkungen zwischen der Veränderung der Akkumulationsrate des Produktivkapitals und der Veränderung des Kurswertes von Vermögenstiteln. Diese Wechselwirkungen lassen sich allerdings auf die beiden diskutierten Wirkungskanäle zurückführen und stellen keinen neuen Wirkungskanal dar. 
Michael Ganske - 978-3-631-75472-6

Downloaded from PubFactory at 01/11/2019 04:35:58AM

via free access 


\section{Anhang A: Das Blanchard-Modell eines kleinen Landes}

Das Modell von Blanchard (1985) zeichnet sich dadurch aus, daß die Akteure eine über ihre Lebenszeit konstante Sterbewahrscheinlichkeit besitzen. Das Modell ist in stetiger Zeit formuliert, und zu jedem Zeitpunkt wird eine neue Generation von Akteuren geboren. Die modellierte Ökonomie besitzt somit einen unendlichen Zeithorizont, nicht aber die Akteure, die in ihr leben. Im folgenden soll der Fall eines kleinen Landes betrachtet werden. Hierfür werden zuerst die Strukturmerkmale des Modells vorgestellt und dann die Modelldynamik diskutiert. Anschließend wird das Ricardo-Äquivalenztheorem diskutiert.

\section{A.1 Eigenschaften}

\section{Demographische Struktur}

Sei die Sterbewahrscheinlichkeit der Akteure durch die Sterberate $p$ parametrisiert. ${ }^{1}$ Die verbleibende Lebenszeit eines Akteurs ist somit eine Zufallsvariable und werde als $\chi$ definiert. Da die Sterberate als unabhängig vom Lebensalter angenommen wird, ist $\chi$ exponentialverteilt. ${ }^{2}$ Ihre Dichtefunktion ist also

${ }^{1} p$ wird gewöhnlich als Sterbewahrscheinlichkeit bezeichnet. Dies ist jedoch nicht ganz korrekt, da $p$ tatsächlich der Verteilungsparameter der die verbleibende Lebenszeit eines Akteurs beschreibende Dichtefunktion ist, und er alle Werte zwischen null und unendlich annehmen kann. $p$ ist genau genommen eine Rate pro Zeiteinheit. Im Fall einer Formulierung in diskreter Zeit, kann $p$ bedenkenlos als Sterbewahrscheinlichkeit bezeichnet werden, da $p$ hier zwischen null und eins liegt. Für eine in diskreter Zeit formulierte Version des Blanchard-Modells siehe z.B. Frenkel und Razin (1996), Kap. 9-10.

${ }^{2}$ Die Exponentialverteilung wird aus diesem Grund auch als Verteilung ohne Gedächtnis bezeichnet. Die bedingte Verteilung der verbleibenden Lebenszeit ist unabhängig von der bereits erreichten Lebenszeit. 


$$
f_{\chi}(t)=p \cdot \exp (-p \cdot t)
$$

Ein Akteur, der zum Zeitpunkt $j$ geboren wird, ist zum Zeitpunkt $t \geq j$ mit einer Wahrscheinlichkeit von $F_{\chi}(t-j)=[-\exp (-p \cdot t)]_{0}^{t-j}=1-\exp [-p \cdot(t-j)]$ tot und damit mit einer Wahrscheinlichkeit von $\exp [-p \cdot(t-j)]$ noch am Leben. Mittels partieller Integration, läßt sich die Lebenserwartung der Akteure aus (A.1) wie folgt berechnen:

$$
\begin{aligned}
E(\chi) & =\int_{0}^{\infty} t \cdot p \cdot \exp (-p \cdot t) d t \\
& =\left[t \cdot p \cdot-\frac{1}{p} \cdot \exp (-p \cdot t)\right]_{0}^{\infty}-\int_{0}^{\infty} p \cdot-\frac{1}{p} \cdot \exp (-p \cdot t) d t \\
& =[-t \cdot \exp (-p \cdot t)]_{0}^{\infty}-\left[\frac{1}{p} \cdot \exp (-p \cdot t)\right]_{0}^{\infty}=\frac{1}{p} .
\end{aligned}
$$

$1 / p$ ist somit die Lebenserwartung der Akteure und gleichzeitig ihre erwartete verbleibende Lebenszeit, unabhängig von ihrem Alter. ${ }^{1}$ Dies verdeutlicht die Problematik der Annahme einer vom Alter unabhängigen Sterbewahrscheinlichkeit. Interpretiert man (A.2) allerdings als Lebenserwartung einer Familie, also $p$ als die Rate, mit der eine Familie mangels Nachkommen ausstirbt, so wird die Annahme eines konstanten $p$ realistischer. Die Annahme eines konstanten $p$ ist, wie weiter unten gezeigt wird, von Nöten, will man bei der Herleitung der makroökonomischen Größen den Aggregationsprozeß vereinfachen. $p$ ist ein die marginale Konsumneigung determinierender Parameter. Diese ist damit unabhängig vom Alter, was eine einfache Aggregation der individuellen Konsumfunktionen in die gesellschaftliche Konsumfunktion ermöglicht.

Die Bevölkerungsgröße ist, anders als die verbleibende Lebenszeit eines einzelnen Akteurs, keine Zufallsvariable. Die Generationen verkleinern sich deterministisch mit der Rate $p$. Dies ist deshalb der Fall, da angenommen wird, daß die

${ }^{1} 1 / p$ ist der Zeithorizont über den die Akteure planen. Bei einer gegen null gehenden Sterberate, konvergiert der Lebenshorizont gegen unendlich, und man erhält die demographische Struktur des Weil-Modells, das in Weil (1989) entwickelt wird. 
Anzahl der zu jedem Zeitpunkt geborenen Akteure so groß ist, daß das Gesetz der großen Zahlen zur Wirkung kommt. ${ }^{1}$ Anders als in Blanchard (1985) wird unterstellt, daß die Bevölkerung mit einer konstanten Rate $n>0$ wächst. Die Bevölkerungsgröße zum Zeitpunkt $t$ beträgt $L(t)=\exp (n \cdot t)$. Damit $L$ mit einer Rate von $n$ wächst, muß die Größe der in $t$ geborenen Generation $(p+n) \cdot \exp (n \cdot t)$ betragen. In $t$ sterben $p \cdot \exp (n \cdot t)$ Akteure. Damit beträgt das Nettowachstum $d L / d t=n \cdot \exp (n \cdot t)=n \cdot L$, und die Wachstumsrate der Bevölkerung ist somit $(d L / d t) / L=n$.

\section{Erwartungsnutzenmaximierung}

Es wird angenommen, daß die Akteure ihren über die Lebenszeit erwarteten Nutzen maximieren. Sei $c(j, v)$ der Konsum eines zum Zeitpunkt $j$ geborenen Akteurs zum Zeitpunkt $v$. Es wird unterstellt, daß die Nutzenfunktion der Akteure logarithmisch ist, also gilt $u(c(j, v))=\log c(j, v)$. Die intertemporale Substitutionselastizität, zwischen dem Konsum zweier Zeitpunkte, wird durch den Kehrwert der relativen Veränderung des Grads der Steigung eine Indifferenzkurve, als Reaktion auf eine relative Veränderung des Konsummengenverhältnisses, gegeben. Einfacher ausgedrückt mißt die intertemporale Substitutionselastizität die Zinssensitivität der intertemporalen Konsumallokation. ${ }^{2}$ Sei die

${ }^{1}$ Sei $s$ die Anzahl der Akteure einer aus $m$ Akteuren bestehenden Generation, die zu einem Zeitpunkt sterben. Dann gilt:

$$
\lim _{m \rightarrow \infty} \operatorname{Pr}(|s / m-p| \geq \varepsilon)=0 \Leftrightarrow \lim _{m \rightarrow \infty} \operatorname{Pr}(|s / m-p|<\varepsilon)=1, \forall \varepsilon>0 .
$$

Bei einer hinreichend großen Population, ist die Rate, mit der eine Generation ausstirbt, faktisch deterministisch und entspricht der stochastischen individuellen Sterberate. Zum Gesetz der großen Zahlen, siehe z.B. Hogg und Craig (1978), S. 93.

${ }^{2}$ Die intertemporale Substitutionselastizität gibt an, wie leicht der Konsum über die Zeit substituierbar ist. Je niedriger sie ist, desto stärker verändert sich das Verhältnis der Grenznutzen, bei einer Veränderung des Konsummengenverhältnisses. Ökonomisch bedeutet dies, daß die Akteure um so weniger bereit sind von einem gleichförmigen Konsumpfad abzuweichen, je niedriger die intertemporale Substitutionselastizität ist. Je höher sie ist, desto eher sind die Akteure bereit, einen stark fluktuierenden Konsumpfad hinzunehmen, um schon geringe Dif- 
Substitutionselastizität als $\sigma$ definiert und der Konsum zum Zeitpunkt $t_{1} c\left(t_{1}\right)$ bzw. der Konsum zum Zeitpunkt $t_{2} c\left(t_{2}\right)$. Dann gilt:

$$
\sigma=\frac{\frac{d\left[c\left(t_{1}\right) / c\left(t_{2}\right)\right]}{c\left(t_{1}\right) / c\left(t_{2}\right)}}{\frac{d\left\{u^{\prime}\left[c\left(t_{1}\right)\right] / u^{\prime}\left[c\left(t_{2}\right)\right]\right\}}{-u^{\prime}\left[c\left(t_{1}\right)\right] / u^{\prime}\left[c\left(t_{2}\right)\right]}}
$$

Läßt man $t_{2}$ gegen $t_{1}$ gehen, erhält man

$$
\sigma(c)=-\frac{u^{\prime}(c)}{c \cdot u^{\prime \prime}(c)}
$$

Bei der angenommenen logarithmischen Nutzenfunktion gilt folglich $\sigma=1$. Damit ist die intertemporale Substitutionselastizität zwischen den Konsumnachfragen zweier Zeitpunkte 1. Der Vorteil der Annahme einer logarithmischen Nutzenfunktion liegt darin, daß hier die marginale Konsumneigung der Akteure nur vom tatsächlichen subjektiven Diskontfaktor abhängt und nicht vom Zinspfad. ${ }^{1} \mathrm{Da}$ die Akteure eine positive Zeitpräferenzrate $\rho$ besitzen, wird der zukünftige Konsum in der Gegenwart als weniger nutzenstiftend bewertet. ${ }^{2}$ Ausgehend vom Zeitpunkt $t$, maximieren die Akteure ihren Erwartungsnutzen

ferenzen zwischen der individuellen Zeitpräferenz und der Rendite der Vermögenstitel auszunutzen.

${ }^{1}$ Eine Erhöhung des Zinssatzes, bei gegebenem Vermögen, hat zwei Effekte. Der Konsum in der Zukunft wird im Verhältnis zum Konsum in der Gegenwart relativ günstiger, so daß sich die Nachfrage in die Zukunft verlagert (dies ist der intertemporale Substitutionseffekt ISE). Ein gestiegener Zinssatz bewirkt aber auch eine erhöhte Verzinsung der Vermögenstitel und damit ein erhöhtes Einkommen, was den gegenwärtigen und zukünttigen Konsum erhöht (dies ist der Einkommenseffekt EE). Je höher $\sigma$ ist, desto dominierender ist der ISE bzw. je niedriger $\sigma$ ist, desto dominierender ist der EE. Im Fall der logarithmischen Nutzenfunktion (hier gilt $\sigma=1$ ) heben sich der ISE und der EE auf, was die marginale Konsumneigung unabhängig vom Zinspfad macht.

${ }^{2}$ Siehe Epstein und Hynes (1983) zur Rolle der Zeitpräferenz in dynamischen Modellen. 


$$
E_{t} U=E_{t}\left[\int_{t}^{\infty} \log [c(j, v)] \cdot \exp [-\rho \cdot(v-t)] d v\right]
$$

Der multiplikative Diskontfaktor $\exp [-\rho \cdot(v-t)]$ spiegelt die positive Zeitpräferenz wider. Den Akteuren ist ihre Sterblichkeit bewußt, so daß sie ihre Sterbewahrscheinlichkeit in ihr Maximierungskalkül einbeziehen. Die Wahrscheinlichkeit, daß sie in $v \geq t$ am leben sind, wenn sie es in $t$ waren, beträgt gemäß (A1) $\exp [-p \cdot(v-t)]$. Damit wird aus (A.5):

$$
E_{t} U=\int_{t}^{\infty} \log [c(j, v)] \cdot \exp [-(\rho+p) \cdot(v-t)] d v
$$

Die positive Sterbewahrscheinlichkeit bedingt, daß der zukünftige Konsum unsicher ist. Als Folge wird er mit einem höheren Abschlag bewertet, als es die Zeitpräferenzrate $\rho$ bedingt. Die endliche Lebenszeit bewirkt, daß die tatsächliche Zeitpräferenzrate höher ist und der tatsächliche Diskontfaktor damit niedriger. Die tatsächliche Zeitpräferenzrate ist $(\rho+p)$ und der tatsächliche Diskontfaktor $\exp [-(\rho+p) \cdot(v-t)]$. Der zukünftige Nutzen wird noch stärker abdiskontiert. $\mathrm{Da}$ die Sterbewahrscheinlichkeit $p$ vom Alter unabhängig ist hat zur Folge, daß sich die zu maximierende Zielfunktion (A.6) über die Zeit nicht verändert. Somit tritt kein Problem der zeitlichen Inkonsistenz auf. Ein in der Vergangenheit gewählter optimaler Konsumpfad ist in der Gegenwart immer noch optimal.

Die Akteure beziehen ein Arbeitseinkommen. Da ihre Arbeitsproduktivität als unabhängig vom Alter angenommen wird, ist auch ihr Arbeitseinkommen über die Zeit konstant. Ein Akteur, der zum Zeitpunkt $j$ geboren wird, bezieht in allen $v \geq j$, so lange er am Leben ist, ein Arbeitseinkommen von $w(v)$. Die Annahme eines vom Alter unabhängigen Arbeitseinkommen vereinfacht die Herleitung des gesellschaftlichen Humanvermögens, welches das Aggregat der individuellen Humanvermögen der Gesellschaftsmitglieder ist. 


\section{Versicherungen}

Den Akteuren ist es möglich, ihren Konsumpfad vom Einkommenspfad zu trennen, indem sie sich verschulden oder einen Teil ihres Einkommens sparen. Da ihre verbleibende Lebenszeit allerdings eine Zufallsvariable ist, besteht Unsicherheit über den Zeitpunkt ihres Todes. Somit ist es möglich, daß sie verschuldet oder mit einem positiven Finanzvermögen sterben. Die Akteure sind Erwartungsnutzenmaximierer, und sie besitzen kein Vermächtnismotiv, wie z.B. in Barro (1974). Aus diesem Grund kann es für sie nicht optimal sein, mit einer positiven Vermögensposition zu sterben. Auch ist es plausibel anzunehmen, daß es ihnen verboten ist verschuldet zu sterben. So müssen sie ihren Erwartungsnutzen unter einer Nichtnegativitätsrestriktion ihres Finanzvermögens maximieren. Es besteht zwar individuelle Unsicherheit, aber aufgrund der Annahme einer großen Anzahl von Akteuren pro Generation, ist das aggregierte Verhalten deterministisch ( zu jedem Zeitpunkt $t$ sterben $p \cdot \exp (n \cdot t)$ Akteure). Dies ermöglicht die Bildung eines Marktes für Versicherungen. ${ }^{1}$

Für die Akteure ist es optimal Versicherungen folgender Ausgestaltung abzuschließen: Sie treten den Eigentumsanspruch an ihrem Finanzvermögen, für die Zeit nach ihrem Ableben, an die Versicherungsgesellschaft ab. Im Fall des Todes eines Akteurs, fällt sein gesamtes Finanzvermögen somit der Versicherungsgesellschaft zu. Als Gegenleistung erhält der Akteur von der Versicherung eine Prämie, die so lange zahlbar ist, wie er lebt. Faktisch schließt der Akteur somit eine private Rentenversicherung ab, wobei er ab der Beitragszahlung entsprechende Rentenbezüge erhält. Im Fall, daß ein Akteur einen Kredit aufnimmt, muß er, da er nicht verschuldet sterben darf, diesen Kredit durch eine Versicherung absichern. Im Fall seines Todes, tilgt die Versicherungsgesellschaft dann seine Schulden. Faktisch schließt der Akteur somit eine Lebensversicherung ab, wobei die Versicherungssumme seiner Verschuldung entspricht. Es stellt sich nun die Frage nach der Versicherungsprämie.

\footnotetext{
' Zur Rolle von Versicherungen bei unsicherer Lebenserwartung vgl. Yaari (1965), S.140f.
} 
Sei $r(t)$ die Rendite risikoloser Vermögenstitel. ${ }^{1}$ Sei ferner angenommen, daß der Versicherungsmarkt kompetitiv ist. Die Versicherungsgesellschaften erzielen somit keine Gewinne. Da die Sterberate pro Zeiteinheit $p$ beträgt, ist dies auch die Höhe der Versicherungsprämie. Die tatsächlichen Kreditkosten für die Akteure belaufen sich somit auf $r+p$ (= risikoloser Zinssatz + Versicherungsprämie). Das gleiche gilt für die tatsächliche Verzinsung ihres Vermögens. Aus der Sicht der Lebensversicherungsgesellschaft reicht die Prämie $p$ gerade aus, die Kredite der mit einer Rate von $p$ sterbenden Akteure zu tilgen. Das gleiche gilt für die an die Sparer fließende Prämie. Hier reicht das mit einer Rate von $p$ an die Rentenversicherungsgesellschaft fließende Vermögen der verstorbenen Anleger gerade aus, um die Prämie von $p$ zu zahlen. $p$ ist somit die faire Versicherungsprämie, da die Versicherungsgesellschaften bei ihr keine Gewinne erzielen können.

\section{Konsumfunktionen}

Sei $a(j, v)$ das Finanzvermögen eines in $j \leq v$ geborenen Akteurs, zum Zeitpunkt $v$. Dann lautet seine dynamische Budgetrestriktion:

$$
\frac{d a(j, v)}{d v}=[r(v)+p] \cdot a(j, v)+w(v)-c(j, v) \text {. }
$$

Die Veränderung des Finanzvermögens ergibt sich aus der Differenz zwischen der Summe aus der tatsächlichen Verzinsung der Rentenpapiere (bzw. den tatsächlichen Zinszahlungen, die ein verschuldeter Akteur zahlen muß, wenn $a$ also negativ ist) und dem Arbeitseinkommen, und dem Konsum des Akteurs.

Nun muß noch die weiter oben angesprochene Nichtnegativitätsrestriktion des Finanzvermögens formalisiert werden. Würde der Kapitalmarkt den Akteuren keine Restriktion auf ihren Verschuldungsgrad auferlegen, so würden sie sich

\footnotetext{
1 Aufgrund des fehlenden Vermächtnismotivs, sind die gehandelten Vermögenstitel Rentenpapiere und keine Nullcoupon-Anleihen (Zero-Bonds). Dem Halter eines solchen Rentenpapiers fließt ein über die Zeit konstanter Zahlungsstrom zu.
} 
aus ihrem Nutzenmaximierungskalkül heraus unbegrenzt verschulden. Es würde eine Kettenbriefsituation (auch Ponzi-Spiel genannt) resultieren. Ein Akteur könnte jederzeit einen Kredit in beliebiger Höhe aufnehmen und seinen Verschuldungsgrad in der Zukunft derart erhöhen, daß er aus der Neuverschuldung den Zinsendienst, in Höhe von $(r(t)+p) \cdot$ Kreditsumme, bestreitet. Dabei müßte er seinen Kredit nie tilgen, so daß seine Verschuldung mit der Rate $r(t)+p$ gegen unendlich wachsen würde. Da der aufgenommene Kredit tatsächlich nie getilgt würde, wäre der aus ihm in der Gegenwart finanzierte Konsum frei. Um ein solches Verhalten auszuschließen, wird gefordert, daß der Gegenwartswert des Finanzvermögens asymptotisch nicht negativ sein darf, das heißt:

$$
\begin{gathered}
\lim _{v \rightarrow \infty}\left\{a(j, v) \cdot \exp \left[-\int_{t}^{v}[r(s)+p] d s\right]\right\} \\
=\lim _{v \rightarrow \infty}\{a(j, v) \cdot \exp [-(\bar{r}(t, v)+p) \cdot(v-t)]\} \geq 0 \\
\operatorname{mit} \quad \bar{r}(t, v):=\frac{1}{v-t} \cdot \int_{t}^{v} r(s) d s
\end{gathered}
$$

Ungleichung (A.8) besagt, daß die Verschuldung eines Akteurs langfristig nur mit einer Rate wachsen darf, die niedriger als der tatsächliche Zinssatz $\bar{r}+p$ ist. Es darf also kein Ponzi-Spiel möglich sein. $\exp [-(\bar{r}(t, v)+p) \cdot(v-t)]$ ist hierbei der Gegenwartsfaktor, der die Vermögensposition zum Zeitpunkt $v \geq t$ auf eine äquivalente Vermögensposition zum Zeitpunkt $t$ abdiskontiert. Diese zeitliche Homogenisierung macht Vermögenspositionen verschiedener Zeitpunkte vergleichbar. $\mathrm{Zu}$ beachten ist, daß für die Bildung des Diskontfaktors der durchschnittliche Zinssatz $\bar{r}(t, v)$ verwendet wird. Die Bildung eines durchschnittlichen Zinssatzes ist im allgemeinen notwendig, da der Zinspfad in der Regel nicht konstant ist. ${ }^{1}$

${ }^{1}$ Da hier der Fall eines kleinen Landes betrachtet werden soll, ist die Bildung eines durchschnittlichen Zinssatzes eigentlich nicht notwendig. $r(t)$ wird auf dem Weltkapitalmarkt determiniert und als über die Zeit konstant angenommen. Er ist unabhängig vom Verhalten der betrachteten Ökonomie (Annahme des kleinen Landes). Aufgrund der Vollständigkeit wird hier der durchschnittliche Zinssatz verwendet. 
Die Akteure maximieren ihren durch (A.6) gegebenen Erwartungsnutzen unter den Nebenbedingungen (A.7) und (A.8). Um ihre Konsumfunktionen zu gewinnen, bildet man die Hameltonfunktion: ${ }^{1}$

$$
\begin{aligned}
H=\log [c(j, v)] \cdot \exp [-(\rho+p) \cdot(v-t)] \\
+\mu(v) \cdot\{[r(v)+p] \cdot a(j, v)+w(v)-c(j, v)\}
\end{aligned}
$$

Die Bedingungen erster Ordnung, für ein Maximum von $E_{t} U$, sind:

$$
\begin{aligned}
& \frac{\partial H}{\partial c(j, v)}=\frac{1}{c(j, v)} \cdot \exp [-(\rho+p) \cdot(v-t)]-\mu(v)=0, \\
& \frac{\partial H}{\partial a(j, v)}=\mu(v) \cdot[r(v)+p]=-\dot{\mu} .
\end{aligned}
$$

Die Transversalitätsbedingung lautet:

$$
\lim _{v \rightarrow \infty}[a(j, v) \cdot \mu(v)]=0 .
$$

Die Ableitung der Bedingung (A.10) nach der Zeit und anschließende Auflösung der ursprünglichen Bedingung nach $\mu(v)$ ergibt, nach einsetzen der Werte für $\mu(v)$ und $\dot{\mu}$ in Bedingung (A.11) und Umformung:

$$
\frac{d c(j, v)}{d v}=\dot{c}=[r(v)-\rho] \cdot c(j, v) .
$$

Der individuelle Konsum der Akteure nimmt im Zeitablauf genau dann zu (ab), wenn der risikolose Zinssatz höher (niedriger) als die Zeitpräferenzrate ist. Es fällt auf, daß die Sterberate $p$ in Gleichung (A.13) nicht mehr erscheint. Dies ist deshalb der Fall, da sich die endliche Lebenszeit sowohl im tatsächlichen

1 Zum Maximumprinzip siehe z.B. Takayama (1994), S.451ff, oder auch Chiang (1992), S.167ff. $H$ ist die in Gegenwartswerten ausgedrückte Hameltonfunktion. $c(j, v)$ ist hier die Kontrollvariable, $a(j, v)$ die Zustandsvariable und $\mu(v)$ die Kozustandsvariable. 
subjektiven Diskontfaktor $\exp [-(\rho+p) \cdot(v-t)]$, als auch beim tatsächlichen Zinssatz $[r(r)+p]$ auf gleiche Art widerspiegelt. Für die aus dem Optimierungsproze $ß$ folgenden Gleichgewichtsallokationen bedeutet dies, daß sie $\mathrm{Pa}-$ reto-effizient sind, denn die Grenzrate der Substitution im Konsum stimmt hier mit dem Diskontfaktor überein, mit dem risikolose Vermögenstitel bewertet werden. Trotz der Diskrepanz zwischen dem tatsächlichen Zinssatz, der für die individuelle Konsumplanung relevant ist, und dem risikolosen Marktzinssatz, der für die gesamte Gesellschaft von Bedeutung ist, entsteht keine makroökonomisch wohlfahrtsmindernde Verzerrung der intertemporalen Konsumallokation. ${ }^{1}$

Die Transversalitätsbedingung (A.12) besagt, daß der Wert des Finanzvermögens eines Akteurs asymptotisch null sein muß. Dies ist deshalb der Fall, da es für einen Akteur nicht optimal sein kann mit einer positiven Vermögensposition zu sterben, denn sein Nutzen würde steigen, würde er sein Vermögen vollständig liquidieren. Die Kozustandsvariable $\mu(v)$ läßt sich als Gegenwartsschattenpreis des Einkommens interpretieren. Er gibt den auf den Zeitpunkt $t$ abdiskontierten Wert an, den ein zum Zeitpunkt $v$ stattfindender Vermögenszuwachs bewirkt. $\mu(v)$ ist somit der in Gleichung (A.8) erscheinende Diskontfaktor $\exp [-(\bar{r}(t, v)+p) \cdot(v-t)]$ Somit ist die Restriktion (A.8) nicht arbiträr, sondern resultiert aus dem individuellen Optimierungskalkül der Akteure. Ein Gleichgewicht auf dem Kapitalmarkt erfordert somit, daß sich kein Akteur in Kettenbrief-Manier verschuldet, und (A.8) ist tatsächlich mit Gleichheitszeichen erfüllt.

\footnotetext{
${ }^{1}$ Wie weiter oben bei der Diskussion der demographischen Struktur deutlich wurde, ist zwar die individuelle Lebenszeit der einzelnen Akteure stochastisch, nicht aber die demographische Entwicklung der gesamten Ökonomie. Die Generationen verkleinem sich deterministisch mit der Rate $\rho$, es werden aber ständig neue Generationen geboren. Für die Gesellschaft ist also der risikolose Zinssatz $r$ relevant, da ihre Entwicklung deterministisch ist und sie mit Sicherheit bis in die Unendlichkeit existiert. Somit ist makroökonomisch der Zinssatz auf risikolose Vermögenstitel von Bedeutung (er determiniert die makroökonomisch relevante ökonomische Substitutionsrate), für die einzelnen Akteure allerdings der um die Versicherungsprämie erhöhte tatsächliche Zinssatz (er determiniert die für die individuelle intertemporale Konsumplanung relevante ökonomische Substitutionsrate, da die Individuen sich zu ihm am Kapitalmarkt verschulden bzw. ihre Ersparnis investieren können).
} 
Um die individuelle Konsumnachfrage der Akteure als Funktion ihres Vermögens darzustellen, ist es notwendig, die dynamische Budgetrestriktion (A.7) in eine intertemporale Budgetrestriktion umzuformen. Unter Verwendung des Diskontfaktors $\exp [-(\bar{r}(t, v)+p) \cdot(v-t)]$ läßt sich (A.7) in eine intertemporale Budgetrestriktion transformieren, die in allen $T \geq t$ erfüllt sein muß:

$$
\begin{gathered}
a(j, T) \cdot \exp [-(\bar{r}(t, T)+p) \cdot(T-t)]+\int_{t}^{T} c(j, v) \cdot \exp [-(\bar{r}(t, v)+p) \cdot(v-t)] d v \\
=a(j, t)+\int_{t}^{T} w(v) \cdot \exp [-(\bar{r}(t, v)+p) \cdot(v-t)] d v
\end{gathered}
$$

Aus der Transversalitätsbedingung (A.12) weiß man, daß die Restriktion (A.8) tatsächlich mit Gleichheitszeichen erfüllt wird. Somit verschwindet für $T \rightarrow \infty$ der erste Term auf der linken Seite von (A.14). Damit lautet die intertemporale Budgetrestriktion eines zum Zeitpunkt $j$ geborenen Akteurs:

$$
\begin{array}{r}
\int_{t}^{\infty} c(j, v) \cdot \exp [-(\bar{r}(t, v)+p) \cdot(v-t)] d v=a(j, t)+\widetilde{w}(t) \\
\text { mit } \widetilde{w}(t):=\int_{t}^{\infty} w(v) \cdot \exp [-(\bar{r}(t, v)+p) \cdot(v-t)] d v
\end{array}
$$

Die intertemporale Budgetrestriktion (A.15) besagt, daß der Gegenwartswert des Konsums eines Akteurs mit der Summe aus seinem gegenwärtigen Finanzvermögen und dem Gegenwartswert seines Arbeitseinkommen (hier mit $\widetilde{w}(t)$ definiert) übereinstimmen muß. ${ }^{1}$ Der Gegenwartswert des Arbeitseinkommens eines Akteurs ist sein individuelles Humanvermögen.

Integriert man (A.13) nach der Zeit, erhält man unter der Verwendung von $\bar{r}(t, v)$ :

${ }^{1} \mathrm{Da}$ die Budgetrestriktion mit Gleichheitszeichen erfüllt wird, resultiert aus der Tatsache, daß der Grenznutzen des Konsums, unabhängig von der konsumierten Gütermenge, immer positiv ist. 


$$
c(j, v)=c(j, t) \cdot \exp [(\bar{r}(t, v)-\rho) \cdot(v-t)] .
$$

(A.16) stellt einen Bezug zwischen dem Konsumniveau zum Zeitpunkt $t$ und dem zum Zeitpunkt $v \geq t$ her. Ist der durchschnittliche risikolose Zinssatz höher (niedriger) als die Zeitpräferenzrate, dann steigt (fällt) der Konsum im Zeitverlauf. Das Einsetzen von (A.16) in die intertemporale Budgetrestriktion (A.15) ergibt dann schließlich die individuelle Konsumfunktion der Akteure, als eine Funktion ihres Vermögens:

$$
c(j, t)=(\rho+p) \cdot[a(j, t)+\widetilde{w}(t)] .
$$

Der individuelle Konsum der Akteure hängt von ihrem Gesamtvermögen ab, welches sich aus ihrem Finanzvermögen $a(j, t)$ und ihrem Humanvermögen $\widetilde{w}(t)$ zusammensetzt. Ihre marginale Konsumneigung beträgt $(\rho+p)$. Sie ist aufgrund der Annahme einer logarithmischen Nutzenfunktion unabhängig vom Zinssatz, da sich der ISE und der EE hier gegenseitig aufheben. Auch ist sie über die Lebenszeit konstant, da sowohl die Sterbewahrscheinlichkeit als auch die Zeitpräferenzrate als unabhängig vom Alter angenommen werden.

Bisher wurden die individuellen Größen hergeleitet. Will man die Entwicklung des Landes analysieren, muß man die makroökonomischen Größen herleiten, welche das Aggregat der individuellen Größen sind. Da die Sterbewahrscheinlichkeit sowie das Arbeitseinkommen als unabhängig vom Lebensalter angenommen werden, ist der Aggregationsprozeß einfach. Zur Herleitung des nationalen Konsums zu einem Zeitpunkt werden die individuellen Konsummengen der zu diesem Zeitpunkt lebenden Akteure aggregiert. Eine in $j \leq t$ geborene Generation besitzt eine anfängliche Größe von $(p+n) \cdot \exp (n \cdot j)$ Akteuren. Der sich zum Zeitpunkt $t$ noch am Leben befindende Anteil der Generation $j$ ist $\exp [-p \cdot(t-j)]$. Somit ergibt sich der aggregierte Konsum zum Zeitpunkt $t$ durch Integration über alle Generationen:

$$
C(t)=\int_{-\infty}^{t} c(j, t) \cdot(p+n) \cdot \exp (n \cdot j) \cdot \exp [-p \cdot(t-j)] d j
$$


Das aggregierte Finanzvermögen erhält man durch das gleiche Vorgehen. Es beträgt

$$
A(t)=\int_{-\infty}^{t} a(j, t) \cdot(p+n) \cdot \exp (n \cdot j) \cdot \exp [-p \cdot(t-j)] d j
$$

Das Humanvermögen der Gesellschaft ist, da das Arbeitseinkommen vom Alter unabhängig ist, gleich dem Gegenwartswert des Arbeitseinkommens eines einzelnen Akteurs mal der Bevölkerungsgröße:

$$
\begin{aligned}
\widetilde{W}(t) & =\widetilde{w}(t) \cdot \exp (n \cdot t) \\
& =\exp (n \cdot t) \cdot \int_{t}^{\infty} w(v) \cdot \exp [-(\bar{r}(t, v)+p) \cdot(v-t)] d v .
\end{aligned}
$$

Wie man aus Gleichung (A.17) ersieht, ist die individuelle marginale Konsumneigung $(\rho+p)$. Da sie unabhängig vom Lebensalter ist, ist dies auch die aggregierte marginale Konsumneigung. Somit ist die gesellschaftliche Konsumfunktion

$$
C(t)=(\rho+p) \cdot[A(t)+\widetilde{W}(t)]
$$

Analog zur durch Gleichung (A.13) gegebenen Entwicklung des individuellen Konsums, läßt sich auch die zeitliche Entwicklung des aggregierten Konsumverhaltens berechnen. Die Veränderung des aggregierten Konsums hängt hierbei von der Veränderung des gesellschaftlichen Gesamtvermögens ab. Letzteres setzt sich aus dem nationalen Finanzvermögen und dem nationalen Humanvermögen zusammen. Es ist also notwendig die Veränderung beider Vermögenskomponenten zu berechnen. Die Veränderung des Finanzvermögens erhält man hierbei durch die Differentiation von Gleichung (A.19) nach der Zeit: 


$$
\begin{aligned}
\frac{d A(t)}{d t} & =(p+n) \cdot \exp (n \cdot t) \cdot a(t, t)-p \cdot A(t) \\
& +\int_{-\infty}^{t} \frac{d a(j, t)}{d t} \cdot(p+n) \cdot \exp (n \cdot j) \cdot \exp [-p \cdot(t-j)] d j
\end{aligned}
$$

Der erste Term ist das Finanzvermögen der zum Zeitpunkt $t$ geborenen Generation. Da kein Vermächtnismotiv existiert, gilt $a(t, t)=0$. Der erste Term ist somit null. Der zweite Term ist das Vermögen der Akteure, die in $t$ sterben. Der dritte Term beschreibt die Veränderung des Finanzvermögens aller anderen Akteure. Unter Verwendung der individuellen dynamischen Budgetrestriktion (A.7), läßt sich (A.22) wie folgt schreiben:

$$
\dot{A}=r(t) \cdot A(t)+w(t) \cdot \exp (n \cdot t)-C(t) .
$$

Die Veränderung des nationalen Finanzvermögens ergibt sich aus der Differenz zwischen der Summe aus der Verzinsung des Vermögensbestands und dem aggregierten Arbeitseinkommen ( = individuelles Arbeitseinkommen mal Bevölkerungsgröße), und dem Gesellschaftskonsum. Es fällt auf, daß anders als das individuelle Finanzvermögen, das aggregierte Finanzvermögen nur eine Rendite von $r(t)$ besitzt. Die Verzinsung des individuellen Finanzvermögens beträgt $r(t)+p . p$ ist allerdings nur die Versicherungsprämie (Risikoprämie), die sich makroökonomisch aufhebt. $p \cdot A(t)$ stellt nur einen innergesellschaftlichen Transfer dar. Es ist das Finanzvermögen, das von den zum Zeitpunkt $t$ sterbenden Akteuren über die Versicherungsprämie auf die noch lebenden Akteure übergeht. Bei diesem Prozeß stellt sich keine Veränderung des nationalen Finanzvermögens ein.

Die Veränderung des nationalen Humanvermögens erhält man durch Differentiation von Gleichung (A.20) nach der Zeit:

$$
\frac{d \tilde{W}(t)}{d t}=\dot{\widetilde{W}}=[r(t)+p+n] \cdot \tilde{W}(t)-w(t) \cdot \exp (n \cdot t)
$$


Der erste Term reflektiert die Tatsache, daß die einzelnen Akteure ihren Einkommensstrom mit der Rate $r(t)+p$ abdiskontieren. Die Bevölkerung wächst mit einer Rate von $n$, was die Akkumulation des Humanvermögens um diesen Faktor erhöht. Der zweite Term ist das aggregierte Arbeitseinkommen zum Zeitpunkt $\boldsymbol{t}$. Es vermindert das Wachstum des Humanvermögens, da es ein Zahlungsstrom darstellt, der aus ihm abfließt. Aus dem Vergleich von (A.23) mit (A.24) wird der fundamentale Unterschied zwischen dem nationalen Finanzvermögen und dem nationalen Humanvermögen ersichtlich. Stirbt ein Akteur, so hat das keine Auswirkungen auf das nationale Finanzvermögen. Das nationale Humanvermögen nimmt allerdings ab, da die Arbeitskraft des verstorbenen Akteurs aus der Gesellschaft verschwindet. Dieser Unterschied bewirkt, daß die Sterbewahrscheinlichkeit in die Akkumulationsrate des nationalen Humanvermögens einfließt, nicht aber in die des nationalen Finanzvermögens. Auch ist die Wachstumsrate der Bevölkerung für das Wachstum des nationalen Humanvermögens von Bedeutung, nicht aber für das Wachstum des nationalen Finanzvermögens. Die Akteure werden ohne Vermögenstitel geboren, besitzen aber ihre Arbeitskraft, aus der sie einen Einkommensstrom antizipieren.

Die Veränderung des gesellschaftlichen Konsums erhält man durch Differentiation von Gleichung (A.21) nach der Zeit:

$$
\frac{d C(t)}{d t}=\dot{C}=(\rho+p) \cdot[\dot{A}+\dot{\tilde{W}}] \text {. }
$$

Unter Verwendung von Gleichung (A.23) und (A.24) erhält man:

$$
\begin{aligned}
\dot{C}= & (\rho+p) \\
& \cdot\{\underbrace{r(t) \cdot A(t)+w(t) \cdot \exp (n \cdot t)-C(t)}_{\tilde{A}}+\underbrace{[r(t)+p+n] \cdot \widetilde{W}(t)-w(t) \cdot \exp (n \cdot t)}_{\tilde{W}}\}
\end{aligned}
$$

Nach Umformung und Verwendung von Gleichung (A.21) ergibt sich: 


$$
\begin{aligned}
\dot{C} & =[r(t)+p+n] \cdot[C(t)-(\rho+p) \cdot A(t)]+(\rho+p) \cdot[r(t) \cdot A(t)-C(t)] \\
& =[r(t)-\rho+n] \cdot C(t)-(p+n) \cdot(\rho+p) \cdot A(t) .
\end{aligned}
$$

Die Veränderung des gesellschaftlichen Konsums hängt zum einen von der Differenz zwischen dem risikolosen Zinssatz und der Zeitpräferenzrate der Akteure $a b$, aber natürlich auch von der Wachstumsrate der Bevölkerung. Der Zweite Term in Gleichung (A.27) gibt den Effekt der positiven Sterberate der Akteure auf die Veränderung des gesellschaftlichen Konsums an. Ein höheres $p$ wirkt in Richtung einer Reduktion des Wachstums des gesellschaftlichen Konsums (die verstorbenen Akteure konsumieren nicht mehr). Der Effekt der positiven Sterberate ist hierbei um so größer, je höher die Zeitpräferenzrate ist, da mit ihr die Konsumneigung der Akteure zunimmt.

Für die Analyse der Entwicklung wachsender Ökonomien ist es sinnvoll und üblich das Verhalten in Pro-Kopf-Größen auszudrücken. ${ }^{1}$ Zur Herleitung der Veränderung des Pro-Kopf-Konsums benötigt man die Pro-Kopf-Versionen von (A.23), (A.24), (A.21) und (A.25):

$$
\begin{aligned}
& \dot{a}=r(t) \cdot a(t)+w(t)-c(t)-n \cdot a(t), \\
& \dot{\widetilde{w}}=[r(t)+p] \cdot \widetilde{w}-w(t), \\
& c(t)=(\rho+p) \cdot[a(t)+\widetilde{w}(t)], \\
& \dot{c}=(\rho+p) \cdot(\dot{a}+\dot{\tilde{w}}) .
\end{aligned}
$$

$c(t)$ ist hierbei der nationale Pro-Kopf-Konsum, $a(t)$ ist das nationale Pro-KopfFinanzvermögen und $\widetilde{w}(t)$ das nationale Pro-Kopf-Humanvermögen (alle zum Zeitpunkt $t$ ). Bei der Veränderung des Pro-Kopf-Finanzvermögens fällt der

${ }^{1}$ Die Pro-Kopf-Größen sind die durch die Bevölkerungsgröße dividierten Aggregate. Anders als in den Modellen des Haupttextes, unterscheiden sich hier die Mengen pro Einwohner nicht von den Mengen pro Arbeiter, da die Akteure per Annahme über ihre gesamte Lebenszeit arbeiten. 
Term $n \cdot a(t)$ auf. Die Veränderung des Pro-Kopf-Finanzvermögens wird durch das Wachstum der Bevölkerung reduziert, da die Akteure ohne Finanzvermögen in die Ökonomie geboren werden. Bei der Veränderung des Pro-Kopf-Humanvermögens erscheint die Wachstumsrate der Bevölkerung, anders als bei der aggregierten Veränderung, nicht. Das durchschnittliche Verhalten, im Hinblick auf die Veränderung des Humanvermögens, ist unabhängig vom Wachstum der Bevölkerung, da die Akteure ausgestattet mit Arbeitskraft in die Ökonomie geboren werden und das individuelle Humanvermögen unabhängig vom Alter ist. Um die Veränderung des Pro-Kopf-Konsums zu erhalten, setzt man die Gleichungen (A.28) und (A.29) in Gleichung (A.31) ein:

$$
\dot{c}=[r(t)+p] \cdot\{\underbrace{r(t) \cdot a(t)+w(t)-c(t)-n \cdot a(t)}_{\tilde{a}}+\underbrace{[r(t)+\rho] \cdot \widetilde{w}-w(t)}_{\widetilde{w}}\} .
$$

Unter Verwendung von Gleichung (A.30), läßt sich Gleichung (A.32) umformen:

$$
\begin{aligned}
\dot{c} & =[r(t)+p] \cdot[c(t)-(\rho+p) \cdot a(t)]+(\rho+p) \cdot[r(t) \cdot a(t)-c(t)-n \cdot a(t)] \\
& =[r(t)-\rho] \cdot c(t)-(p+n) \cdot(\rho+p) \cdot a(t) .
\end{aligned}
$$

Der erste Term manifestiert die Tatsache, daß der individuelle Konsum, bei einem Zinssatz der höher als die Zeitpräferenzrate ist, über die Lebenszeit zunimmt (vgl. (A.13)). Der zweite Term läßt sich folgendermaßen interpretieren: $\rho+p$ ist die marginale Konsumneigung. Somit ist der mit einem Pro-KopfFinanzvermögen von $a(t)$ verbundene Pro-Kopf-Konsum $(\rho+p) \cdot a(t)$. Zum Zeitpunkt $t$ werden $(p+n) \cdot \exp (n \cdot t)$ Akteure geboren (die Bruttowachstumsrate der Bevölkerung beträgt $(p+n))$. Da sie ohne Finanzvermögen in die $\mathrm{Ge}-$ sellschaft eintreten, senkt sich der durchschnittliche Pro-Kopf-Konsum um $(p+n) \cdot(\rho+p) \cdot a(t)$. Entscheidend für die Entwicklung des Pro-Kopf-Konsums ist somit die Tatsache, daß ständig neue Akteure ohne Finanzvermögen geboren werden und nicht der Umstand, daß sie eine endliche Lebenszeit besitzen. ${ }^{1}$

${ }^{1}$ Dies zeigt auch das Modell von Weil (1989). Hier werden zu jedem Zeitpunkt neue Generationen geboren, deren Akteure eine unendliche Lebenszeit besitzen. Auch diese Modellstruk- 


\section{Produktion und Technologie}

Die verfügbare Technologie kann vermöge der Produktionsfunktion

$$
X=F(K, L)
$$

beschrieben werden. $X$ ist hierbei das Nationalprodukt, $K$ der Kapitalstock und $L$ das Arbeitskräftepotential. Es wird eine Ein-Sektoren-Technologie unterstellt. Das Produktionsergebnis kann hierbei entweder konsumiert oder in den Kapitalstock investiert werden. Die Produktionsfunktion ist von neoklassischem Typ. Das heißt $F$ weist konstante Skalenerträge in $K$ und $L$ auf und jeder Produktionsfaktor positive aber abnehmende Grenzprodukte. Aufgrund der Annahme konstanter Skalenerträge läßt sich (A.34) folgendermaßen umformen:

$$
X=L \cdot F(K / L, 1)=L \cdot f(k)
$$

$k:=K / L$ ist hierbei die Kapitalintensität. Sei $x:=Y / L$ die Pro-Kopf-Produktion. Damit gilt

$$
x=f(k) .
$$

Die Grenzproduktivitäten von Kapital und Arbeit sind

$$
\frac{\partial X}{\partial K}=f^{\prime}(k) \quad \text { und } \quad \frac{\partial X}{\partial L}=f(k)-k \cdot f^{\prime}(k) .
$$

tur erzeugt die für das OLG-Modell typischen Resultate (siehe weiter unten). Vergleicht man die durch Gleichung (A.13) gegebene Veränderung des individuellen Konsum mit der Veränderung des Pro-Kopf-Konsum, so wird ersichtlich, daß sie sich nur durch den das Pro-KopfFinanzvermögen enthaltenden Term unterscheiden. Entscheidend für den Unterschied zwischen der Veränderung von individuellem Konsum und der Veränderung des Pro-Kopf-Konsums ist somit tatsächlich der Umstand, daß neu geborene Akteure kein Finanzvermögen besitzen. Würde man ein operatives Vermächtnismotiv, wie z.B. bei Barro (1974), unterstellen, so würde ein Transfer von Vermögenstiteln zwischen den Generationen resultieren, welcher den Unterschied zwischen individuellem Verhalten und aggregiertem Verhalten aufheben würde. 
Sei $\delta \geq 0$ die Abschreibungsrate des Kapitalstocks und $\zeta$ der Mietpreis für eine Einheit Kapital. Da $r$ der Zinssatz auf risikolose Vermögenstitel ist, und beide Anlageformen vollkommene Substitute im Portfolio der Anleger sind, muß $\zeta=r+\delta$ gelten. Sei der Güterpreis auf 1 normiert, dann beträgt der Gewinn einer repräsentativen Unternehmung

$$
\Pi=F(K, L)-(r+\delta) \cdot K-w \cdot L .
$$

Es wird unterstellt, daß die Marktstruktur kompetitiv ist. Die Unternehmungen versuchen, bei gegebenen $r$ und $w$, ihren Gewinn $\Pi$ zu maximieren. Aus den Bedingungen erster Ordnung folgt:

$$
\begin{aligned}
& f^{\prime}(k)=r+\delta, \\
& f(k)-k \cdot f^{\prime}(k)=w .
\end{aligned}
$$

Die Unternehmungen wählen das Faktoreinsatzverhältnis derart, daß die Faktoren mit ihrem Grenzprodukt entlohnt werden. Die Entlohnung der Produktionsfaktoren entspricht dem Produktionsergebnis (Euler-Theorem). Die Unternehmungen erzielen somit keinen Gewinn, es gilt also $\Pi=0 .{ }^{1}$

\section{A.2 Gleichgewichtsdynamik}

Betrachtet wird der Fall eines kleinen Landes. ${ }^{2}$ Die Weltwirtschaft besteht aus vielen Ökonomien des unter A.1 beschriebenen Typs, und es herrscht ein Laissez-Faire-Regime (international freier Güter- und Kapitalverkehr). Anteile am Produktivkapital in den einzelnen Ländern seien in ihrer Anlagefunktion vollkommene Substitute (Annahme vollkommen integrierter Kapitalmärkte). Das Produktivkapital erzielt somit auf der ganzen Welt eine Rendite von $r$. Ri-

\footnotetext{
${ }^{1}$ Es wird also nur das Faktoreinsatzverhältnis und damit die Gesamtproduktion der Industrie determiniert, nicht aber die Produktionsmengen der einzelnen Unternehmungen.

${ }^{2}$ Ein kleines Land zeichnet sich dadurch aus, daß seine Entwicklung keinen Einfluß auf die auf dem Weltmarkt determinierten Preise und Zinssätze besitzt.
} 
sikolose Vermögenstitel und Anteile am Produktivkapital sind in den einzelnen Ländern weiterhin vollkommene Substitute im Portfolio der Anleger. $r$ ist somit der Weltzinssatz. Aufgrund der Annahme des kleinen Landes ist er unabhängig vom Verhalten der betrachteten Volkswirtschaft und wird als über die Zeit konstant angenommen.

Die Differenz zwischen dem nationalen Pro-Kopf-Finanzvermögen und der Kapitalintensität der Produktion ist das nationale Pro-Kopf-Nettoauslandsvermögen, auch nationale Pro-Kopf-Auslandsposition genannt:

$$
e:=a-k
$$

Die nationale Auslandsposition ist dann $L \cdot e=E$. Die Leistungsbilanz gibt die Veränderung der Auslandsposition über die Zeit an. Sei die Leistungsbilanz mit $V$ definiert. Dann gilt:

$$
V=\dot{E}=\dot{L} \cdot e+L \cdot \dot{e}
$$

Sei die Pro-Kopf-Leistungsbilanz mit $v$ definiert. Da die Bevölkerung mit einer Rate von $n$ wächst gilt:

$$
v=n \cdot e+\dot{e} .
$$

Da weltweit ein homogenes Gut produziert wird, ist der einzige Zweck von Außenhandel, daß die inländische Produktion von den inländischen Ausgaben für Konsum und Investitionen abweichen kann (Inländer können die ausländische Produktion kaufen und Ausländer die inländische). Es wird somit auf den intertemporalen Aspekt des Außenhandels abgestellt, da Außenhandel hier internationale Kreditvergabe und Verschuldung bedeutet.

Um die Veränderung des nationalen Pro-Kopf-Finanzvermögens zu bestimmen, setzt man Gleichung (A.40) in Gleichung (A.28) ein. Unter Verwendung von Gleichung (A.39), erhält man: 


$$
\begin{aligned}
\dot{a} & =r \cdot a+f(k)-k \cdot(r+\delta)-c-n \cdot a \\
& =f(k)-r \cdot(k-a)-\delta \cdot k-n \cdot a-c .
\end{aligned}
$$

Mit $f(k)=w+(r+\delta) \cdot k$ ergibt sich

$$
\dot{a}=w+(r-n) \cdot a-c .
$$

Die Veränderung des Pro-Kopf-Konsums wird nach wie vor durch Gleichung (A.33) gegeben. Das durch (A.45) und (A.33) gegebene Differentialgleichungssystem ist linear in $a$ und $c$.

Um die Stabilitätseigenschaft des Systems zu ermitteln, bildet man die charakteristische Gleichung:

$$
\operatorname{det}\left\{\left[\begin{array}{cc}
r-\rho & -(p+n) \cdot(\rho+p) \\
-1 & r-n
\end{array}\right]-\left[\begin{array}{ll}
\lambda & 0 \\
0 & \lambda
\end{array}\right]\right\}=0
$$

Die Eigenwerte sind

$$
\begin{aligned}
\lambda_{1,2} & =\frac{1}{2} \cdot\left\{2 \cdot r-\rho-n \pm \sqrt{(2 \cdot r-\rho-n)^{2}-4 \cdot[(r-\rho) \cdot(r-n)-(p+n) \cdot(\rho+p)]}\right\} \\
& =\frac{1}{2} \cdot[2 \cdot r-\rho-n \pm(\rho+n+2 \cdot p)] .
\end{aligned}
$$

Das heißt $\lambda_{1}=r+p>0$ und $\lambda_{2}=r-\rho-n-p$. Das System ist somit genau dann sattelpunktstabil, wenn $r-\rho<p+n$ erfüllt ist, anderenfalls ist es instabil. Dieses Ergebnis läßt sich auch ökonomisch interpretieren: Teilt man Gleichung (A.13) durch den Konsum, so erkennt man, daß die Veränderungsrate des individuellen Konsums durch die Differenz zwischen dem risikolosen Zinssatz und der Zeitpräferenzrate gegeben wird. Die Sterberate der Bevölkerung beträgt $p$ und ihre Wachstumsrate $n$. Wäre nun die Veränderungsrate des individuellen Konsums größer als die Summe aus Sterberate und Wachstumsrate, würde der 
Pro-Kopf-Konsum der Gesellschaft ständig wachsen. ${ }^{1}$ Im Folgenden wird nun davon ausgegangen, daß die Stabilitätsrestriktion $r-\rho<p+n$ erfüllt ist, und die Ökonomie somit ein wohl definiertes langfristiges Gleichgewicht besitzt. Die Gleichgewichtsdynamik läßt sich unter Zuhilfenahme eines Phasendiagramms graphisch analysieren.

Darstellung A.1 zeigt das die Gleichgewichtsdynamik der Ökonomie beschreibende Phasendiagramm. Es müssen zwei Fälle unterschieden werden. Der erste Fall ist der, daß der risikolose Zinssatz höher als die Zeitpräferenzrate ist und der zweite Fall der, daß der risikolose Zinssatz niedriger als die Zeitpräferenzrate ist. ${ }^{2}$ Auf der Abszisse ist das nationale Pro-Kopf-Finanzvermögen abgetragen und auf der Ordinate der nationale Pro-Kopf-Konsum. Die $\dot{a}=0$-Gerade gewinnt man aus Gleichung (A.45). Auf ihr liegen alle Kombinationen von ProKopf-Konsum und Pro-Kopf-Finanzvermögen, bei denen sich das Pro-KopfFinanzvermögen im Zeitablauf nicht verändert. Die $\dot{c}=0$-Gerade gewinnt man aus Gleichung (A.33). Auf ihr liegen alle Kombinationen von Pro-Kopf-Konsum und Pro-Kopf-Finanzvermögen, bei denen sich der Pro-Kopf-Konsum im Zeitablauf nicht verändert. Ein langfristiges Gleichgewicht zeichnet sich dadurch aus, daß der Pro-Kopf-Konsum und das Pro-Kopf-Finanzvermögen über die Zeit konstant sind. Der Schnittpunkte der $\dot{a}=0$ - Geraden mit der $\dot{c}=0$ Geraden beschreibt somit das langfristige Gleichgewichte des kleinen Landes.

\footnotetext{
' Eine positive Veränderungsrate des individuellen Konsums ist langfristig nur dann aufrechtzuerhalten, wenn diese Veränderung durch die Veränderung der Bevölkerungsstruktur kompensiert wird. Die mit einer Rate von $p$ sterbenden Akteure verlassen das ökonomische System. Diese Akteure verändern ihren Konsum mit einer Rate von -1 (von positivem Konsum auf keinen Konsum). Die Bevölkerung wächst mit einer Rate von $n$. Die langfristig mögliche Veränderungsrate des individuellen Konsums erhöht sich um diese Rate, da die Berechnung des Pro-Kopf-Konsums über die mit der Rate $n$ wachsende Bevölkerung erfolgt.

${ }^{2}$ Wie aus Gleichung (A.13) bekannt ist, nimmt der individuelle Konsum im ersten Fall mit zunehmendem Lebensalter zu und sinkt im zweiten Fall mit zunehmenden Lebensalter.
} 
Fall 1: $\mathbf{r}>\rho$

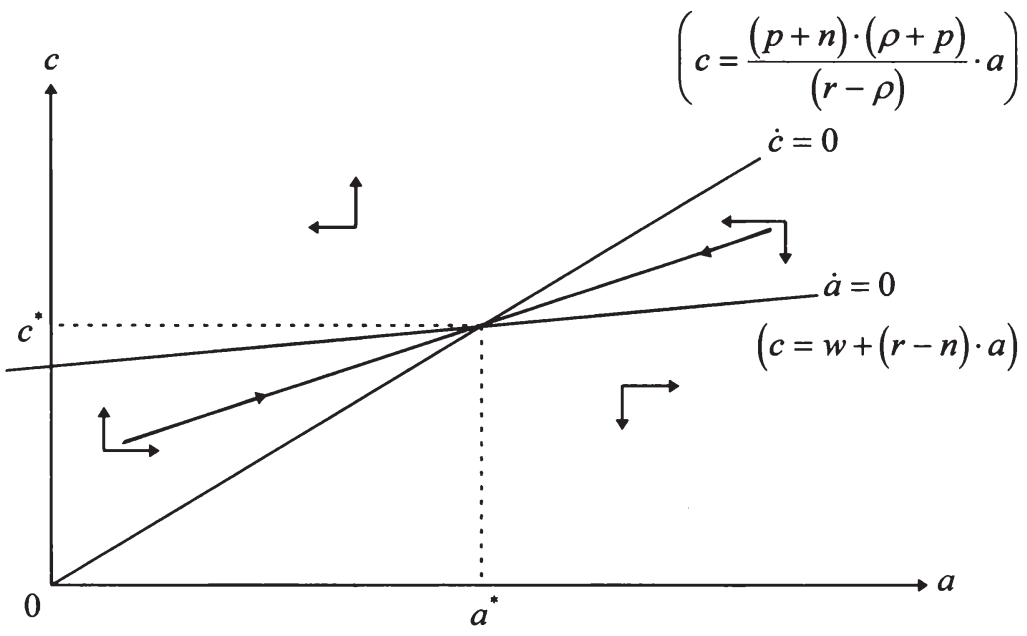

Fall 2: $r<\rho$

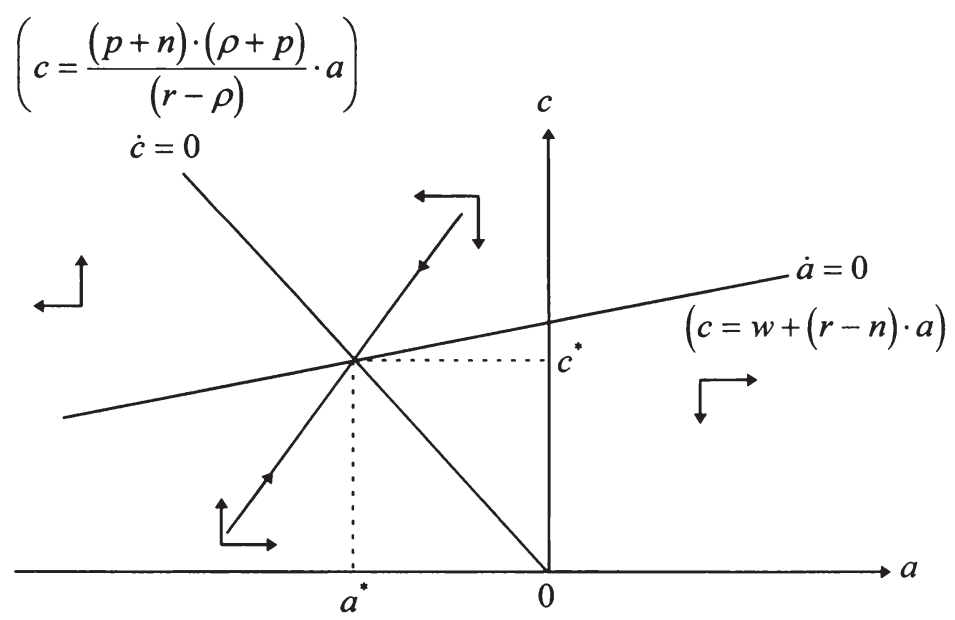

Darst. A.1: Gleichgewichtsdynamik 
Die $\dot{a}=0$-Gerade besitzt eine Steigung von $r-n$ und schneidet die Ordinate bei einem Wert, der dem Arbeitseinkommen entspricht. ${ }^{1}$ Die $\dot{c}=0$-Gerade geht durch den Ursprung, und das Vorzeichen ihrer Steigung entspricht dem Vorzeichen von $r-\rho$. Aus (A.45) und (A.33) folgt:

$$
\begin{aligned}
& \dot{a} \geq 0 \quad \Leftrightarrow \quad c \leq w+(r-n) \cdot a, \\
& \dot{c} \geq 0 \quad \Leftrightarrow \quad c \geq \frac{(p+n) \cdot(\rho+p)}{(r-\rho)} \cdot a .
\end{aligned}
$$

Die durch (A.48) und (A.49) beschriebenen dynamischen Kräfte werden in Darstellung A.1 durch Pfeile repräsentiert. Aufgrund der Sattelpunkteigenschaft gibt es genau einen Pfad (der stabile Ast), der hin zum langfristigen Gleichgewicht führt. Für gegebene Pro-Kopf-Finanzvermögen wird der Pro-KopfKonsum, durch die Forderung, daß sich die Ökonomie auf dem stabilen Ast befindet, eindeutig determiniert. Im Fall, daß $r>\rho$ gilt, nimmt der individuelle Konsum über die Lebenszeit zu, und die Akteure akkumulieren ein Finanzvermögen. Die Stabilitätsrestriktion bedingt, daß die Steigung der $\dot{c}=0$-Geraden größer als die der $\dot{a}=0$-Geraden ist. Im langfristigen Gleichgewicht $\left(c^{*}, a^{*}\right)$ sind der Pro-Kopf-Konsum und das Pro-Kopf-Finanzvermögen positiv. Im Fall, daß $r<\rho$ gilt, ist die Steigung der $\dot{c}=0-$ Geraden negativ. Hier ist das langfristige Gleichgewicht zwar auch mit einem positiven Pro-Kopf-Konsum verbunden, das Pro-Kopf-Finanzvermögen ist allerdings negativ. Der individuelle Konsum der Akteure nimmt über ihre Lebenszeit ab, und sie verschulden sich.

${ }^{1}$ Der Zinssatz ist größer als die Wachstumsrate der Bevölkerung $(r>n)$. Somit ist der Gleichgewichtspfad dynamisch effizient. Es ist bekannt, daß das langfristige Gleichgewicht einer OLG-Ökonomie vom Diamond-Typ dynamisch ineffizient sein kein (hier ist der Zinssatz niedriger als die Wachstumsrate der Bevölkerung, es herrscht also Überkapitalisierung); siehe z.B. Romer (1996), S. $81 \mathrm{ff}$. Die dynamische Ineffizienz wird im Diamond-Modell durch das über den individuellen Lebenszyklus drastisch abnehmende Arbeitseinkommen erzeugt (die Akteure beziehen nur in der Jugend ein Arbeitseinkommen). Im vorliegenden Modell ist das Arbeitseinkommen per Annahme vom Lebensalter unabhängig und somit über den individuellen Lebenszyklus konstant. Die Möglichkeit dynamischer Ineffizienz ist hier nicht gegeben. 
Die Ökonomie ist genau dann ein Gläubigerland (Schuldnerland), wenn ihr ProKopf-Finanzvermögen größer (kleiner) als die Kapitalintensität der Produktion ist. Im Fall $r<\rho$ ist die Ökonomie ein Schuldnerland. Im Fall $r>\rho$ ist sie nur dann ein Schuldnerland, wenn $k>a$ gilt. Man erkennt, daß je ungeduldiger die Akteure der Ökonomie sind, dies spiegelt sich in einer hohen Zeitpräferenzrate wieder, desto eher es sich um ein Schuldnerland handelt. Der Weltzins $r$ resultiert aus der Bedingung (A.39). Mit jedem Zinssatz $r$ korrespondiert eine optimale Kapitalintensität $k$. Das langfristige Gleichgewicht der Weltwirtschaft wird bei dem Zinssatz erreicht, bei dem das globale Pro-Kopf-Finanzvermögen gleich der globalen Kapitalintensität ist. ${ }^{1}$

Aus der Analyse des Phasendiagramms wird ersichtlich:

- Eine Erhöhung von $r$ erhöht das langfristige Pro-Kopf-Finanzvermögen der Gesellschaft bzw. senkt ihre langfristige Pro-Kopf-Verschuldung. Der langfristige Pro-Kopf-Konsum steigt, da der nationale Vermögensbestand gestiegen ist, und er höher verzinst wird. Im Fall eines negativen Pro-Kopf-Finanzvermögens sinkt oder steigt der Pro-Kopf-Konsum je nach dem, ob der negative intertemporale Terms of Trade Effekt (die Schulden müssen höher verzinst werden) die Auswirkung der reduzierten Pro-Kopf-Verschuldung überkompensiert oder nicht.

- Eine Erhöhung von $p$ reduziert den Betrag des langfristigen Pro-Kopf-Finanzvermögens. Eine kürzere individuelle Lebenszeit der Akteure reduziert somit die nationale Akkumulation von Finanzvermögenspositionen. Bei positivem Pro-Kopf-Finanzvermögen reduziert sich der langfristige Pro-KopfKonsum und bei negativem Pro-Kopf-Finanzvermögen erhöht er sich.

- Einer Erhöhung von $\rho$ reduziert das langfristige Pro-Kopf-Finanzvermögen der Gesellschaft und damit auch ihren langfristigen Pro-Kopf-Konsum. Je höher die Zeitpräferenzrate der Akteure eines Landes ist, desto weniger sind sie bereit in der Jugend Konsumverzicht zu üben. Dies wirkt sich negativ auf das gesellschaftliche Finanzvermögen aus.

${ }^{1}$ Global betrachtet kann die Pro-Kopf-Verschuldung nicht positiv sein. Zu jedem Kreditnehmer gibt es ein Kreditgeber, und der Kapitalstock muß jemandem gehören. 
- Eine Erhöhung von $n$ reduziert den Betrag des langfristigen Pro-Kopf-Finanzvermögens der Gesellschaft. Bei einem positiven Pro-Kopf-Finanzvermögen reduziert sich der langfristige Pro-Kopf-Konsum, bei einem negativen Pro-Kopf-Finanzvermögen erhöht er sich. Eine Erhöhung der Wachstumsrate der Bevölkerung bewirkt somit die qualitativ gleichen Effekte wie eine Erhöhung ihrer Sterberate. ${ }^{1}$

\section{A.3 Das Ricardo-Äquivalenztheorem}

Das Ricardo-Äquivalenztheorem besagt, daß die Gleichgewichtsallokation nicht davon beeinflußt wird, ob der Staat seine Ausgaben über Steuern oder durch Verschuldung finanziert. Der Pfad der Besteuerung und der staatlichen Budgetsalden hat also keine realen Effekte. Die Voraussetzungen für die Gültigkeit des Äquivalenztheorems sind: (i) Die Ökonomie wird von einem repräsentativen Akteur mit unendlicher Lebenszeit bevölkert; (ii) vollkommene Kapitalmärkte; (iii) die Besteuerung erfolgt über Pauschalsteuern; (iv) die zukünftige Steuerlast wird vom Akteur antizipiert; (v) der Pfad der Staatsausgaben ist gegeben. ${ }^{2}$

Anhand des Blanchard-Modells wird nun gezeigt, warum bei Verletzung der Bedingung (i) das Ricardo-Äquivalenztheorem nicht gilt. Hierfür wird die Existenz eines öffentlichen Sektors angenommen. Der Staat konsumiert Güter und finanziert diesen Konsum durch die Erhebung von Pauschalsteuern auf das Arbeitseinkommen der Akteure und durch die Emission von Staatsanleihen. Da die Finanzierungsentscheidung des Staates im Mittelpunkt der Analyse steht, wird angenommen, daß der Staatskonsum keinen Einfluß auf den Grenznutzen des privaten Konsums besitzt. Die Höhe des Staatskonsums hat somit keinen Effekt auf die Konsum- und Sparentscheidung der Akteure.

\footnotetext{
${ }^{1}$ Dies war zu erwarten. $n$ und $p$ determinieren das Bruttowachstum der Bevölkerung (die Bruttowachstumsrate ist $n+p$ ). Wie weiter oben gezeigt wurde ist der Umstand, daß in jedem Moment neue Akteure ohne Finanzvermögen in die Gesellschaft geboren werden, für die Modelldynamik von entscheidender Bedeutung.

${ }^{2}$ Vgl. Turnovsky (1995), S. 243f.
} 
Sei $C^{g}(t)$ der Staatskonsum zum Zeitpunkt $t, T(t)$ die zum Zeitpunkt $t$ erhobene Pauschalsteuer auf das Arbeitseinkommen der Akteure und $G(t)$ die Staatsverschuldung zum Zeitpunkt $t$. Die dynamische staatliche Budgetrestriktion lautet dann:

$$
\underbrace{\frac{d G(t)}{d t}}_{\text {Budgetdefizit }}=\underbrace{r(t) \cdot G(t)}_{\text {Zinsendienst }}+\underbrace{C^{g}(t)-T(t)}_{\begin{array}{c}
\text { primares } \\
\text { Budgetdefizit }
\end{array}} .
$$

Das primäre Budgetdefizit ist gleich der positiven Differenz zwischen Staatskonsum und Steuereinnahmen. Der Staat muß in jedem Augenblick Zinsen auf die von ihm emittierten Anleihen zahlen (Zinsendienst). Der Staat kann sich zum risikolosen Zinssatz $r$ am Kapitalmarkt verschulden, da er mit Sicherheit bis in die Unendlichkeit weiter existiert. Vergleicht man die Budgetrestriktion (A.50) mit der individuellen Budgetrestriktion der Akteure (A.7), so erkennt man, daß sich der für die einzelnen Akteure relevante Zinssatz von dem für den Staat relevanten Zinssatz durch die Sterberate $p$ unterscheidet.

Auch die Verschuldungsrate des Staates kann nicht unbegrenzt sein. Tritt der Staat am Kapitalmarkt als Schuldner auf, so wird auch ihm eine Verschuldung in Kettenbriefmanier untersagt. Analog zu der für die Akteure relevanten Bedingung (A.8), muß der Staat folgender Transversalitätsbedingung erfüllen:

$$
\begin{gathered}
\lim _{v \rightarrow \infty}\left\{G(v) \cdot \exp \left[-\int_{t}^{v} r(s) d s\right]\right\}=\lim _{v \rightarrow \infty}\{G(v) \cdot \exp [-\bar{r}(t, v) \cdot(v-t)]\}=0, \\
\text { mit } \quad \bar{r}(t, v)=\frac{1}{v-t} \cdot \int_{t}^{v} r(s) d s .
\end{gathered}
$$

Bedingung (A.51) besagt, daß die Staatsverschuldung langfristig nur mit einer Rate wachsen darf, die kleiner als der risikolose Zinssatz ist. Es darf also kein Ponzi-Spiel möglich sein. $\exp [-\bar{r}(t, v) \cdot(v-t)]$ ist hierbei der Gegenwartsfaktor, der die Verschuldung zum Zeitpunkt $v \geq t$ auf eine äquivalente Verschuldung zum Zeitpunkt $t$ abdiskontiert. Diese zeitliche Homogenisierung macht Verschuldungsgrade verschiedener Zeitpunkte vergleichbar. Man erkennt, daß 
sich der staatliche Gegenwartsfaktor vom individuellen Gegenwartsfaktor durch die Sterberate $p$ unterscheidet. Der für den Staat relevante Diskontfaktor läßt sich am besten als sozialer Diskontfaktor bezeichnen (im Gegensatz zum für die einzelnen Akteure relevanten individuellen Diskontfaktor). Dieser Unterschied zwischen der individuellen und der sozialen Diskontierungsrate führt, wie weiter unten gezeigt wird, zur Nichtneutralität einer intertemporalen Reallokation der Besteuerung.

Unter Verwendung des sozialen Diskontfaktors $\exp [-\bar{r}(t, v) \cdot(v-t)]$, läßt sich (A50) in eine intertemporale Budgetrestriktion, die in allen $T \geq t$ erfüllt sein muß, transformieren:

$$
\begin{aligned}
G(t)+ & \int_{t}^{T} C^{g}(v) \cdot \exp [-\bar{r}(t, v) \cdot(v-t)] d v \\
& =G(T) \cdot \exp [-\bar{r}(t, T) \cdot(T-t)]+\int_{t}^{T} T(v) \cdot \exp [-\bar{r}(t, v) \cdot(v-t)] d v
\end{aligned}
$$

Gemäß (A.51) verschwindet für $T \rightarrow \infty$ der erste Term auf der rechten Seite. Die intertemporale Budgetrestriktion des Staates lautet somit:

$$
G(t)=-\int_{t}^{\infty}\left[C^{g}(v)-T(v)\right] \cdot \exp [-\bar{r}(t, v) \cdot(v-t)] d v
$$

Budgetrestriktion (A.53) besagt, daß die gegenwärtige Staatsverschuldung gleich dem Gegenwartswert der zukünftigen primären Budgetüberschüsse sein muß. Ist der Staat gegenwärtig ein Nettoschuldner, dann muß sein Budget irgendwann in der Zukunft primäre Überschüsse aufweisen. Die Budgetrestriktion erfordert nicht, daß die Schulden irgendwann in der Zukunft getilgt werden oder, daß sie über die Zeit konstant sein müssen. (A.53) erfordert nur, daß die Wachstumsrate der Verschuldung geringer als der risikolose Zinssatz ist. Somit reduziert sich die Restriktion auf die Transversalitätsbedingung (A.51).

Die Besteuerung des Arbeitseinkommens der Akteure wirkt sich auf ihr Humanvermögen aus. Das Humanvermögen der Gesellschaft zum Zeitpunkt $t$ ist 


$$
\begin{aligned}
\widetilde{W}(t)=\exp (n \cdot t) \cdot \int_{t}^{\infty} w(v) \cdot \exp [-(\bar{r}(t, v)+p) \cdot(v-t)] d v \\
-\int_{t}^{\infty} T(v) \cdot \exp [-(\bar{r}(t, v)+p) \cdot(v-t)] d v
\end{aligned}
$$

Vergleicht man (A.54) mit (A.20) erkennt man, daß sich das gesellschaftliche Humanvermögen um den Gegenwartswert der gegenwärtigen und zukünftigen Besteuerung des Arbeitseinkommens reduziert. Die zeitliche Homogenisierung des Besteuerungspfades erfolgt bei der Ermittlung des Humanvermögens mittels des individuellen Diskontfaktors $\exp [-(\bar{r}(t, v)+p) \cdot(v-t)]$ und nicht mittels des sozialen Diskontfaktors. Der Grund ist folgender: Das gesellschaftliche Humanvermögen ist das Aggregat der individuellen Humanvermögen der Akteure. Für die Akteure ist, bei der zeitlichen Homogenisierung von erwarteten Vermögenspositionen, der um die Sterberate $p$ erhöhte tatsächliche Zinssatz relevant. $r+p$ ist die Rate, mit dem ihr Vermögen (ihre Schulden) verzinst wird (werden).

Um die Auswirkungen einer intertemporalen Reallokation der Steuern herzuleiten, sei folgende Politik betrachtet: Der Staat reduziert, bei gegebenem Konsumpfad, die Steuern zum Zeitpunkt $t$ und erhöht sie zum Zeitpunkt $t+v$ $(v>0)$. Aus der staatlichen Budgetrestriktion (A.53) folgt:

$$
\begin{aligned}
d T(t+v)= & -d T(t) \cdot \exp [\bar{r}(t, t+v) \cdot v], \\
& \text { mit } \quad \bar{r}(t, t+v)=\frac{1}{v} \cdot \int_{t}^{t+v} r(s) d s .
\end{aligned}
$$

Die Erhöhung der Steuer zum Zeitpunkt $t+v$ muß gleich dem Betrag der mit dem Zinsfaktor $\exp [\bar{r}(t, t+v) \cdot v]$ multiplizierten Steuerreduktion des Zeitpunkts $t$ sein. Die Steuerreduktion kann, bei gegebenen Konsumausgaben, nur durch eine Erhöhung des Schuldenstandes erreicht werden, und dieser wird mit $\bar{r}$ verzinst. Die Erhöhung der Steuer in $t+v$ reduziert den Schuldenstand auf das bis zum Zeitpunkt $t$ erreichte Niveau. 
Gemäß (A.54) ist der Effekt der intertemporalen Reallokation der Steuern auf das gesellschaftliche Humanvermögen:

$$
d \tilde{W}(t)=-d T(t)-d T(t+v) \cdot \exp [-(\bar{r}(t, t+v)+p) \cdot v]
$$

Unter Verwendung von (A.55) wird daraus:

$$
d \tilde{W}(t)=-d T(t) \cdot[1-\exp (-p \cdot v)]
$$

Da $d T(t)<0$ (Steuerreduktion in $t$ ), erhöht die Politik das gesellschaftliche Humanvermögen. Gemäß (A.21) erhöht sich damit auch der aggregierte Konsum. Aus (A.57) erkennt man, daß der Effekt um so ausgeprägter ist, je weiter in der Zukunft die kompensierende Steuererhöhung stattfindet und je höher die Sterbewahrscheinlichkeit ist. ${ }^{1}$ Die Wahrscheinlichkeit, daß ein zum Zeitpunkt $t$ lebender Akteur zum Zeitpunkt $t+v$ tot ist, beträgt $[1-\exp (-p \cdot v)]$. Dies ist auch die Wahrscheinlichkeit, daß ein durch die Steuerreduktion begünstigter Akteur nicht durch die in der Zukunft stattfindende Steuererhöhung belastet wird. Somit besitzt die intertemporale Steuerreallokation einen intergenerationalen Umverteilungseffekt und damit einen realen Einfluß auf das Vermögen und das Konsumverhalten der Akteure. Das Ricardo-Äquivalenztheorem gilt also nicht.

Ist die Lebenszeit der Akteure unendlich und $p$ somit null, besitzt die intertemporale Reallokation der Steuern keinen Einfluß auf das gesellschaftliche $\mathrm{Hu}-$ manvermögen, da der Ausdruck (A.57) dann null ist. Das heißt aber nicht, daß das Ricardo-Äquivalenztheorem dann gilt. Das aggregierte Konsumverhalten bleibt zwar von der Politikmaßnahme unberührt, nicht aber das individuelle Konsumverhalten. Gilt $p=0$, wird das Modell von seiner demographischen Struktur her zum Weil-Modell. Entscheidend für die ökonomische Relevanz der intertemporalen Steuerreallokation ist, daß die von der Politikmaßnahme Begünstigten die zukünftigen Belastungen nur teilweise tragen müssen. ${ }^{2}$ Die

\footnotetext{
${ }^{1}$ Zum Einfluß unsicherer Lebenserwartungen auf die Wirksamkeit intertemporaler Steuerverlagerungen siehe auch Wellisch (1991), Kap. 1.

${ }^{2}$ Vgl. Weil (1989), S. 193ff.
} 
Überwälzung der zukünftigen Steuerlast auf von der gegenwärtigen Steuerreduktion nicht begünstigten Akteure entsteht zum einen durch eine positive Sterbewahrscheinlichkeit, was (A.57) manifestiert, aber auch durch die Tatsache, daß ständig neue Generationen geboren werden. Die positive Sterbewahrscheinlichkeit verstärkt nur den Überwälzungsprozeß.

Um die Nichtgültigkeit des Ricardo-Äquivalenztheorems auch im Fall $p=0 \mathrm{zu}$ zeigen, sei angenommen, daß der Staat nur Umverteilungspolitik betreibt. Die in Pro-Kopf-Größen ausgedrückte dynamische staatliche Budgetrestriktion lautet dann:

$$
\dot{g}=r(t) \cdot g(t)-\tau(t)-n \cdot g(t)
$$

$g(t)$ ist die Pro-Kopf-Staatsverschuldung zum Zeitpunkt $t$ und $\tau(t)$ die ProKopf-Pauschalsteuer auf das Arbeitseinkommen der Akteure. $n$ ist wie gehabt die Wachstumsrate der Bevölkerung und wegen $p=0$ auch die Rate, mit der neue Akteure in die Ökonomie geboren werden. ${ }^{1}$ Im langfristigen Gleichgewicht gilt $r \cdot g-\tau-n \cdot g=0$ und damit:

$$
g-\frac{\tau}{r}=\frac{n \cdot g}{r}
$$

$\tau / r$ ist der Gegenwartswert der individuellen Steuerbelastung (die kapitalisierte Lebenssteuerlast). Die Differenz zwischen der Pro-Kopf-Staatsverschuldung und dem Gegenwartswert der von den einzelnen Akteuren in ihrem Leben zu zahlenden Steuern ist bei einer wachsenden Bevölkerung positiv. Emittierte Staatsanleihen stellen somit ein gesellschaftliches Nettovermögen dar, und die Staatsverschuldung besitzt Allokationswirkungen.

Die ökonomische Relevanz der Staatsverschuldung resultiert letztendlich aus der Tatsache, daß die durch den Zinsendienst bedingte Steuerlast auch von Akteuren getragen wird, die noch nicht am Leben sind und deren Konsum gegen-

${ }^{1}$ Bei $p>0$ und einer Wachstumsrate der Bevölkerung von $n$, ist die Rate, mit der neue Akteure in die Ökonomie geboren werden, $p+n$. 
wärtig nicht bewertet wird. So hat das Ricardo-Äquivalenztheorem in Barro (1974) Gültigkeit, da dort die einzelnen Generationen durch ein Vermächtnismotiv verbunden sind. Bei Barro wird der Nutzen einer Generation durch den Nutzen der ihr folgenden Generation positiv beeinflußt. ${ }^{1}$ Das Vermächtnismotiv bedingt, daß der Konsum zukünftiger Generationen in der Gegenwart bewertet wird und damit auch die Steuerlast zukünftiger Generationen. Die emittierten Staatsanleihen stellen dann kein gesellschaftliches Nettovermögen mehr dar, da die mit ihrem Zinsendienst verbundene zukünftige Besteuerung vollständig in das Bewertungskalkül der in der Gegenwart lebenden Akteure einfließt. ${ }^{2}$

${ }^{1}$ Vgl. Barro (1974), S. 1100.

${ }^{2}$ Die Gültigkeit des Ricardo-Äquivalenztheorems wird aber nur dann hergestellt, wenn das Vermächtnismotiv operativ wird. Wie Weil (1987) zeigt, ist das in der Regel nicht der Fall. In diesem Zusammenhang sei hier auch auf Jaeger (1998) verwiesen. Jaeger untersucht den Einfluß intragenerational heterogener interpersonaler Diskontierungsraten auf die Gültigkeit des Äquivalenztheorems. Die endogen bestimmte interpersonale Diskontierungsrate kennzeichnet den Grad des Altruismus der Akteure, und dieser manifestiert sich in ihrem Vermächtnis- und Schenkungsverhalten. Auch Jaeger zeigt, daß das Äquivalenztheorem in der Regel nicht gilt. 


\section{Anhang B: Herleitung einer Tauschkurve}

Die Tauschkurve ist der geometrische Ort aller nutzenmaximierenden Konsumpläne, bei gegebenem Einkommen und variierender Verzinsung der Ersparnis. Die Form der Tauschkurve ist für die Dynamik der in den Kapiteln 3 bis 6 entwickelten Ökonomien von entscheidender Bedeutung. Aus diesem Grund soll die Herleitung einer Tauschkurve explizit dargestellt werden, um die ökonomischen Zusammenhänge, die ihre Gestalt determinieren, genau zu verstehen.

Die intertemporale Budgetrestriktion der Akteure lautet gemäß (3.4):

$$
c_{t}^{t}+\delta_{t+1} \cdot c_{t+1}^{t}=1-\varepsilon
$$

Aus der Nutzenmaximierung folgt gemäß (3.9):

$$
\frac{\partial u\left(c_{t}^{t}, c_{t+1}^{t}\right) / \partial c_{t+1}^{t}}{\partial u\left(c_{t}^{t}, c_{t+1}^{t}\right) / \partial c_{t}^{t}}=\delta_{t+1} \stackrel{\text { Budgetres. }}{\longrightarrow} \text { optimaler Konsumvektor }\left(c_{t}^{t}, c_{t+1}^{t}\right) \text {. }
$$

Darstellung B.1 zeigt das im Konsumraum dargestellte optimale Konsumverhalten.

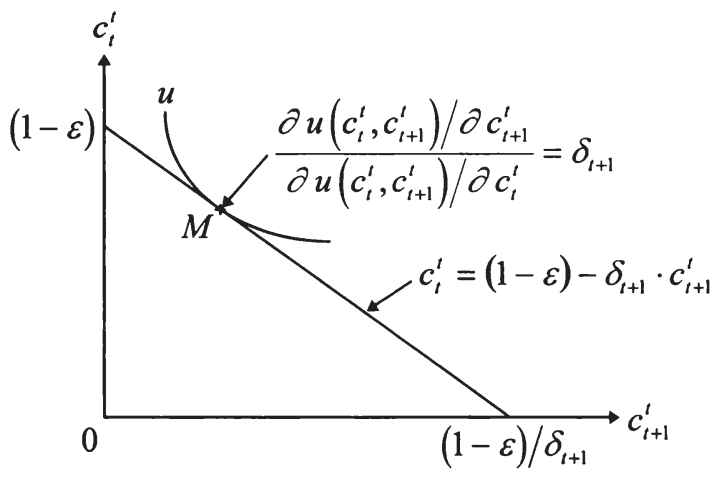

Darst. B.1: Konsumdiagramm 
Die Budgetgerade schneidet die Ordinate in $(1-\varepsilon)$ und die Abszisse in $(1-\varepsilon) / \delta_{t+1}$, und sie besitzt eine Steigung von $-\delta_{t+1}$. Der optimale Konsumplan eines Akteurs (in Darstellung B.1 mit $M$ bezeichnet) ist der Punkt auf seiner Budgetgeraden, den die Indifferenzkurve, die das von ihm maximal erreichbare Nutzenniveau kennzeichnet, tangiert. In diesem Punkt stimmen die ökonomische Substitutionsrate, also die Verzinsung der Ersparnis, und die Grenzrate der Substitution im Konsum überein. Das optimale Konsumverhalten läßt sich aber auch in Form von Überschußnachfragefunktionen formulieren. Eine Überschußnachfragefunktion gibt die Differenz zwischen der Konsumnachfrage und dem Einkommen an.

Die Überschußnachfragefunktion der jungen Akteure lautet somit:

$$
z_{t}^{t}\left(\delta_{t+1}\right):=c_{t}^{t}\left(\delta_{t+1}, 1-\varepsilon\right)-(1-\varepsilon)
$$

Die Überschußnachfragefunktion der alten Akteure lautet:

$$
z_{t+1}^{t}\left(\delta_{t+1}\right):=c_{t+1}^{t}\left(\delta_{t+1}, 1-\varepsilon\right)
$$

Die Überschußnachfrage eines jungen Akteurs ist die Differenz zwischen seiner Konsumnachfrage und seinem Arbeitseinkommen. Da die Akteure im Alter kein Einkommen beziehen, stimmt die Überschußnachfrage in diesem Lebensabschnitt mit der Konsumnachfrage überein. Die Überschußnachfragefunktionen besitzen folgende Eigenschaften:

1. Die Überschußnachfragefunktionen sind stetig. Die Stetigkeit folgt aus der strengen Konvexität der Indifferenzkurven, welche äquivalent zur angenommenen strengen Quasikonkavität der intertemporalen Nutzenfunktion ist. So variiert der Tangentialpunkt einer Indifferenzkurve und der Budgetmenge stetig, wenn man die Budgetgerade um den Einkommenspunkt dreht.

2. Die Überschußnachfragefunktionen sind homogen nullten Grades in den Preisen. Nur das intertemporale Preisverhältnis (der einperiodige Diskontfaktor) ist relevant, nicht aber die Absolutpreise in den einzelnen Perioden. 
3. Die Überschußnachfragefunktionen erfüllen das Gesetz von Walras, was bedeutet, daß der aggregierte Wert der Überschußnachfragen eines Akteurs identisch null ist. Dies folgt aus der Eigenschaft der Nichtsättigung, welche durch die strenge Monotonie der Präferenzordnung, diese ist äquivalent zur angenommenen Eigenschaft, daß die intertemporale Nutzenfunktion der Akteure streng ansteigend ist, impliziert wird. Die Akteure werden immer einen Konsumplan am Rand ihrer Budgetmenge, also auf der Budgetgeraden, wählen. Dies bedingt, daß sich die Budgetrestriktionen der Jugend und des Alters in eine intertemporale Budgetrestriktion transformieren lassen. Unter Verwendung der intertemporalen Budgetrestriktion (B.1) und den Definitionen der Überschußnachfragefunktionen (B.3) und (B.4) erhält man:

$$
z_{t}^{t}\left(\delta_{t+1}\right)+\delta_{t+1} \cdot z_{t+1}^{t}\left(\delta_{t+1}\right) \equiv 0 \quad(\text { Walras }- \text { Gesetz })
$$

In jeder Periode gibt es zwei Klassen von gehandelten Objekten. Dies ist zum einen das nutzenstiftende Gut und das sind zum anderen die Vermögenstitel. Insofern gibt es in jeder Periode zwei Märkte. Dies sind der Gütermarkt und der Kapitalmarkt. Aus dem Walras-Gesetz folgt nun, daß wenn einer der Märkte geräumt wird, es der andere auch ist. ${ }^{1}$ Diese Tatsache ermöglicht es, sich bei der Analyse der Gleichgewichtsdynamik auf einen Markt zu beschränken.

Für die Gestalt der Tauschkurve ist es nun von entscheidender Bedeutung, wie sich eine Veränderung des Diskontfaktors auf die Überschußnachfrage in den beiden Lebensabschnitten auswirkt. Gemäß der Slutsky-Dekomposition läßt sich die Auswirkung einer Veränderung des Diskontfaktors auf das Konsumverhalten in der Jugend und im Alter in zwei Effekte aufspalten. Dies ist zum einen der intertemporale Substitutionseffekt (ISE) und zum anderen der Einkommenseffekt (EE).

\footnotetext{
${ }^{1}$ Vergleicht man die Budgetrestriktion der Jugend und des Alters mit der intertemporalen Budgetrestriktion, so erkennt man, daß die Ersparnis in letzterer nicht mehr erscheint. Die Ersparnis determiniert das Verhalten auf dem Kapitalmarkt, da dort Kapital in Höhe der Ersparnis angeboten wird bzw. Vermögenstitel mit einem der Ersparnis entsprechendem Kurswert nachgefragt werden.
} 


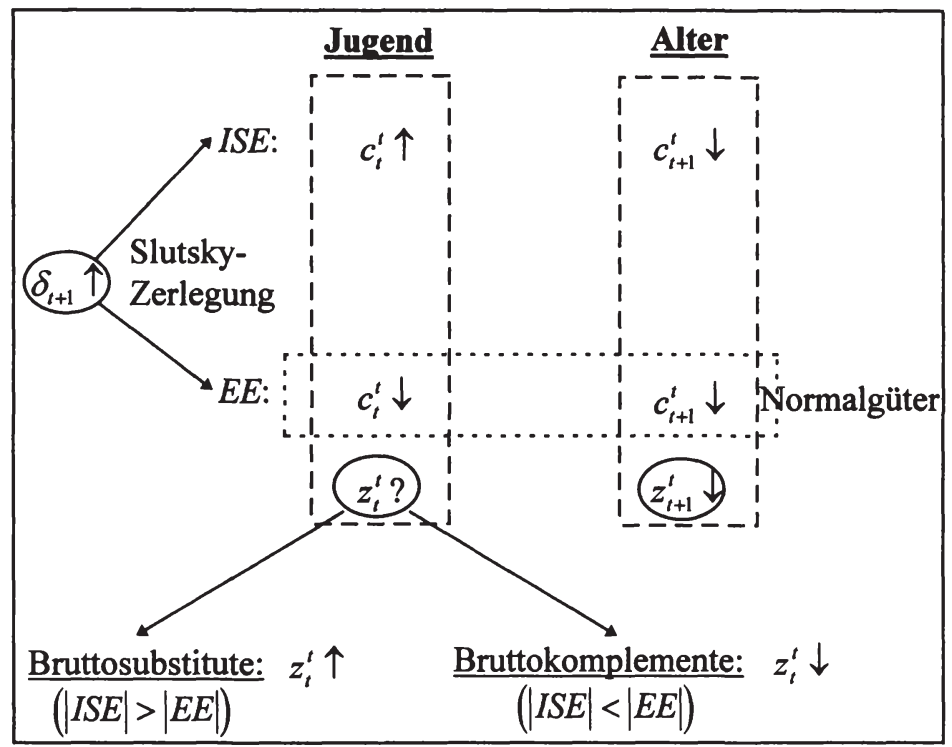

Darst. B.2: Auswirkung einer Diskontfaktorerhöhung

Darstellung B.2 veranschaulicht die Auswirkung einer Erhöhung des Diskontfaktors. Eine Erhöhung des Diskontfaktors wirkt über den ISE in Richtung einer Verlagerung des Konsums vom Alter in die Jugend (der Alterskonsum wird relativ teurer). Der EE wirkt in Richtung einer Reduktion des Konsums in beiden Lebensabschnitten (die Güter sind Normalgüter und die Budgetmenge verkleinert sich durch die Reduktion der Verzinsung der Ersparnis). ${ }^{1}$ Im Alter wirken beide Effekte somit in Richtung einer Reduktion des Konsums und damit in Richtung einer Reduktion der Überschußnachfrage. In der Jugend wirken beide Effekte entgegengesetzt. Überkompensiert der ISE den EE in der Jugend, so erhöht sich der Jugendkonsum und damit auch die Überschußnachfrage. Der Jugendkonsum und der Alterskonsum sind dann Bruttosubstitute. Überkompensiert der EE den ISE in der Jugend, so reduziert sich der Jugendkonsum und

${ }^{1}$ Der ISE läßt sich graphisch als Bewegung des Konsumplans entlang einer Indifferenzkurve veranschaulichen und der EE als eine Bewegung entlang des Einkommensexpansionspfades. Der Gesamteffekt ist die Vektoraddition aus beiden Effekten. 
damit auch die Überschußnachfrage. Der Jugendkonsum und Alterskonsum sind dann Bruttokomplemente.

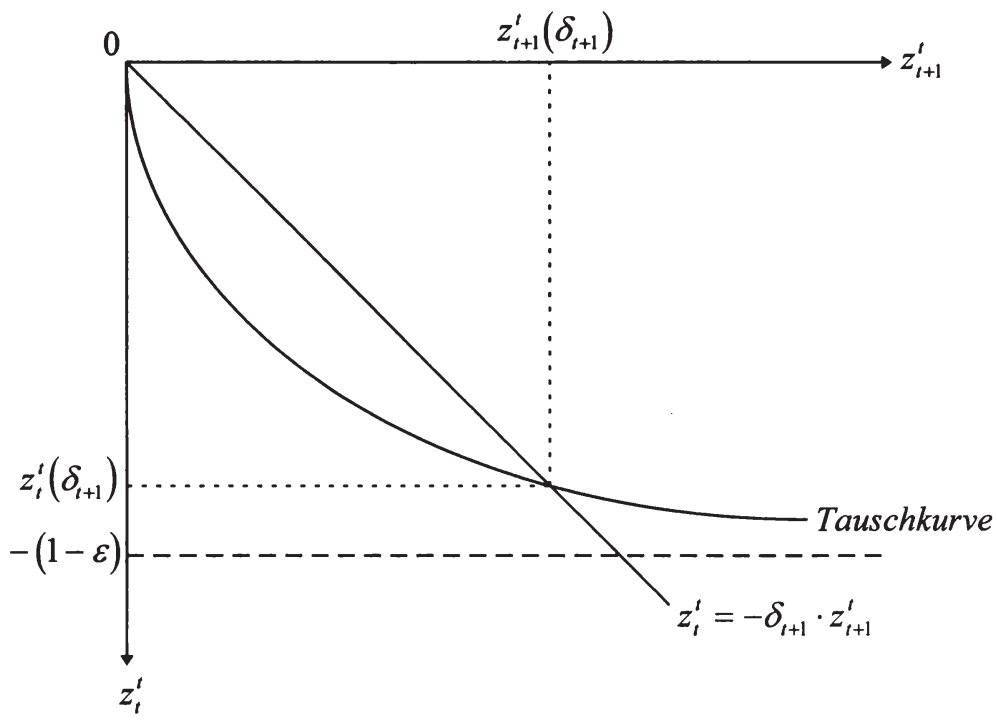

Darst. B.3: Überschußnachfragediagramm

Mit der Kenntnis dieser Zusammenhänge läßt sich die Tauschkurve im Überschußnachfrageraum herleiten. Sei hierfür Darstellung B.3 betrachtet. Der Ursprung des Überschußnachfragediagramms korrespondiert mit dem Einkommenspunkt im Konsumdiagramm. Auf der Ordinate ist die Überschußnachfrage in der Jugend abgetragen und auf Abszisse die im Alter. Die Überschußnachfrage in der Jugend kann minimal $-(1-\varepsilon)$ sein (dies ist das Negative des Arbeitseinkommen der jungen Akteure). Das Überschußangebot (= die Ersparnis) kann nicht höher als das verfügbare Einkommen sein. Die Überschußnachfrage im Alter kann nicht kleiner als null sein, da ein negativer Konsum nicht möglich ist. Somit ist der Verlauf der Tauschkurve auf den vierten Quadranten restringiert. Zur Budgetgeraden im Konsumdiagramm korrespondiert eine durch den 
Ursprung verlaufende Gerade, mit einer Steigung, die dem negativen des Diskontfaktors entspricht. Alle Punkte auf einer solchen Geraden, bis zur horizontalen unterbrochen gezeichneten Linie, erfüllen die Budgetrestriktion mit Gleichheitszeichen. Jedoch nur ein Punkt ist optimal, und dieser liegt auf der Tauschkurve (Definition der Tauschkurve). Erhöht sich nun der Diskontfaktor, reduziert sich die optimale Überschußnachfrage im Alter und erhöht bzw. reduziert sich in der Jugend, je nachdem, ob die Güter Bruttosubstitute sind oder Bruttokomplemente. Darstellung B.3 zeigt den Fall, in dem die Güter im gesamten Bereich Bruttosubstitute sind. Dies ist genau dann der Fall, wenn die Steigung der Tauschkurve über ihren gesamten Verlauf negativ ist. Sind die Güter in einem bestimmten Bereich Bruttokomplemente, so ist die Steigung der Tauschkurve in diesem Bereich positiv.

Aus dem Walras-Gesetz (B.5) folgt:

$$
\frac{z_{t}^{t}\left(\delta_{t+1}\right)}{z_{t+1}^{t}\left(\delta_{t+1}\right)}=-\delta_{t+1} .
$$

Gemäß Gleichung (B.6) bewirkt eine Reduktion des Diskontfaktors eine betragsmäßige Reduktion des Verhältnisses der Überschußnachfrage in der Jugend zur Überschußnachfrage im Alter. Die Tauschkurve weist somit einen elastischen Verlauf auf. Ökonomisch steckt dahinter die Tatsache, daß der ISE und der EE in der Jugend entgegengesetzt wirken und im Alter gleichgerichtet. Eine durch den Ursprung gehende Gerade kann die Tauschkurve somit höchstens zweimal schneiden. Einmal im Ursprung und ein weiteres mal im vierten Quadranten. Es ist der elastische Verlauf der Tauschkurven, der die Existenz und Eindeutigkeit der langfristigen Gleichgewichte der in den Kapiteln 3 bis 6 entwickelten Modell-Ökonomien gewährleistet.

Um die Gewinnung einer Tauschkurve noch besser zu verstehen, soll im Folgenden ein Beispiel berechnet werden. Sei hierfür angenommen, daß die Präfe- 
renzordnung der Akteure durch folgende additiv separable intertemporale Nutzenfunktion repräsentiert werden kann: ${ }^{1}$

$$
u\left(c_{t}^{t}, c_{t+1}^{t}\right)=c_{t}^{t}+\left(c_{t+1}^{t}\right)^{\frac{1}{2}}
$$

Der Exponent des Alterskonsums spiegelt die positive Zeitpräferenz der Akteure wieder. Die Lagrangefunktion lautet:

$$
L=c_{t}^{t}+\left(c_{t+1}^{t}\right)^{\frac{1}{2}}+\lambda \cdot\left(1-\varepsilon-c_{t}^{t}-\delta_{t+1} \cdot c_{t+1}^{t}\right)
$$

Die Bedingungen erster Ordnung lauten:

$$
\begin{aligned}
& \frac{\partial L}{\partial c_{t}^{t}}=1-\lambda \stackrel{!}{=} 0, \\
& \frac{\partial L}{\partial c_{t+1}^{t}}=\frac{1}{2} \cdot\left(c_{t+1}^{t}\right)^{-\frac{1}{2}}-\lambda \cdot \delta_{t+1} \stackrel{!}{=} 0, \\
& \frac{\partial L}{\partial \lambda}=1-\varepsilon-c_{t}^{t}-\delta_{t+1} \cdot c_{t+1}^{t} \stackrel{!}{=} 0 .
\end{aligned}
$$

Aus den Bedingungen erster Ordnung erhält man die Konsumnachfragefunktionen:

$$
\begin{aligned}
& c_{t}^{t}\left(\delta_{t+1}, 1-\varepsilon\right)=(1-\varepsilon)-\frac{1}{4 \cdot \delta_{t+1}}, \\
& c_{t+1}^{t}\left(\delta_{t+1}, 1-\varepsilon\right)=\frac{1}{4 \cdot\left(\delta_{t+1}\right)^{2}} .
\end{aligned}
$$

${ }^{1}$ Der interessierte Leser kann sich davon überzeugen, daß die Nutzenfunktion streng quasikonkav und streng ansteigend ist. 
Die Überschußnachfragefunktionen lauten damit:

$$
\begin{aligned}
& z_{t}^{t}\left(\delta_{t+1}\right)=-\frac{1}{4 \cdot \delta_{t+1}}, \\
& z_{t+1}^{t}\left(\delta_{t+1}\right)=\frac{1}{4 \cdot\left(\delta_{t+1}\right)^{2}} .
\end{aligned}
$$

Unter Zuhilfenahme des Walras-Gesetzes erhält man dann schließlich die Gleichung für die Tauschkurve:

$$
z_{t}^{t}=-\frac{1}{2} \cdot\left(z_{t+1}^{t}\right)^{\frac{1}{2}}
$$

Die Tauschkurve hat ihren Ursprung im Einkommenspunkt und weist im gesamten Bereich eine negative Steigung auf. Die Güter sind somit Bruttosubstitute. Von der Form her entspricht die Tauschkurve der in Darstellung B.3 gezeichneten.

Im Haupttext wird, zur Diskussion der Modelldynamik, die in das Konsumdiagramm übertragene Tauschkurve verwendet. Die Transformation der Tauschkurve vom Überschußnachfrageraum in den Konsumraum ist jedoch trivial, da der Ursprung des Überschußnachfragediagramms mit dem Einkommenspunkt im Konsumdiagramm übereinstimmt. 


\section{Anhang C: Die Theorie der offenbarten Präferenz}

Das übliche Vorgehen zur Beschreibung des Nachfrageverhaltens von Akteuren ist anzunehmen, daß diese eine Nutzenfunktion besitzen, die sie versuchen, unter Einhaltung ihrer Budgetrestriktion, zu maximieren. Ein alternativer Ansatz ist der von Samuelson (1947). ${ }^{1}$ Dieser Ansatz, den Samuelson Theorie der offenbarten Präferenz nennt, definiert ein Rationalitätsprinzip, das auf beobachtetem Verhalten basiert. Ein Akteur, der diesem Rationalitätsprinzip gehorcht, verhält sich so als ob er eine Nutzenfunktion, unter Einhaltung einer Budgetrestriktion, maximieren würde. Im Haupttext wird die Theorie der offenbarten Präferenz verwandt, um Wohlfahrtsvergleiche anzustellen. Aus diesem Grund soll die Theorie im Folgenden skizziert werden.

Seien der Preisvektor und der Konsumplan wie folgt definiert:

$$
\delta:=\left(1, \delta_{t+1}\right) \text { und } c:=\left(\begin{array}{c}
c_{t}^{t} \\
c_{t+1}^{t}
\end{array}\right)
$$

Gegeben seien der Preisvektor $\delta$ und die beiden sich unterscheidenden Konsumpläne $c$ und $\bar{c}$. Gilt $\delta \cdot c \geq \delta \cdot \bar{c}$ und wählt der Akteur $c$ und nicht $\bar{c}$, dann sagt man, daß der Akteur $c$ gegenüber $\bar{c}$ offenbart präferiert. Das Samuelson'sche Rationalitätsprinzip besagt nun folgendes:

Wenn $c$ gegenüber $\bar{c}$ offenbart präferiert wird und $c \neq \bar{c}$ gilt, dann kann es nicht der Fall sein, daß $\bar{c}$ gegenüber $c$ offenbart präferiert wird.

Dies ist das schwache Axiom der offenbarten Präferenz. Es besagt, daß wenn $c$ und $\bar{c}$ durchführbar sind und $c$ gewählt wird, keine Budgetmenge existiert, die $c$ und $\bar{c}$ enthält, bei der $\bar{c}$ anstatt $c$ gewählt wird. Dieses ökonomische Rationalitätsprinzip läßt sich im Konsumdiagramm graphisch veranschaulichen.

\footnotetext{
${ }^{1}$ Vgl. Samuelson (1947), Kapitel 5.
} 


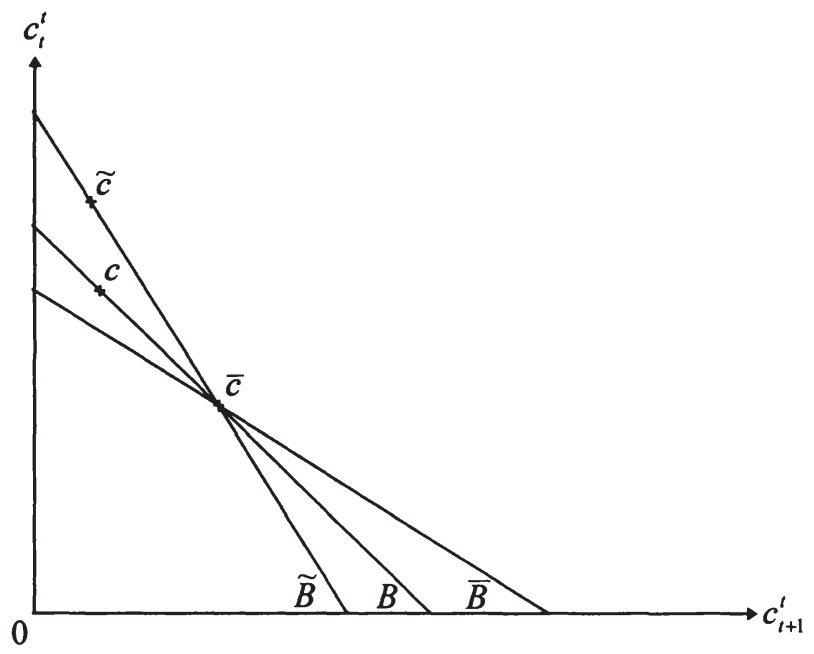

Darst. C.1: Offenbarte Präferenz

Darstellung C.1 zeigt drei Konsumpläne und drei Budgetmengen, die durch die sie begrenzenden Budgetgeraden veranschaulicht werden. Sei angenommen, die Budgetrestriktion des Akteurs ist $B$ und er wählt $c$. Er hätte auch $\bar{c}$ wählen können, somit wird $c$ gegenüber $\bar{c}$ offenbart präferiert. Wählt er bei einer anderen Budgetrestriktion $\bar{c}$, so darf $c$ mit dieser Budgetrestriktion nicht wählbar sein. Ansonsten würde das Axiom der offenbarten Präferenz verletzt werden. Eine solche Budgetrestriktion ist zum Beispiel $\bar{B}$. Ist die Budgetrestriktion des Akteurs zum Beispiel $\widetilde{B}$, so kann es nicht sein, daß er $\bar{c}$ wählt. Dies wäre eine Verletzung des Axioms der offenbarten Präferenz, da bei $\widetilde{B} c$ und $\bar{c}$ wählbar sind. Ist die Budgetrestriktion des Akteurs $\widetilde{B}$ und er wählt $\widetilde{c}$ und nicht $c$, so stellt dies keine Verletzung des Rationalitätsprinzips dar. Denn $\widetilde{c}$ ist mit $B$ nicht wählbar. Man erkennt, daß im Rahmen der Theorie der offenbarten Präferenz, die Ordnung von Güterbündeln aufgrund der beobachtbaren Reaktion, auf die Veränderung der Budgetrestriktion, erfolgt, ohne Rückgriff auf die Existenz einer Nutzenfunktion. 
Wird nun $c$ gegenüber $\bar{c}$ offenbart präferiert, so impliziert dies, daß der durch $c$ erzeugte Nutzen mindestens so hoch ist wie der durch $\bar{c}$ erzeugte, also $u(c)$ $\geq u(\bar{c})$ gilt. Der Grund ist folgender: $c$ und $\bar{c}$ waren durchführbar $(\delta \cdot c \geq \delta \cdot \bar{c})$, der Akteur hat aber $c$ gewählt. Aus der Nutzenmaximierungshypothese folgt dann $u(c) \geq u(\bar{c})$.

Gilt $\delta \cdot c>\delta \cdot \bar{c}$ und der Akteur wählt $c$, so sagt man, daß der Akteur $c$ gegenüber $\bar{c}$ streng offenbart präferiert. Dies impliziert, daß der durch $c$ erzeugte Nutzen echt höher ist als der durch $\bar{c}$ erzeugte. Es gilt also $u(c)>u(\bar{c})$. Diese Implikation folgt aus der Nutzenmaximierungshypothese in Verbindung mit der lokalen Nichtsättigung. ${ }^{1}$ Aufgrund des vorherigen Absatzes ist klar, daß $u(c)$ $\geq u(\bar{c})$ gilt. Sei nun angenommen, daß $u(c)=u(\bar{c})$ gilt. Aufgrund der lokalen Nichtsättigung existiert dann ein nahe bei $c$ liegender Konsumplan $\hat{c}$ mit folgender Eigenschaft: $\delta \cdot c>\delta \cdot \hat{c}$ und $u(\hat{c})>u(c)=u(\bar{c})$. Dies stellt einen Widerspruch zur Nutzenmaximierungshypothese dar. Somit impliziert die Tatsache, daß $c$ gegenüber $\bar{c}$ streng offenbart präferiert, daß $c$ echt nutzenstiftender ist als $\bar{c}$.

\footnotetext{
${ }^{1}$ Die lokale Nichtsättigung wird durch die strenge Monotonie der Präferenzordnung impliziert. Letztere ist äquivalent zur Annahme, daß die intertemporale Nutzenfunktion der Akteure streng ansteigend ist.
} 
Michael Ganske - 978-3-631-75472-6

Downloaded from PubFactory at 01/11/2019 04:35:58AM

via free access 


\section{Literaturverzeichnis}

Alogoskoufis, G. S. und F. van der Ploeg (1991), On budgetary policies, growth, and external deficits in an interdependent world, Journal of the Japanese and International Economies, 5, S. 305-324

Arifovic, J. (1996), The behavior of the exchange rate in the genetic algorithm and experimental economies, Journal of Political Economy, 104, S. 510-541

Arrow, K. J. und G. Debreu (1954), Existence of an equilibrium for a competitive economy, Econometrica, 22, S. 265-290

Asea, P. K. und S. J. Turnovsky (1998), Capital income taxation and risk-taking in a small open economy, Journal of Public Economics, 68, S. 55-90

Azariadis, C. (1993), Intertemporal Macroeconomics, Oxford / Cambridge: Blackwell Publishers

Azariadis, C. und R. Guesnerie (1986), Sunspots and cycles, Review of Economic Studies, 53, S. 725-737

Balasko, Y. und C. Ghiglino (1995), On the existence of endogenous cycles Journal of Economic Theory, 67, S. 566-577

Balasko, Y. und K. Shell (1980), The overlapping-generations model,I:

The case of pure exchange without money, Journal of Economic Theory, 23, S. 281-306

Balasko, Y. und K. Shell (1981), The overlapping-generations model. II. The case of pure exchange with money, Journal of Economic Theory, 24, S. 112-142

Balasko, Y. et al. (1980), Existence of competitive equilibrium in a general overlapping-generations model, Journal of Economic Theory, 23, S. 307-322

Barro, R. J. (1974), Are government bonds net wealth?, Journal of Political Economy, 82, S. 1095-1117 
Barro, R. J. und X. Sala-i-Martin (1995), Economic Growth, New York u.a.: McGraw-Hill

Barten, A. P. und V. Böhm (1982), Consumer theory, in: K.J. Arrow und M.D. Intriligator (eds.), Handbook of Mathematical Economics, vol. II, Amsterdam: North-Holland, S. 381-429

Batina, R. G. und T. Ihori (1991), International spillover effects of consumption taxation, Journal of the Japanese and International Economies, 5, S. 404-420

Baumol, W .J. und J. Benhabib (1989), Chaos: Significance, mechanism, and economic application, Journal of Economic Perspectives, 3, S. 77-105

Benhabib, J. und R. H. Day (1982), A characterization of erratic dynamics in the overlapping generations Model, Journal of Economic Dynamics and Control, 4, S.37-55

Bental, B. (1985), Is capital mobility always desirable? A welfare analysis of portfolio autarkie in a growing economy, International Economic Review, 26, S. 203-212

Bertocchi, G. und Y. Wang (1995), The real value of money under endogenous beliefs, Journal of Economic Theory, 67, S. 205-222

Bianconi, M. (1995), Fiscal policy in a simple two-country dynamic model, Journal of Economic Dynamics and Control, 19, S. 395-419

Bianconi, M. und S. J. Turnovsky (1997), International effects of government expenditure in interdependent economies, Canadian Journal of Economics, 30, S. 57-84

Blanchard, O. J. (1985), Debt, deficits, and finite horizons, Journal of Political Economy, 93, 223-247

Blanchard, O. J. und S. Fisher (1989), Lectures on Macroeconomics, Cambridge / London: The MIT Press

Boldrin, M. und M. Woodford (1990), Equilibrium models displaying endogenous fluctuations and chaos, Journal of Monetary Economics, 25 , S. $189-222$ 
Bovenberg, A. L. (1992), Residence- and source-based taxation of capital income in an overlapping generations model, Journal of Economics, 56, S. 267-295

Bovenberg, A. L. (1994), Capital taxation in the world economy, in: F. van der Ploeg (ed.): The Handbook of International Macroeconomics, Oxford / Cambridge: Blackwell, S. 116-150

Bovenberg, A. L. und C. van Ewijk (1997), Progressive taxes, equity, and human capital accumulation in an endogenous growth model with overlapping generations, Journal of Public Economics, 64, S. 153-179

Brealey, A. und S. C. Myers (1991), Principles of Corporate Finance, Fourth Edition, New York u.a.: McGraw-Hill

Breyer, F. (1998), On the Pareto-improving Abolition of unfunded public pension systems: An application of growth theory, in: K.-J. Koch und K. Jaeger (eds.), Trade, Growth, and Economic Policy in Open Economies, Berlin u.a.: Springer, S. 123-137

Brock, W. A. (1990), Overlapping generations models with money and transactions costs, in: B. M. Friedman und F. H. Hahn (eds.), Handbook of Monetary Economics, vol. I, Amsterdam u.a.: North-Holland, S. 263-295

Buiter, W. H. (1981), Time preference and international lending and borrowing in an overlapping-generations model, Journal of Political Economy, 89, S. 769-797

Buiter, W. H. (1987), Fiscal policy in open, interdependent economies, in: A. Razin und E. Sadka (eds.), Economic Policy in Theory and Practice, Houndmills u.a.: Macmillan Press, S. 101-144

Buiter, W. H. und K. M. Kletzer (1991), The welfare economics of cooperative and noncooperative fiscal policy, Journal of Economic Dynamics and Control, 15, S. 215-244

Burbidge, J. B. und W. M. Scarth (1995), Eliminating interest taxation and tariffs: The underpinnings for recent canadian policy, Canadian Journal of Economics, 28, S. 437-449 
Burke, J. L. (1987), Inactive transfer policies and efficiency in general overlapping-generations economies, Journal of Mathematical Economics, 16, S. 201-222.

Caballe, J. (1998), Growth Effects of Taxation under Altruism and Low Elasticity of Intertemporal Substitution, The Economic Journal, 108, S. 92-104

Calvo, G. A. (1978), On the indeterminacy of interest rates and wages with perfect forsight, Journal of Economic Theory, 19, S. 321-337

Calvo, G. A. und M. Obstfeld (1988), Optimal time-consistent fiscal policy with finite lifetimes: Analysis and extensions, in: E. Helpman u.a. (eds.): Economic Effects of the Government Budget, Cambridge / London: Harvard University Press, S. 163-198

Cass, D. (1965), Optimum growth in an aggregative model of capital accumulation, Review of Economic Studies, 32, S. 233-240

Cass, D. (1972), On capital overaccumulation in the aggregative, neoclassical model of economic growth: A complete characterization, Journal of Economic Theory, 4, S. 200-223

Cass, D. und K. Shell (1980), In defense of a basic approach, in: J. H. Kareken und N. Wallace (eds.): Models of Monetary Economies, Minneapolis, Min.: Federal Reserve Bank of Minneapolis, S. 251-260

Cass, D. und M. E. Yaari (1966), A re-examination of the pure consumption loans model, Journal of Political Economy, 74, S. 353-367

Cass, D. et al. (1979), The role of money in supporting the pareto optimality of competitive equilibrium in consumption-loan type model, Journal of Economic Theory, 20, S. 41-80

Chiang, A. C. (1984), Fundamental Methods of Mathematical Economics, $3^{\text {rd }}$ ed., Auckland u.a.: McGraw-Hill

Chiang, A. C. (1992), Elements of Dynamic Optimization, Auckland u.a.: McGraw-Hill 
Christensen, T. A. und S. B. Nielsen (1995), International repercussions of source-based capital income taxation, Journal of Economic Integration, 10, S. $87-110$

Clower, R. (1967), A reconsideration of the microfoundations of monetary theory, Western Economic Journal, 6, S. 1-8

Crettez, B. et al. (1998), Time preference and capital mobility in an OLG model with land, Journal of Population Economics, 11, S. 149-158

Dahan, M. und Z. Hercowitz (1998), Fiscal policy and saving under distortionary taxation, Journal of Monetary Economics, 42, S. 25-45

Daniel, B. C. (1997), Precautionary saving and persistent current account imbalance, Journal of International Economics, 42, S. 179-193

Day, R. H. (1994), Complex Economic Dynamics, Volume I: An Introduction to Dynamical Systems and Market Mechanisms, Cambridge / London: The MIT Press

Devereux, M. B. und S. Shi (1991), Capital accumulation and the current account in a two-country model, Journal of International Economics, 30, S. $1-25$

Diamond, P. E. (1965), National debt in a neoclassical growth model, American Economic Review, 55, S. 1126-1150

Dixit, A. K. (1990), Optimization in Economic Theory, Oxford u.a.: Oxford University Press

Dornbusch, R. (1985), Intergenerational and international trade, Journal of International Economics, 18, S. 123-139

Eaton, J. (1988), Foreign-owned land, The American Economic Review, 78, S. 76-88

Epstein, L. G. und J. A. Hynes (1983), The rate of time preference and dynamic economic analysis, Journal of Political Economy, 91, S. 611-635 
Ethier, W. J. (1994), Moderne Außenwirtschaftstheorie, 3. Auflage, München / Wien: Oldenburg Verlag

Farmer, R. E. A. (1993), The Macroeconomics of Self-Fulfilling Prophecies, Cambridge / London: The MIT Press

Feenstra, R. C. (1986), Functional equivalence between liquidity costs and the utility of money, Journal of Monetary Economics, 17, S. 271-291

Fisher, E. O’N (1995), Growth, trade, and international transfers, Journal of International Economics, 39, S. 143-158

Freeman, S. und R. G. Murphy (1989), Inside money and the open economy, Journal of International Economics, 26, S. 29-51

Frenkel, J. A. u.a. (1991), International Taxation in an Integrated World, Cambridge / London: The MIT Press

Frenkel, J. A. und A. Razin (1985a), Government spending, debt, and international economic interdependence, The Economic Journal, 95, S. 619-636

Frenkel, J. A. und A. Razin (1985b), Fiscal expenditures and international economic interdependence, in: W. H. Buiter und R. C. Marston (eds.), International Economic Policy Coordination, Cambridge u.a.: Cambridge University Press, S. 37-73

Frenkel, J. A. und A. Razin (1986a), The international transmission and effects of fiscal policies, American Economic Review (Papers and Proceedings), 76, S. 330-335

Frenkel, J. A. und A. Razin (1986b), Fiscal policies in the world economy, Journal of Political Economy, 94, S. 564-594

Frenkel, J. A. und A. Razin (1987), The international transmission of fiscal expenditures and budget deficits in the world economy, in: A. Razin und E. Sadka (eds.): Economic Policy in Theory und Practice, Houndmills u.a.: Macmillan Press, S. 51-96 
Frenkel, J. A. und A. Razin (1996), Fiscal Policies and Growth in the World Economy, $3^{\text {th }}$ ed., Cambridge / London: The MIT Press

Fried, J. (1980), The international distribution of the gains from technical change and from international trade, Canadian Journal of Economics, 13, S. $65-81$

Fried, J. und P. Howitt (1988), Fiscal deficits, international trade and welfare, Journal of International Economics, 24, S. 1-22

Gagay, F. et al. (1986), Monetary and fiscal policies in a general equilibrium model, Journal of Economic Theory, 39, S. 329-357

Gale, D. (1971), General equilibrium with imbalance of trade, Journal of International Economics, 1, S. 141-158

Gale, D. (1973), Pure exchange equilibrium of dynamic economic models, Journal of Economic Theory, 6, S. 12-36

Gale, D. (1974), The trade imbalance story, Journal of International Economics, 4, S. 119-137

Geanakoplos, J. D. (1987a), Overlapping generations model of general equilibrium, in: J. Eatwell u.a. (eds.), The New Palgrave - A Dictionary of Economics, London u.a.: MacMillan Press, S. 767-779

Geanakoplos, J. D. (1987b), Arrow-Debreu model of general equilibrium, in: J. Eatwell u.a. (eds.), The New Palgrave - A Dictionary of Economics, London u.a.: MacMillan Press, S. 116-123

Geanakoplos, J. D. und H. M. Polemarchakis (1986), Walrasian indeterminacy and Keynesian macroeconomics, Review of Economic Studies, 1986, S. 755-779

Geanakoplos, J. D. und H. M. Polemarchakis (1991), Overlapping generations, in: W. Hildenbrand und H. Sonnenschein (eds.), Handbook of Mathematical Economics, vol. IV, Amsterdam u.a.: North-Holland, S. 1899-1960

Grandmont, J.-M. (1985), On endogenous competitive business cycles, Econometrica, 53, S. 995-1045 
Grandmont, J.-M. (1993), Expectations driven nonlinear business cycles, Rheinisch-Westfälische Akademie der Wissenschaft Vorträge, 397, S. 7-32

Grandmont, J.-M. und G. Laroque (1986), Stability of cycles and expectations, Journal of Economic Theory, 40, S. 138-151

Grandmont, J.-M. und Y. Younes (1973), On the role of money and the existence of monetary equilibrium, Review of Economic Studies, 39, S. $355-372$

Hartley, J. E. (1997), The Representative Agent in Macroeconomics, London: Routledge

Helpman, E. und A. Razin (1982), Dynamics of a floating exchange rate regime, Journal of Political Economy, 90, S. 728-754

Helpman, E. und A. Razin (1987), Exchange rate management: Intertemporal tradeoffs, The American Economic Review, 77, S. 103-107

Helpman, E. und E. Sadka (1979), Optimal financing of the government's budget: Taxes, bonds, or money?, The American Economic Reviei, 69 , S. $152-160$

Hogg, R. V. und A. T. Craig (1978), Introduction to Mathematical Statistics, $4^{\text {th }}$ ed., Cambridge / London: The MIT Press

Ihori, T. (1991), Capital income taxation in a world economy: A territorial system versus a residence system, The Economic Journal, 101, S. 958-965

Issing, O. (1992), Einführung in die Geldpolitik, 4., überarbeitete Auflage, München: Vahlen

Itaya, J.-I. (1995), Dynamic tax incidence in a finite horizon model, Public Finance, 50, S. 246-266

Iwamoto, Y. und A. Shibata (1991), Capital income taxation in the current account in a small open economy, Journal of International Money and Finance, 10, S. 480-496 
Jaeger, K. (1998), An overlapping-generations model with heterogenous bequest and gift motives, in: K.-J. Koch und K. Jaeger (eds.), Trade, Growth, and Economic Policy in Open Economies, Berlin u.a.: Springer, S. 139-163

Kareken, J. und N. Wallace (1977), Portfolio autarkie: A welfare analysis, Journal of International Economics, 7, S. 19-43

Kareken, J. und N. Wallace (1981), On the indeterminacy of equilibrium exchange rates, Quarterly Journal of Economics, 96, S. 207-222

Keen, M. (1990), Welfare effects of capital income tax reform in a growing world economy, Public Finance, 45, S. 283-303

Kehoe, T. J. (1989), Intertemporal general equilibrium models, in: F. Hahn (ed.), The Economics of Missing Markets, Information, and Games, Oxford: Clarendon Press, S. 363-393

Kehoe, T. J. et al. (1991), Gross substitutability in large-square economies, Journal of Economic Theory, 54, S. 1-25

Kemp, M. C. und Wong K.-y (1995), Gains from trade with overlapping generations, Economic Theory, 6, S. 283-303

Kim. K.-S. und J. Lee (1997), Reexamination of dynamic efficiency with taxation on land, Economics Letters, 57, S. 169-175

Kirman, A. P. (1992), „Whom or what does the representative individual represent?", Journal of Economic Perspectives, 6, S. 117-136

Koopmans, T. C. (1965), On the concept of optimal economic growth in: The Econometric Approach to Development Planning, Amsterdam: North-Holland, S. 225-287

Lin, S. (1994a), Budget deficits, time preference, and the external deficits, The Economic Studies Quarterly, S. 289-305

Lin, S. (1994b), Capital taxation and accumulation in a growing world Economy with Deficit Finance, International Tax and Public Finance, 1, S. 127-145 
Lin, S. (1998), Labor income taxation and human capital accumulation, Journal of Public Economics, 68, S. 291-302

Linde, R. (1981), Produktion II: Produktionsfunktionen, in: W. Albers u.a. (Hrsg.): Handwörterbuch der Wirtschaftswissenschaft (HdWW), Bd. 6, Stuttgart u.a.: Fischer u.a., S. 276-295

Lucas, R. E., Jr. (1976), Econometric policy evaluation: A critique, in: K. Brunner und A. H. Meltzer (eds.), The Phillips Curve and Labor Markets, Carnegie Rochester Conference Series on Public Policy, Amsterdam: North-Holland, S. 19-46

Lucas, R. E., Jr. (1980), Equilibrium in a pure currency economy, in: J. Kareken und N. Wallace (eds.), Models of Monetary Economies, Minneapolis: Federal Reserve Bank of Minneapolis, S. 131-145

Lucas, R. E., Jr. (1990), Supply-side economics: An analytical review, Oxford Economic Papers, 42, S. 293-316

Malinvaud, E. (1995), Maurice Allais, unrecognized pioneer of overlapping generations models, in: B.R. Munier (ed.): Markets, Risk and Money Essays in Honor of Maurice Allais, Dordrecht u.a.:

Kluwer Academic Publishers, S. 111-128

Mansoorian, A. (1998), Habits, and durability in consumption, and the dynamics of the current account, Journal of International Economics, $44,69-82$

Manuelli, R. E. und J. Peck (1990), Exchange rate volatility in an equilibrium asset pricing model, International Economic Review, 31, 559-574

Mas-Colell, A. et al. (1995), Microeconomic Theory, New York / Oxford: Oxford University Press

Matsuyama, K. (1988), Terms-of-trade, factor intensities and the current account in a life-cycle model, Review of Economic Studies, 55, S. 247-262

Minford, P. (1992), Rational Expectations Macroeconomics: An Introductory Handbook, $2^{\text {nd }}$ ed., Oxford / Cambridge: Blackwell 
Neumann, M. (1990), Zukunftsperspektiven im Wandel - Lange Wellen in Wirtschaft und Politik, Tübingen: Mohr / Siebeck

Nicholson, W. (1992), Microeconomic Theory - Basic Principles and Extensions, $5^{\text {th }}$ ed., Orlando u.a.: The Dryden Press

Nielsen, S. B. (1992), Capital income taxation in a growing world economy, Journal of Economics, 55, S. 77-99

Nielsen, S. B. und P. B. Sorensen (1991), Capital income taxation in a growing open economy, European Economic Review, 34, S. 179-197

Nielsen, S. B. und P. B. Sorensen (1994), Inflation, capital taxation, and housing: The long run in a small open economy, Canadian Journal of Economics, 27, S. 198-217

Obstfeld, M. (1981), Macroeconomic policy, exchange-rate dynamics, and optimal asset accumulation, Journal of Political Economy, 89, S. 1142-1161

Obstfeld, M. und K. Rogoff (1996), Foundations of International Macroeconomics, Cambridge / London.: The MIT Press

Persson, T. (1985), Deficits and international welfare in open economies, Journal of International Economics, 19, S. 67-84

Persson, T. und L. E. O. Svensson (1985), Current account dynamics and the terms of trade: Harberger-Laursen-Metzler two generations later Journal of Political Economy, 93, S. 43-65

Piekkola, H. (1995), Capital income taxation, tax criteria, and intergenerational welfare, Journal of Economics, 62, S. 295-322

Ramsey, F. P. (1928), A mathematical theory of saving, Economic Journal, 38, S. 543-559

Rawls, J. (1971), A Theory of Justice, Cambridge / London: Harvard University Press

Renström, T. I. (1996), Endogenous taxation: An overlapping generations approach, The Economic Journal, 106, S. 471-482 
Romer, D. (1996), Advanced Macroeconomics, New York u.a.: McGraw-Hill

Ruffin, R. J. und Y. D. Yoon (1993), International capital movements in the Solow and overlapping generations growth models, Review of International Economics, 1, S. 123-135

Sachs, J. (1982), The current account in the macroeconomic adjustment process, Scandinavian Journal of Economics, 84, S. 147-159

Samuelson, P. A. (1947), Foundations of Economic Analysis, Cambridge / London: Harvard University Press

Samuelson, P. A. (1958), An exact consumption-loan model of interest with or without the social contrivance of money, The Journal of Political Economy, 66, S. 467-482

Sargent, T. J. (1987), Dynamic Macroeconomic Theory, Cambridge / London: Harvard University Press

Serra, P. (1991), Short-run and long-run welfare implications of free trade, Canadian Journal of Economics, 24, S. 21-33

Schittko, U. K. und B. Eckwert (1988), Intertemporal aspects in an aggregated two-country monetary macro model, Empirica, 15, S. 77-94

Schmid, M. (1988), Fiscal strategies, foreign indebtedness, and overlapping generations, Empirica, 15, S. 95-115

Schmid, M. und H. Großmann (1986), Auslandsverschuldung im Modell mit überlappenden Generationen, in: R. Ertel und H.-J. Heinemann (Hrsg.), Aspekte internationaler Wirtschaftsbeziehungen-Theoretische Erörterungen und empirische Befunde, NIW- Vortragsreihe, 2, Hannover: Niedersächsisches Institut für Wirtschaftsforschung, S. 23-59

Shell, K. (1971), Notes on the economics of infinity, Journal of Political Economy, 79, S. 1002-1011

Sibert, A. (1990), Taxing capital in a large, open economy, Journal of Public Economics, 41, S. 297-317 
Siebert, H. (1991), Außenwirtschaft, 5.Auflage, Stuttgart: Gustav Fischer Verlag

Starrett, D. A. (1972), On golden rules, the „biological theory of interest,“ and competitive inefficiency, Journal of Political Economy, 80, S. 276-291

Steigum, E., Jr. (1993), Accounting for long-run effects of policy by means of computable overlapping generations models, in: S. Honkapohja und Ingeberg (eds.), Macroeconomic Modelling and Policy Implications, Contributions to Economic Analysis, 216, Amsterdam: North-Holland, S. 45-67

Steiner, M. und C. Bruns (1996), Wertpapiermanagement, 5. Auflage, Stuttgart: Schäffer-Poeschel

Summers, L. H. (1981), Capital taxation and accumulation in a life cycle growth model, American Economic Review, 71, S. 533-544

Takayama, A. (1994), Analytical Methods in Economics, New York u.a.: Harvester Wheatsheaf

Tan, Kim-Heng (1997), Can budget deficits improve welfare in both the short run and long ran?, The Economic Record, 73, S. 16-21

Tirole, J. (1985), Asset bubbles and overlapping generations, Econometrica, 53, S. $1499-1528$

Tobin, J. (1980), Discussion, in: J. Kareken und N. Wallace (eds.), Models of Monetary Economies, Minneapolis: Federal Bank of Minneapolis, S. 83-90

Turnovsky, S. J. (1995), Methods of Macroeconomic Dynamics, Cambridge / London: The MIT Press

Turnovsky, S. J. (1997), International Macroeconomic Dynamics, Cambridge / London: The MIT Press

Varian, H. R. (1992), Microeconomic Analysis, $3^{\text {rd }}$ ed., New York / London: W. W. Norton \& Company 
Wallace, N. (1980), The overlapping generations model of fiat money, in: J. Kareken und N. Wallace (eds.), Models of Monetary Economies, Minneapolis: Federal Reserve Bank of Minneapolis, S. 49-82

Weil, P. (1987), Love thy children - Reflections on the Barro debt neutrality theorem, Journal of Monetary Economics, 19, S. 377-391

Weil, P. (1989), Overlapping families of infinitely-lived agents, Journal of Public Economics, 38, S. 183-198

Wellisch, D. (1991), Intertemporale und Internationale Aspekte staatlicher Budgetdefizite, Tübingen: Mohr / Siebeck

Yaari, M. E. (1965), Uncertain lifetime, life insurance, and the theory of the consumer, Review of Economic Studies, 32, S. 137-150

Zilcha, I. (1998), Intergenerational transfers, economic growth and income distribution, in: K.-J. Koch und K. Jaeger (eds.), Trade, Growth, and Economic Policy in Open Economies, Berlin u.a.: Springer, S. 111-121 


\section{Stichwortverzeichnis}

Abschreibungsrate 27, 31, 45f, 205

Absorption 29, 121, 165f

Agent, repräsentativer: s. Akteur, repräsentativer

Akteur, repräsentativer 1, 64

Allokation 71, 74

Angebotseffekt 56

Anlage, reale 77

Anleihe, ewige 60, 87f, 137

Anteilschein 65-67, 69, 75, 77, 82, 88f, 106-108, 112, 119, 175, 179

Arbeitseinkommen 25, 67, 69, 87f, $113,151-154,175,191,200$, $214 \mathrm{f}$

Arbeitskraft 64f, 105

Arbeitskräftepotential 24, 26, 58

Arbitrage 16, 27, 40, 51f, 107

Arbitrageeffekt 56

Arrow-Debreu-Ökonomie 73

Auslandsinvestition 117-119, 127, 167f, 170

Auslandsposition 29, 41, 48-50, 121, 206

Auslandsvermögen 42, 46

Außengeld 12, 75-77

Ausstattungsvektor 12

Autarkie 105-107, 114-116, 123-126, 129f

Autarkiegleichgewicht 106
Blanchard-Modell 5, 187-216

Bodenintensität der Produktion 58

Bruttokomplement 73, 79, 91, 117, $145,177,179,223$

Bruttolohn 40, 43f

Bruttorendite 52, 54-56

Bruttosubstitut 73, 91, 96, 99, 117, $145,155,158,162,166 \mathrm{f}$, 169f, 177, 179, 222

Bubble: s. Preisblase, spekulative Budget, öffentliches $48 \mathrm{f}$

Budgetdefizit

-, primäres $45,49,53,213$

-, staatliches 45, 96, 102-104, $157,160,162-165,171$, 180-183

Budgetrestriktion

-, intertemporale $13,25,39$, $49,67,74,88,197,219$

-, staatliche 40, 45, 48, 53, 87, 94, 213f, 217

cash-in-advance Restriktion 75 Cass-Koopmans-Wachstumsmodell:

s. Ramsey-Wachstumsmodell Clower-Restriktion:

s. cash-in-advance Restriktion Couponzahlung 87, 90, 137, 140 Cournot-Aggregation 143, 145, 147 crowding-out 41 
Deflation 13

Diamond-Modell 4f, 24, 80f, 93f, 104, 120f, 125-127, 134f,

151-153, 164f, 210

Diskontfaktor 25, 66, 69-72, 74, 78f, $82,88-93,107,109-112$, 114-117, 122f, 128, 141-145, 190f, 196, 214f, 224

Diskontierungsfaktor:

s. Diskontfaktor

Dividende 64-66, 77, 87, 106, 113 , 118,140

Dividendenrendite: s. Ertrag

Dynamik 69-71, 111-113, 208-210

Effizienz, dynamische 31, 44, 120f, $125-127,135,152,210$

Einkommenseffekt 72f, 78f, 122f, 146f, 174, 190, 198, 221f

Einkommensexpansionspfad 73, 96f, $155 \mathrm{f}$

Einkommensvektor 63

Engel-Aggregation 143

Erstausstattung 12

Ertrag 66, 85, 101, 132

Erwartungsnutzenmaximierung 189-191

Euler

-Gleichung 64

-Theorem 27, 205

Faktoreinkommen 29, 165

Faktor-Preis-Grenze 34

Finanzkapital 27
Finanzvermögen 29, 192-194, 198-201, 204, 206, 208-212

Freihandel 114-116

Fundamentalwert 66, 77

Gegenwartspreis 67

Geld in der Nutzenfunktion

Annahme 75f

Gewinn 64, 205

Gläubigerland 31f, 119-121, 127, 147f, 151f, 177, 180-183, 211

Gleichgewicht

-, Existenz $72 \mathrm{f}$

-, langfristiges $21,30,72-77$, 89-94, 106, 113-120

-, Optimalität 73-75

-, Stabilität $76 \mathrm{f}$

-, zyklisches 83-86, 174

Grenzrate der Substitution 14, 26, $64,68,88$

Gütermarktgleichgewicht 69,95 , 139, 154

-, temporäres 71

Hameltonfunktion 195

Handelsbilanz 17f, 29, 42, 45-48, $119-121,125,148 \mathrm{f}, 166$, 168-171, 177f, 180, 182f

Heckscher-Ohlin-Außenhandelsstruktur 36, 50, 60, 123

Humanvermögen 191, 198-201, 214-216

Indeterminiertheit 77-81

Inflation 13, 20-22 
Inlandsproduktion 29

Innengeld 20-22, 117-119

Investitionsquote 120

Kapitalakkumulation 54-56

Kapitalertrag $118 \mathrm{f}$

Kapitalertragsteuer 51-57

Kapitalgewinn 66f, 82f, 85, 131, 164, 179

Kapitalgut 26

Kapitalintensität 26, 28, 34, 48, 55f, 125-127, 151, 204, 211

Kapitalmarktgleichgewicht 28, 41, $54,69,89,95,108,138,154$

Kapitalmarktzins 59

s. Zinssatz, langfristiger

Kapitalmobilität, internationale 28, 34-36, 51, 135

Kapitalstock 26, 54, 134, 152, 164

Kapitalverlust 66f, 82f, 85, 99-102, $104,130,159,164,178 f, 180 f$

Konsumfunktion 198

Konsumgut 12, 26

Kurswert 59, 66, 69, 87, 89, 113 , $130-133,144,160,181$

Lagrangefunktion 14, 25, 68

Laissez-Faire-Regime 23, 36, 60, $107,120,122,205$

Lebenserwartung 188

Leistungsbilanz 29, 37-39, 41-43, 46-48, 165-171, 181-183, 206

Lohnsteuer 40

Lucas-Kritik 173
Marktstruktur, kompetitive 26, 64, 107

Nachsteuerrendite: s. Nettorendite Nationalprodukt 26

Nettoauslandsinvestition 30,119 , $165,167,170$

Nettoauslandsvermögen 29, 165 , 206

Nettogrenzprodukt 27

Nettolohn 40, 43f

Nettoneuverschuldung 40

Nettorendite 51f, 54-56

Nominalpreis 13

Normalgut 35, 64, 73, 90f, 96, 99 , $142,146,155,158,162$

Nutzenfunktion

-, indirekte 149-151

-, intertemporale $12,25,63$, 67,105

Nutzenmaximierungsproblem, intertemporales $13,25,67,88$, 105

Offer-Kurve: s. Tauschkurve

Ökonomie, klassische 13, 81

OLG-Modell 1, 4-6, 11

-, mit fixem Produktionsfaktor 57-61

-, mit Kapitalakkumulation 24-56

-, mit Vermächtnis 6f, 218

-, monetäres $12-23,76,78,80$, 86

Optimalität, soziale 31 
Optimierungsmodell 4

Pachtzins 59

Pareto-Optimalität 73

Pauschalsteuer 39, 213, 217

Ponzi-Spiel 194, 213

Portfolio 2, 27, 40f, 87, 104, 140, 175

Portfolio-Autarkie-Regime 22f, 36, 60

Präferenz, offenbarte 92, 123f, 149, 227-229

Präferenzordnung 12, 63

Preisblase, spekulative 66, 75

Preisverhältnis, intertemporales 13 , 16

Produktionsfaktor, fixer 57

Produktionsfunktion, neoklassische 26, 204

Produktionsprozeß 64f

Produktionstechnik 2, 64f, 107, 117, $122,167,170,177$

Produktivkapital 24, 27, $205 f$

Pro-Kopf-Größe 26, 202

Quellenstaatprinzip 51-53, 55-57, $137 \mathrm{f}$

RA-Modell 1, 6f, 173

Ramsey-Wachstumsmodell 4

Reallohn 26f, 58

Realpreis 59, 65, 74

Realtransfer 87

Realzins 24

Regel, goldene 31, 41
Regularität 80f, 174

Rendite 65, 73

Replikation 12, 24, 63, 70

Ricardo-Äquivalenztheorem 6f, 173, 212-218

Roy`s Identität 149f

Samuelson-Ökonomie 13, 20f, 76, $80 \mathrm{f}$

Schuldendienst 40

Schuldentilgung 40

Schuldnerland 31f, 119-121, 127, 147, 151f, 171, 177, 180-183, 211

Schuldschein 118f, 129, 165, 177f

Skalenerträge 64

Slutsky

-Dekomposition 72, 221

-Gleichung 72, 150f

Sozialversicherungssystem $49 \mathrm{f}$

Sparfunktion 69, 88, 106

Sparquote 120

Sparrate 35

Spekulationsblase: s. Preisblase, spekulative

Staatsanleihe 40, 87, 89

Staatskonsum 45

Staatsverschuldung 40, 92-94, 103, 138-142, 175f, 179-183, 217

Stabilität 76, 207

Steady State: s. Gleichgewicht, langfristiges

Sterbewahrscheinlichkeit 187f, 198, 201,216

Steuer 49-51, 55, 87f, 96, 137f 
Struktur, demographische 1, 5, 63, $105,188 \mathrm{f}$

Strukturbruch 81, 128, 174

Substitutionseffekt, intertemporaler 72f, 78f, 122f, 146f, 174, 190, $198,221 \mathrm{f}$

Substitutionselastizität, intertemporale $189 \mathrm{f}$

Tauschkurve 69-73, 83f, 93, 106, 109-112, 223-226

Tauschmittelfunktion 12

Technologie 2,26, 64, 105, 173, 204

Terms of Trade 106, 123, 125,

151-153, 164, 173, 175, 177

Effekt, intertemporaler 44, 149

Territorialprinzip:

s. Quellenstaatprinzip

Transfer 43, 87, 96, 137f, 153, 158, 162f, 175f, 181

Transversalitätsbedingung 195f, 213

Überschußnachfragefunktion 14 , $220 \mathrm{f}$

Umlageverfahren 49f

Umverteilungspolitik, intergenerationale 2

Umverteilungsproze $\beta$, gesellschaftlicher 44

Universalprinzip:

s. Wohnsitzstaatprinzip

Utilitarismus $103 \mathrm{f}$

Vermächtnismotiv 6
Vermögenstitel 65

Versicherung $192 \mathrm{f}$

Verzinsung 66-68, 88, 98, 151, 154

Verzinsungsfaktor 25

Volkseinkommen 29

Voraussicht, vollkommene 13, 24, 59,65

Wachstumsmodell 4f

Walras-Gesetz 14, 18f, 28, 41, 54, $90,109,140,221,224$

Walras-Gleichgewicht 18, 28, 69, 89, 109, 139

Wechselkurs 23

Wechselkursindeterminiertheit 22f

Weil-Modell 6, 216-218

Weltprinzip: s. Wohnsitzstaatprinzip Wert, innerer: s. Fundamentalwert Wertaufbewahrungsfunktion 12 Wohlfahrt 36, 43f, 56f, 92-94, 102-104, 123-127, 133-135, 149-153, 162-165, 176-179, 181,227

Wohnsitzstaatprinzip 51-57, $137 \mathrm{f}$

Zahlungsbilanz 29, 165-171, 181-183

Zeithorizont 1

Zeitpräferenz 37-39, 109, 114, 117, $137,160,173,176,180,191$, 225

Zeitpräferenzrate 3, 33f, 48, 120, 190f, 195, 198, 202f, 208f Zentralbankgeld: s. Außengeld 
Zinsarbitragebedingung 66, 84f, $158 \mathrm{f}$

Zinsdynamik 82f, 85, 99-102, $129-133,158-161,176,180$

Zinsendienst 40, 47f, 87f, 139, 144,

$153,175,194,213,217$

Zinssatz

-, kurzfristiger $59,66,82$,

98-102, 155-161, 173, 175,

$178,180 \mathrm{f}$

-, langfristiger 66f, 98-102,

$157-161,173,176,178,180 f$

Zinsspread: s. Zinsspreizung

Zinsspreizung $82 \mathrm{f}, 86$

Zinsstruktur 67, 83, 85f, 130-133,

$173,176,181$

Zinstheorie, biologische 31

Zinszahlung: s. Couponzahlung 


\section{HOHENHEIMER VOLKSWIRTSCHAFTLICHE SCHRIFTEN}

Band 1 Walter Deffaa: Anonymisierte Befragungen mit zufallsverschlüsselten Antworten. Die Randomized-Response-Technik (RRT). Methodische Grundlagen, Modelle und Anwendungen. 1982.

Band 2 Thomas Michael Baum: Staatsverschuldung und Stabilisierungspolitik in der Demokratie. Zur neoinstitutionalistischen Kritik der keynesianischen Fiskalpolitik. 1982.

Band 3 Klaus Schröter: Die wettbewerbspolitische Behandlung der leitungsgebundenen Energiewirtschaft. Dargestellt am Beispiel der Fernwärmewirtschaft der Bundesrepublik Deutschland. 1986.

Band 4 Hugo Mann: Theorie und Politik der Steuerreform in der Demokratie. 1987.

Band 5 Max Christoph Wewel: Intervallarithmetische Dependenzanalyse in der Ökonometrie. Ein konjekturaler Ansatz. 1987.

Band 6 Heinrich Pascher: Die U.S.-amerikanische Deregulation Policy im Luftverkehrs- und Bankenbereich. 1987.

Band 7 Harald Lob: Die Entwicklung der französischen Wettbewerbspolitik bis zur Verordnung Nr. 86-1243 vom 01. Dezember 1986. Eine exemplarische Untersuchung der Erfassung der Behinderungsstrategie auf der Grundlage des Konzepts eines wirksamen Wettbewerbs. 1988.

Band 8 Ulrich Kirschner: Die Erfassung der Nachfragemacht von Handelsunternehmen. Eine Analyse der ökonomischen Beurteilungskriterien und der wettbewerbsrechtlichen Instrumente im Bereich der Verhaltenskontrolle.1988.

Band 9 Friedhelm Herb: Marktwirtschaftliche Innovationspolitik. 1988.

Band 10 Claus Schnabel: Zur ökonomischen Analyse der Gewerkschaften in der Bundesrepublik Deutschland. Theoretische und empirische Untersuchungen von Mitgliederentwicklung, Verhalten und Einfluß auf wirtschaftliche Größen. 1989.

Band 11 Jan B. Rittaler: Industrial Concentration and the Chicago School of Antitrust Analysis. A Critical Evaluation on the Basis of Effective Competition. 1989.

Band 12 Thomas Märtz: Interessengruppen und Gruppeninteressen in der Demokratie. Zur Theorie des Rent-Seeking. 1990.

Band 13 Andreas Maurer: Statistische Verfahren zur Ermittlung von oligopolistischen Strukturen. 1990.

Band 14 Peter Mendler: Zur ökonomischen und politisch-institutionellen Analyse öffentlicher Kredithilfen. 1992.

Band 15 Heinrich J. Engelke: Die Interpretation der Rundfunkfreiheit des Grundgesetzes: Eine Analyse aus ökonomischer Sicht. 1992.

Band 16 Thomas Fischer: Staat, Recht und Verfassung im Denken von Walter Eucken. Zu den staats- und rechtstheoretischen Grundlagen einer wirtschaftsordnungspolitischen Konzeption. 1993.

Band 17 Stefan Elßer: Innovationswettbewerb. Determinanten und Unternehmensverhalten. 1993.

Band 18 Reinhard Scharff: Regionalpolitik und regionale Entwicklungspotentiale. Eine kritische Analyse. 1993.

Band 19 Karin Beckmann: Probleme der Regionalpolitik im Zuge der Vollendung des Europäischen Binnenmarktes. Eine ökonomische Analyse. 1995. 
Band 20 Bernd Nolte: Engpaßfaktoren der Innovation und Innovationsinfrastruktur. Eine theoretische und empirische Analyse für ländliche Wirtschaftsräume in Baden-Württemberg. 1996.

Band 21 Klaus-Rainer Brintzinger: Die Nationalökonomie an den Universitäten Freiburg, Heidelberg und Tübingen 1918 - 1945. Eine institutionenhistorische, vergleichende Studie der wirtschaftswissenschaftlichen Fakultäten und Abteilungen südwestdeutscher Universitäten. 1996.

Band 22 Steffen Binder: Die Idee der Konsumentensouveränität in der Wettbewerbstheorie. Teleokratische vs. nomokratische Auffassung. 1996.

Band 23 Alexander Burger: Deregulierungspotentiale in der Gesetzlichen Rentenversicherung. Reformnotwendigkeiten versus Reformmöglichkeiten. 1996.

Band 24 Burkhard Scherer: Regionale Entwicklungspolitik. Konzeption einer dezentralisierten und integrierten Regionalpolitik. 1997.

Band 25 Frauke Wolf: Lorenzkurvendisparität. Neuere Entwicklungen, Erweiterungen und Anwendungen. 1997.

Band 26 Hans Pitlik: Politische Ökonomie des Föderalismus. Föderative Kompetenzverteilung im Lichte der konstitutionellen Ökonomik. 1997.

Band 27 Stephan Seiter: Der Beitrag Nicholas Kaldors zur Neuen Wachstumstheorie. Eine vergleichende Studie vor dem Hintergrund der Debatte über den Verdoorn-Zusammenhang. 1997.

Band 28 André Schmidt: Ordnungspolitische Perspektiven der europäischen Integration im Spannungsfeld von Wettbewerbs- und Industriepolitik. 1998.

Band 29 Bernd Blessin: Innovations- und Umweltmanagement in kleinen und mittleren Unternehmen. Eine theoretische und empirische Analyse. 1998.

Band 30 Oliver Letzgus: Die Ökonomie internationalen Umweltschutzes. 1999.

Band 31 Claudia Hafner: Systemwettbewerb versus Harmonisierung in Europa. Am Beispiel des Arbeitsmarktes. 1999.

Band 32 Jürgen Kulle: Ökonomie der Musikindustrie. Eine Analyse der körperlichen und unkörperlichen Musikverwertung mit Hilfe von Tonträgern und Netzen. 1998.

Band 33 Michael Ganske: Intertemporale Aspekte von Staatsverschuldung und Außenhandel. 1999. 